\title{
RESONANT NUCLEAR SCATTERING OF SYNCHROTRON RADIATION: DETECTOR DEVELOPMENT AND SPECULAR SCATTERING FROM A THIN LAYER OF ${ }^{57} \mathrm{Fe}$
}

\author{
Alfred Quentin Rueben Baron \\ Stanford Linear Accelerator Center \\ Stanford Synchrotron Radiation Laboratory \\ Stanford University, Stanford, California 94309
}

SLAC-Report-95-463

April 1995

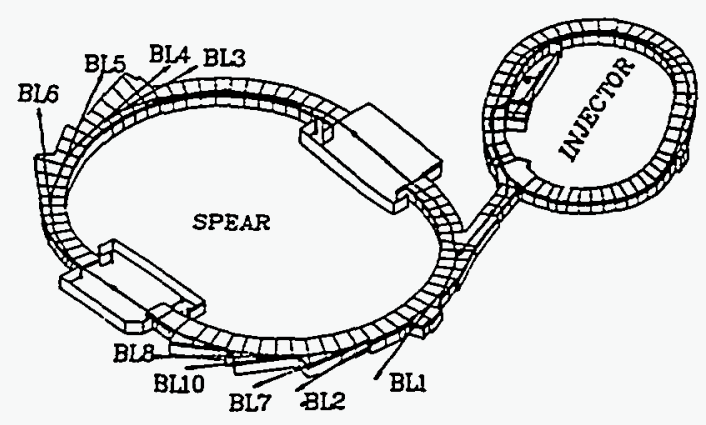

Prepared for the Department of Energy

under contract number DE-AC03-76SF00515

STANFORD LINEAR ACCELERATOR CENTER

STANFORD SYNCHROTRON RADIATION LABORATORY

Stanford University - Stanford, California 
This document and the material and data contained therein, was developed under sponsorship of the United States Government. Neither the United States nor the Department of Energy, nor the Leland Stanford Junior University, nor their employees, nor their respective contractors, subcontractors, or their employees, makes any warranty, express or implied, or assumes any liability or responsibility for accuracy, completeness or usefulness of any information, apparatus, product or process disclosed, or represents that its use will not infringe privately-owned rights. Mention of any product, its manufacturer, or suppliers shall not, nor is it intended to, imply approval, disapproval, or fitness for any particular use. A royalty-free, nonexclusive right to use and disseminate same for any purpose whatsoever, is expressly reserved to the United States and the University. 


\section{DISCLAIMER}

Portions of this document may be illegible in electronic image products. Images are produced from the best available original document. 


\begin{abstract}
This thesis explores resonant nuclear scattering of synchrotron radiation. An introductory chapter describes some useful concepts, such as speedup and coherent enhancement, in the context of some basic physical principles. Methods of producing highly monochromatic synchrotron beams using either electronic or nuclear scattering are also discussed. The body of the thesis concentrates on detector development and specular scattering from synthetic layered materials.
\end{abstract}

A detector employing microchannel plate electron multipliers is shown to have good ( $50 \%$ ) efficiency for detecting $14.4 \mathrm{keV} x$-rays incident at small ( 0.5 degree) grazing angles onto Au or CsI photocathodes. However, being complicated to use, it was replaced with a large area $\left(>=1 \mathrm{~cm}^{2}\right)$ avalanche photodiode (APD) detector. The APD's are simpler to use and have comparable (30-70\%) efficiencies at $14.4 \mathrm{keV}$, subnanosecond time resolution, large dynamic range (usable at rates up to $\sim 10^{8}$ photons/second) and low $(<\sim 0.01 \mathrm{cts} / \mathrm{sec})$ background rates.

Maxwell's equations are used to derive the specular x-ray reflectivity of layered materials with resonant transitions and complex polarization dependencies. The effects of interfacial roughness are treated with some care, and the distorted wave Born approximation (DWBA) used to describe electronic scattering is generalized to the nuclear case. The implications of the theory are discussed in the context of grazing incidence measurements with emphasis on the kinematic and dynamical aspects of the scattering. The theory is shown to simulate the measured specular response of a thin $(240 \AA)$ layer of $57 \mathrm{Fe}$, allowing some additional information about the sample structure to be determined. It is also shown that the integrated delayed counting rate from the sample is largest at the critical angle for total external reflection. This is explained using a DWBA, with interesting implications for allowed, finite order, Bragg reflections. 


\section{Acknowledgments}

There have been many people who have made this thesis possible and here I can, at best, present an incomplete list. Most important are the physicists with whom I have collaborated on resonant nuclear scattering experiments: George Brown, my advisor, whose physical insight and quick grasp of complicated problems has made it a pleasure both to learn from him and to watch him in action; John Arthur, who, in addition to teaching me how to run a beamline and to keeping the Mössbauer group together, has been a good friend and a source of calm clarity in many moments of confusion and doubt; and Stan Ruby, who has provided a steady stream of alternative ideas which has helped keep me on my toes. I have also had the great pleasure of working closely with several physicists from other institutions: Sasha Chumakov who is just plain smart (a genius - ask Lena) and hardworking, as well as modest; Gena Smirnov who has a deep understanding and has often politely pointed out the papers where he measured the neat effect I thought I had just discovered (I also would not want to wrestle with him); and, recently, Uwe van Bürck with whom I hope to continue to collaborate (and eat more noodles!). Finally, my thanks to Dennis Brown, the previous graduate student in the group, whose thesis has been a source of careful clear thought.

SSRL has been a good place to work and I owe a debt of gratitude to almost all of the staff. To name only a few, this includes Sean Brennan, Hal Tompkins, Herman Winick, Heinz-Dieter Nuhn, Alan Winston, Alan Swithenbank, Tom Hostetler, Chuck Troxell, Jr., Jim Sebek, Tom Neal, Peter Boyd, Tracy Yott, Ken Culler, Brian Burdick, Chris Hoover, Glen Kerr, Thomas Nguyen, Katherine Cantwell, Michelle Kearney, Shirley Robinson, Lisa Dunn, and everyone. Thanks guys!

Outside of SSRL, there have been many people who have also helped in this work that I would like to thank: Ed Garwin of SLAC, who allowed me to use his alkali halide evaporation setup and Lee Shere who helped me run it; J.D. Kilkenny and his group (especially Perry Bell) at LLNL for their help with microchannel plates; Ernesto Gramsch (formerly at Advanced Photonix, Inc.) who answered many questions about the API diodes; Paul Webb and Henri 
Dautet of EG\&G for allowing me to use one of the EG\&G APDs and for discussing the results; Eric Gullikson with whom I discussed APD operation.

More personally, I would like to thank many friends, including Gary Woods, fellow graduate student and long time roommate who taught me a little about courtesy in exchange for my sense of humor (and hence may have gotten the short end of the stick); David Eliezer my partner in many endeavors; Chris Donohue, who taught me the ropes; and Lea Ann Jones, may she never lose her sense of humor.

Finally, and most specially, I would like to thank my parents Anise and Steve and the rest of my family, Ken, David, Naomi, Julie, MiAe, Yana, Caryl, and the most recent addition, my wife, Yoko Furuyama. 


\section{Table of Contents}

\section{Introduction}

Background 1

Why Synchrotron Radiation $\quad 2$

Samples Used In Synchrotron Radiation Experiments 3

The Work in This Thesis 5

References 6

2. Introduction to Resonant Nuclear Scattering of Synch. Radiation Introduction $\quad 10$

The Nuclear Cross Section $\quad 10$

An Ideal Absorption experiment in the Frequency Domain 11

A Scattering Viewpoint 14

Fourier Transforms, Causality and the Kramers-Kronig Relation $\quad 15$

The Transmission Experiment in the Time Domain 17

Speedup 19

Enhancement of the Coherent (Radiative) Channel 20

Comment on Information Content of Time Domain Experiments 21

A Note On Signal Rates From Broadened Lines 23

Incoherent Scattering 26

The Broad Bandwidth of Synchrotron Radiation 27

To Build a Better Monochromator 28

Improved Conventional (Electronic Scattering) Monochromators 29

Monochromators Using Nuclear Resonant Scattering 39

References 41

\section{Detectors}

Introduction $\quad 47$

The Plastic Scintillation Coincidence Detector 48

A Microchannel (MCP) Detector 51

Gating the MCP Detector 54

Results With The MCP Detector 56

X-Ray Photocathodes 58

An Avalanche Photodiode (APD) Detector 61 
APD Structure 63

Model for X-Ray Response $\quad 67$

X-Ray Source 69

Electronics 69

X-Ray Efficiency $\quad 72$

Time Response $\quad 73$

Improved Time Response 77

Pulse Height (Charge) Response 78

Detector Dependability $\quad 79$

Recent Results: High Efficiencies and High Rates 81

References 82

4. The Optical Theory for Homogenous Media and Ideal Multilayers

Introduction $\quad 90$

The X-Ray Wave Equation $\quad 90$

Constitutive Relations 92

The Dielectric Tensor in Crystalline and Homogeneous Materials 93

The Dispersion Relation 95

Connection to Quantum Mechanics: The Lorentz Relation 95

Dispersion Relation with a Planar Interface 98

Boundary Conditions at Planar Interfaces $\quad 100$

Isotropic Media 105

Reflectivity from a Resonant Medium 108

Polarization Effects 111

Comparison with GIAR Theory 113

Recursive Solution to the Multilayer Problem 114

Comparison with the Theory of Irkaev, et al. 116

References 117

\section{Rough Interfaces}

Introduction 119

Kinematic Scattering 121

Specular Scattering in the Kinematic Approximation $\quad 123$

Kinematic Scattering by a Plane of Scatterers 124

Kinematic Scattering by an Ideal Interface $\quad 126$

Kinematic Scattering from a Real (Non-Ideal) Interface 127 
Failure of the Kinematic Result at Grazing Incidence 129

Why Bother with a Roughness Correction 132

The Distorted Wave Born Approximation 133

Roughness Correction in a Highly Absorbing Material 134

References 136

6. The Specular Response of a Thin Layer of ${ }^{57} \mathrm{Fe}$

Introduction 138

Sample Preparations and Characterization $\quad 139$

Electronic Reflectivity 139

Auger Analysis $\quad 145$

Conversion Electron Mössbauer Spectrum 147

Oxide Layers and the CEMS Data 150

Time Response Measurements 152

Kinematic vs. Dynamical Characteristics of the Time Response 153

Kinematic Time Response: Quantum Beats 155

Dynamical Effects 161

Slow Beats from the Thin Film Geometry 168

Dynamical Effects on the Quantum Beat Frequency 171

Dynamical Modification of Contrast 173

Comparison With GIAR Films $\quad 174$

Fitting The Time Response $\quad 178$

The Integrated Delayed Reflectivity 186

Comparison With Pure Nuclear Reflections 190

Comparison with Other Mössbauer Techniques 192

Final Comments 195

References 195

$\begin{array}{ll}\text { 7. Concluding Comments } & 201\end{array}$

Appendix A: The Forward Scattering Amplitude

Introduction 204

Multipole Scattering Amplitude 204

Magnetic Dipole Transitions 205

Distribution of Quantization Directions $\quad 209$

References 213 
Appendix B: The Distorted Wave Born Approximation (DWBA) Introduction 214

Terms in the DWBA 214

Scattering Matrix for a Planar Disturbance 218

Simplification for Symmetric Interfaces 219

Result of DWBA 220

Evaluation for Electronic Scattering 220

General Comments 222

References 222 


\section{List of Tables}

1.1 Nuclear transitions in synchrotron radiation experiments 4

2.1 Parameters for some Bragg reflections in crystalline silicon. 32

3.1 History of $1.6 \mathrm{~cm}$ beveled edge diodes. 80

6.1 Count rates and measurement locations for the time response of the iron layer.

153

6.2 Results from fitting the time response of the thin iron layer.

179 


\section{List of Figures}

2.1 Schematic of a Mössbauer transmission experiment. 11

2.2 Results of a frequency domain transmission experiment. 13

2.3 Results of a time domain transmission experiment. 19

2.4 Geometry for an asymmetric Bragg reflection. 30

2.5 Rocking curve for Si (111) reflection. 33

2.6 DuMond diagram for Si (111) reflection. 34

2.7 Dispersive and non-dispersive two crystal geometries. 36

2.8 Experimental setup for high resolution monochromator. 36

2.9 DuMond diagram for high resolution monochromator. 37

2.10 Schematic of the asymmetric nested monochromator. 38

3.1 Schematic of a plastic scintillator coincidence detector. 49

3.2 Schematic of the microchannel plate detector. 52

3.3 Schematic of the vacuum chamber used with the MCP detector. 53

3.4 Effect of a retarding potential on the efficiency of the MCP detector. 55

3.5 Schematic of the gating circuit for the MCP detector. 55

3.6 Efficiency of Au photocathodes. 60

3.7 Efficiency of CsI photocathodes. 61

3.8 Schematic of a beveled edge APD. 64

3.9 Field profile inside the beveled edge APD. 65

3.10 Schematic of a reach through APD. 66

3.11 Field profile of a reach through APD. 66

3.12 Absorption (1/e) length for x-rays in silicon. 67

3.13 Schematic of the electronics with the APD detectors. $\quad 70$

3.14 APD response (scope trace) at $14.4 \mathrm{keV}$. 71

3.15 APD efficiency with energy. $\quad 73$

3.16 APD time response. $\quad 75$

3.17 Variation in APD time response with photon energy. $\quad 76$

3.18 Slow efficiency for beveled edge diode. $\quad 76$

3.19 Improved time resolution with higher discriminator threshold. $\quad 78$

$\begin{array}{ll}3.20 \text { Beveled edge pulse height response. } & 79\end{array}$

3.21 Scope trace at very high rates. 82

4.1 Geometry for dielectric stack. 101 
$\begin{array}{ll}4.2 & \text { Fresnel reflection and transmission. } \\ & 108\end{array}$

4.3 Index of refraction with a resonant transition. 109

4.4 Specular reflectivity with a resonant transition. 111

4.5 Reflectivity with linear eigenpolarizations. 112

4.6 Reflectivity with circular eigenpolarizations. 113

4.7 Wave fields for a recursive solution to the multilayer problem. 115

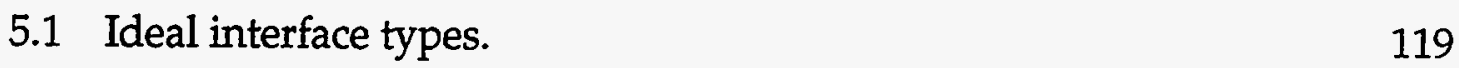

5.2 Specular scattering geometry. 123

5.3 Effect of roughness on the reflectivity. 129

5.4 Fresnel reflectivity with roughness modification. 130

5.5 Kinematic roughness correction vs. graded interface. 131

5.6 Effect of roughness with a resonant transition. 134

5.6 Effect of absorption on the roughness correction. 135

6.1 X-ray reflectivity of the thin iron layer at $14.4 \mathrm{keV} . \quad 140$

6.2 Three layer model of the iron layer. 141

6.3 Many layer models of the iron layer. 142

6.4 Comparison of measure reflectivity with large and small slit. $\quad 145$

6.5 Sputter Auger profile. 146

6.6 Conversion electron Mössbauer spectra. 148

6.7 Fits to the iron layer CEMS. 149

6.8 Locations used to measure the time response. 152

6.9 Time response with a parallel magnetic field. 154

6.10 Time response with a perpendicular magnetic field. 155

6.11 Index of refraction for parallel field eigenpolarizations. 158

6.12 Kinematic time response, parallel field. 159

6.13 Index of refraction for perpendicular field eigenpolarization. 160

6.14 Kinematic time response, perpendicular field. 161

6.15 Single line index of refraction. 163

6.16 Grazing incidence reflectivity for single line transition. 164

6.17 Frequency spectrum of the delayed response. 166

6.18 Centroid shift with angle. 167

6.19 Time response in specular reflection. 168

6.20 Electronic reflectivity profiles with different indices. 169

6.21 Thin layer effect in specular reflection. $\quad 170$

6.22 Effect of dynamical scattering on line separations. 172 
6.23 Effect of dynamical scattering on beat patterns. 173

6.24 Comparison of GIAR and specular frequency response. 176

6.25 Comparison of GIAR and specular time response. 177

6.26 Linear scale fit results to thin iron layer, perpendicular field. 181

6.27 Log scale fit results to thin iron layer, perpendicular field. 182

6.28 Linear scale fit results to thin iron layer, parallel field. 183

6.29 Log scale fit results to thin iron layer, parallel field. 184

6.30 Delayed intensity in specular reflection. $\quad 187$

6.31 Delayed intensity measured from the thin iron layer. 189

6.32 Delayed intensity from an allowed Bragg reflection. 191

6.33 Delayed intensity in specular reflection for several samples. 193

B.1 DWBA correction to the reflectivity 215

B.2 DWBA correction to the transmission 215 


\section{Introduction}

\section{Background}

Resonant nuclear scattering of synchrotron radiation is a field that begins at the interface of two more well established fields. These two fields are resonant nuclear scattering, particularly the Mössbauer effect, and x-ray scattering techniques in general, especially those used to manipulate synchrotron radiation.

Nuclear resonant scattering experiments using radioactive sources date back to the early (if unsuccessful) experiments by Kuhn [Kuhn, 1929, 1] to see increased absorption due to the presence a resonant transition in radium, with successful observation of resonant scattering from ${ }^{198} \mathrm{Hg}$ by Moon [Moon, 1951, 2]. One of the main sources of difficulty in these early experiments was that the recoil of an emitting atom resulted in a sufficient Doppler shift of the radiation so that a photon emitted by the decay from one nucleus would not readily excite another nucleus. However, various methods were devised to circumvent this problem, though not easily (see the review article [Metzger, 1959, 3]).

Mössbauer, [Mössbauer, 1958, 4] discovered that recoil free nuclear transitions were possible in solid materials. This made the observation of resonant scattering and absorption significantly easier, paving the way for many fascinating physics experiments (see reprints in [Frauenfelder, 1962, 5]). In addition, the fact that the nuclear response is sensitive to its local environment, that of the atomic electrons, which is in turn affected by local structure and bonding, opened the field of Mössbauer hyperfine spectroscopy (see, e.g., [Greenwood and Gibb, 1971, 6] [Dickson and Berry, 1986, 7]).

In 1974 it was suggested both by Ruby [Ruby, 1974, 8] and by Mössbauer [Mössbauer, 1974, 9] that one might use synchrotron radiation to excite resonant nuclei instead of the radiation from radioactive sources. In 1985, the first clear signal in a synchrotron radiation nuclear scattering experiment was seen by Gerdau et al., [Gerdau, et al., 1985, 10] This opened up a field that is now rapidly 
expanding and will probably flourish as new beamlines devoted to these studies become operational at third generation synchrotron radiation sources* .

\section{Why Synchrotron Radiation}

There are several very good reasons why it is interesting to use synchrotron radiation to excite a nuclear resonance. The simplest is that synchrotron radiation sources are brighter than radioactive sources, even over the very narrow bandwidth of the nuclear resonance (see [Cohen, 1980, 11]). If an experiment requires collimation $<\sim 10^{-7} \mathrm{sr}$., the count rates with synchrotron radiation will be higher than those using a radioactive source. Thus, for example, nuclear diffraction experiments become much easier using synchrotron radiation. Additionally, the high collimation of synchrotron radiation means that one may make use of conventional (electronic) $x$-ray scattering techniques that employ Bragg reflections (such as polarimetry [Mills, 1991, 12] , and interferometry [Bonse and Graeff, 1977, 13]).

The most interesting facet of synchrotron radiation experiments is that the source is pulsed. Conventional Mössbauer experiments use radioactive sources having line widths comparable to the resonance width, while synchrotron radiation provides broad band impulse excitation. This has many subtle and interesting consequences, some of which are discussed in more detail in chapter

2. However, one useful immediate consequence is that the background from non-resonant scattering processes may be removed by gating in time ([Seppi and Boehm, 1962, 14]). The lifetime of the nuclear resonance is typically much longer than the synchrotron pulse duration, while the time required for non-resonant (electronic) scattering is much shorter. Thus one can remove the background from non-resonant electronic scattering processes and concentrate only on events involving nuclear interaction. In a typical Mössbauer experiment using a radioactive source, one is usually looking for a peak or a dip in a large background, while in a synchrotron radiation experiment, one has essentially no background that is coincident with the data. This, in conjunction with the higher

\footnotetext{
* These include ESRF in France, the APS in the United States and SPring-8 in Japan. Also the undulators on the Accumulator Ring (AR) at KEK in Japan and on PEP in the US (no longer in operation) might be considered in this category as well.
} 
brightness of synchrotron radiation, can reduce measurement times from weeks or months with radioactive sources to minutes or hours with synchrotron radiation.

The properties of synchrotron radiation make it possible to do some experiments that are not possible with radioactive sources. The extremely good signal to background ratio (due to the pulsed source) and the broad band character of the radiation, means that one can in fact observe resonant nuclear scattering without taking advantage of the Mössbauer effect. Thus, one may investigate the scattering from what is sometimes called the "non-resonant fraction" in Mössbauer experiments, allowing the measurement of phonon densities of states (preliminary work in this direction has been done very recently [Seto, et al., 1994, 15] [Chumakov, 1994, 16]). On a more extreme level, one can also look at resonant nuclear scattering from gaseous [Baron, et al., 1994, 17] and liquid samples [Zhang, et al., 1994, 18].

\section{Samples Used In Synchrotron Radiation Experiments}

Initial synchrotron radiation experiments used Bragg reflections in nearly perfect crystals (see [Rüffer, 1992, 19] and references therein). This was largely due to technical reasons: pure nuclear reflections in these crystals were used to prevent the very large quantity of non-resonantly (electronically) scattered photons from overwhelming the detector and preventing detection of nuclear scattering at later times. However, improvements in optics (monochromators) [Faigel, et al., 1987, 20] [Ishikawa, et al., 1992, 21] [Toellner, et al., 1992, 22] and detectors (avalanche photodiodes) [Kishimoto, 1991, 23] [Baron and Ruby, 1993, 24] have allowed the extension of nuclear scattering experiments to many more types of samples.

Coherent nuclear scattering has been observed in forward transmission through thin foils [van Bürck, et al., 1992, 25] and multilayers [Kikuta, et al., 1992, 26]. Nuclear Bragg scattering has also been observed from multilayers, and specular scattering has been observed from thin films [Grote, et al., 1991, 27] [Baron, et al., 1992, 28] and thicker samples [Kikuta, et al., 1992, 26]. Nuclear 
scattering has also been observed from liquid samples [Zhang, et al., 1994, 18] and from gaseous samples [Baron, et al., 1994, 17].

All of the work above (excepting the last) has been performed with the ubiquitous $14.4 \mathrm{keV}$ transition in ${ }^{57} \mathrm{Fe}$. However the number of isotopes which have been used in these experiments is also increasing. Presently, to this author's knowledge, successful experiments have been done with five isotopes, including ${ }^{57} \mathrm{Fe}$ [Gerdau, et al., 1985, 10]. These are $8.4 \mathrm{keV}$ transition in ${ }^{169} \mathrm{Tm}$ [Sturhahn, et al., 1991, 29], the $23.9 \mathrm{keV}$ transition in ${ }^{119} \mathrm{Sn}$ [Alp, et al., 1993, 30, Kikuta, 1993, 31], the $9.4 \mathrm{keV}$ transition in $83 \mathrm{Kr}$ [Johnson, et al., 1994, 32] [Baron, et al., 1994, 17] and, most recently, the $6.2 \mathrm{keV}$ transition in ${ }^{181} \mathrm{Ta}$ [Chumakov, et al., 1994, 33]. Table 1.1 lists relevant properties of the nuclear transitions observed, as well as some other likely candidates for synchrotron radiation studies.

\begin{tabular}{|c|c|c|c|c|c|}
\hline Isotope & $\begin{array}{l}\text { Trans. } \\
\text { Energy } \\
(\mathrm{keV})\end{array}$ & Transition & $\begin{array}{c}\text { Lifetime } \\
\text { (ns) }\end{array}$ & $\begin{array}{c}\text { Nat. } \\
\text { Abundance } \\
(\%)\end{array}$ & Alpha \\
\hline${ }^{181} \mathrm{Ta}$ & $6.216^{(a)}$ & E1 $(9 / 2->7 / 2)$ & $8730(b)$ & 100 & $71(\mathrm{c})$ \\
\hline${ }^{169} \mathrm{Tm}$ & 8.41 & $\operatorname{M1}(3 / 2->1 / 2)$ & 5.8 & 100 & 220 \\
\hline${ }^{83} \mathrm{Kr}$ & $9.404^{(d)}$ & $\operatorname{M1}(9 / 2->7 / 2)$ & 212 & 11.5 & 20 \\
\hline${ }^{57} \mathrm{Fe}$ & $14.413(\mathrm{e})$ & $\mathrm{MI}(3 / 2->1 / 2)$ & 141 & 2.2 & 8.2 \\
\hline${ }^{119} \mathrm{Sn}$ & 23.9 & $\mathrm{M} 1(3 / 2->1 / 2)$ & 25.6 & 8.6 & 5.2 \\
\hline${ }^{73} \mathrm{Ge}$ & 13.3 & E2 $(9 / 2->5 / 2)$ & 4300 & 7.8 & $\sim 1200$ \\
\hline${ }^{151} \mathrm{Eu}$ & 21.6 & $\mathrm{M} 1(7 / 2->5 / 2)$ & 13.7 & 48 & 29 \\
\hline${ }^{149} \mathrm{Sm}$ & 22.5 & $\mathrm{M} 1 \quad(7 / 2->5 / 2)$ & 10.4 & 14 & $\sim 12$ \\
\hline${ }^{161} \mathrm{Dy}$ & 25.6 & M1 $(7 / 2->5 / 2)$ & 40 & 19 & 2.5 \\
\hline
\end{tabular}

Table 1.1. Nuclear transitions of interest for synchrotron radiation experiments. The top portion of the table shows the transitions for which an effect has been observed while the lower portion shows some transitions which have not yet been investgated. The data for the table comes from [Greenwood and Gibb, 1971, 6], [Lederer and Shirley, 1978, 34] and [Shenoy and Wagner, 1978, 35], unless otherwise noted. Other references are (a) $=[$ Chumakov, et al., 1994, 33], (b) =[Mouchel, et al., 1981, 36], (c) =[Firestone, 1991, 37]

[Campbell and Martin, 1976, 38], (e)=[Baron, et al., 1994, 17] $(f)=[$ Bearden, 1965, 39]. 


\section{The Work In This Thesis}

The immediate purpose of a thesis is to prove to a small audience that one has completed sufficient work to merit the degree under consideration. Of course, implicit in this statement is that the work in the thesis be of a certain breadth, depth and quality. However, on a much more personal level, one would like the thesis to be the sort of document that one might have handed to a younger version of one's self and say "here, this answers most of the questions that you might have if you were to begin to study this field in depth." However, these two goals for a thesis do not entirely overlap. The first is incentive to study the minutiae of the field, to do something new, and then be done with it. The second is a incentive to carefully map out one has learned in years needed to complete the degree.

This thesis attempts both to discuss some new work and provide background sufficient that someone not familiar with the field might pick it up and learn a little. Chapter 2 provides a lengthy introduction to many of the ideas that are important in resonant nuclear scattering experiments using synchrotron radiation, contrasting these experiments with more conventional Mössbauer work using a radioactive source. It is worth pointing out that although the ideas in chapter 2 are not revolutionary, and are not difficult to understand when properly approached, they are also not necessarily obvious. Some of the ideas discussed in the chapter have been the subject of more than a little heated discussion between experienced people working in the field.

Chapter 3 discusses the development of detectors for resonant nuclear scattering experiments using synchrotron radiation. In particular, while the physics of these experiments allows essentially complete separation of the nuclear scattering from non-resonant (electronic) backgrounds, there are serious practical difficulties in making a detector that is both efficient and fast enough to do the separation. The detectors developed as part of this work are a significant improvement over those that were in use previously.

The remainder of the thesis concentrates on describing the nuclear response of thin layered materials excited at grazing incidence. This is an area that has been explored previously using radioactive sources, but the time 
response measured with synchrotron radiation in specular reflection has not been studied. Chapter 4 develops theory to describe grazing incidence scattering from multilayer structures, including complex polarization effects and resonant scattering. Chapter 5 discusses the effects of interfacial roughness. Finally, Chapter 6 describes the in depth analysis of the response of a thin layer of ${ }^{57} \mathrm{Fe}$ excited at grazing incidence. The theory of the previous two chapters is applied and shown to simulate the measured results, as well as providing additional information about the nuclear structure of the sample.

\section{References For Chapter 1}

1 W. Kuhn, Scattering of Thorium C" gamma-radiation by Radium G and ordinary lead. Philosophical Magazine 8 (1929) 625.

2 P.B. Moon, Resonant Nuclear Scattering of Gamma Rays: Theory and Preliminary Experiments. Proceedings of the Physical Society of London A76 (1951) 76.

3 F.G. Metzger, Resonance Fluorescence In Nuclei, in Progress In Nuclear Physics, O.R. Firsch, Editor. New York: Pergamon Press (1959) p. 54.

4 R.L. Mössbauer, Kernresonanzfluoreszenz von Gammastrahlung in Ir ${ }^{191}$. Z. Physik 151 (1958) 124.

5 H. Frauenfelder, The Mössbauer Effect. New York: W. A. Benjamin (1962).

6 N.N. Greenwood and T.C. Gibb, Mössbauer Spectroscopy. London: Chapman and Hall, Ltd. (1971).

7 D.P.E. Dickson and F.J. Berry, ed. Mössbauer Spectroscopy. Cambridge: Cambridge University Press (1986).

8 S.L. Ruby, Mössbauer Experiments Without Conventional Sources. J. Phys. (Paris) C 6 (1974) 209.

9 R.L. Mössbauer. . in Proceedings of the International Union of Crystallography. 1974. Madrid: Unpublished p. 463.

10 E. Gerdau, R. Rüffer, H. Winkler, W. Tolksdorf, C.P. Klages, and J.P. Hannon, Nuclear Bragg Diffraction of Synchrotron Radiation in Yttrium Iron Garnet. Phys. Rev. Lett. 54 (1985) 835. 
11 R.L. Cohen, Nuclear resonance experiments using synchrotron radiation sources, in Synchrotron Radiation Research, H. Winick and S. Doniach, Editor. New York: Plenum (1980) p. 647.

12 D.M. Mills, Techniques of production and analyis of polarized synchrotron radiation. Optical Engineering 30 (1991) 1155.

13 U. Bonse and W. Graeff, X-Ray and Neutron Interferometry, , Editor. Berlin: Springer-Verlag (1977).

14 E.J. Seppi and F. Boehm, Nuclear Resonance Excitation Using a Diffraction Monochromator. Phys. Rev. 128 (1962) 2334.

15 M. Seto, Y. Yoda, S. Kikuta, X.W. Zhang, and M. Ando, Observation of Nuclear Resonant Scattering Accompanied by Phonon Excitation using Synchrotron Radiation. (1994) Submitted for Publication.

16 A.I. Chumakov, private communication (1994).

17 A.Q.R. Baron, A.I. Chumakov, S.L. Ruby, J. Arthur, G.S. Brown, G.V. Smirnov, and U. van Bürck, Nuclear Resonant Scattering of Synchrotron Radiation by Gaseous Krypton. (1994) Submitted for Publication.

18 X.W. Zhang, Y. Yoda, M. Seto, M. Ando, and S. Kikuta, Nuclear Excitation of 57Fe Ion in the HCl Liquid by Synchrotron Radiation. (1994) In preparation.

19 R. Rüffer, Nuclear diffraction using synchrotron radiation. Synchrotron Radiation News 5 (1992) 25.

20 G. Faigel, D.P. Siddons, J.B. Hastings, P.E. Haustein, J.R. Grover, J.P. Remeika, and A.S. Cooper, New Approach to the Study of Nuclear Bragg Scattering of Synchrotron Radiation. Phys. Rev. Lett. 58 (1987) 2699.

21 T. Ishikawa, Y. Yoda, K. Izumi, C.K. Suzuki, X.W. Zhang, M. Ando, and S. Kikuta, Construction of a precision diffractometer for nuclear Bragg scattering at the Photon Factory. Rev. Sci. Instrumen. 63 (1992) 1015.

22 T.S. Toellner, T. Mooney, S. Shastri, and E.E. Alp. High energy resolution, high angular acceptance crystal monochromator. in Optics for High-Brightness Synchrotron Beamlines. J. Arthur ed. SPIE Vol 1740, 1992. p. 218.

23 S. Kishimoto, An Avalanche Photodiode Detector for X-Ray Timing Measurements. Nuclear Instruments and Methods in Physics Research A 309 (1991) 603. 
24 A.Q.R. Baron and S.L. Ruby, Time Resolved Detection X-rays Using Large Area Avalanche Photodiodes. Nuclear Instruments And Methods In Physics Research A 343 (1993) 517.

25 U. van Bürck, D.P. Siddons, J.B. Hastings, U. Bergmann, and R. Hollatz, Nuclear Forward Scattering of Synchrotron Radiation. Phys. Rev. B 46 (1992) 6207.

26 S. Kikuta, Y. Yoda, K. Izumi, K. Hirano, N. Horiguchi, T. Ishikawa, X.W. Zhang, H. Sugiyama, M. Ando, M. Seto, C.K. Suzuki, and S. Nasu, Nuclear resonant scattering with an $x$-ray undulator., in X-ray Resonant (Anomalous) Scattering, G. Materlik, C.J. Sparks, andK. Fischer, Editor. Amsterdam: Elsevier (1992) p. 635.

27 M. Grote, R. Röhlsberger, M. Dimer, E. Gerdau, R. Hellmich, R. Hollatz, J. Jäschke, E. Luken, R. Rüffer, H.D. Rüter, W. Sturhahn, E. Witthoff, M. Harsdorff, W. Pfützner, M. Chambers, and J.P. Hannon, Nuclear Resonant Filtering of Synchrotron Radiation by Grazing-Incidence Antireflection Films. Europhys. Lett. 17 (1991) 707.

28 A.Q.R. Baron, J. Arthur, S.L. Ruby, D.E. Brown, A.I. Chumakov, G.V. Smirnov, G.S. Brown, and N.N. Salashchenko, The Time Response of a Thin Film of 57Fe Excited by Synchrotron radiation at Grazing Incidence. Presented at the International Conference on Anomalous Scattering, Malente, Germany (1992).

29 W. Sturhahn, E. Gerdau, R. Hollatz, R. Rüffer, H.D. Rüter, and W. Tolksdorf, Nuclear Bragg Diffraction of Synchrotron Radiation at $8.41 \mathrm{keV}$ Resonance of Thulium. Europhys. Lett. 14 (1991) 821.

30 E.E. Alp, T.M. Mooney, T. Toellner, W. Sturhahn, E. Witthoff, R. Röhlsberger, E. Gerdau, H. Homma, and M. Kentjana, Time Resolved Nuclear Resonant Scattering from ${ }^{119}$ Sn Nuclei using Synchrotron Radiation. Phys. Rev. Lett. 70 (1993) 3351.

31 S. Kikuta, Vancouver, BC: International Conference on Applications of the Mössbauer effect. (1993) .

32 D.E. Johnson, D.P. Siddons, J.Z. Larese, and J.B. Hastings, Observation of nuclear forward-scattering from $83^{\mathrm{Kr}}$ in bulk and monolayer films. Submitted for publication (1994).

33 A.I. Chumakov, A.Q.R. Baron, J. Arthur, S.L. Ruby, G.S. Brown, G.V. Smirnov, U. van Bürck, and G. Wortmann, Nuclear scattering of Synchrotron Radiation by ${ }^{181} \mathrm{Ta}$. In Preparation (1994). 
34 C.M. Lederer and V.S. Shirley, ed. Table of Isotopes. New York: John Wiley and Sons (1978).

35 G.K. Shenoy and F.E. Wagner, ed. Mössbauer Isomer Shifts. Amsterdam: North-Holland Publishing Company (1978).

36 D. Mouchel, A.N. Larsen, and H.H. Hansen, Half-Life of the $6.21 \mathrm{keV}$ Level in ${ }^{181}$ Ta. Zeitschrift für Physik A 300 (1981) 85.

37 R.B. Firestone, Nuclear Data Sheets Update for $A=181^{*}$. Nuclear Data Sheets 62 (1991) 101.

38 J.L. Campbell and B. Martin, Internal Conversion of the $6.2 \mathrm{keV}$ Transition in ${ }^{181}$ Ta. Zeitschrift für Physik A277 (1976) 59.

39 J.A. Bearden, Selection of $W K_{\text {apha1 }}$ as the X-Ray Wavelength Standard. Phys. Rev. 137 (1965) B455. 


\section{Introduction to Resonant Nuclear Scattering of Synchrotron Radiation}

\section{Introduction}

The purpose of this chapter is to provide an introduction to nuclear scattering experiments using synchrotron radiation. This can be divided into two parts: presentation of some of the physics involved and practical details necessary to make experiments work. In order to elucidate the physics, we consider first a classical Mössbauer transmission experiment using a radioactive source and then compare this with a forward scattering (time domain) experiment using synchrotron radiation. This allows some of the basic concepts to be introduced, including that of speedup and coherent enhancement. Also, some of the more subtle differences between time domain and frequency domain experiments are discussed.

Practically, much of the development of the field of resonant nuclear scattering of synchrotron radiation is linked to improvements in x-ray optics (monochromators) and to improvements in detectors. This chapter discusses the optics since they are crucial to much of the following work. Detector development has been a major part of this thesis, and is described in chapter 3 .

\section{Nuclear Cross Sections}

A useful place to begin a discussion of resonant scattering is the cross section of a single resonant nucleus in an atom. For the purposes of this chapter, it is assumed that the excited state is not split into hyperfine components, and the photon polarization is ignored. If a photon of well defined energy, $\hbar \omega$, is incident on the nucleus, then the total cross section for interaction with the nucleus is (see e.g. [Frauenfelder, 1962, 1] p. 7)

\footnotetext{
* Concerning the generality of this form for the cross section, see, e.g. [Perkins, 1987, 2] pp. 124131.
} 


$$
\begin{gathered}
\sigma_{\text {nuc }}(\omega)=\sigma_{\mathrm{n} 0} \frac{\Gamma_{\mathrm{r}}}{\Gamma_{0}} \frac{1}{1+4 \hbar^{2}\left(\omega-\omega_{0}\right)^{2} / \Gamma_{0}{ }^{2}} \\
\sigma_{\mathrm{n} 0}=\frac{2 \mathrm{j}_{\mathrm{e}}+1}{2 \mathrm{j}_{\mathrm{g}}+1} \frac{\lambda^{2}}{2 \pi}
\end{gathered}
$$

where $\omega_{0}$ is the resonance energy and $\Gamma_{0}$ is the natural line width, related to the (1/e) decay time of the excited state by $\Gamma_{0} \tau_{0}=\hbar . \mathrm{j}_{\mathrm{e}}$ and $\mathrm{jg}$ are the excited and ground state nuclear spins and $\lambda$ is the wavelength of the radiation. $\Gamma_{\mathrm{r}}$ is the radiative line width for the transition, and the ratio $\Gamma_{\mathrm{r}} / \Gamma_{0}$ is the probability that an excited nucleus will decay by emitting a photon. Another probable mode of decay for the nucleus is through direct interaction with the atomic electrons, or internal conversion. Internal conversion is the dominant process in most cases and one writes $\Gamma_{\mathrm{r}} / \Gamma_{0}=1 /(1+\alpha)$ where, for the $14.4 \mathrm{keV}$ transition in ${ }^{57} \mathrm{Fe}, \alpha=8.2$. Thus, an excited nucleus in an iron atom decays by emitting a photon only about $11 \%$ of the time; it usually ejects an atomic electron.

\section{An Ideal Absorption Experiment in the Frequency Domain}

With the discovery of the Mössbauer effect, it became possible to (almost) ignore issue of nuclear recoil and perform an extremely simple absorption experiment. Figure 2.1 shows a schematic of such an experiment.

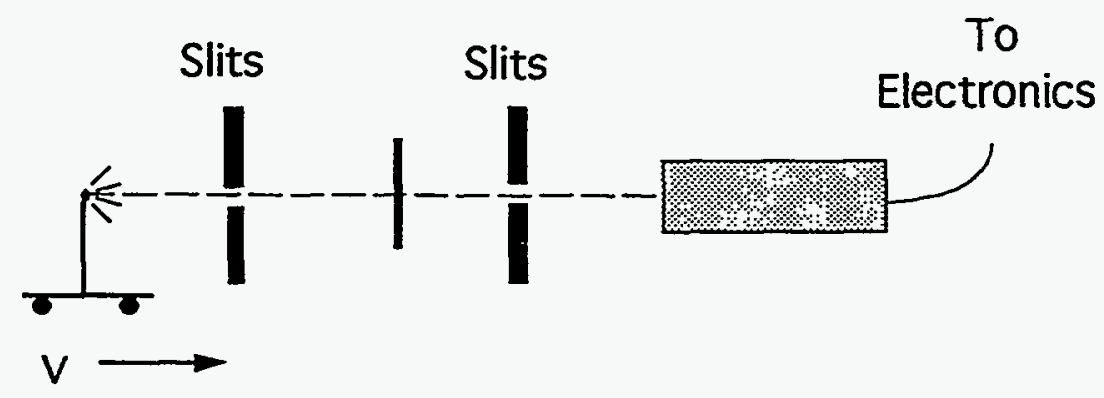

\section{Source Absorber Detector}

Figure 2.1. Schematic of a simple Mössbauer absorption experiment. 
A radioactive source is mounted on a drive that allows one to vary its velocity relative to that of an absorber, assumed at rest. The velocity change results in Doppler shift in the frequency of the photons emitted by the source

$$
\frac{\omega_{v}}{\omega_{v=0}}=\left[\frac{1+v / c}{1-v / c}\right]^{1 / 2} \approx 1+\frac{v}{c}
$$

where $\mathrm{c}$ is the velocity of light and the second expression is valid to lowest order in $\mathrm{v} / \mathrm{c}$ (a very good approximation as $\mathrm{v} \sim 1 \mathrm{~cm} / \mathrm{sec}$ ). Neglecting the source line width, this arrangement provides an ideal tunable source of monochromatic radiation *

The transmission $\mathrm{I} / \mathrm{I}_{0}$, through the absorber, can be measured as a function of velocity of the source, or, equivalently, frequency of the incident radiation. Assuming the absorber is thin, the probability of a photon from the source being absorbed is directly proportional to the cross section, so the transmitted intensity as a function of frequency the source velocity is just (neglecting electronic absorption)

$$
\begin{aligned}
I\left(\omega_{\mathrm{v}}\right) & =I_{0}\left(\omega_{\mathrm{v}}\right)\left(1-N \sigma_{\text {nuc }}\left(\omega_{\mathrm{v}}\right) \mathrm{f}_{\mathrm{r}} \mathrm{L}\right) \\
& =\mathrm{I}_{0}\left(\omega_{\mathrm{v}}\right)\left(1-\frac{\Gamma_{\mathrm{r}}}{\Gamma_{0}} \frac{\mathrm{N} \sigma_{\mathrm{n} 0} L f_{\mathrm{r}}}{1+4 \hbar^{2}\left(\omega_{\mathrm{v}}-\omega_{0}\right)^{2} / \Gamma_{0}^{2}}\right)
\end{aligned}
$$

$\mathrm{N}$ is the number density of resonant nuclei, $\mathrm{L}$ is the thickness of the absorber and $I_{0}$ is the intensity or flux (photons/second) from the source. The quantity $f_{r}$ $\left(f_{r}<1\right)$ is the recoil free fraction of the absorber, sometimes called the LambMössbauer factor. It accounts for the motion of the nuclei in the crystal and, for low energy nuclear transitions one may have $\mathrm{f}_{\mathrm{r}}>\sim 0.5$ at room temperature.

Equation (2.4) is only exact in the limit $L->0$, and more generally one needs to take into account the effect of attenuation of the beam in the first part of the absorber when it interacts with the latter part. Conceptually, one can just

\footnotetext{
* One can not, in practice, neglect the source width, and one of the useful properties of synchrotron experiments is a nearly ideal (impulse) source is provided.
} 
divide the sample up into many pieces to be considered in succession. The more general form, valid for finite $L$, is then

$$
\begin{aligned}
I(\omega) & =\lim _{I \rightarrow \infty} I_{0}(\omega)\left(1-N f_{r} \sigma_{\text {nuc }}(\omega) \frac{L}{I}\right)^{I} \\
& =I_{0}(\omega) \mathrm{e}^{-N f_{\mathrm{r}} \sigma_{\text {nuc }}(\omega) \mathrm{L}}
\end{aligned}
$$

where the v subscript on the frequency has been dropped. Using (2.1) for the cross section, one has

$$
\begin{aligned}
& \frac{I(\omega)}{I_{0}(\omega)}=\exp \left[\frac{-\beta}{1+4 \hbar^{2}\left(\omega-\omega_{0}\right)^{2} / \Gamma_{0}^{2}}\right] \\
& \beta=\sigma_{\mathrm{n} 0} \frac{\Gamma_{\mathrm{r}}}{\Gamma_{0}} \mathrm{NL}=\frac{\lambda^{2} \mathrm{NL}}{2 \pi} \frac{2 \mathrm{j}_{\mathrm{e}}+1}{2 \mathrm{j}_{\mathrm{g}}+1} \frac{\Gamma_{\mathrm{r}}}{\Gamma_{0}}
\end{aligned}
$$

The quantity, $\beta$, is just the number of absorption lengths of the sample exactly at the resonance (neglecting electronic absorption). The transmission, $\mathrm{I} / \mathrm{I}_{0}$, is plotted in figure 2.2 for several different thickness of sample (alpha=8.23).

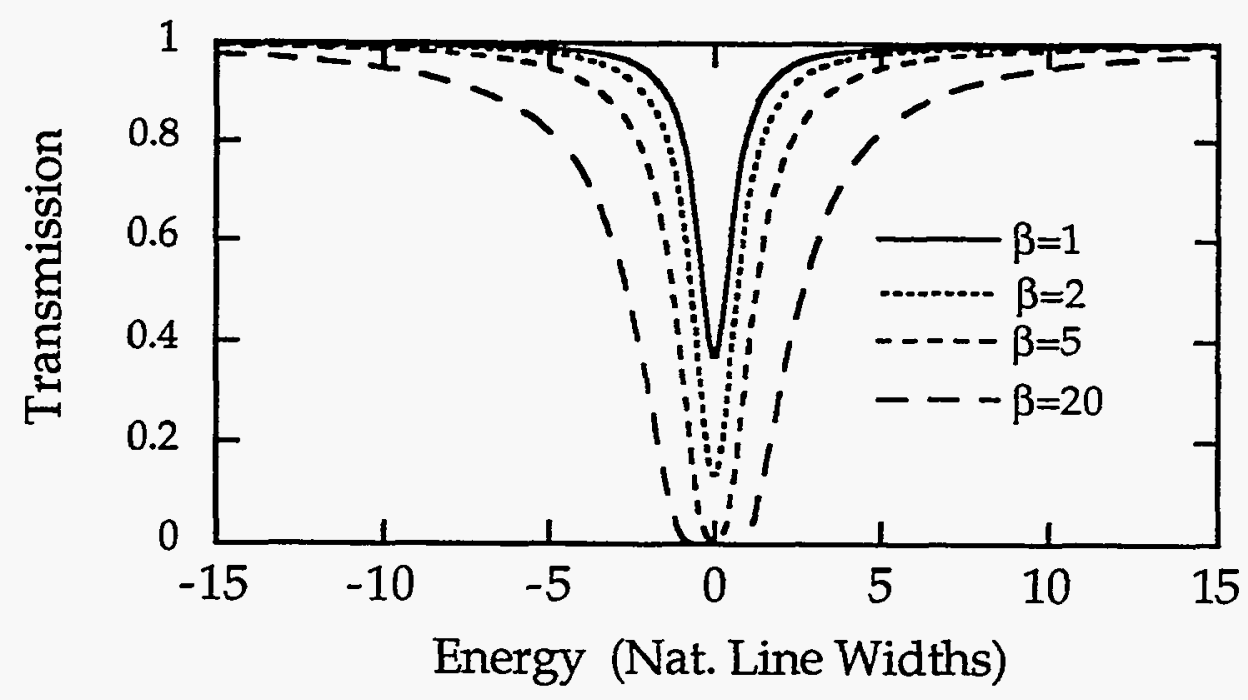

Figure 2.2. Transmission Mössbauer experiment using an ideal source and neglecting electronic absorption. The horizontal axis corresponds to the Doppler shift of the incident photons. 
Note that as the thickness increases, the response saturates, so the measured width of the absorption line increases, becoming significantly larger than the one natural line width appropriate for a thin sample limit.

\section{A Scattering Viewpoint}

It is useful to interpret the results above in terms of a scattering experiment. In particular, instead of considering the probability that a photon is transmitted, $\mathrm{I} / \mathrm{I}_{0}$, one introduces an amplitude whose square is the probability. Formally, in quantum mechanics, one would use $S$ or T-matrix elements.

However, for the purposes of this discussion (and in keeping with the usual language used to describe $x$-ray scattering) we adopt a semi-classical picture, and introduce the electric field amplitude. The transmission of the wave through the absorber can then be described in terms of a complex index of refraction. If the incident wave has amplitude $A_{0}(\omega)$, then the transmitted (or forward scattered) wave will have amplitude

$$
A(\omega)=A_{0}(\omega) e^{+i k \pi(\omega) L}
$$

Where $k=2 \pi / \lambda$ is the wave vector. The index of refraction, $n(\omega)$, may be related to the forward scattering amplitude, F, through the Lorentz relation[Lax, 1951, 3] giving

$$
n(\omega)=1+\frac{2 \pi}{k^{2}} N f_{r} F(\omega)
$$

We have ignored the possible direction (k) dependence of the forward scattering amplitude (assumed a spherically symmetric scatterer). Of course, one measures not the amplitude, but the intensity, so that one has

$$
I(\omega)=|A(\omega)|^{2}=I_{0}(\omega) \exp [-2 \operatorname{Im}\{k n(\omega) L\}]
$$

Equating this with (2.5) gives the optical theorem 


$$
\sigma_{\mathrm{tot}}(\omega)=\frac{4 \pi}{\mathrm{k}} \operatorname{Im}\{\mathrm{F}(\omega)\}
$$

\section{Fourier Transforms, Causality and the Kramers-Kronig Relationship}

All of the systems considered in this thesis are linear and time invariant. Therefore, given the frequency response of the system, one may calculate the impulse or time response through a Fourier transform. If $R(\omega)$ is the frequency response of the system, and $G(t)$ is the impulse response (both complex), one has the relationships

$$
\begin{aligned}
& G(t)=\frac{1}{2 \pi} \int_{-\infty}^{+\infty} R(\omega) e^{-i \omega t} d \omega \\
& R(\omega)=\int_{-\infty}^{+\infty} G(t) e^{+i \omega t} d t
\end{aligned}
$$

A simple, useful, example is the transform pair for a complex Lorentzian:

$$
R(\omega)=\frac{-1}{2 \hbar\left(\omega-\omega_{0}\right) / \Gamma_{0}+i} \Leftrightarrow G(t)=\frac{i}{2 \tau_{0}} e^{-i \omega_{0} t} e^{-t / 2 \tau_{0}} \Theta(t)
$$

$\Theta(t)$ is the Heaviside step function $(\Theta(t<0)=0, \Theta(t>0)=1)$.

We also assume the systems are causal, which is just the time domain statement that there should be no change in the output of a system before the input is changed. The following discussion shows how this time domain statement may be converted to a frequency domain Kramers-Kronig relationship. The derivation is essentially that presented in [Hutchings, et al., 1992,4] while a more conventional derivation may be found in, e.g., [Weissbluth, 1978, 5], p 309. The version below has the advantage of clearly showing the logical relationship between the time and frequency domain statements, but it obscures some of the requirements on $R$, namely that $|R(\omega)|->0$ faster than $1 /|\omega|$ as $\omega \rightarrow$ infinity and that $R$ have no poles in the upper complex plane. 
Mathematically, causality amounts to taking

$$
G(t)=G(t) \Theta(t)
$$

Fourier transforming (2.14) to the frequency domain, one has that

$$
R(\omega)=\frac{1}{2 \pi}(R \otimes \tilde{\Theta})(\omega)
$$

Where the " $\otimes$ " indicates convolution and $\tilde{\Theta}(\omega)$ is the Fourier transform of $\Theta(t)$,

$$
\tilde{\Theta}(\omega)=\mathrm{i} \frac{1}{\omega+\mathrm{i} \varepsilon}=\mathrm{iP} \frac{1}{\omega}+\pi \delta(\omega)
$$

Here $\varepsilon$ is assumed, in the usual way, to be a positive infinitesimal quantity that will be taken to zero after completion of all integrals; P indicates the Cauchy principal value; and $\delta(\omega)$ is the Dirac delta function (see [Heitler, 1954, 6] pp. 6970 and [Merzbacher, 1970,7] p. 85). Evaluation of the convolution and collection of terms then gives the Kramers-Kronig relationship

$$
R(\omega)=\frac{1}{i \pi} P \int_{-\infty}^{+\infty} \frac{R\left(\omega^{\prime}\right)}{\omega^{\prime}-\omega} d \omega^{\prime}
$$

The $i$ in the denominator allows the real part of $R$ to be expressed as an integral over frequency of the imaginary part, and vice-versa. Determining either the real or imaginary part of a causal function is seen to be equivalent to knowing the whole function ${ }^{+}$.

In particular, one requires that the response of a single nucleus, given by $F(\omega)$, be causal. Taking $R(\omega)=F(\omega)$ in (2.17) the optical theorem (2.11) relates the imaginary part of $F$ to the cross section. Then using the form of the cross section, equation 2.1, one finds the total forward scattering amplitude (see also appendix A) is given by

+ It is worth mentioning that measurement of the magnitude of a causal function (e.g. $|R(\omega)|)$ is not sufficient to fully determine the real and imaginary parts of $R$ uniquely, without additional information. The form of $R$ is only determined up to a Blaschke product of additional poles, see [Toll, 1956, 8]. 


$$
\mathrm{F}(\omega)=-\sigma_{\mathrm{n} 0} \frac{\mathrm{k}}{4 \pi} \frac{\Gamma_{\mathrm{r}}}{\Gamma_{0}} \frac{1}{2 \hbar\left(\omega-\omega_{0}\right) / \Gamma_{0}+\mathrm{i}}
$$

\section{The Transmission Experiment in the Time Domain}

Now that we have a form for the scattering amplitude, we return to the transmission or absorption experiment discussed above and consider what the impulse response would be. We take

$$
R(\omega)=\frac{A(\omega)}{A_{0}(\omega)}=e^{+i k n(\omega) L}
$$

Then using the form of the scattering amplitude (2.18) and the Lorentz relation (2.9) one has

$$
\mathrm{kn}(\omega) \mathrm{L}=\mathrm{kL}-\frac{\beta / 2}{2 \hbar\left(\omega-\omega_{0}\right) / \Gamma_{0}+\mathrm{i}}+\frac{2 \pi}{\mathrm{k}} \mathrm{NLF}_{\mathrm{e}}
$$

The third term of (2.20) is just the explicit inclusion of the electronic scattering amplitude. This is frequently put in units of electrons by factoring out the classical radius of the electron, $r_{e}\left(r_{e}=e^{2} / m c^{2}=2.818 \times 10^{-5} \AA\right)$, giving $F_{e}=-r_{e} f_{e}$. The time response is

$$
G(t)=\frac{C}{2 \pi} \int_{-\infty}^{+\infty} \exp \left[\frac{i \beta / 2}{2 \hbar\left(\omega-\omega_{0}\right) / \Gamma_{0}+i}\right] e^{-i \omega t} d \omega
$$

All of the frequency independent parts have been collected in C. This integral was originally done by expanding the exponential and integrating term by term in the complex plane[Kagan, et al., 1979, 9]. However, it is more convenient to make use of the generating function for Bessel functions (see [Abramowitz and Stegun, 1979,10 ] p. 361, equation 9.1.41) which can be written ${ }^{*}$

\footnotetext{
* This approach was first pointed out to me by G. V. Smirnov.
} 


$$
\exp \left[-i \frac{b}{z}-i z t\right]=\sum_{m=-\infty}^{+\infty}\left(\frac{t}{b}\right)^{m / 2}(-i)^{m} z^{m} J_{m}(2 \sqrt{b t})
$$

where $J_{m}$ is the Bessel function (of the first kind) of integer order $m$. The expansion (2.22) is also only valid for $|b|,|t|,|z|>0$. One closes the contour down for $\mathrm{t}>0$ in (2.21) and takes $\mathrm{b}=\beta \Gamma_{0} / 4 \hbar, \mathrm{z}=\omega-\omega_{0}+\mathrm{i} \Gamma_{0} / 2 \hbar$. The only term that survives on integration is the $m=-1$ term, giving a residue of -1 . Then, noting the $t=0$ term gives a delta function and $\mathrm{J}_{-1}(\mathrm{x})=-\mathrm{J}_{1}(\mathrm{x})$, one has

$$
G(t)=C\left[\delta(t)-e^{-i \omega_{0} t} e^{-t / 2 \tau_{0}} \frac{\beta}{4 \tau_{0}} \frac{J_{1}\left(2 \sqrt{\beta t / 4 \tau_{0}}\right)}{\sqrt{\beta t / 4 \tau_{0}}} \Theta(t)\right]
$$

where $\tau_{0}$ is just the natural lifetime, $\tau_{0}=\hbar / \Gamma_{0}$. The step function appears because the integral (2.21) vanishes for $t<0$, since all the pole structure is in the lower half of the imaginary plane. A time domain measurement gives the intensity or

$$
|G(t>0)|^{2}=|C|^{2} e^{-t / \tau_{0}}\left[\frac{\beta}{4 \tau_{0}}\right]^{2}\left[\frac{J_{1}\left(2 \sqrt{\beta t / 4 \tau_{0}}\right)}{\sqrt{\beta t / 4 \tau_{0}}}\right]^{2}
$$

The quantity $\mathrm{ICl} 2$ is just the electronic transmission of the sample. Note the first zero of $J_{1}(x)$ occurs at $x=3.83$ or the first zero of the time response will be at $t=14.7$ $\tau_{0} / \beta$. The time response is plotted for several values of $\beta$ in figure 2.3 , with $\tau_{0}=$ $140 \mathrm{~ns}$. Note the increase in decay rate as the thickness $(\beta)$ increases, and the appearance of the first zero at large thicknesses. 


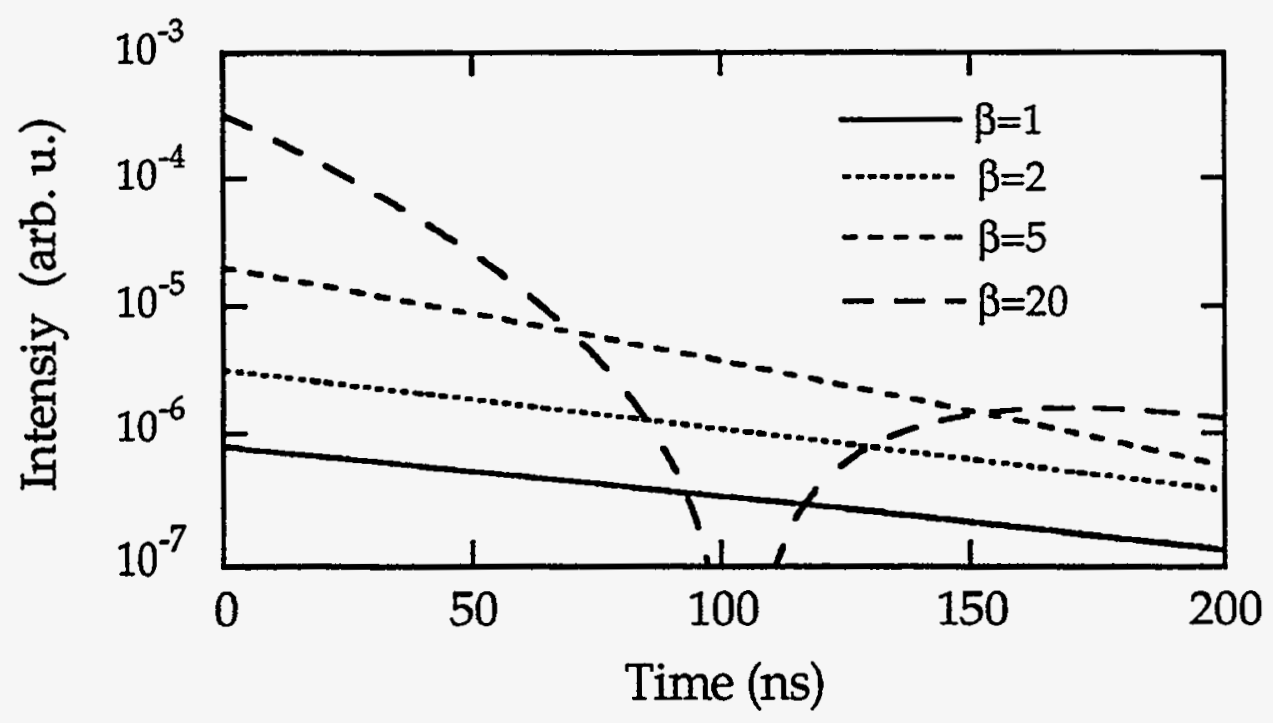

Figure 2.3. Nuclear response in forward scattering for absorbers of various thicknesses after pulse excitation at $\mathrm{t}=0$.

\section{Speedup}

Figure 2.3 provides an example of a general phenomenon common to many time-domain nuclear scattering experiments. In particular, as the number of nuclei in a sample that are excited in phase increases, the coherent time response of the sample becomes faster or speeds up. Thus, as one makes a sample thicker in a forward scattering geometry [van Bürck, et al., 1992, 11], or approaches the exact (index corrected) Bragg angle in a pure nuclear reflection [Smirnov, et al., 1984, 12] [Rüffer, et al., 1987, 13] [van Bürck, et al., 1987, 14], or as one approaches the critical angle in grazing incidence reflection[Baron, et al., $1992,15]$, one finds that the decay time of the sample, excited as a whole, is shorter than the natural decay rate.

The change in the lifetime is a multiple scattering effect. It may be traced all the way back to a simple system of two oscillators discussed by Trammel [Trammell, 1961, 16]. In the case above, it is easily seen that in the thin sample limit, where multiple scattering is ignored (e.g. equation. 2.4, or the small $\beta$ limit of 2.18 or 2.20 ), there will be no change in the lifetime. More generally, one notes that within a Born approximation limit (where multiple scattering is ignored, and 
all nuclei see the same incident wave) the scattering amplitude appropriate for scattering from a single nucleus is just replaced by the phased sum of the scattering amplitudes from all the scatterers:

$$
F_{B A}\left(\hat{\mathbf{k}}, \hat{\mathbf{k}}^{\prime}, \omega\right)=\sum_{1} \mathrm{e}^{\mathrm{i}\left(\mathbf{k}^{\prime}-\mathbf{k}\right) \cdot \mathbf{r}_{1}} F_{1}\left(\hat{\mathbf{k}}, \hat{\mathbf{k}}^{\prime}, \omega\right)
$$

Here $F_{1}\left(\hat{\mathbf{k}}, \hat{\mathbf{k}}^{\prime}, \omega\right)$ is just the scattering amplitude for scattering of an incident photon of frequency $\omega$ and direction $\hat{\mathbf{k}}$ into direction $\hat{\mathbf{k}}^{\prime}$. Inelastic scattering and lattice (phonon) effects are ignored. The sum is over all nuclei in the sample which are fixed at the locations $\mathbf{r}_{\ell}$. Assuming that all nuclei in the sample are equivalent, one can factor $F_{1}$ out of the summation. Thus, in a Born approximation or single scattering limit, the frequency dependence of the total scattering amplitude is just that of a single nucleus, and, consequently, the time response, up to a geometry dependent scale factor, is also that of a single nucleus.

One notes that there is a change in the cross section, the square of the scattering amplitude, which is dependent on the geometry in the Born approximation limit. This is a result of the coherence of the scattering from the individual nuclei and leads to an increased probability of scattering a photon out of the incident beam.

Finally, it is worth noting that the dynamical limit is the rule, rather than the exception, for nuclear scattering with highly isotopically enriched samples: $1000 \AA$ of pure material can be enough to scatter a significant fraction of the incident beam $(\beta>1)$. Therefore, speedup is commonly observed in nuclear scattering experiments.

\section{Enhancement of the Coherent (Radiative) Channel}

In the context of the discussion immediately above, it is good to stress one of the important consequences of exciting a collection of nuclei using radiation: the probability of coherently scattering a photon may be enhanced, relative to the probability to that of an incoherent event (e.g. internal conversion). At the semiclassical level, this just results from the coherent phased addition of the waves 
scattered by the individual nuclei. Thus, when one carries out the sum in (2.25) and then squares to get a cross section, the result can scale faster than simply the number of scatterers*. However, the incoherent processes, such as internal conversion, do not add with well defined phases and hence one squares before summing, so the incoherent cross section scales only linearly with the number of scatterers. Thus the coherent radiative channel is "enhanced" relative to incoherent channels, such as internal conversion.

On a quantum mechanical level the enhancement has to do with the fact that, for a coherent scattering event, it is not possible to determine which nucleus did the scattering, while for incoherent events there is a mark in the sample (e.g. an electron from internal conversion). This is nicely described in the paper [Hannon and Trammell, 1989, 18], though the work in the paper relies on previous work [Trammell and Hannon, 1978, 19] [Trammell and Hannon, 1979, 20] [Trammell, 1961,16] and is also similar to work by other authors [Kagan and Afanas'ev, 1972, 21].

Finally, we note that the discussion based on equation (2.25) is a kinematic one. In dynamical scattering, the enhancement of the radiative channel corresponds to a broadening in the width of the collective response (i.e. the FWHM of the frequency response becomes larger). Thus, since most nuclear scattering experiments are dynamical, enhancement is often associated with a broadening of the frequency response (e.g. in dynamical Bragg diffraction from electronically forbidden reflections [van Bürck, et al., 1980, 22]) and sometimes considered to be the frequency domain analog of speedup.

\section{Comment on Information Content of Time Domain Experiments.}

It is interesting to consider the information content of a time domain forward scattering experiment. Here we do this on a rather abstract level and conclude that if one wishes to measure the forward scattering amplitude of a sample (as in Mössbauer spectroscopy), then, very generally, one is better off with the results of the (idealized) frequency domain experiment than with the

\footnotetext{
"See [Trammell and Hannon, 1988, 17].
} 
results of a time domain measurement. However, it is important to emphasize that, on a practical level, one can usually get as much information from the time domain as the frequency domain. Furthermore, using synchrotron radiation, the results may both have better statistics and require shorter measurement times than when radioactive sources are used. This, in conjunction with the high polarization and collimation of synchrotron radiation, make time domain experiments using synchrotron radiation extremely attractive.

Knowing the absorption as a function of frequency allows direct determination of the imaginary part of the scattering amplitude. A KramersKronig transformation then determines the real part. If the experimental goal is to measure the forward scattering amplitude of a sample, the best a time domain experiment can hope to do is equal the (ideal) frequency domain experiment. However, on very general level, the time domain experiment contains less information than the frequency domain experiment because one does not measure the impulse response, $G(t)$, but its square, $|G(t)|^{2}$. Unlike the case for $|R(\omega)|^{2}$, there is no convenient relationship between $|G(t)|^{2}$ and a causal function. Thus, for the simple case of a Lorentzian response with a thin sample (equation 2.13) or even a thick sample (equation 2.24), the time domain experiment is only sensitive to the line width, and not its location $\left(\omega_{0}\right)$, while the frequency measurement is sensitive to both.

On an intuitive level one might expect that, with the exception of not providing an absolute frequency standard, the time domain experiments should have essentially the same information as the frequency domain experiments. If for example, the absorber response consisted of several lines, one would expect to be able to determine their widths, amplitudes and relative positions from the beat pattern observed in a time domain experiment. Certainly, in the cases this author is familiar with, this has been true. However, this is largely due to substantial a-priori information about the sample. Ideally, one would like a way of inverting, at least theoretically (if not when one includes experimental errors), the measured time response, $|G(t)|^{2}$ to provide either $R(\omega)$ or $F(\omega)$, up to a frequency offset. However, this author has not been able to do so, nor found references in which the problem is addressed. 
Finally, it is worth noting that a time domain experiment can allow one to specifically focus on physical quantities of interest, when they may be obscured in a frequency domain experiment. This is the case when the sample studied may be vibrating. The frequency response of a vibrating sample (or source) will have sidebands due to phase modulation [Ruby and Bolef, 1960, 23]. However, if the vibrations of the sample have a period long compared to the synchrotron pulse then the phase modulation of the nuclear scattered radiation due to the vibrations will not effect the shape of the temporal intensity distribution [Shvyd'ko, et al., 1993, 24]. This is the result of the insensitivity of the time response to the absolute frequency of the resonance. As long as the excitation occurs in a period short compared to the vibration period and the motion of the sample is uniform over the coherently responding volume of the sample (e.g. the product of the extinction length or thickness and the Fresnel zone size), the vibrations only modify phase of the time response, and do not affect the intensity measurement $t^{+}$. Thus there is the possibility to study the time domain effects of transitions between nuclear sublevels that are externally induced by external if fields without the blurring effects that can appear due to the vibration of the sample [Shvyd'ko, et al., 1994, 26].

\section{A Note on Signal Rates From Broadened Lines}

One of the interesting differences between a frequency domain absorption experiment and a time domain forward scattering experiment is in the effect of broadening of the Mössbauer line*. Until this point, we have assumed that all nuclei in a sample are identical, and, in the limit of a thin scatterer, one would observe the natural line width in a frequency domain absorption experiment, or the natural lifetime in the time domain experiment. However, practically, it is often the case that there are shifts in the centers of the lines from nucleus to nucleus (due to differences in the atomic environments), leading to an effective broadening of the width observed in a transmission experiment.

\footnotetext{
+ Strictly speaking, this is only true if there is only one sample in the beam. Addition of another resonant sample will lead to the appearance of interesting interference or echo effects in the measured time response[van Bürck, et al., 1994, 25].

* The author would like to thank A.I. Chumakov for clearly pointing this out.
} 
For conventional absorption experiments using a radioactive source, one would expect, for a thin sample, that the total (frequency integrated) absorption should be the same, broadened or not broadened. Furthermore, for thick samples, with saturation, the integrated absorption for a broadened line will be larger than that for an un-broadened line with the same number of nuclei. Thus, up to the point where the broadening prevents the signal from being seen above background, broadening does not reduce the integrated signal, and can even increase it, in an absorption experiment. However, for a time domain impulse response measurement in forward scattering, it turns out exactly the opposite is true: the broadening reduces the signal.

In many cases, the distribution of nuclear transition energies can be approximated by a Lorentzian, which we take to have width $W \Gamma_{0}(\mathrm{~W}$ dimensionless) and central frequency $\tilde{\omega}$. The probability of a nucleus having a transition frequency in the range $d \omega_{0}$ about $\omega_{0}$ is just

$$
\mathrm{D}\left(\omega_{0}\right) \mathrm{d} \omega_{0}=\frac{2 \hbar}{\pi W \Gamma_{0}} \frac{1}{4 \hbar^{2}\left(\omega_{0}-\tilde{\omega}\right)^{2} /\left(W \Gamma_{0}\right)^{2}+1} \mathrm{~d} \omega_{0}
$$

Note that $\mathrm{D}$ is normalized so the integral of $\mathrm{D}$ over frequency is one. One then must average the scattering amplitude or cross section over this distribution. Performing the integral, one finds that the effect can be included in the scattering amplitude (2.15) or the cross section (2.1) by just increasing the line width of the transition and taking the central frequency to be that of the distribution. One takes

$$
\begin{aligned}
& \Gamma_{0} \rightarrow \Gamma=\Gamma_{0}(1+W) \\
& \tau_{0} \rightarrow \tau=\tau_{0} /(1+W) \\
& \beta \rightarrow \beta^{W}=\beta /(1+W)
\end{aligned}
$$

Neglecting the distinction between $\tilde{\omega}$ and $\omega_{0}$, the scattering amplitude becomes 


$$
\begin{aligned}
\mathrm{F}^{\mathrm{W}}(\omega) & =-\sigma_{\mathrm{n} 0} \frac{\mathrm{k}}{4 \pi} \frac{\Gamma_{\mathrm{r}}}{\Gamma} \frac{1}{2 \hbar\left(\omega-\omega_{0}\right) / \Gamma+\mathrm{i}} \\
& =-\sigma_{\mathrm{n} 0} \frac{\mathrm{k}}{4 \pi} \frac{\Gamma_{\mathrm{r}}}{\Gamma_{0}(1+\mathrm{W})} \frac{1}{2 \hbar\left(\omega-\omega_{0}\right) /\left((1+\mathrm{W}) \Gamma_{0}\right)+\mathrm{i}}
\end{aligned}
$$

Thus, all equations still apply with the substitutions of (2.30). In particular, one notes that the only effect of these on the forward scattering impulse response, (2.21), is to change the exponential decay time giving

$$
|G(t)|^{2} \rightarrow\left|G^{W}(t)\right|^{2}=\left|G^{W=0}(t)\right|^{2} e^{-W_{t} / \tau_{0}}
$$

The signal in a forward scattering time response measurement just goes as the integral of (2.32) over time $(t>0)$. It becomes smaller for increasing $W$, going as $1 /(1+W)$ for thin samples and more slowly for thicker samples. Thus, to see a forward scattering signal in time domain experiment, one would like to minimize the broadening.

The effects of broadening were particularly important in some recent work with the $6.2 \mathrm{keV}$ nuclear resonance of ${ }^{181} \mathrm{Ta}$ [Chumakov, et al., 1994, 27]. This transition has the advantage that the natural isotopic composition of $\mathrm{Ta}$ is nearly $100 \%{ }^{181} \mathrm{Ta}$, but the disadvantage that it is very narrow, $\Gamma_{0}=8 \times 10^{-11} \mathrm{eV}$ [Mouchel, et al., 1981, 28], about two orders of magnitude smaller than the ${ }^{57} \mathrm{Fe}$ resonance. Thus, it is very susceptible to the broadening effects mentioned above, and the natural line width has not been observed in a transmission experiment, with the best width observed (source+absorber) being about $15 \Gamma_{0}$ [Dornow, et al., 1979, 29].

The question in this case was, given that the resonance energy of Tantalum was only determined to within $\pm 20 \mathrm{eV}$ [Lederer and Shirley, 1978, 30], what is the best way to find a resonant signal using synchrotron radiation. In particular, we had a choice between samples that probably had a fairly broad line width (they had not been measured), and one sample known to have a fairly narrow line width [Dornow, et al., 1979, 29]. In short, a signal was finally seen in forward scattering from the narrow line sample, but, even having found the right 
energy, no signal was observable from the samples that having a broader line width. Parenthetically, one also notes that the transition energy was just outside the error bars of the accepted value.

\section{Incoherent Scattering}

The remarks in the previous section make it clear that when a line is substantially broadened, although the amount of absorption from the sample is enhanced (or remains the same), the amount of forward scattering is decreased. Of course, the absorbed energy has to go someplace, and this is into incoherent processes, e.g., internal conversion, or scattering with recoil. Throughout the bulk of this thesis, only coherent scattering is discussed. However, in light of some very recent developments it is worthwhile to discuss using synchrotron radiation to investigate incoherent scattering.

Synchrotron radiation has two features that allow incoherent scattering experiments to be done when they are not possible with conventional radioactive sources. The first is that it is pulsed, so, assuming one can gate in time, one may remove essentially all the background due to non-resonant scattering from the sample (as was suggested as early as 1962 [Seppi and Boehm, 1962, 31]). The second is that it is broad-band, so that it may be used to excite samples that have severely broadened resonances.

Thus, it was recently demonstrated that the nuclear resonant scattering from the $9.4 \mathrm{keV}$ resonance in gaseous ${ }^{83} \mathrm{Kr}$ may be observed using synchrotron radiation [Baron, et al., 1994, 32], or from ${ }^{57} \mathrm{Fe}$ ions in solution [Zhang, et al., 1994, 33]. On the one hand, up to the resolution of the monochromator used for the incident beam, this can allow one to map out velocity distributions of the resonant nuclei in a sample. On the other hand, there is potential to do perturbed angular correlation (PAC) ([Shirley and Haas, 1972, 34] [Mahnke, 1989, 35] ) studies using synchrotron radiation.

Perhaps most interesting, however, is that the analogous experiments performed with solids provide information about phonon densities of states. Thus, a collaboration in Japan [Seto, et al., 1994, 36] recently observed incoherent 
nuclear resonant scattering from a ${ }^{57} \mathrm{Fe}$ foil which showed structure at the meV level that may be associated with phonon effects. A group in France [Chumakov, 1994, 37] recently saw similar effects in the scattering from a powder sample of a large macromolecule. Though this author has only seen preliminary reports of this work, the potential of these experiments is great, and they certainly deserve mention.

\section{The Broad Bandwidth of Synchrotron Radiation}

Synchrotron radiation has many beautiful properties: it is well collimated, pulsed, polarized, and intrinsically broad band. This bandwidth, however, is much larger than is necessary for resonant nuclear experiments. Even after a standard Bragg reflection monochromator (i.e. a Si (111) Bragg reflection) the bandwidth is $\sim \mathrm{eV}$. The nuclear resonance width is $5 \times 10^{-9} \mathrm{eV}$ in ${ }^{57} \mathrm{Fe}$, so to a first approximation only about a part in $10^{8}$ of the incident radiation is useful. This makes for a very nasty signal to noise problem.

The saving grace for Mössbauer experiments is that synchrotron radiation arrives in pulses that are typically short ( $<1 \mathrm{~ns}$ ) relative to the nuclear lifetime. This is because the time distribution of the synchrotron radiation reflects the structure of the electrons in the storage ring, and the electrons, by virtue of the radio frequency ( $\mathrm{rf}$ ) acceleration techniques used in such machines are confined to small (short) bunches. Most electron storage rings used as synchrotron radiation sources may be run in a mode (sometimes called timing mode) where there are large dead times ( $>200 \mathrm{~ns}$ ) between successive electron bunches. The signal to noise problem in a nuclear scattering experiment then "reduces" to being able to separate a small signal (one photon) from a large signal that is slightly separated in time. This is just because the non-resonant background will be scattered quickly (and is called "prompt") while nuclear interactions lead to slower ("delayed") scattering.

Typical fluxes on the beamlines used for many of the resonant nuclear scattering experiments are $>\sim 10^{11}$ photons/second in the few $\mathrm{eV}$ bandwidth of the $\mathrm{Si}$ (111) monochromator (at $14.4 \mathrm{keV}$ ). Since the pulse rate is something like 5 
$\mathrm{MHz}$ (1/200 ns), this means that $>\sim 10^{4}$ photons are provided per pulse. Ideally, one would like to be able to detect a single $x$-ray sometime in the next $200 \mathrm{ns,}$ after a pulse of $>10^{4} x$-rays. At the present level of technology, this is difficult (especially at a $5 \mathrm{MHz}$ repetition rate). Thus, to actually do a synchrotron based experiment, one must either reduce the incident bandwidth further, or only investigate processes that favor nuclear scattering much more than they do electronic scattering, or both. Of course, one would like to also have the best detector available.

The initial solution to the problem was a careful choice of sample. In particular, some perfect crystals (e.g. yttrium iron garnet (YIG), iron borate, iron hematite) have structures in which the nuclear unit cell is larger than the electronic unit cell, thus providing the possibility to observe pure nuclear (or electronically forbidden) reflections. Thus, after the work of Gerdau, et al. [Gerdau, et al., 1985, 38] showed it was possible to see a signal in this sort of experiment, the first years of synchrotron Mössbauer work (1985-1989) used primarily pure nuclear reflections.

However, looking only at pure nuclear reflections in perfect crystals severely limits the possible choice of samples and the types of experiments that may be performed. Thus, there has been ongoing development both in optics to reduce the incident bandwidth and in detectors that can handle as large a prompt pulse as possible, and still recover to see a single photon event in a few $(<\sim 10) \mathrm{ns}$. Detector development has been an essential part of this thesis, and will be discussed in detail in chapter 3 . The optics, which are also crucial in these experiments will be discussed below.

\section{To Build a Better Monochromator}

The ideal monochromator for many nuclear resonant scattering experiments would have a bandwidth of something like 1 to $10 \mu \mathrm{eV}$. This is broad enough so that widely spaced nuclear resonances in a sample could be fully excited without affecting the resulting time development, and narrow enough to reduce the prompt background to easily manageable levels. There are two approaches to reaching this level, and unfortunately, neither is really ideal. 
On the one hand, the bandpass of conventional (electronic scattering) monochromators can be reduced by using high order Bragg reflections. However, this does not really approach the desired few $\mu \mathrm{eV}$ bandwidth, having a lower limit of a few meV. On the other hand, one can try to increase the bandwidth of the nuclear response of a sample (e.g. the speedup mentioned above) and, in fact, a nuclear scattering monochromator of bandwidth approaching the $\mu \mathrm{eV}$ level has been made. However, the monochromators based on nuclear scattering have the disadvantage that they usually do not have a flat frequency response over their bandpass, and are also difficult to scan. Both options are discussed in more detail below.

\section{Improved Conventional (Electronic Scattering) Monochromators}

Conventional electronic scattering techniques may be used to reduce the incident bandwidth in synchrotron radiation experiment to the level of a few meV. It turns out that, with modern detectors, this sufficiently reduces the prompt rate so that resonant nuclear scattering experiments may be performed. The optics devices are well explained using dynamical diffraction theory which is discussed, briefly, below. For more complete treatment of the dynamical diffraction, the reader is referred to the comprehensive text by James Uames, 1962 (Reprinted 1982),39] and the very nice review article by Batterman and Cole [Batterman and Cole, 1964, 40]. Colella [Colella, 1974, 41] also gives a general (numerical) method appropriate to some more complicated (multi-beam) problems.

The basic geometry for a Bragg reflection is shown in figure 2.4. If a beam of $\mathrm{x}$-rays (assumed, momentarily, to be perfectly collimated and monochromatic) is incident onto a perfect crystal at nearly the angle of a Bragg reflection, then the probability of reflection can be quite large, approaching unity. The geometry shown in figure 2.4 is appropriate for an asymmetric Bragg reflection in that the diffracting planes of the crystal are not parallel to the crystal surface. In the case that the planes are parallel to the surface, the reflection is called symmetric. The more general case is described here because it is of importance to the optics used in Mössbauer experiments. 


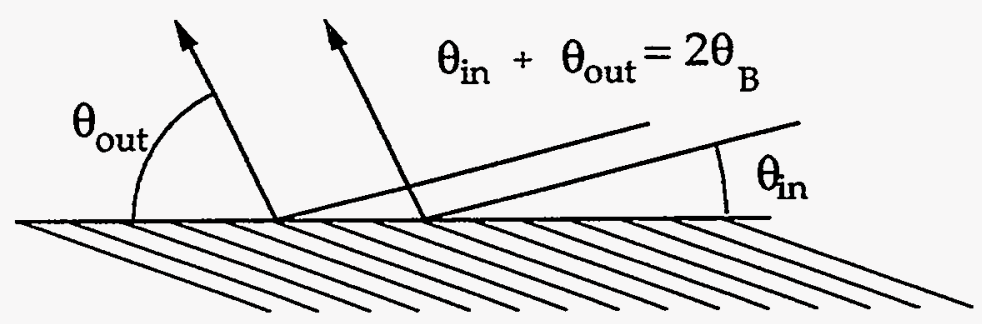

Figure 2.4. Geometry for an asymmetric Bragg reflection. The crystal planes are shown and are not parallel to the crystal surface.

The ratio of the reflected intensity to the incident intensity (again for a perfectly monochromatic, collimated beam) is given by [Batterman and Cole, $1964,40]^{*}$

$$
|R|^{2}=b^{2}\left|\eta \pm\left(\eta^{2}-1\right)^{1 / 2}\right|^{2}
$$

where

$$
\begin{aligned}
& \eta=\frac{b\left(\theta-\theta_{\mathrm{B}}\right) \sin \left(2 \theta_{\mathrm{B}}\right)+\delta(1-\mathrm{b})}{2|\mathrm{P}||\mathrm{b}|^{1 / 2}\left|\delta_{\mathrm{H}}\right|} \\
& \mathrm{b}=-\frac{\sin \theta_{\text {in }}}{\sin \theta_{\text {out }}} \\
& \lambda=2 d \sin \theta_{\mathrm{B}}
\end{aligned}
$$

$\mathrm{b}$ is the asymmetry parameter, $\mathrm{b}=-1$ for a symmetric Bragg reflection, and $\mathrm{P}$ is $\mathrm{a}$ polarization factor, $\mathrm{P}=1$ for sigma polarization (perpendicular to the scattering plane) and $P=\cos 2 \theta_{B}$ for pi polarization (in the scattering plane). The last relation is just Bragg's law. $\delta$ is the decrement of the index of refraction of the material from $1, \delta=1-n$. This is a measure of the forward scattering. $\delta_{H}$ is a measure of the scattering into the reflected beam. $\delta$ and $\delta_{\mathrm{H}}$ may be related to the appropriate scattering amplitudes for the crystal unit cell by a simple proportionality constant:

\footnotetext{
* Note that this form is not correct at grazing angle s of incidence near the critical angle. Also, we have assumed a centrosymmetric crystal.
} 


$$
\delta=-\frac{\lambda^{2}}{2 \pi \mathrm{V}} \mathrm{F}=\frac{\lambda^{2} \mathrm{r}_{\mathrm{e}}}{2 \pi \mathrm{V}} \mathrm{f}
$$

where $\mathrm{V}$ is the unit cell volume and $\mathrm{F}$ is the unit cell scattering amplitude. For electronic scattering problems, the classical radius of the electron is often factored out of the scattering amplitude and the oscillator strength in units of free electrons, $f$, is used instead. $f$ is approximately the number of electrons in the unit cell for forward scattering. An analogous relationship to 2.37 exists for $\delta_{H}$ with $F_{H}$ and $f_{H}$ replacing $F$ and $f . F_{H}$ is the just the unit cell scattering amplitude for the momentum transfer given by Bragg's law. In general, $\left|\delta_{\mathrm{H}}\right|<=\mid \delta 1$, and in the case of equality the reflection is sometimes called a full reflection.

$\delta$ and $\delta_{\mathrm{H}}$ are complex quantities, but the imaginary parts are typically small (see table 2.1). Thus, $\eta$ may be taken as approximately real and the region of high reflectivity is typically quoted as the range from $\eta=+1$ to $\eta=-1$ (since $b<0$, $\eta$ becomes smaller as angles become larger). The corresponding angular range is referred to as the Darwin width and is

$$
\Delta \theta_{\mathrm{D}}=\frac{4\left|\delta_{\mathrm{H}}\right||\mathrm{P}|}{\sin \left(2 \theta_{\mathrm{B}}\right)}\left(\frac{\sin \theta_{\text {out }}}{\sin \theta_{\text {in }}}\right)^{1 / 2}
$$

Figure 2.5 shows the (calculated) reflectivity as a function of angle for $14.4 \mathrm{keV}$ radiation near the Bragg angle for the $\mathrm{Si}(111)$ (symmetric) reflection. It has the characteristic Darwin-Prins shape, being basically flat topped, with slightly higher reflectivity at smaller angles. Table 2.1 Lists the relevant parameters for many of the reflections in Si that are useful for Mössbauer work*

\footnotetext{
* The inclusion of the $(840)$ reflection in the table is due to its polarizing properties.
} 


$\begin{array}{ccccccc}\text { Ref. } & \mathrm{d}(\AA) & \mathrm{E}(\mathrm{eV}) & \begin{array}{c}\theta_{\mathrm{B}} \\ \text { (Degrees) }\end{array} & \begin{array}{c}\Delta \theta_{\mathrm{D}} \\ (\mu \mathrm{rad})\end{array} & \Delta \mathrm{E} & \mathrm{f}_{\mathrm{H}} \\ (111) & 3.136 & 14413 & 7.884 & 18 & 1.9 \mathrm{eV} & (59.74,0.84) \\ (1064) & 0.4405 & " & 77.52 & 2.4 & 7.7 \mathrm{meV} & (12.28,0.55) \\ (1222) & 0.4405 & " & 77.52 & 2.4 & 7.7 \mathrm{meV} & (12.28,0.55) \\ (975) & 0.4362 & " & 80.38 & 2.1 & 5.2 \mathrm{meV} & (-8.50,-.38) \\ (777) & 0.4480 & " & 73.78 & 1.4 & 5.8 \mathrm{meV} & (9.07,0.39) \\ (422) & 1.109 & " & 22.83 & 5.9 & 0.20 \mathrm{eV} & (50.99,0.79) \\ (840) \sigma & 0.6072 & " & 45.10 & 2.0 & 28 \mathrm{meV} & (-23.70,-67) \\ \pi & " & " & " & 0.007 & 98 \mu \mathrm{eV} & \\ & & & & & & \\ (111) & 3.136 & 9404 & 12.14 & 29 & 1.3 \mathrm{eV} & (60.29,1.37) \\ (733) & 0.6635 & " & 83.47 & 17 & 19 \mathrm{meV} & (20.02,1.15) \\ & & & & & & \\ (111) & 3.136 & 6215 & 18.55 & 45 & 0.84 & (61.0,3.0) \\ (333) & 1.045 & " & 76.62 & 28 & 54 \mathrm{meV} & (-35.5,-2.8)\end{array}$

Table 2.1. Parameters for symmetric Bragg reflections in perfect silicon crystals. The energies correspond to those of the low lying nuclear excited states of $57 \mathrm{Fe}, 83 \mathrm{Kr}$ and ${ }^{181} \mathrm{Ta}$, respectively. Note that the forward scattering from silicon at these energies is given by $f(14413 \mathrm{eV})=(112.90,0.84), f(9400 \mathrm{eV})=(113.67,1.96)$ and $\mathrm{f}(6215 \mathrm{eV})$ $=(114.64,4.32)$. The notation $(\mathrm{a}, \mathrm{b})$ indicates a complex number with real part $a$ and imaginary part $b$. 


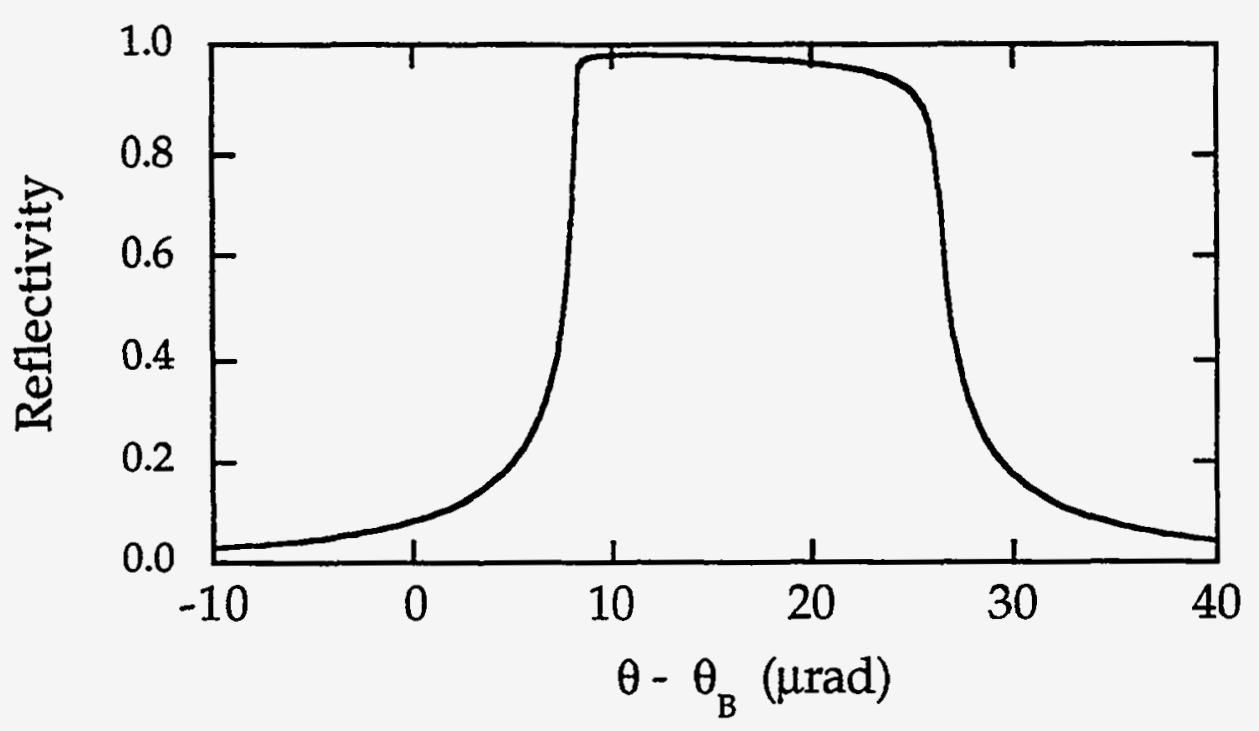

Figure 2.5. Reflectivity as function of angle for a symmetric $\mathrm{Si}$ (111) Bragg reflection at $14.4 \mathrm{keV}$ showing the standard Darwin-Prins shape. The offset from the exact (geometric) Bragg angle is due to refraction entering the silicon.

A synchrotron, however, is neither perfectly monochromatic nor perfectly collimated (on the scale of Bragg reflections). Thus, one must consider the effects of finite divergence and broad bandwidth. The Darwin width represents the angular acceptance of a crystal exposed to monochromatic radiation. This may be converted to an energy acceptance using Bragg's law, giving

$$
\begin{gathered}
\frac{\Delta \lambda}{\lambda}=\frac{\Delta \mathrm{E}}{\mathrm{E}}=\cot \theta_{\mathrm{B}} \Delta \theta_{\mathrm{D}}=\frac{2\left|\delta_{\mathrm{H}}\right| \mathrm{P} \mid}{\sin ^{2} \theta_{\mathrm{B}}}\left(\frac{\sin \theta_{\text {out }}}{\sin \theta_{\text {in }}}\right)^{1 / 2} \\
=\frac{4 \mathrm{~d}^{2} \mathrm{r}_{\mathrm{e}}|\mathrm{P}|}{\pi \mathrm{V}}\left(\frac{\sin \theta_{\text {out }}}{\sin \theta_{\text {in }}}\right)^{1 / 2}\left|\mathrm{f}_{\mathrm{H}}\right|
\end{gathered}
$$

The second equation has used (2.36) and (2.37) and is useful simply because, to a first approximation, $\mathrm{f}_{\mathrm{H}}$ is independent of energy so that the fractional energy width of a reflection is independent of energy.

It is very convenient for the purposes of describing optics for a synchrotron beamline to introduce DuMond diagrams, essentially a 2- 
dimensional figure showing the angular and energy acceptance of perfect crystal optics. Figure 2.6 shows the diagram for a single $\mathrm{Si}$ (111) reflection in a synchrotron beamline. The heavy vertical lines represent the beam from the synchrotron wiggler, which has a vertical angular divergence of $>\sim 100 \mu$ rads. The pair of diagonal lines represent the acceptance of a $\mathrm{Si}$ (111) reflection at 14.4 $\mathrm{keV}$. Thus it is easy to see the $\mathrm{Si}(111)$ reflection extracts a (correlated) range of angles and energies from the synchrotron beam. The energy width is $>\sim 10 \mathrm{eV}$ and the angular width is the full width of the synchrotron source.

Two facts are immediately obvious from figure 2.6. The first is that, from the point of view of Mössbauer experiments (which essentially use only radiation along a very thin horizontal stripe), there is a lot of extra radiation transmitted down the beamline. The second is that, at any given energy, there is a lot of radiation that is not reflected since it is not within the angular acceptance of the crystal. At a wiggler beamline with about $200 \mu \mathrm{rad}$ angular divergence (e.g. beamline 10-2 at SSRL), using a symmetric $\mathrm{Si}$ (111) monochromator reduces the (angle integrated) flux at any energy by about a factor of 10 at $14.4 \mathrm{keV}$. If the subsequent experiment is a diffraction experiment and only has limited angular acceptance, this is not a problem. However, if it is a Mössbauer forward scattering experiment, one has thrown away about a factor of 10 in intensity. 


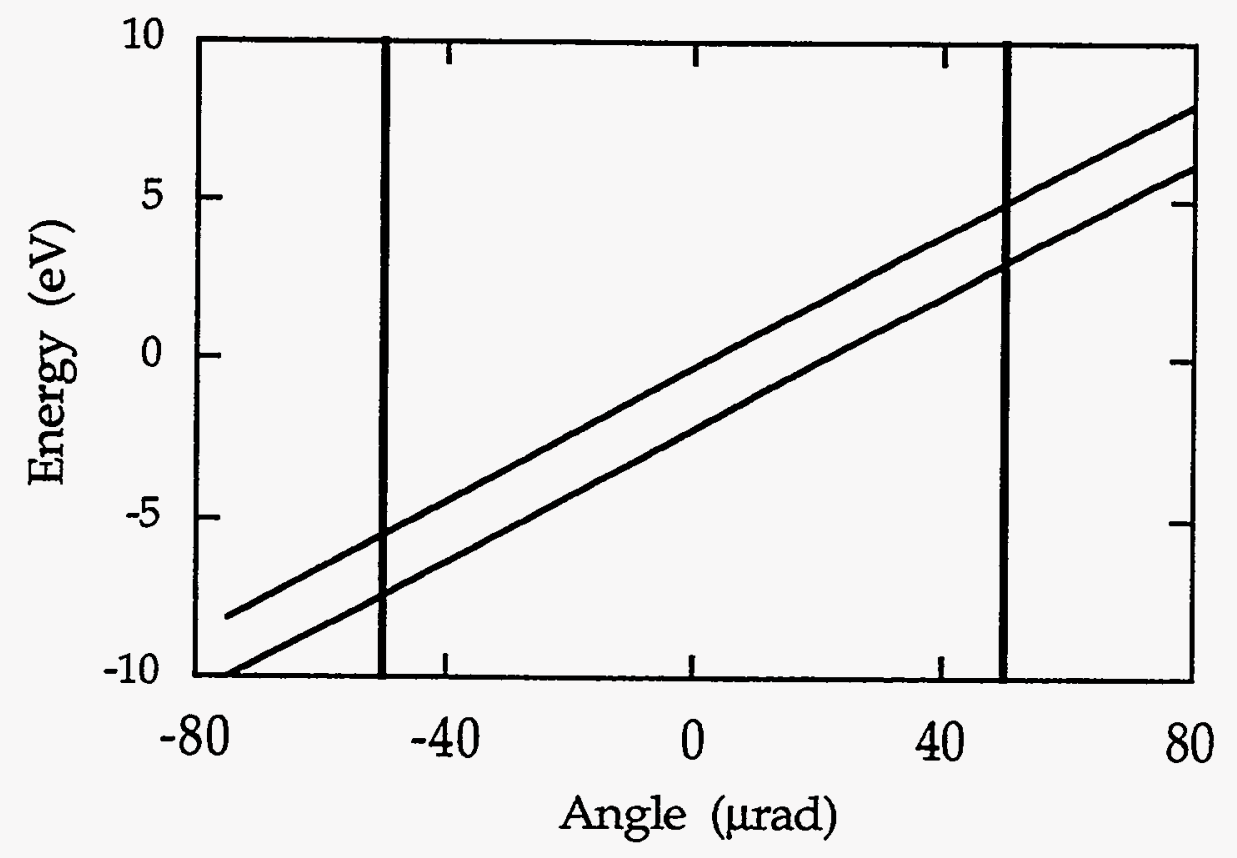

Figure 2.6. DuMond diagram for a Si(111) Bragg reflection at 14.4 $\mathrm{keV}$. The vertical lines represent the incident synchrotron radiation having a divergence of about $100 \mathrm{mrad}$ while the diagonal lines show the acceptance of the Si crystal.

Practically, the intensity loss at the resonance energy due to the limited acceptance of the standard $\mathrm{Si}(111)$ monochromator crystal (relative to the divergence of the synchrotron radiation) is just accepted. Many experiments are diffraction experiments, for which it is less important, and for the case of forward scattering experiments, the next level of optics, described below, reduces the angular acceptance even further, so it is not worth changing the $\mathrm{Si}(111)$ monochromator. At future beamlines (e.g. at ESRF, the APS and SPring-8) this problem will be corrected in two ways. First the source of the radiation will be an undulator on a high energy ring with very good emittance properties, so the source divergence will only be something like $25 \mu \mathrm{rad}$, comparable to the $\mathrm{Si}(111)$ Darwin width. Second since these will be beamlines dedicated to Mössbauer work the first $\mathrm{Si}$ (111) crystal may be modified to accept a broader angular range (see the discussion below). 
In 1987 Faigel, et al, [Faigel, et al., 1987, 42] made an important step toward improving the optics for synchrotron Mössbauer work. Simple investigation of Bragg's law shows that to maximize the angular acceptance for a given energy width of a reflection, it is best to choose a reflection with a Bragg angle near 90 degrees. Unfortunately, for low energies, the number of reflections possible is limited by the requirement that $\lambda>=2 d$, where $d$ is the distance between atomic Bragg planes, and for silicon at room temperature $d=5.432 \AA /\left(h^{2}+k^{2}+1^{2}\right)$ where $(\mathrm{h}, \mathrm{k}, \mathrm{l})$ are the indices of the reflection. Thus, some allowed reflections ${ }^{*}$ at 14.4 $\mathrm{keV}$ in Si that are close to 90 degrees are the (1064) and the (12 22) reflections having Bragg angles of 77.5 degrees and the $(975)$ reflection having a Bragg angle of 80.4 degrees (see table 2.1).

Faigel, et al, used a pair of symmetric energy dispersive (1064) reflections to further monochromatize the incident radiation. A single Bragg reflection essentially reflects a swath of radiation, as is shown in figure 2.6. If a second Bragg reflection identical to the first is also used, then (assuming both are symmetric) there are two distinct ways of aligning the second crystal with respect to the first. One can align them so the two crystal faces are parallel (nondispersive geometry), or one can align them so they have an angle between the two surfaces (dispersive geometry). This is shown in figure 2.7

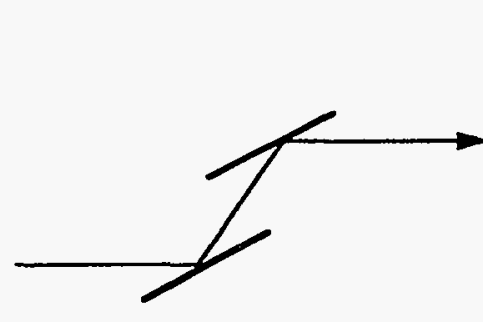

(a)

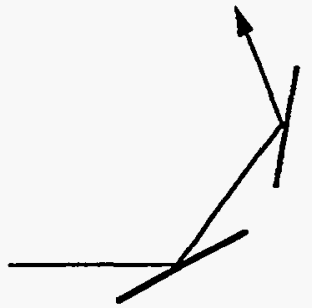

(b)

Figure 2.7. Non-dispersive (a) and dispersive (b) arrangements of a pair of Bragg reflections.

- Silicon is not a simple cubic lattice, but has a basis. It is a diamond lattice consisting (in real space) of two interpenetrating face-centered cubic lattices. Thus, not all $(h, k, l)$ reflections are allowed. The selection rules for allowed reflections are then that all of $h, k$ and $l$ must be odd, or they must all be even and their sum $(\mathrm{h}+\mathrm{k}+\mathrm{l})$ a multiple of four. 
Although the two arrangements are identical for perfectly monochromatic perfectly collimated beams, they have very different effects for divergent, polychromatic beams. A moments consideration will show that the nondispersive arrangement means that the DuMond plot of the acceptance of each crystal would be identical, while in a dispersive geometry, the acceptance region of the second crystal has the opposite slope to that of the first. Thus a pair of crystals in a dispersive geometry provide highly monochromatic, highly collimated beams, regardless of the source divergence. Finally, the fact that the beam from a single pair of crystals in a dispersive geometry leaves the crystals at some angle, not forward, is irritating so crystals are usually used in monolithic (for stability) channel cut pairs. The final geometry is then as shown in figure 2.8.

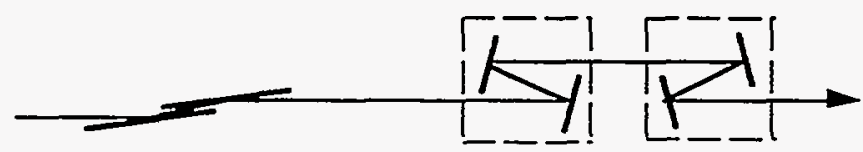

(a)

(b)

Figure 2.8. A Si (111) monochromator (non-dispersive arrangement) followed by a pair of channel cut (10 64) crystals arranged in a dispersive arrangement. This geometry was used by Faigel, et al [Faigel, et al., 1987, 42].

The resulting DuMond diagram is shown in figure 2.9. Note the expanded scale and that the heavy lines now correspond to the $\mathrm{Si}$ (111) acceptance. 


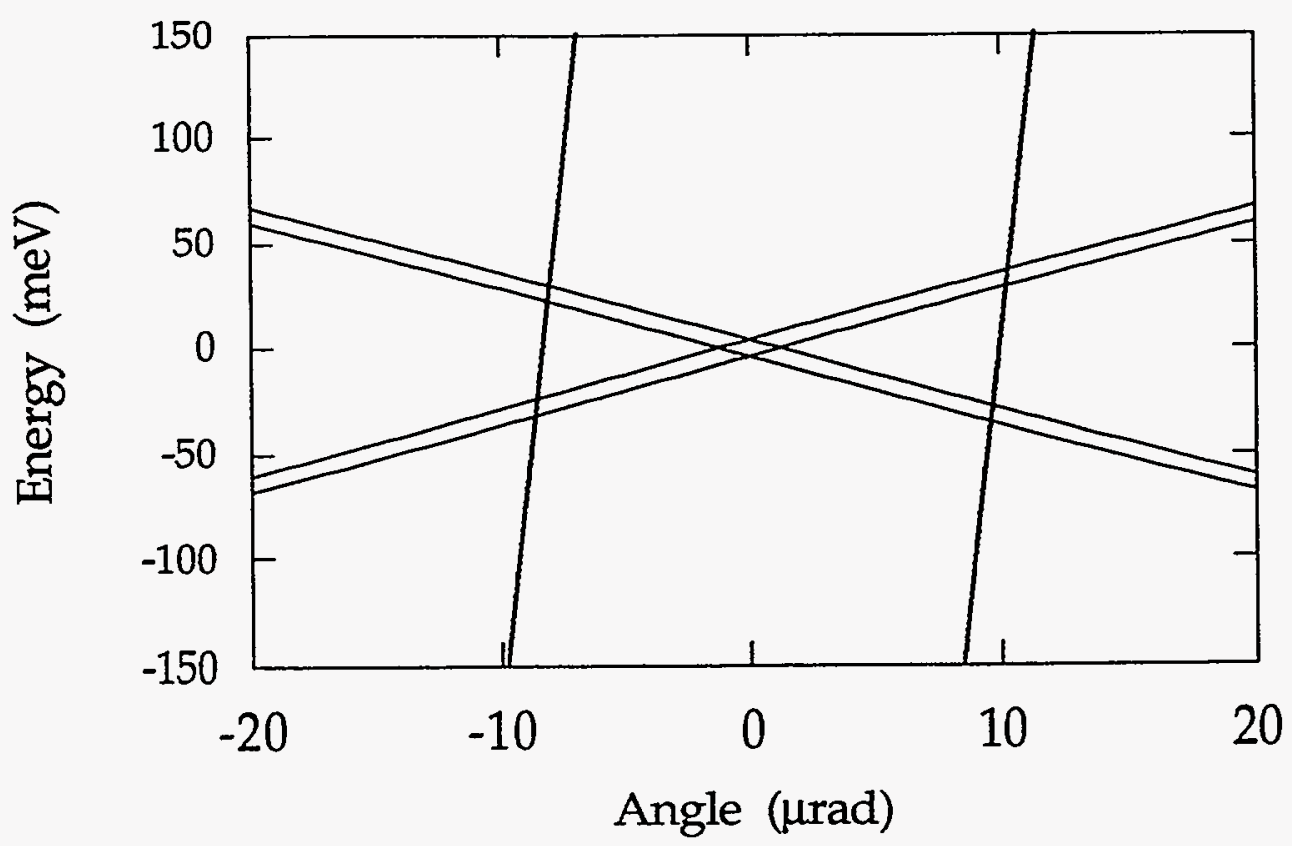

Figure 2.9. DuMond diagram for a Si (111) reflection followed by a pair of energy dispersive (10 64) reflections at $14,4 \mathrm{keV}$

The use of the two (1064) reflection then reduces the incident bandwidth to less than $10 \mathrm{meV}$ and the incident angular divergence to about $2 \mathrm{mrad}$, so the incident flux has been reduced by about 4 orders of magnitude (or more). However, in doing so one has only reduced the rate of resonant quanta by an order of magnitude (in the reduction of angular acceptance, relative to the $\mathrm{Si}(111)$ ). Though gaining three orders of magnitude in the signal to noise ratio is very desirable, the loss in signal of an order of magnitude (or more) is very irritating in low count rate experiment.

In 1992, Ishikawa, et al, [Ishikawa, et al., 1992, 43], suggested that asymmetric nested arrangement of crystals could be used to gain a larger angular acceptance and a device was built along these lines by Toellner, et al, [Toellner, et al., 1992, 44]. The basic idea here is that an asymmetric reflection can be used to collimate the incident beam. It is clear from equation (2.6) that if $\theta_{\text {out }} \neq \theta_{\text {in }}$ the angular acceptance of a crystal is changed. Furthermore, due to the symmetry of the equations, increasing the angular acceptance means that the angular divergence of the reflected beam will be lower than the incident beam, leading to collimation. Toellner, et al., used an asymmetrically cut $\mathrm{Si}$ (422) crystal to 
collimate the beam incident on a single $\mathrm{Si}(1064)$ channel cut. However, since the angular collimation of the beam also increases the beam size, as one might expect from Liouville's theorem (see figure 2.4), one also needs to reverse the collimation process to regain a small beam size. Thus a single symmetric channel cut (10 6 4) crystal was "nested" inside of a larger asymmetrically channel cut (4) 2 2) reflection.

The arrangement used by Toellner, et al., is shown in figure 2.10 . The asymmetry angle was 20 degrees $\left(\theta_{\text {in }}=2.8^{\circ}, \theta_{\text {out }}=42.8^{\circ}\right)$, leading to an angular acceptance of about $21 \mu \mathrm{rad}$, sufficient for the divergence from the $\mathrm{Si}$ (111) monochromator, and an output divergence of about $1.6 \mu \mathrm{rad}$, which is less then the acceptance of the (1064) reflection. In a DuMond diagram see figure 2.9. which does not show the spatial dimension, the incident stripe of the radiation from the $\mathrm{Si}(111)$ reflection is narrowed into a thin vertical band by the asymmetric (4 22 ) reflection. The symmetric (1064) reflection then accepts all of the photons of a given energy (within the angular bandwidth of the $\mathrm{Si}(111)$ ). The second (4 2 2) reflection then reduces the beam size, but increases the beam divergence to the same as it was before the nested monochromator.

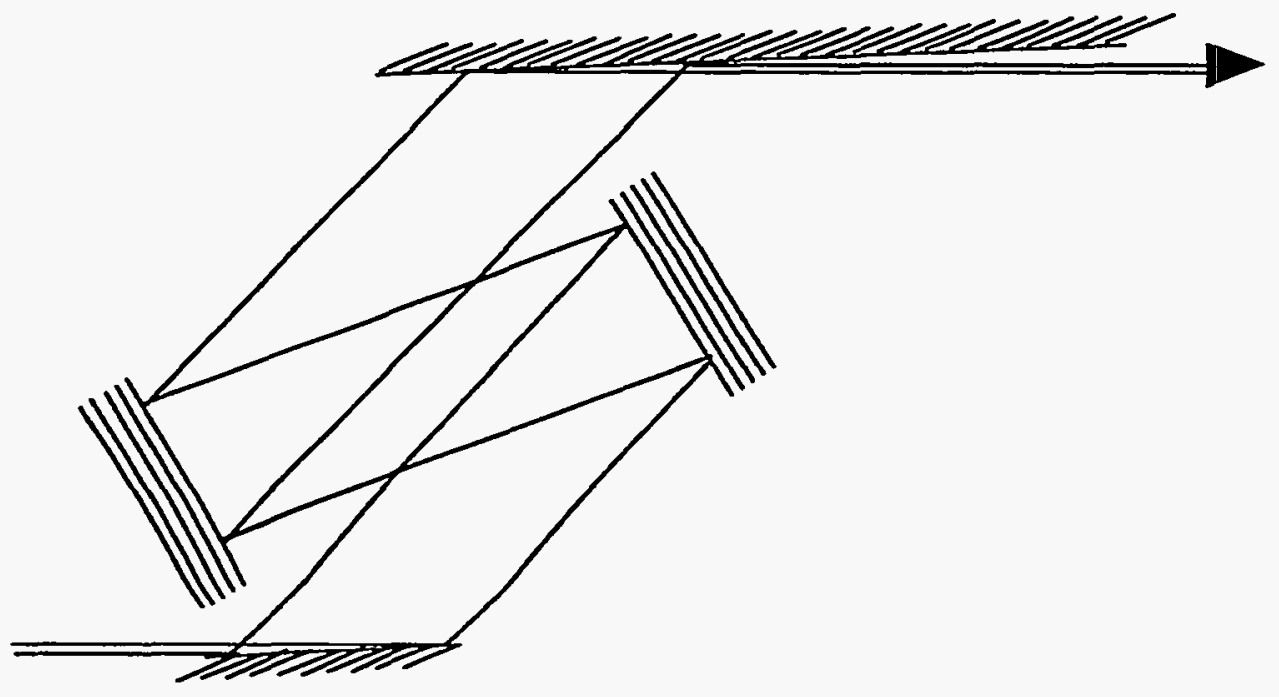

Figure 2.10 Asymmetric nested monochromator of Toellner, et al. [Toellner, et al., 1992, 44] The outer two reflections are from a single asymmetric channel cut $\mathrm{Si}\left(\begin{array}{l}4 \\ 2\end{array}\right)$ crystal while the inner pair are from asymmetric (10 64) crystal. The angles in the diagram are to scale. 
The experimental arrangement used in many present day nuclear scattering experiments with synchrotron radiation is then a $\mathrm{Si}(111)$ monochromator followed by a nested high resolution monochromator. This provides an incident bandwidth of about $10 \mathrm{meV}$ and angular divergence of about $20 \mu \mathrm{rad}$. In particular, the experiments described in chapter 6 of this thesis were carried out using the nested monochromator built by Toellner, et al.

\section{Monochromators using Nuclear Resonant Scattering.}

While high resolution monochromators using electronic scattering have become a common tool in resonant nuclear scattering experiments with synchrotron radiation, the use of monochromators based on resonant scattering is not common. This is primarily due to the fact that a high resolution electronic monochromator used with a good detector is sufficient to do most experiments. Making a monochromator based on resonant scattering requires both the materials (isotopically enriched) and the means (a deposition system), both of which are expensive. However, there has been a lot of effort to make monochromators based on resonant scattering, which merits discussion, and it is also very possible that they will prove useful in future experiments.

The important characteristics for a monochromator using nuclear scattering are that it should be fairly broad band (for ${ }^{57} \mathrm{Fe},>\sim 1 \mu \mathrm{eV} \sim 200 \Gamma_{0}$ ), that the frequency response within this band be fairly flat, that the reflectivity outside of the passband be small, and that it have a reasonable angular acceptance. Three approaches have been tried: pure nuclear Bragg reflections in perfect crystals; pure nuclear reflections from synthetic crystals or multilayers; grazing incidence anti-reflection (GIAR) films. In addition, there has also been some consideration of a Fresnel zone plate which might be useful when a small spot size is required[Mooney, et al., 1992, 45], but one has not been built.

Pure nuclear Bragg reflections in perfect crystals have extremely good rejection ratios, in that the reflectivity outside the passband is very small. However, the bandwidth, even with speedup effects from dynamical scattering, is usually less than $10 \Gamma_{0}$, limited in part by electronic absorption in the sample 
and in part by the small angular acceptance of the reflection ${ }^{*}$. Furthermore, the very structure that makes the reflection electronically forbidden usually affects the frequency response, so it is not flat. Attempts have been made to remove the structure in the frequency response, either by subsequent filtering of the reflected radiation [van Bürck, et al., 1990, 46] or by heating a crystal near the Neel temperature [Chumakov, et al., 1990, 47], with moderate success. However, no solution to the problem of a limited bandwidth has been found in naturally occurring crystals.

Pure nuclear reflections in synthetically grown multilayers are an attractive alternative to natural crystals. The artificial structure permits the nuclear environment to be tailored to remove complicated frequency response (e.g. hyperfine splitting) while the "pure nuclear" character of the reflection is preserved by selective isotopic enrichment (e.g. a ${ }^{57} \mathrm{Fe} /{ }^{56} \mathrm{Fe}$ superlattice has a pure nuclear reflection). Multilayer reflections also tend to have relatively large angular acceptances. However, the bandwidth of such structures, though larger than that of a pure nuclear reflection, is still limited, being about $40 \Gamma_{0}(0.2 \mathrm{meV})$ [Chumakov, et al., 1991, 48] [Chumakov, et al., 1993, 49] or 20Г 0 [Röhlsberger, et al., 1993, 50] depending upon the multilayer. In addition, because they operate at small angles of incidence ( $<10 \mathrm{mrad}$ ), there tends to be some specular reflection of the non-resonant radiation (non-resonant reflectivity $\sim 10^{-3}$, resonant reflectivity $\sim 10^{-1}$ ) so the rejection ratio is not nearly as good as for perfect crystals, though this may be improved somewhat in future work [Chumakov, $1993,51]$.

GIAR films provide the largest bandwidths of any of these methods. These devices rely on specular reflection from a resonant material. The nonresonant reflectivity is reduced through interference with a non-resonant overlayer or substrate. GIAR films were the first method considered specifically with synchrotron radiation in mind [Hannon, et al., 1979, 52]. and they have been the subject of considerable theoretical [Hannon, et al., 1985, 53, Hannon, et al., 1985, 54, Hannon, et al., 1985, 55, Hannon, et al., 1985, 56] and experimental [Grote, et al., 1989, 57, Grote, et al., 1991, 58, Röhlsberger, et al., 1992, 59, Alp, et al., 1993, 60, Röhlsberger, et al., 1993, 61] work. While the bandwidth for these

\footnotetext{
* The maximum speedup only occurs over a very small angular range at the center of the Bragg peak. Thus the angular acceptance with high bandwidth is very limited.
} 
devices can be large (measured value $\sim 110 \Gamma_{0}[$ Röhlsberger, et al., 1992, 59]), the grazing incidence geometry introduces structure into the reflectivity profile (see the previous refs. and [Gerdau, et al., 1990, 62]) which affects the time response of samples placed downstream [Röhlsberger, et al., 1992, 59, Röhlsberger, et al., 1993, 61]..

\section{References for Chapter 2}

1 H. Frauenfelder, The Mössbauer Effect. New York: W. A. Benjamin (1962).

2 D.H. Perkins, Introduction to High Energy Physics. Third ed. Menlo Park: Addison-Wesley (1987).

3 M. Lax, Multiple Scattering of Waves. Rev. Mod. Phys. 23 (1951) 287.

4 D.C. Hutchings, M. Sheik-Bahae, D.J. Hagan, and E.W. van Stryland, KramersKronig relations in nonlinear optics. Optical and Quantum Electronics 24 (1992) 1.

5 M. Weissbluth, Atoms and Molecules. San Diego: Academic Press (1978).

6 W. Heitler, The Quantum Theory of Radiation. Third ed. New York: Dover Publications, Inc. (1954).

7 E. Merzbacher, Quantum Mechanics. New York: John Wiley and Sons (1970).

8 J.S. Toll, Causality and the Dispersion Relation: Logical Foundations. Phys. Rev. 104 (1956) 1760.

9 Yu. Kagan, A.M. Afanas'ev, and V.G. Kohn, On Excitation of Isomeric Nuclear States in a Crystal by Synchrotron Radiation. J. Phys. C 12 (1979) 615.

10 M. Abramowitz and A. Stegun, ed. Handbook of Mathematical Functions. New York: Dover Publications, Inc. (1979).

11 U. van Bürck, D.P. Siddons, J.B. Hastings, U. Bergmann, and R. Hollatz, Nuclear Forward Scattering of Synchrotron Radiation. Phys. Rev. B 46 (1992) 6207. 
12 G.V.Smirnov, Yu.V. Shvyd'ko, and É. Realo, Accelerated Decay of a Nuclear Excitation in a Crystal During Resonant $\gamma$ Scattering. Pis'ma Zh. Eksp. Teor. Fiz. 39 (1984) 33.

13 R. Rüffer, E. Gerdau, R. Hollatz, and J.P. Hannon, Nuclear Bragg Scattering of Synchrotron Radiation Pulses in a Single-Reflection Geometry. Phys. Rev. Lett. 58 (1987) 2359.

14 U. van Bürck, R.L. Mössbauer, E. Gerdau, R. Rüffer, R. Hollatz, G.V. Smirnov, and J.P. Hannon, Nuclear Bragg Scattering of Synchrotron Radiation with Strong Speedup of Coherent Decay, Measured on Antiferromagnetic ${ }^{57} \mathrm{FeBO}_{3}$. Phys. Rev. Lett. 59 (1987) 355.

15 A.Q.R. Baron, J. Arthur, S.L. Ruby, D.E. Brown, A.I. Chumakov, G.V. Smirnov, G.S. Brown, and N.N. Salashchenko, The Time Response of a Thin Film of $57 \mathrm{Fe}$ Excited by Synchrotron radiation at Grazing Incidence. Presented at the International Conference on Anomalous Scattering, Malente, Germany (1992).

16 G.T. Trammell, Gamma Ray Diffraction by Resonant Nuclei, in Chemical Effects on Nuclear Transformations, Editor. Vienna: International Atomic Energy Agency (1961) p. 75.

17 G.T. Trammell and J.P. Hannon, Comment on "N Dependence of Coherent Radiation from Crystals". Phys. Rev. Lett. 61 (1988) 653.

18 J.P. Hannon and G.T. Trammell, Coherent Excitations of Nuclei in Crystals by Synchrotron Radiation Pulses. Physica B 159 (1989) 161.

19 G.T. Trammell and J.P. Hannon, Quantum Beats from Nuclei Excited by Synchrotron Pulses. Phys. Rev. B 18 (1978) 165.

20 G.T. Trammell and J.P. Hannon, Erratum: Quantum beats from nuclei excited by synchrotron pulses. Phys. Rev. B 19 (1979) 3835.

$21 \mathrm{Yu}$. Kagan and A.M. Afanas'ev, Coherence effects during nuclear resonant interaction of gamma quanta in perfect crystals, in Mössbauer Spectroscopy and Its Applications, Editor. Vienna: International Atomic Energy Co,mission (1972) p. 143.

22 U. van Bürck, G.V. Smirnov, R.L. Mössbauer, H.J. Marus, and N.A. Semioschkina, Enhanced Nuclear Resonance Scattering in Dynamical Diffraction of Gamma Rays. J. Phys. C 13 (1980) 4511. 
23 S.L. Ruby and D.I. Bolef, Acoustically Modulated Gamma Rays from ${ }^{57} \mathrm{Fe}$. Phys. Rev. Lett. 5 (1960) 5.

24 Yu.V. Shvyd'ko, A.I. Chumakov, G.V. Smirnov, V.G. Kohn, T. Hertrich, U. van Bürck, E. Gerdau, H.D. Rüter, J. Metge, and O. Leopold, Nuclear Bragg Diffraction of Synchrotron Radiation in the Presence of Acoustic Vibrations. Europhys. Lett. 22 (1993) 305.

25 U. van Bürck, G.V. Smirnov, J. Arthur, S.L. Ruby, A.I. Chumakov, A.Q.R. Baron, and G.S. Brown, Ultrasonic echo in time domain experiments. Work in progresss. (1994).

26 Yu.V.Shvyd'ko, K. Ebner, H.D. Rüter, J. Metge, E. Gerdau, and A.I. Chumakov, Mössbauer-Radiofrequency Double Resonance with Synchrotron Light, Seeheim, Germany: Fourth Seeheim Workshop on Mossbauer Spectroscopy (unpublished). (1994).

27 A.I. Chumakov, A.Q.R. Baron, J. Arthur, S.L. Ruby, G.S. Brown, G.V. Smirnov, U. van Bürck, and G. Wortmann, Nuclear scattering of Synchrotron Radiation by ${ }^{181} \mathrm{Ta}$. In Preparation (1994).

28 D. Mouchel, A.N. Larsen, and H.H. Hansen, Half-Life of the $6.21 \mathrm{keV}$ Level in ${ }^{181}$ Ta. Zeitschrift für Physik A 300 (1981) 85.

29 V.A. Dornow, J. Binder, A. Heidemann, G.M. Kalvius, and G. Wortmann, Preparation of narrow-line sources for the $6.2 \mathrm{keV}$ Mössbauer resonance of ${ }^{181} \mathrm{Ta}$. Nucl. Instrumen. and Meth. 163 (1979) 491.

30 C.M. Lederer and V.S. Shirley, ed. Table of Isotopes. New York: John Wiley and Sons (1978).

31 E.J. Seppi and F. Boehm, Nuclear Resonance Excitation Using a Diffraction Monochromator. Phys. Rev. 128 (1962) 2334.

32 A.Q.R. Baron, A.I. Chumakov, S.L. Ruby, J. Arthur, G.S. Brown, G.V. Smirnov, and U. van Bürck, Nuclear Resonant Scattering of Synchrotron Radiation by Gaseous Krypton. (1994) Submitted for Publication.

33 X.W. Zhang, Y. Yoda, M. Seto, M. Ando, and S. Kikuta, Nuclear Excitation of 57Fe Ion in the HCl Liquid by Synchrotron Radiation. (1994) In preparation.

34 D.A. Shirley and $\mathrm{H}$. Haas, Perturbed angular correlation of gamma rays. Annual Review of Physical Chemistry 23 (1972) 385.

35 H.E. Mahnke, Introduction to PAC/PAD. Hyp. Int. 49 (1989) 77. 
36 M. Seto, Y. Yoda, S. Kikuta, X.W. Zhang, and M. Ando, Observation of Nuclear Resonant Scattering Accompanied by Phonon Excitation using Synchrotron Radiation. (1994) Submitted for Publication.

37 A.I. Chumakov, private communication (1994).

38 E. Gerdau, R. Rüffer, H. Winkler, W. Tolksdorf, C.P. Klages, and J.P. Hannon, Nuclear Bragg Diffraction of Synchrotron Radiation in Yttrium Iron Garnet. Phys. Rev. Lett. 54 (1985) 835.

39 R.W. James, The Optical Principles of the Diffraction of X-rays. Woodbridge, Connecticut: Ox Bow Press (1962 (Reprinted 1982)).

40 B.W. Batterman and H. Cole, Dynamical Diffraction of X-Rays by Perfect Crystals. Rev. Mod. Phys. 36 (1964) 681.

41 R. Colella, Multiple Diffraction of X-rays and the Phase Problem. Computational Procedures and Comparison with Experiment. Acta Cryst. A30 (1974) 413.

42 G. Faigel, D.P. Siddons, J.B. Hastings, P.E. Haustein, J.R. Grover, J.P. Remeika, and A.S. Cooper, New Approach to the Study of Nuclear Bragg Scattering of Synchrotron Radiation. Phys. Rev. Lett. 58 (1987) 2699.

43 T. Ishikawa, Y. Yoda, K. Izumi, C.K. Suzuki, X.W. Zhang, M. Ando, and S. Kikuta, Construction of a precision diffractometer for nuclear Bragg scattering at the Photon Factory. Rev. Sci. Instrumen. 63 (1992) 1015.

44 T.S. Toellner, T. Mooney, S. Shastri, and E.E. Alp. High energy resolution, high angular acceptance crystal monochromator. in Optics for High-Brightness Synchrotron Beamlines. J. Arthur ed. SPIE Vol 1740, 1992. p. 218.

45 T.M. Mooney, E.E. Alp, and W.B. Yen, Mössbauer-Fresnel Zone Plate. J. Appl. Phys. 71 (1992) 5709.

46 U. van Bürck, R.L. Mössbauer, E. Gerdau, W. Sturhahn, H.D. Rüter, R. Rüffer, A.I. Chumakov, M.V. Zelepukhin, and G.V. Smirnov, Shaping of Nuclear Bragg Reflected SR-Pulses by Broad Resonance Absorbers. Europhys. Lett. 13 (1990) 371.

47 A.I. Chumakov, M.V. Zelepukhin, G.V. Smirnov, U. van Bürck, R. Rüffer, R. Hollatz, H.D. Rüter, and E. Gerdau, Time Spectra of a Nearly-Single-Line Pure Nuclear Reflection Excited by Synchrotron Radiation. Phys. Rev. B41 (1990) 9545. 
48 A.I. Chumakov, G.V. Smirnov, S.S. Andreev, N.N. Salashchenko, and S.I. Shinkarev, Diffraction of nuclear gamma-radiation by a synthetic multilayer structure. Pis'ma Zh. Eksp. Teor. Fiz [JETP Lett. 54, 216 (1991)] 54 (1991) 220.

49 A.I. Chumakov, G.V. Smirnov, A.Q.R. Baron, J. Arthur, D.E. Brown, S.L. Ruby, G.S. Brown, and N.N. Salashchenko, Resonant Diffraction of Synchrotron Radiation by a Nuclear Multilayer. Phys. Rev. Lett. 71 (1993) 2489.

50 R. Röhlsberger, E. Witthoff, and E. Gerdau, Observation of nuclear diffraction from multilayers with $\mathrm{Fe} \mathrm{F}^{57} \mathrm{Fe}$ superstructure. J. Appl. Phys. 74 (1993) 1933.

51 A.I. Chumakov, private communication (1993).

52 J.P. Hannon, G.T. Trammell, M. Mueller, E. Gerdau, H. Winkler, and R. Rüffer, Impedance-Matched Grazing Incidence Films: Pure Nuclear Reflections, Resonant Filtering of Synchrotron Radiation and X-Ray Interferometry. Phys. Rev. Lett. 43 (1979) 636.

53 J.P. Hannon, N.V. Hung, G.T. Trammell, E. Gerdau, M. Mueller, R. Rüffer, and $\mathrm{H}$. Winkler, Grazing-incidence Antireflection films I: Basic theory. Phys. Rev. B 32 (1985) 5068.

54 J.P. Hannon, N.V. Hung, G.T. Trammell, E. Gerdau, M. Mueller, R. Rüffer, and H. Winkler, Grazing-incidence Antireflection films II: Alternate techniques and General multilayer theory. Phys. Rev. B 32 (1985) 5081.

55 J.P. Hannon, G.T. Trammell, M. Mueller, E. Gerdau, R. Rüffer, and H. Winkler, Grazing-Incidence Antireflection films III: General theory for pure nuclear reflections. Phys. Rev. B 32 (1985) 6363.

56 J.P. Hannon, G.T. Trammell, M. Mueller, E. Gerdau, R. Rüffer, and H. Winkler, Grazing-Incidence Antireflection Films. IV. Application to Mössbauer Filtering of Synchrotron Radiation. Phys. Rev. B32 (1985) 6374.

57 M. Grote, R. Röhlsberger, E. Gerdau, R. Hellmich, U. Bergmann, M. Harsdorff, M. Chambers, and W. Pfützner, Preparation and Characterization of GLAR-Films for Monochromatization of Synchrotron Radiation. Hyp. Int. 58 (1989) 2439.

58 M. Grote, R. Röhlsberger, M. Dimer, E. Gerdau, R. Hellmich, R. Hollatz, J. Jäschke, E. Luken, R. Rüffer, H.D. Rüter, W. Sturhahn, E. Witthoff, M. Harsdorff, W. Pfützner, M. Chambers, and J.P. Hannon, Nuclear Resonant Filtering of Synchrotron Radiation by Grazing-Incidence Antireflection Films. Europhys. Lett. 17 (1991) 707. 
59 R. Röhlsberger, E. Gerdau, M. Harsdorff, O. Leupold, E. Lüken, J. Metge, R. Rüffer, H.D. Rüter, W. Sturhahn, and E. Witthoff, Broad-band Nuclear Resonant Filters for Synchrotron Radiation: a New Source for Nuclear Diffraction Experiments. Europhys. Lett. 18 (1992) 561.

60 E.E. Alp, T.M. Mooney, T. Toellner, W. Sturhahn, E. Witthoff, R. Röhlsberger, E. Gerdau, H. Homma, and M. Kentjana, Time Resolved Nuclear Resonant Scattering from ${ }^{119}$ Sn Nuclei using Synchrotron Radiation. Phys. Rev. Lett. 70 (1993) 3351.

61 R. Röhlsberger, E. Gerdau, E. Lüken, H.D. Rüter, J. Metge, and O. Leupold, Nuclear diffraction experiments with grazing incidence anti-reflection films. Zeitschrift Für Physik B B92 (1993) 489.

62 E. Gerdau, M. Grote, and R. Röhlsberger, Computer Simulations of GIAR-Film Spectra. Hyp. Int. 58 (1990) 2433. 


\section{Detectors}

\section{Introduction}

This chapter describes work to build a better detector for nuclear scattering experiments using synchrotron radiation. The work began as an attempt to build a nearly ideal (but complicated) detector based on microchannel plates (MCPs) and ended up with the development of a detector based on large area avalanche photodiodes (APDs)*. Though finally the MCP work was abandoned, the basic concept is described, and some of the useful results that came out of the work. The bulk of the chapter is devoted to presenting the work with the APDs, which was very successful. APD detectors are now being used for resonant nuclear scattering experiments by several groups, both in the United States and Europe, as well as Japan.

Resonant nuclear scattering experiments place fairly stringent requirements on the detectors used. The detector wish list is:

(1) Good detection efficiency ${ }^{+}$for single $x$-rays ( $>10 \%$, preferably $>50 \%$ ).

(2) Low background ( $<\sim 0.1 \mathrm{cts} / \mathrm{sec}$, preferably $<0.01 \mathrm{cts} / \mathrm{sec})$.

(3) Good time resolution ( $<\sim 1 \mathrm{~ns})$.

(4) Fast recovery time/large dynamic range.

The first two just put some relevant numbers on what one would like in any single photon counting experiment, while the second two are somewhat novel. The time resolution requirement is finally not too difficult to fulfill. The last requirement, however, is both unique to these experiments and difficult to satisfy.

\footnotetext{
* The bulk of avalanche diode work by this author has been, or will be,published [Baron and Ruby, 1993, 1][Baron, 1994, 2].

+ Throughout this chapter and this thesis, unless otherwise indicated, "efficiency" will mean the probability of an incident photon generating a pulse from the relevant detector. It is not to be confused with "quantum efficiency" which is the average number of electrons generated per incident photon.
} 
The dynamic range problem was introduced in the previous chapter. Synchrotron radiation arrives in pulses of short $(<1 \mathrm{~ns})$ duration at repetition rates that vary from about 1 to $5 \mathrm{MHz}$ (depending on the operating conditions at the storage ring). Nuclear resonant scattering events are separated from electronic scattering events by gating in time: electronic scattering occurs on the femtosecond time scale, and is called "prompt", while nuclear resonant scattering (for the resonances discussed in this thesis) occurs on the 10 to 100 nanosecond time scale, and is called "delayed". The prompt rate is usually many orders of magnitude greater than the delayed rate ${ }^{*}$ so that some sort of gating is usually necessary.

For the purposes of detector work, there are three classes of experiments, depending of the prompt count rate. At one extreme is the case that the prompt rate is much less than the synchrotron pulse rate (much less than 1 to $5 \mathrm{MHz}$ ). In this case, if the detector requires a microsecond, or even a few microseconds to recover after a prompt event, the experiment is not affected because the fraction of time the detector is blind is very small. In the middle range, the prompt rate approaches the synchrotron pulse rate. This adds two complications: first, the detector must be able to recover quickly from a single photon event or it will be paralyzed by the recurring prompt pulses; and, second, it is more probable that a single pulse may have multiple photons in it. The latter means the signal from the detector must be fairly clean, without large reflections or ringing, so that a discriminator will not be multiply triggered by large events. Finally, there is the regime where there is almost always at least one prompt photon detected per pulse and possibly many. Here one will be limited by the response time of the detector, and any sort of ringing in the detector response will be deadly.

\section{The Plastic Scintillation Coincidence Detector}

When this work began, the detector used in resonant nuclear scattering experiments with synchrotron radiation at SSRL was a plastic scintillation

\footnotetext{
* The experiments this author has done have had ratios (prompt rate/delayed rate) varying from about $10^{2}$ to $10^{8}$. However, by using a pure nuclear Bragg reflection and a high resolution monochromator, it is possible to make the ratio (delayed rate/prompt rate) unity, or better[Faigel, et al., 1987,3]
} 
coincidence detector, shown in figure 3.1. This design was developed independently by groups at SSRL and at DESY in Hamburg, Germany. The design is driven by two concerns: the low light output of plastic scintillator and the thermal noise of photomultiplier tubes.

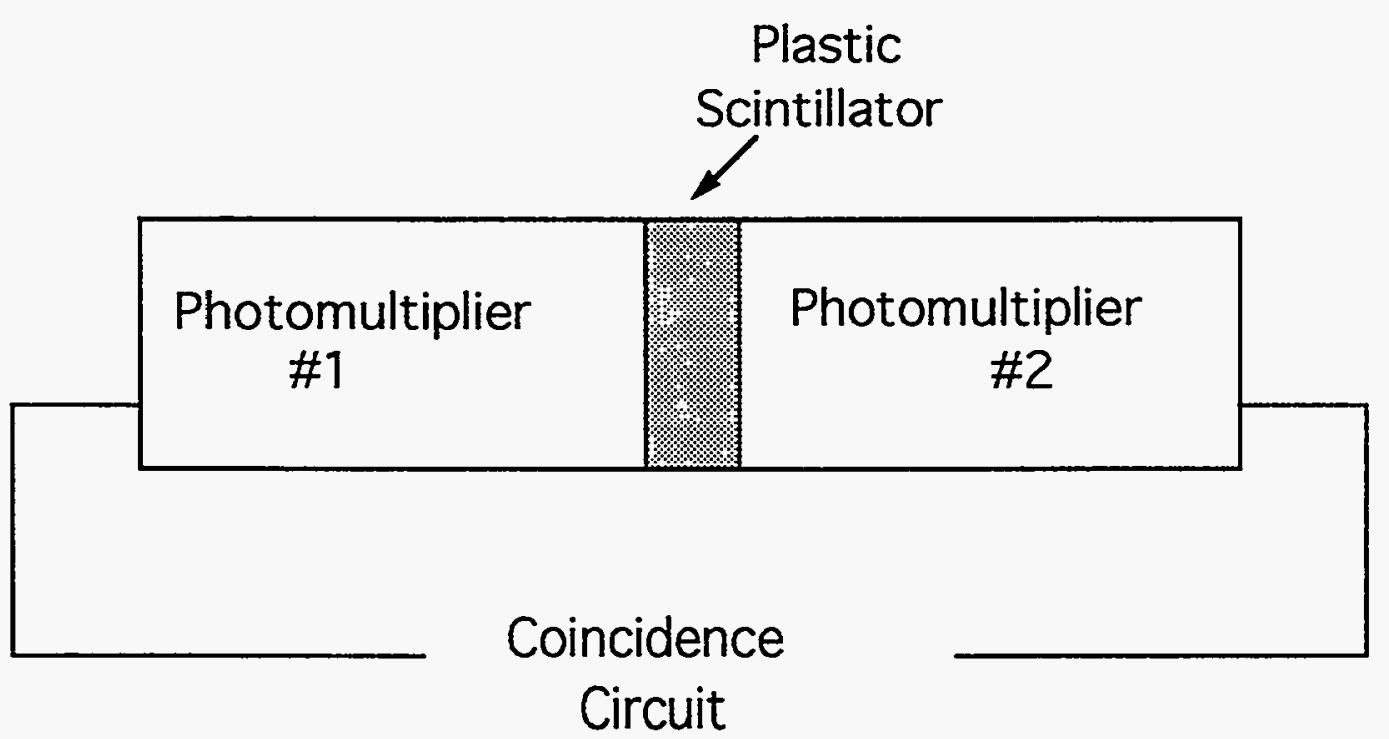

Figure 3.1 Schematic of a plastic scintillator coincidence detector.

The choice of plastic scintillator as a converter from $x$-ray to visible wavelengths is determined by the time resolution requirements of the experiment. Other scintillation materials (e.g. $\mathrm{NaI}(\mathrm{Tl})$ ) have higher visible light outputs, but poorer time resolution, while some materials (e.g. $\mathrm{BaF}_{2}{ }^{*}$ ) may have good time resolution but very low light output. The reader is referred to the catalogues of the various companies that produce scintillation materials for more information (e.g. [Bicron, 4]). In general, one notes that synchrotron radiation experiments tend to operate at the low end of energies for which scintillation detectors are commonly used, thus light output is an important concern.

A coincidence detector is used to reduce the noise. Thermal emission rates from the photocathode or early dynodes of the photomultiplier are of order 100

\footnotetext{
* $\mathrm{BaF}_{2}$ also has a slow light component which is larger than the fast component and would probably be a significant problem in high rate experiments.
} 
$\mathrm{Hz}$. Unless one sets the discriminator threshold significantly above a single electron event, there will be noise at the $100 \mathrm{~Hz}$ level from thermal emission. Unfortunately, the light output for the low energy $x$-rays used in these experiments is small enough so that the efficiency will be significantly reduced if the discriminator is set high enough to reject thermal noise events. Instead, two photomultipliers are used in coincidence, reducing the thermal noise rate to about $0.2 \mathrm{~Hz}$. Potentially, another solution might be to cool the photomultipliers, and this has been tried (without coincidence techniques) with reduction of the noise from tens of counts/sec to a few counts/sec by using dry ice [Arthur, 1994, 5].

The coincidence detector built at SSRL by S.L. Ruby and J. Arthur used Bicron 420 plastic scintillator [Bicron, 4] and Hamamatsu R329 photomultiplier tubes with E934 bases [Hamamatsu, 6] . It had an efficiency of about $35 \%$ for detection of $14.4 \mathrm{keV} x$-rays, a noise rate of about $0.2 \mathrm{~Hz}$ and a time resolution of about $2.5 \mathrm{~ns}$ (including the jitter in the electronics). A similar detector, built in Hamburg [Metge, et al., 1990, 7] (but using different photomultiplier tubes, the XP2020 [Philips Components, 1989, 8]) had a time resolution of about $1.3 \mathrm{~ns}$, but the absolute noise rate was not clearly specified (the implication of the paper is that it was $\sim 1 \mathrm{~Hz}$ ) and no mention was made of the efficiency.

Finally, we consider the maximum allowable prompt rates (or dynamic range) for these detectors. The SSRL detector was limited to prompt count rates of $<\sim 0.3$ photons/bunch (or $\sim 100 \mathrm{kHz}$ prompt count rate at the PEP storage ring). At these rates, the electronic ringing of the signal (probably from multiple photon events) in the photomultiplier base became large enough that spurious peaks were introduced into the data. Searching the published results from other experiments, it seems that this rate ( $<\sim .3$ detected photons/bunch) is the typical upper limit for this style of detector. However, some recent work [Bergmann, et al., 1994, 9] used a similar style plastic coincidence detector (but with R2083 photomultiplier tubes) at count rates approaching 2 or 3 detected photons/pulse $(\sim 2 \mathrm{MHz}$ pulse rate) [Shastri, 1994, 10]. The recovery time for the detector at these high rates was as long as 30 to $40 \mathrm{~ns}$, and there was some evidence of afterpulsing, but it is clear that maximum allowable prompt rate is not as limited as the experience at SSRL might indicate. It is also worth mentioning that even 
plastic scintillation materials can have a delayed light component that, although small ${ }^{*}$, might be a consideration at very high rates.

\section{A Microchannel Plate (MCP) Detector.}

The hope for a microchannel plate (MCP) detector was to jump directly. from the low prompt rate domain well into the high prompt rate range, possibly approaching count rates of $10^{5}$ prompt photons/pulse. (For an introduction to MCPs, see [Wiza, 1979, 12] and [Eberhardt, 1979, 13]) On the one hand it seemed that it might just be possible and, on the other, it seemed necessary to justify the added overhead (in time and equipment) of using microchannel plates. It was hoped that a sufficiently good detector might make it unnecessary to have a high resolution monochromator of the type described in the previous chapter. ${ }^{+}$

The driving concern in building the $\mathrm{MCP}$ detector, and the essential fact distinguishing it from the APD detector described later, was gating the detector before the electron gain took place. This avoids dealing with currents that can approach the ampere level at high rates, and the difficulty of distinguishing an electronic signal corresponding to a single photon on the tail of one corresponding to $10^{4}$ or $10^{5}$ photons. The work was motivated by the relatively recent development of a fast gated microchannel plate photomultiplier tube [Hamamatsu, 6], the sub-nanosecond images being made of fusion events using microchannel plates [Kilkenny, 1991, 15] and the large interest of the astrophysics community in low noise microchannel plates for UV imaging [Siegmund, et al., 1988, 16].

The basic concept behind this detector was the use of microchannel plates as amplifying elements for electrons ejected from an $x$-ray photocathode material (either Au or CsI). It is possible to get reasonable ( $\sim 50 \%)$ efficiencies from these materials by using grazing angles of incidence ( 0.5 degrees, see below). Figure 3.2 shows a schematic of the detector that was built. The microchannel plates

\footnotetext{
* In Bicron 420 there is a delayed component at $15 \mathrm{~ns}$ after excitation that is about 0.02 of the faster ( ns lifetime) component [Ruby and Tirsell, 1992,11].

+ Another type of MCP detector has been developed in Hamburg by Klaus Quast for the detection of conversion electrons. This was not designed to survive extremely high prompt rates but was optimized for good time resolution and low background rates [Gerdau, 1993, 14].
} 
were made by Galileo [Galileo Electro-Optics, 17] and were 2" in diameter (40 $\mathrm{mm}$ diameter active area) with $10 \mu \mathrm{m}$ pores in $0.5 \mathrm{~mm}$ thick glass. The upper microchannel plate (nearest the cathode) was coated with $1000 \AA$ of $\mathrm{Au}$ for a conducting contact while the other was coated with $300 \AA$. The resistances of the plates were about 30 and $70 \mathrm{meg}$, respectively, and they were typically operated at voltages of 0.9 to $1.2 \mathrm{kV}$, with gains of about $10^{3}$ (for each). A nickel mesh grid (90 wires/inch, 90\% transmission [Buckbee-Mears, 18] ) was placed between the photocathode and the first microchannel plate (the dashed line in figure 3.2). All bias voltages (cathode, grid, each $\mathrm{MCP}$ and anode) were independently controlled.
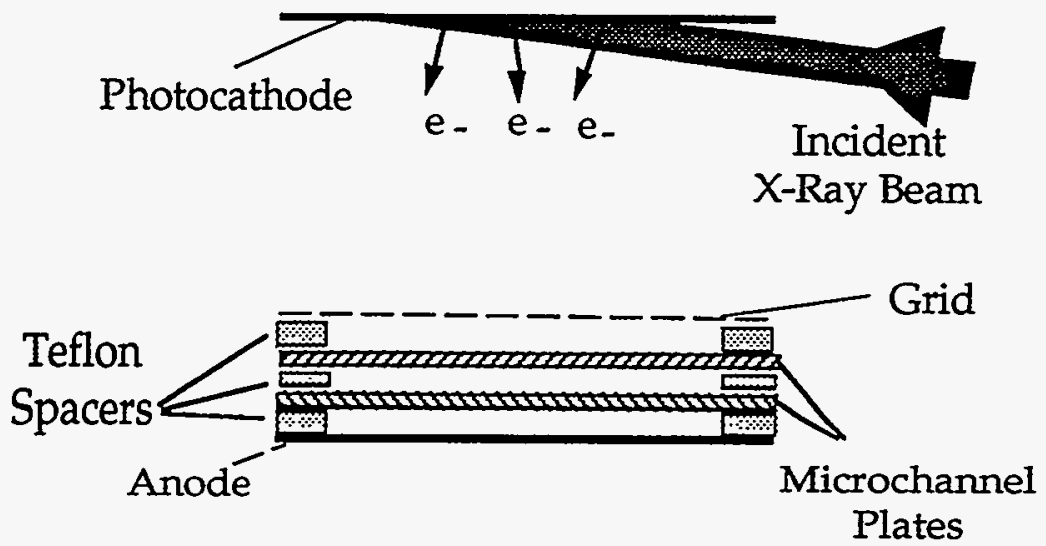

Figure 3.2. Schematic of the microchannel plate detector built at SSRL.

The vacuum chamber used for the $\mathrm{MCP}$ detector is shown in figure 3.3. The microchannel plate assembly was mounted on a 6" CONFLAT flange with 6 smaller (1.33") miniflanges to allow individual feed-throughs for the electrical connections (this "multiflange" unit was built by [UltraVac, 19]). This assembly was mounted in small stainless steel vacuum chamber based around a 4" diameter " $T$ ". The chamber was evacuated using a 55 liter/sec turbomolecular pump (Turbo-V60, [Varian Vacuum Products, 20]), backed by a roughing pump*. Two beryllium windows allowed the $x$-ray beam to pass into and out of the chamber (the second was for alignment purposes). The photocathode was placed on an XYZ stage with a rotary positioner [MDC, 21] that was motor

\footnotetext{
- The author discovered that ion pumps and microchannel plates do not mix well, due to the background from the ion pump. Inserting baffles reduced the background, but not sufficiently for this work.
} 


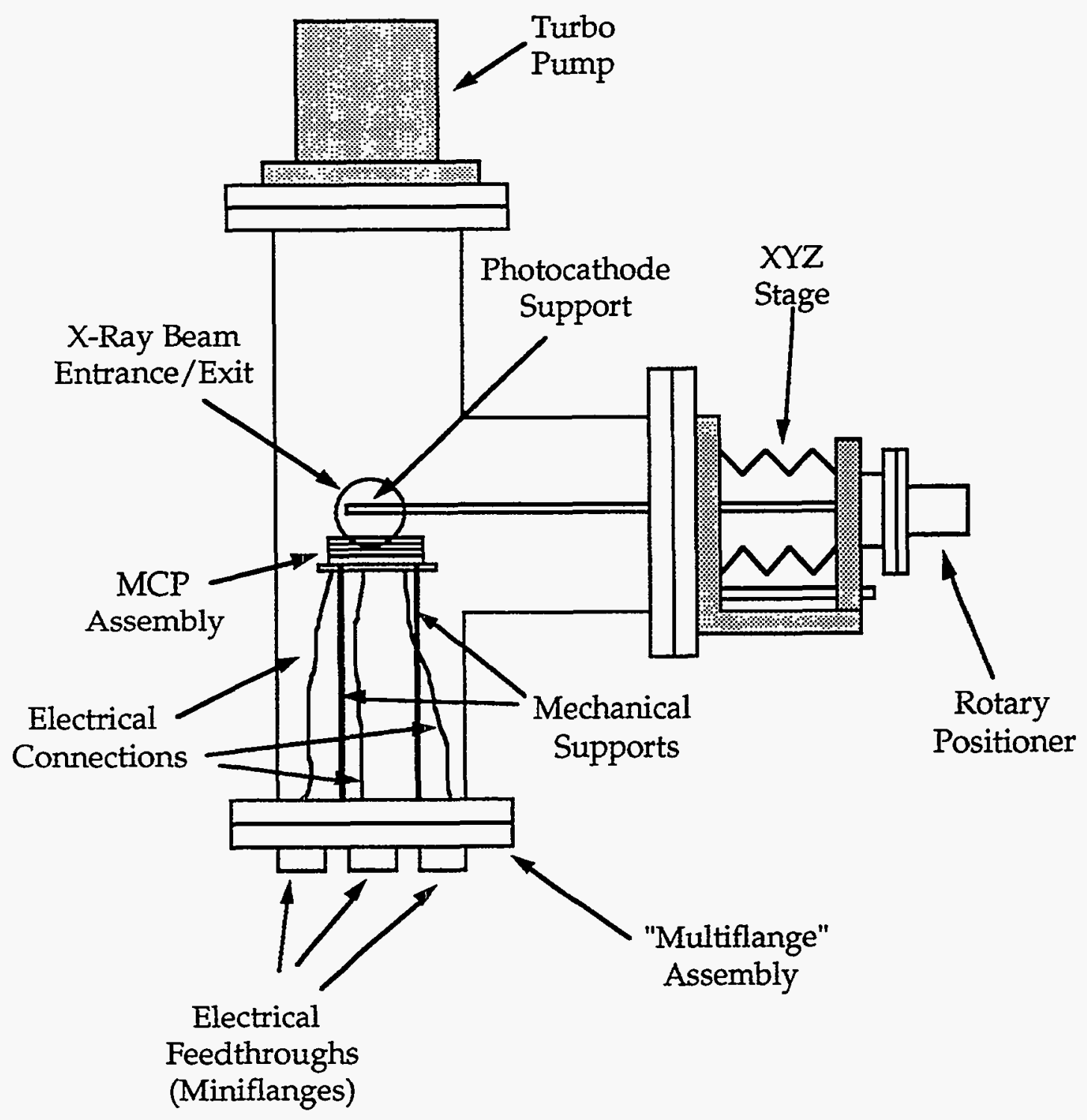

Figure 3.3. Schematic of the chamber used for the microchannel plate detector. Ports for a visible light window and an ion gauge are not shown. Figure is not to scale.

controlled with 0.05 degree precision. The high voltage pulses were coupled into the vacuum chamber using 0.25 " semi-rigid coaxial cable with an o-ring seal ("Swagelock" connector).

The pump down time of the chamber was typically a few hours to reach pressures of $<\sim 10^{-6}$ Torr, with a base pressure of a few $\times 10^{-7}$ Torr established within 24 hours. The detector was sometimes operated immediately (pressures 
of $\sim 10^{-6}$ Torr) but usually one waited until 24 hours had passed before putting high voltage on the microchannel plates. This was because the MCP's sometimes required 12 to 24 hours in vacuum before they would hold high voltage, after they had been exposed to room air. This was probably the result of moisture absorption.

\section{Gating the MCP Detector}

It was not possible to gate the detector using a simple grid operated at low voltage between the photocathode and the microchannel plates (as is done in the gated microchannel plate photomultiplier mentioned above). As is evident from figure 3.4, a substantial fraction of the time ( $20 \%$ at small grazing angles) absorption of a $14.4 \mathrm{keV}$ photon in the x-ray photocathode will lead to emission of a high energy ( $>\sim \mathrm{keV}$ ) electron. Effective gating via the grid would require a fast voltage pulse of several kilovolts (at least) which is difficult to produce, especially at $\mathrm{MHz}$ repetition rates.

Instead, gating was accomplished by changing the gain of the first MCP. This requires "only" a fast pulse of less than $1 \mathrm{kV}$ (possibly as low as 400V). Figure 3.5 is a schematic of the gating electronics for the microchannel plate (MCP). A pair of high voltage switches ("HV-1000 Pulsers", [Directed Energy Incorporated (DEI), 22]) were used to gate the microchannel plate (represented by a capacitive load in figure 3.5). These switches are based on a fast, high power MOSFET transistor and will drive up to 18 Amps into a $50 \mathrm{ohm}$ load with rise times of about $6 \mathrm{~ns}$ and (burst) repetition rates of $1 \mathrm{MHz}$. It was expected that with extra cooling, the switch could operate continuously at the $5 \mathrm{MHz}$ necessary for synchrotron radiation experiments ${ }^{*}$ with 200 ns between bunches.

The circuit of figure 3.5 resulted from the necessity of pumping a capacitive load ${ }^{+}$. The cables from each switch (with $R_{1}$ and $R_{2}$ ) were made short $\left(\sim 2^{\prime \prime}-6^{\prime \prime}\right)$ relative to the cable to the $M C P\left(\sim 4^{\prime}\right)$. The resistances $\left(R_{1}-R_{3}\right)$ were

\footnotetext{
${ }^{*} \mathrm{Up}$ to $3 \mathrm{MHz}$ would probably be possible with only adding extra cooling. To get to $5 \mathrm{MHz}$ would require rebuilding the switch driver circuit [Krausse (DEI), 1991, 23]

+A resistor could have been used in parallel with the $\mathrm{MCP}$, making it a well terminated resistive load, but this would require that $\sim 16 \mathrm{~kW}$ of power be put into the system and then dissipated (900 V into $50 \mathrm{Ohms}$ ) which was not considered practical.
} 


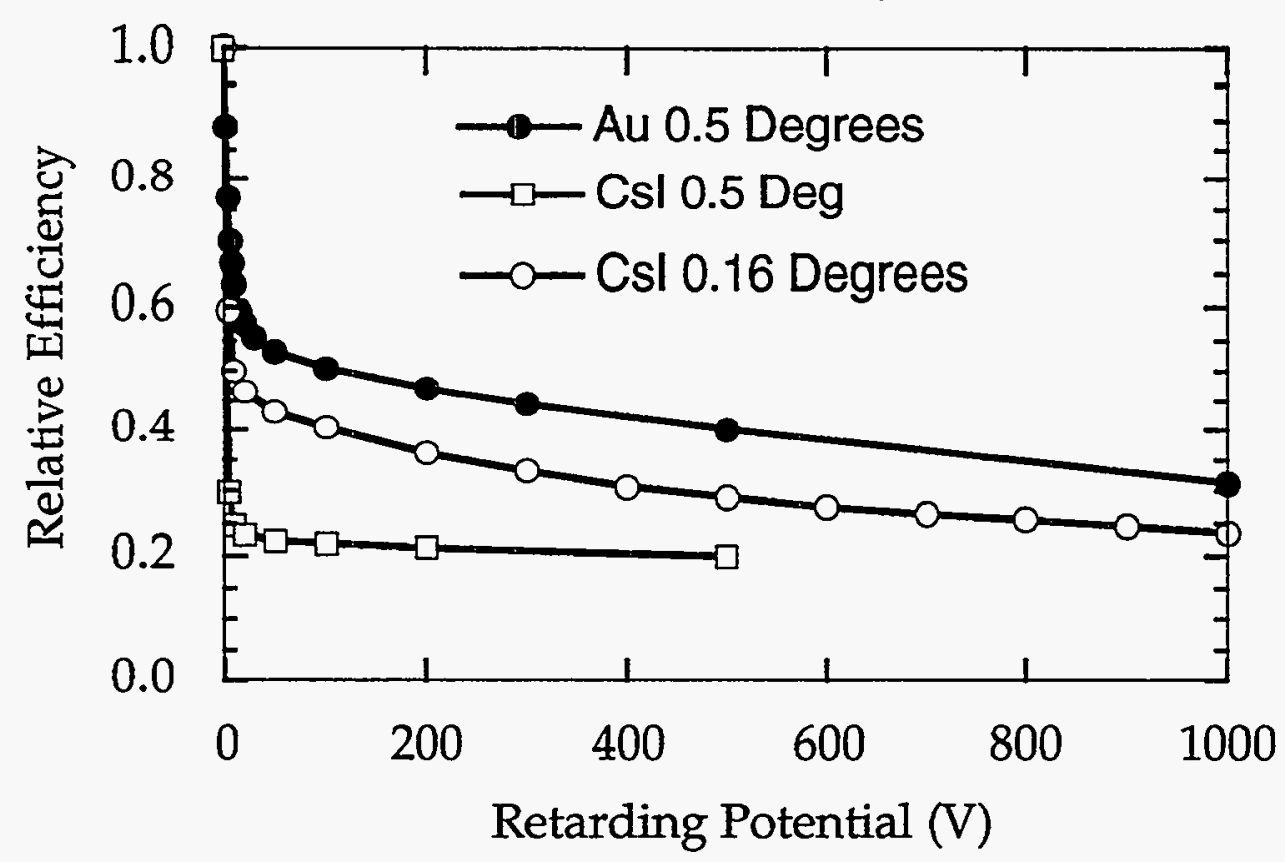

Figure 3.4. Effect of a retarding potential at the grid on the efficiency of the MCP detector. Measurement done with $14.4 \mathrm{keV}$ photons.

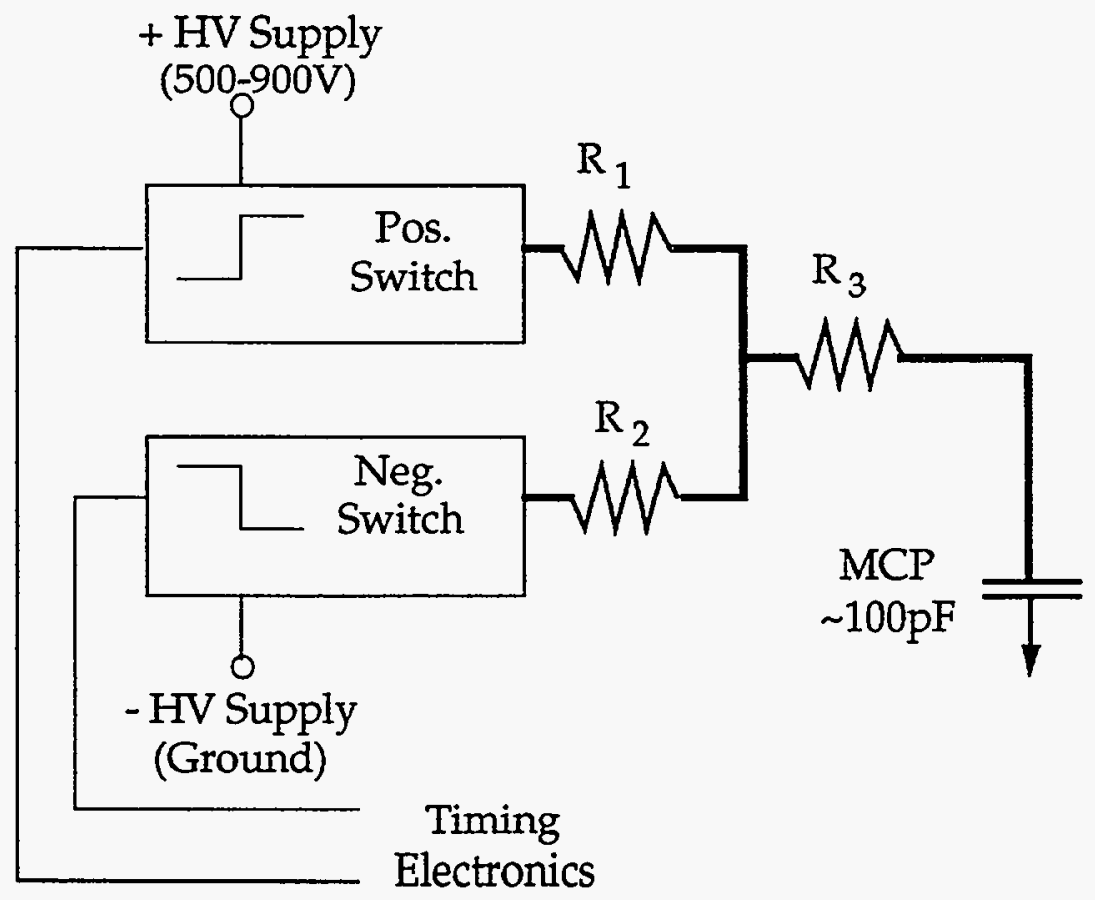

Figure 3.5. Schematic of the gating circuit for the MCP detector. Heavy solid lines represent $50-\mathrm{ohm}$ coaxial cable. 
chosen so that the pulse reflected from the capacitor would be terminated in 50 ohms (an open switch acted as open circuit). Thus, closing the positive (upper) switch when the negative (lower) switch was open resulted in (say) a 900V rising edge propagating from the switch through $R_{1}$ and $R_{3}$. The effect of the line to the open (lower) switch was to increase the rise time. Passing through $R_{1}$ and $R_{3}$, which total $50 \mathrm{ohms}$, reduces the voltage by half (to $450 \mathrm{~V}$ ) which then propagates down the line to the capacitive load of the MCP. There it is doubled by the reflection at the open circuit, leading to $900 \mathrm{~V}$ at the MCP. The wave reflected back from the MCP to the switches (an additional $450 \mathrm{~V}$ rising edge) is then terminated in $50 \mathrm{ohms}$ by $R_{1}$ and $R_{3}$. Practically, an asymmetric arrangement of resistances was usually chosen, $R_{1} \sim 4$ ohms, $R_{3} \sim 46$ ohms, and $R_{2} \sim 92$ ohms, which gave a good rising edge at the $\mathrm{MCP}$ ( $<10$ ns with little, $\sim 10 \%$, overshoot) and poor ( $50 \mathrm{~ns})$ falling edge.

\section{Results with the MCP Detector}

The MCP detector performed well on some basic levels. It was used to investigate some photocathode materials (see the next section) and was found to have an efficiency of about $50 \%$ at $14.4 \mathrm{keV}$ when used with a gold or CsI photocathode ( 0.5 degree angle of incidence of the radiation onto the photocathode). The time resolution, including the jitter in the electronics, was about $2.5 \mathrm{~ns}$, comparable with the plastic scintillation detector. Also, gating the first plate by reducing the voltage across it from 900 to $500 \mathrm{~V}$, was seen to reduce the count rate by more than 6 orders of magnitude (from $120 \mathrm{kcts} / \mathrm{sec}$ to $<0.1$ cts/sec).

However, when it became clear that the asymmetric nested monochromators (see chapter 2) were not too difficult to use, work on the MCP detector was abandoned in favor of developing an avalanche diode detector. The fancier monochromator design meant that the largest prompt rates would probably not be worse than 10 to 100 photons / pulse, thus making it unnecessary to gate the detector before any gain took place. The avalanche diodes seemed like a better (cheaper, easier) alternative to MCPs and, though not obvious at the time, it was hoped they could perform at moderately high count rates. 
It is worthwhile, however, to describe a few of the difficulties that it would have been necessary to deal with, if the work with the MCP detector had progressed further, particularly the (electronic) noise created by the MCP gating pulse and the dark current pulses from the microchannel plates.

Despite extremely careful shielding (essentially a Faraday cage was built around the anode), the ringing in the system after a $850 \mathrm{~V}$ gating pulse was of order 20 to $30 \mathrm{mV}$ immediately (in the first $30 \mathrm{~ns}$ ) after the gate and remained at the $10 \mathrm{mV}$ level out to several hundred $\mathrm{ns}$. This necessitated setting the discriminator much higher than without gating, and reduced the efficiency to rather less than $1 \%$ for single photons. In addition the time response was effected by the noise, becoming about 5 ns FWHM.

The dark noise count rates from the MCP's were typically $>\sim 100 \mathrm{~Hz}$ when no gating (and a low discriminator threshold) was used. This was not surprising given that the MCP's used in this work were not treated with great care (e.g. exposed to room air at the beamline). However, a good noise background rate for carefully handled MCP's is something like 0.1 to $0.5 \mathrm{cts} / \mathrm{sec} / \mathrm{cm}^{2}$ of surface area [Fraser, et al., 1986, 24] [Siegmund, et al., 1988, 16]. Thus for the $40 \mathrm{~mm}$ diameter plates used here one might expect 1 to $5 \mathrm{cts} / \mathrm{sec}$ of dark noise, assuming they had been treated well.

Finally, there were several hurdles to be overcome in going from the low, 10 to $20 \mathrm{kHz}$, gating rate used in most of the testing to the $5 \mathrm{MHz}$ rate necessary to do nuclear scattering experiments. In addition to rebuilding the drivers for the high voltage switches, it would be necessary to consider the performance of the entire system at much higher powers. This would probably have necessitated extreme care at all feed-throughs and connectors, as well as the use of high power resistors (e.g. those made by [KDI/Triangle Electronics, 25]).

\section{X-Ray Photocathodes}

One of the questions considered in conjunction with building the $\mathrm{MCP}$ detector was how to convert the incident $14.4 \mathrm{keV}$ x-ray into some sort of electron 
pulse for subsequent amplification: one reeded some sort of direct $x$-ray photocathode or perhaps a scintillator followed by a visible-light photocathode material. Direct detection of $\mathrm{x}$-rays using the microchannel plate (see [Bateman, 1977, 26] [Fraser, 1982, 27]) would have probably had an efficiency of less than $10 \%$, which was deemed insufficient at the time of this work. Scintillators were rejected due their relatively low efficiency and the presence of the slow light component (mentioned above) which might become a problem at high rates. One then needed to choose between different types of $x$-ray photocathodes.

The main concern in the choice of the photocathode material was the efficiency that could be achieved, though the ease of handling was also a consideration. The efficiency of a cathode is the result of trade-offs between the $x$-ray penetration into the cathode, the number of free electrons created by the $x$ ray and the possibility of those electrons reaching (and leaving) the surface of the material. See discussions in [Kane, 1966, 28] and [Henke, et al., 1979, 29].

A large number of photocathode materials have been experimentally investigated in the 1 to $8 \mathrm{keV} x$-ray region (see [Eliseenko, et al., 1968, 30] [Ebert, et al., 1969, 31] [Gaines and Hansen, 1976, 32] [Day, et al., 1981, 33] [Henke, et al., 1981, 34], and in particular, the paper by Henke, et al., is quite good). Based on this work, it was decided to investigate the properties of Au photocathodes and CsI photocathodes. The Au has a high $x$-ray stopping power (absorption length of $\sim 3 \mu \mathrm{m}$ at $14.4 \mathrm{keV}$ ) but low energy electrons tend not to travel far due to the electron-electron scattering in the conductor (electron attenuation length $\sim 30$ to $50 \AA$ ). The CsI has a lower stopping power (14.4 keV absorption length $\sim 35 \mu \mathrm{m}$ ) but the low energy electrons will also travel much further in the insulator (electron attenuation length $\sim 250-300 \AA$ ). It was expected a grazing incidence geometry would decrease the distance the electrons had to travel to leave the cathode, and thus increase the efficiency. Some more exotic photocathode materials, such as negative electron affinity (NEA) materials [Bell, 1973, 35] [Andrushchenko, et al., 1988, 36] and solid xenon [Gullikson and Henke, 1989, 37]

\footnotetext{
* Note that the this paper never specifies the angle of incidence of the photon beam onto the photocathode material. However, their results are only consistent with those of other authors if it is assumed that the photons are incident at a grazing angle of about 20 degrees, as was the case earlier work by the same authors.
} 
[Munier, et al., 1989, 38] were rejected since they introduced significant additional experimental difficulties.

Gold cathodes were made by evaporation of about $0.5 \mu \mathrm{m}$ of gold onto glass slides $\left(\sim 1 \times 3 \mathrm{~cm}^{2}\right)$ that had been cleaned and covered with a thin layer $(\sim 300 \AA)$ of $\mathrm{Cr}$. These were placed just above the MCP detector, as is shown in figure 3.2 and the efficiency of the detector was measured as a function of energy at several angles of incidence. The incident flux rate was determined by an $\mathrm{Ar}$ filled ion chamber calibrated against a $\mathrm{NaI}$ detector. Pulse rates were typically $\sim 10 \mathrm{kHz}$ or higher. Typical results are shown in figure 3.6. One notes that efficiencies approaching $50 \%$ at $14.4 \mathrm{keV}$ are possible at $~ 0.5$ degree angles of incidence. In addition, the measured efficiency shows improvement as each of the L-absorption edges $(11.9,13.7$ and $14.4 \mathrm{keV})$ are passed. This is due to the increased absorption cross section and corresponding reduced penetration of the $x$-rays into the material. The decrease in the efficiency at low energy and small grazing angles is due to total external reflection.

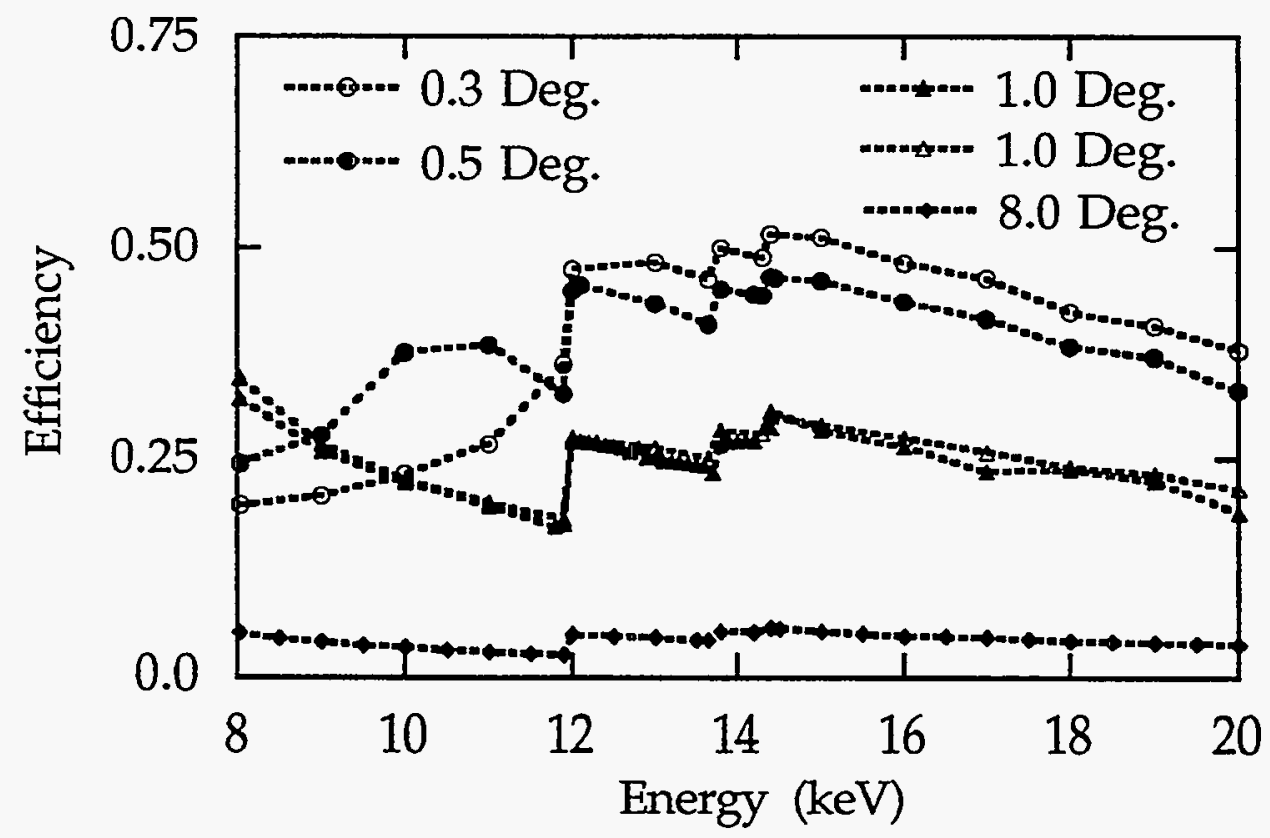

Figure 3.6. Efficiency of gold photocathodes at several grazing angles of incidence. Dashed lines are to guide the eye. Error bars are $\pm 10 \%$ on all points. Angles were determine to about \pm 0.07 degrees. 
Cesium iodide photocathodes were made by evaporation of about $1.0 \mu \mathrm{m}$ CsI onto $\mathrm{Cr}$ covered slides. CsI is slightly hygroscopic, so special precautions were taken to avoid exposing it to room air for extended periods of time (see [Whiteley, et al., 1984, 39],(Premaratne, et al., 1983, 40]) The total exposure time of the CsI photocathodes to room air was approximately 30 minutes.

Results from efficiency measurements using CsI photocathodes are shown in figure 3.7. At low energies the efficiency is much better than that of gold (due to the longer mean free path length of electrons in CsI). At higher energies, the efficiency drops off quickly due to increased penetration into the CsI. At 14.4 $\mathrm{keV}$, the CsI photocathode efficiency is actually slightly better than the Au efficiency at the same angle, but the difference is not too great ( $\sim 55 \%$ for CsI at 0.5 degrees, while Au gives $45 \%$ ).

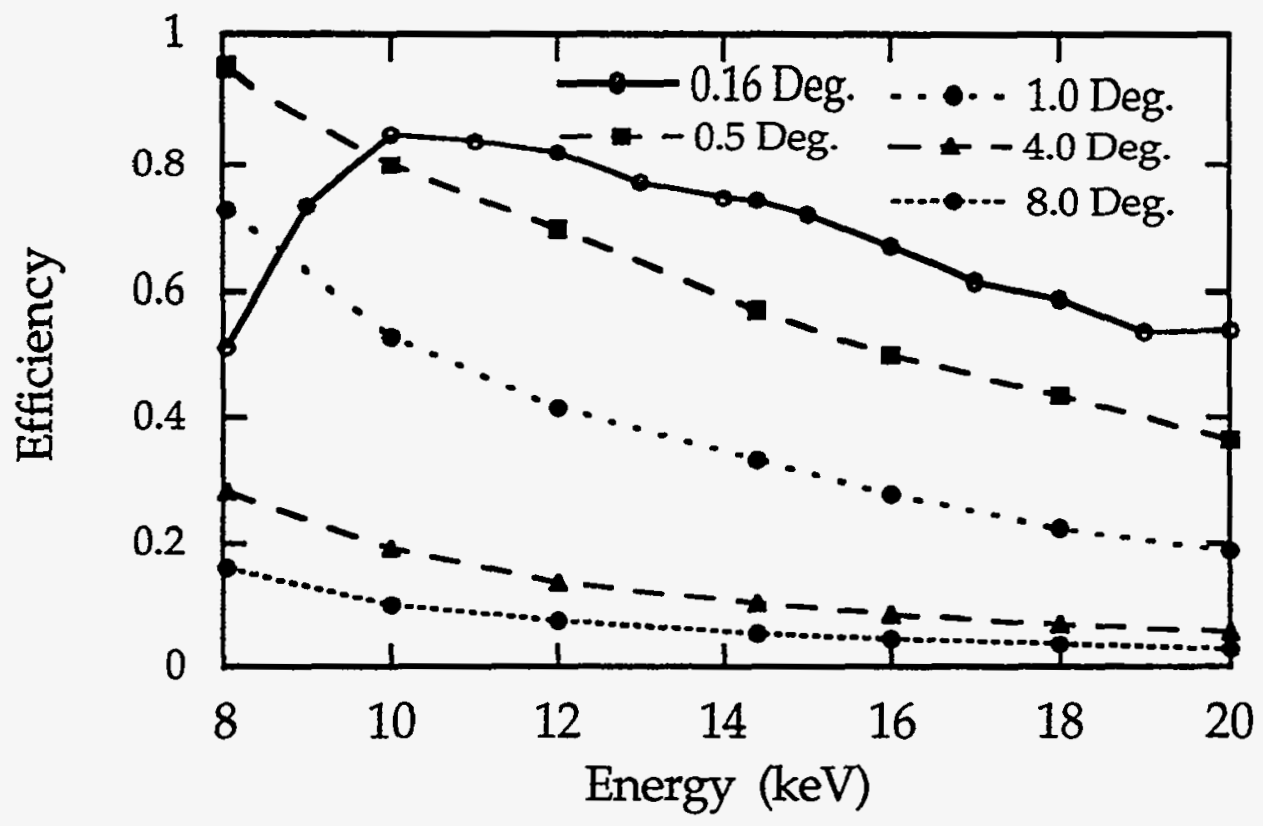

Figure 3.7. Efficiency of CsI photocathodes at several grazing angles of incidence. Lines are to guide the eye. Error bars are $\pm 10 \%$ on all points. Angles were determine to about \pm 0.07 degrees. 


\section{An Avalanche Photodiode Detector}

An avalanche diode is essentially a reversed biased p-n junction operated near its breakdown voltage. The electric fields are sufficiently high $\left(>\sim 10^{5}\right.$ $\mathrm{V} / \mathrm{cm}$ ) that photogenerated current will be amplified by impact ionization. Diodes may be operated above the breakdown voltage, in a "Geiger" mode [Lightstone, et al., 1989, 41], where a single carrier triggers run-away gain, or below breakdown, where there is a well defined average gain. See [Webb, et al., 1974, 42] and [Sze, 1981, 43] for reviews of avalanche diode operation. In this work, we will only be concerned with operation below breakdown, because operation above breakdown, while permitting very high sensitivity, also leads to high noise rates.

The use of avalanche diodes for $x$-ray detection dates back at least to the 1970's [Jones, 1973, 44][Webb and Jones, 1974, 45]. Their use as a timing detector in nuclear scattering experiments was pioneered by Kishimoto[Kishimoto, 1991, 46][Kishimoto, 1992, 47]. He showed that a $3 \mathrm{~mm}$ diameter diode (the S2384 made by [Hamamatsu, 6]) had a time resolution of $0.25 \mathrm{~ns}$ and an efficiency of $7.5 \%$ for $14.4 \mathrm{keV} x$-rays. However, the small diameter and low efficiency meant that it was not practical to use such a diode for resonant nuclear scattering experiments at SSRL, due to the large size of the x-ray beam (about $1 \times 10 \mathrm{~mm}^{2}$ ) and the low count rates of these experiments. Thus it was not until Ruby [Ruby, 1992,48 ] found that larger, $\left(>1 \mathrm{~cm}^{2}\right)$ devices were available [Advanced Photonix Inc., 49] that it became of interest to consider APDs for resonant nuclear scattering experiments at SSRL. In particular, this work investigates the $x$-ray time response of two varieties of large area APDs: beveled edge diodes made by API [Advanced Photonix Inc., 49], and reach through diodes made by EG\&G [EG\&G Optoelectronics Canada, 50]*.

Presently APD's are being used in a large number of applications, beyond the work described here. APD/Scintillator combinations are used to detect high energy ( $>\sim 100 \mathrm{keV}$ ) $x$-rays [Lecomte, et al., 1989, 52] [Carrier and Lecomte, 1990, 53] [Carrier and Locomte, 1990, 54] JJames, et al., 1992, 55]. Here advantages of APD's over PMTs include their immunity to magnetic fields (as high at 2 Tesla

\footnotetext{
* Another company making large area beveled edge diodes is RMD [Radiation Monitoring Devices, 51]
} 
[Raylman, 1993, 56]) and their small size and ruggedness. APDs may be used for direct detection of low energy ( $1.5 \mathrm{keV}$ [Webb and McIntyre, 1976, 57] [Farrell, et al., 1991, 58]) and very low energy $\mathrm{x}$-rays ( $50-300 \mathrm{eV}$ in gain mode [Gullikson, et al., 1994, 59] and 200-700 eV in Geiger mode [Palmer and Morrison, 1992, 60]). The time resolution of specially designed APDs has been pushed as low as $20 \mathrm{ps}$ (cooled and operated in Geiger mode [Cova, et al., 1989, 61]), while large area devices have shown time resolutions of $<100 \mathrm{ps}$ for high energy electrons [Hauger, et al., 1994, 62]. APDs have been used as the amplifying element in a photomultiplier tube [Cushman and Rusack, 1993, 63] [Szawlowski, et al., 1993, 64]: the photocathode is followed by a high field region that accelerates photoelectrons to about $10 \mathrm{keV}$ before they hit the surface of an APD, for a total gain of $10^{5}$ to $10^{6}$. Finally, multipixel arrays of APDs have been fabricated [Squillante, et al., 1986, 65] [Webb and Dion, 1991, 66] [Gramsch, et al., 1993, 67].

The theory describing the avalanche gain in these devices has been well developed [McIntyre, 1966, 68] [McIntyre, 1972, 69] [Webb, et al., 1974, 42]. The crucial parameter determining the statistics of the multiplication process is the average ratio of the gain for a hole to that for an electron. At the level of the work described here, however, it is sufficient to note that this ratio is small, (e.g. $\sim 0.002$ in the beveled edge devices [API, 70]) so that hole multiplication is ignored.

\section{APD Structure}

The $x$-ray response of the diodes may be very directly related to their structure. The structures of the two types of devices measured, the beveled edge and reach-through diodes, are two different responses to the same design problem. The gain in an avalanche diode depends sensitively on the local electric field strength, which is in turn a reflection of the doping profile. Thus the doping profile must be extremely uniform over the surface of the device or gain nonuniformities will arise, possibly leading to pre-mature breakdown as the voltage is increased.

The beveled edge design relies on neutron transmutation doped ( $\mathrm{Si}+\mathrm{n} \rightarrow>$ P) n-type wafers to achieve a very high uniformity [Gelezunas, et al., 1977, 71]. 
Gallium diffusion creates a p-type region and a junction that is typically $\sim 40 \mu \mathrm{m}$ from the surface (see figure 3.9) When high voltage (2200-2500V) is applied to the beveled edge device the depletion region spreads out from the $p-n$ junction to

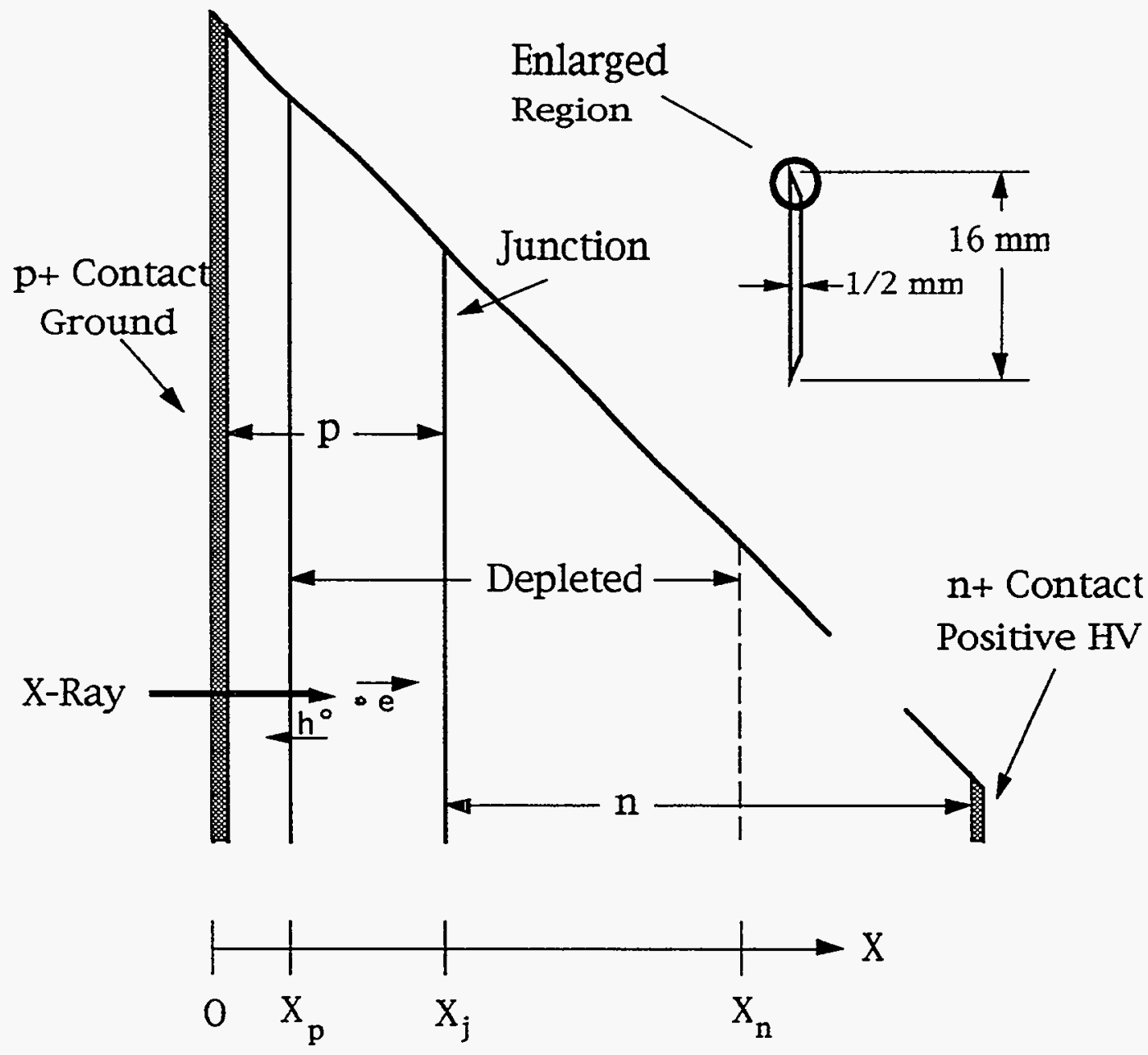

Figure 3.8. Beveled edge APD structure. $X$-rays enter through a thin $(\sim 0.2 \mu \mathrm{m}) \mathrm{p}+$ window at the device surface $(x=0)$. The depletion region extends from $x_{p}$ in the p-region to $x_{n}$ in the $n-$ region. A thin $(\sim 0.1 \mu \mathrm{m})$ passivation layer is not shown.

within 5 to $10 \mu \mathrm{m}$ of the surface and deep into the $\mathrm{n}$ region, resulting in the field profile shown in figure $3.9 \mathrm{~b}$. The front (undepleted) portion of the p-region has a residual field estimated to be $\sim 50 \mathrm{~V} / \mathrm{cm}$ [API, 72], resulting from the high doping gradient. The field near the junction is estimated to be $\sim 1.8 \times 10^{5} \mathrm{~V} / \mathrm{cm}$, sufficient to cause impact ionization by electrons traversing the region. Figure $2 \mathrm{c}$ shows the approximate distribution of the ionization coefficient (the inverse of 
the average distance an electron must travel to generate an electron-hole pair). This is effectively the local electron gain of the device. Note that it is spread out over a fairly large (many $\mu \mathrm{m}$ ) distance. The total gain for an electron entering this region are can be quite large, approaching $10^{3}$ [Szawlowski, et al., 1992, 73] (or even higher [Farrell, et al., 1994, 74]).

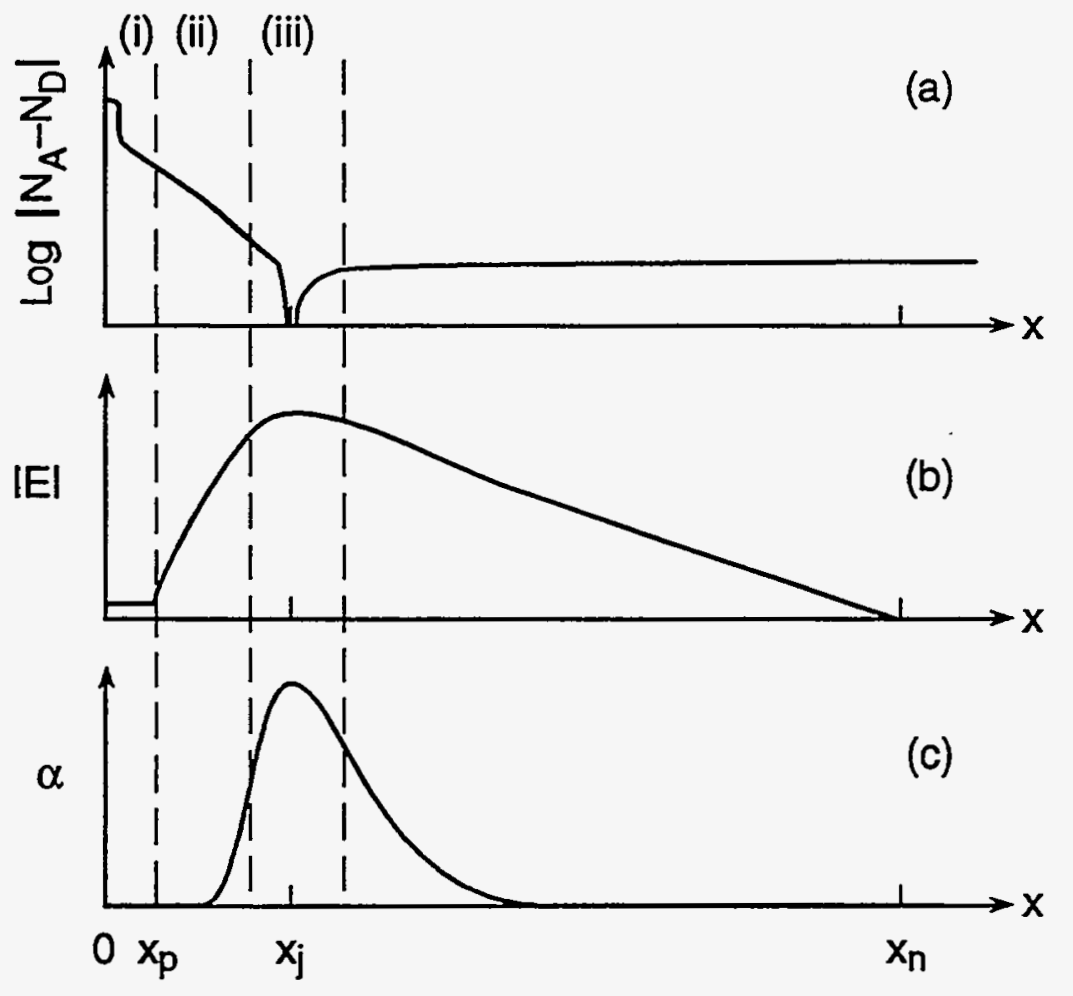

Figure 3.9. Approximate (a) doping, (b) field and (c) ionization coefficient (local gain) for the API beveled edge diodes. The vertical dashed lines are approximate divisions for the regions discussed in the text. The figure has been adapted from one in [API, 72], courtesy of API.

The reach though device is based on lightly doped $\pi$-type wafers, and the structure is shown in figure 3.10. A junction is made near to one surface and when high voltage is applied, the depletion region "reaches through" to the $\pi$ region, eventually reaching all the way to the $\mathrm{p}+$ contact (see [Webb, et al., 1974, 42]). Figure 3.11 shows the approximate doping concentrations and field profile. Gain only takes place in a small (few $\mu \mathrm{m}$ ) region in the vicinity of the peak of the 


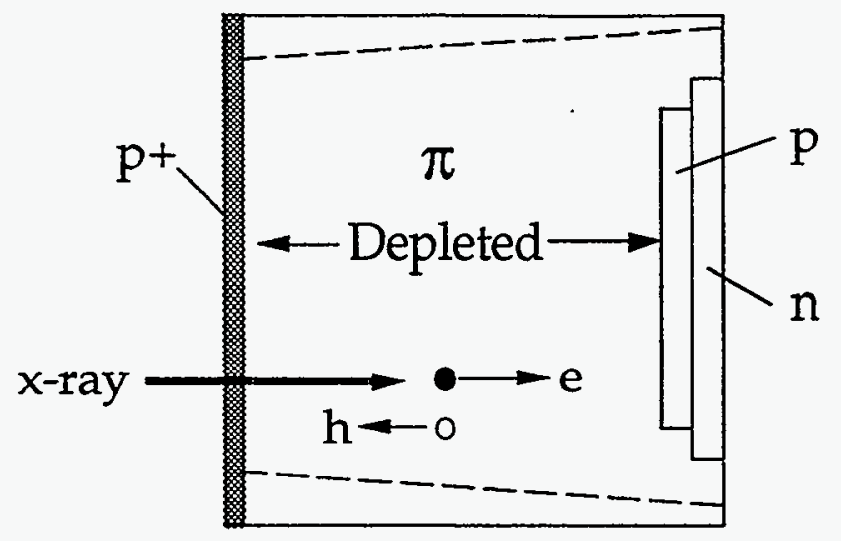

Figure 3.10. Schematic of a reach through diode. The total thickness is $\sim 100 \mu \mathrm{m}$. Figure based on one in [Webb and Jones, 1974, 45] and information from [EG\&G Optoelectronics Canada, 50]. A passivation layer and a guard ring at the $n$ surface are not shown.
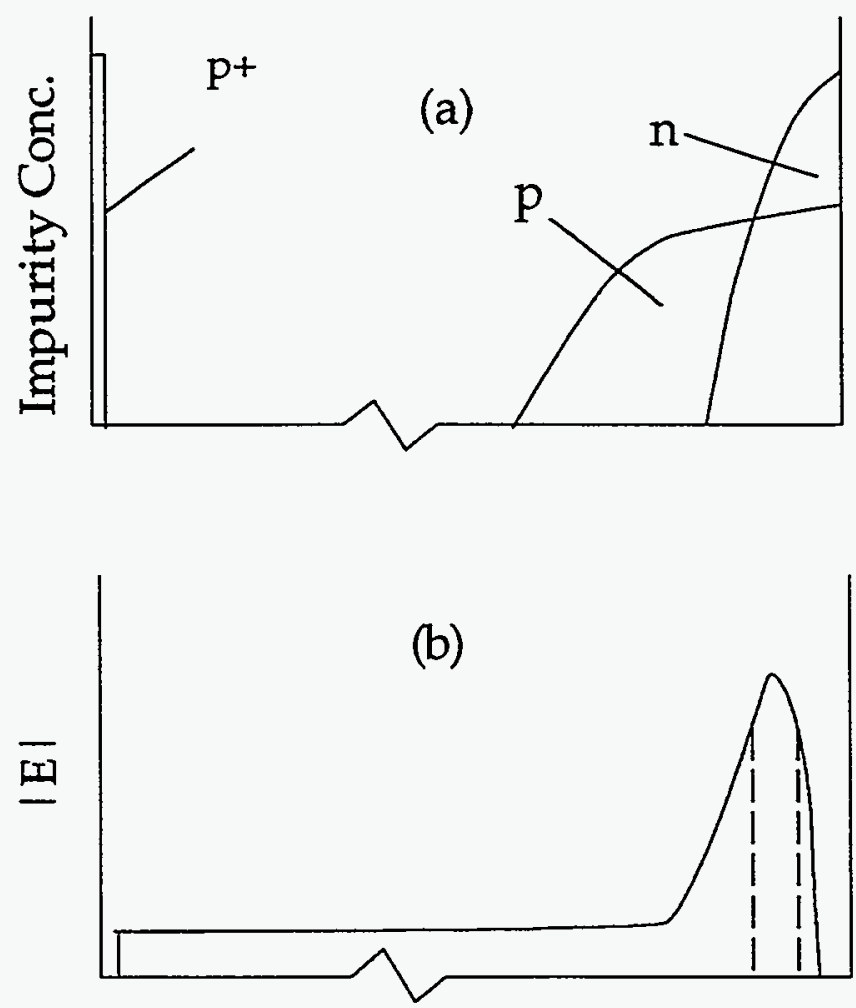

Figure 3.11. (a) doping and (b) field profiles for a reach through diode. The dashed lines in (b) are meant to show the approximate boundary of the gain region. Figure based on one in [Webb and Jones, 1974, 45]. 
electric field. The bulk of the material has a field high enough to nearly saturate the electron drift velocity, but not sufficiently high to cause gain.

\section{Model for X-ray Response}

X-rays incident from the $\mathrm{p}+$ side of a diode will be absorbed in the silicon with a characteristic $(1 / e)$ attenuation length $\mathrm{L}_{\mathrm{abs}}\left(\mathrm{E}_{\gamma}\right)$, where $\mathrm{E}_{\gamma}$ is the photon energy. This is plotted in figure 3.12 [McMaster, et al., 1969, 75]. The majority of absorption events result in the production of a fast electron having nearly the energy of the incident photon. This fast electron slows, generating one electronhole pair for every 3.6 eV of energy [Ryan, 1973, 76], over some characteristic distance: the range for a $10 \mathrm{keV}$ electron in $\mathrm{Si}$ is approximately $1.4 \mu \mathrm{m}$ [Ashley, et al., 1976, 77] . On the scale of field variations within the diode, each $x$-ray absorption event may be considered a point like deposition of $E_{\gamma} / 3.6 \mathrm{eV}$ electronhole pairs.

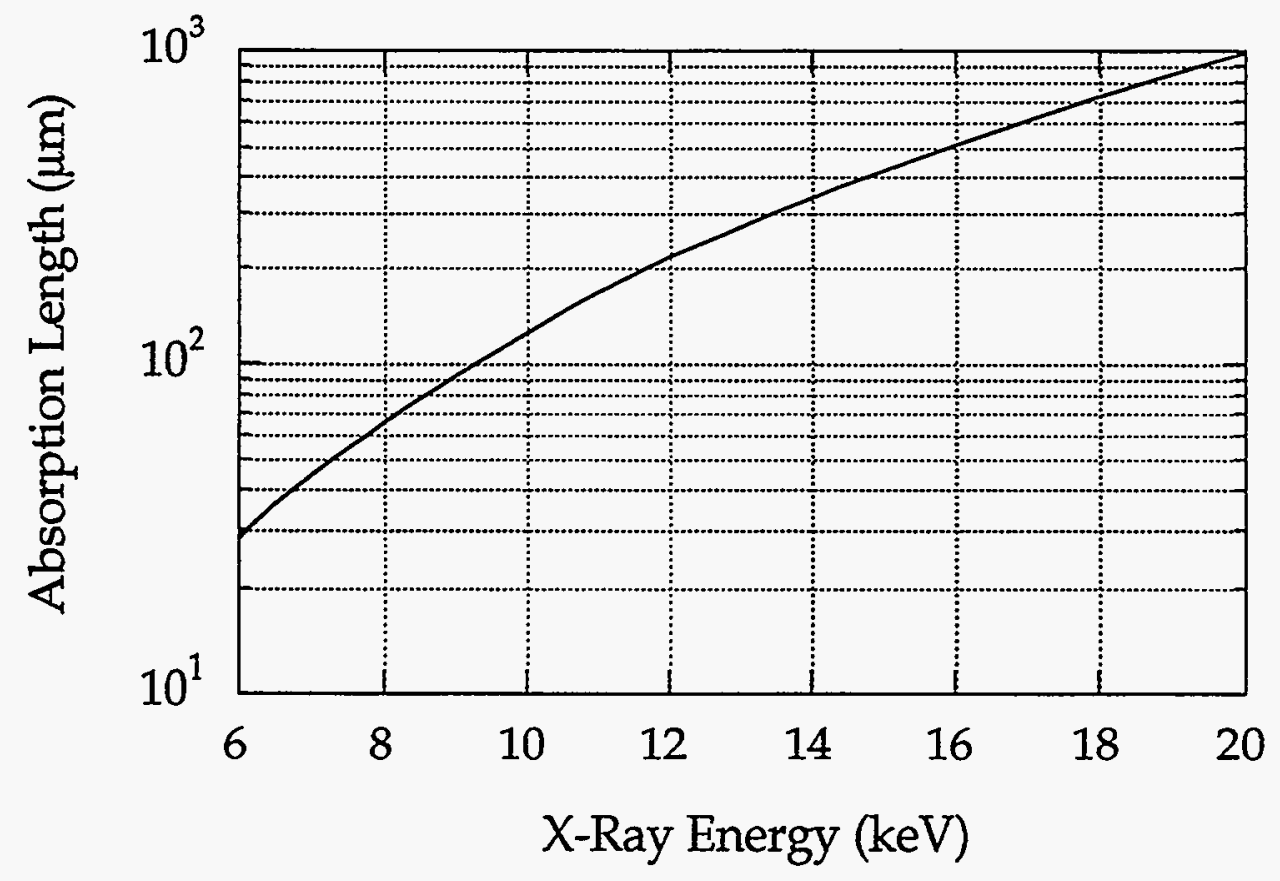

Figure 3.12. Absorption (1/e) length for $\mathrm{x}$-rays in silicon. From fits in [McMaster, $1969 \# 167$ ]

\footnotetext{
* The $1.4 \mu \mathrm{m}$ range is from a continuous slowing down approximation (CSDA) from stopping power tables. An empirical range-energy relation in [Katz and Penfold, 1952, 78] gives a $0.7 \mu \mathrm{m}$ range for $10 \mathrm{keV}$ electrons in $\mathrm{Al}$ of density $2.3 \mathrm{~g} / \mathrm{cc}$.
} 
The single photon, $x$-ray response of these APDs can be understood by considering the locations at which the $\mathrm{x}$-rays are absorbed. For the beveled edge structure, there are three regions of interest (see figures 3.8 and 3.9).

(i) X-rays absorbed in the undepleted p-region (the first 5 to $10 \mu \mathrm{m}$ of the device), where there is a small electric field, will generate electrons that will be slowly $(1.3 \mathrm{~ns} / \mu \mathrm{m}$ for a field of 50 $\mathrm{V} / \mathrm{cm}$ in pure Si [Jacoboni, et al., 1977, 79]) transported to the edge of the high field region, where they will then be swiftly carried to the high gain region and amplified. However, there will be a delay of as much as several ns due to the time required to traverse the low field region. In addition, traps [Miller, et al., 1977, 80] in this region may hold electrons for long periods of time (10s of ns to $\mu \mathrm{s}$ [Cova, et al., 1989, 61]) causing a reduction in the peak amplitude of the current pulse getting to the high field area, as well as lengthening its trailing edge as the electrons are released. These effects combine to give output pulses from the APD having lower peak height, a slower fall time and, possibly, a slower rise time than pulses from absorption of $x$-rays in the high field region ((ii), below). The pulses from this region may also have a lower integrated charge if some of the traps lead to recombination, as well as delay.

(ii) X-rays absorbed in the front portion of the depleted $\mathrm{p}$ region will generate pulses with a fast rise time and the complete gain of the device. Electrons will be quickly $(<1 \mathrm{~ns})$ transported to the high gain region of the device and be amplified with approximately the average device gain, $\mathrm{M}$.

(iii) $\mathrm{X}$-rays absorbed within the gain region of the device, either at the back of the depleted $p$ region, or in the front of the depleted $\mathrm{n}$ region, will generate electrons that will only be partially amplified, resulting in lower amplitude pulses that will appear (up to a scale factor) much like pulses from region (ii) above. The amplitude of these pulses should vary continuously to zero. 
The structure of the EG\&G diode is somewhat simpler, and the $x$-ray response is also somewhat simpler. In particular, the bulk of the reach-through diode behaves as region (ii) of the beveled edge device. There is no evidence, in the $x$-ray response, for a low field region at the front of the diode, and the gain region at the back of the device is smaller, so that it does not play a large part in the $x$-ray response.

\section{Experimental Conditions: X-ray Source}

The measurements described below were all done at beamline 2-3 of SPEAR at SSRL. A two crystal symmetric Bragg reflection monochromator selected a narrow bandwidth $(<0.1 \%)$ of radiation. A slight angular shift between the two crystals was introduced to reduce the contribution of higher order harmonics. The photon beam was collimated to a small spot $(\sim 50 \times 100$ $\mu \mathrm{m}^{2}$ ) and passed through an ion chamber before falling on the diode.

The time structure of the $\mathrm{x}$-ray pulses is the same as that for the electron bunches in the storage ring (SPEAR). The rf (radio-frequency) of the accelerating field, $358 \mathrm{MHz}$, confines electrons to bunches of nearly Gaussian shape and small width (full width at half maximum, FWHM, $0.13 \mathrm{~ns}$ [Nuhn, 1992, 81]) separated by integer multiples of $2.8 \mathrm{~ns}=1 / 358 \mathrm{MHz}$. Typically, only every fourth or fifth rf bucket was filled with electrons, around $\sim 2 / 3$ of the ring circumference, with $\sim 1 / 3$ of the circumference left empty. The electron distribution is then a series of bunches, separated by 11.2 or $14 \mathrm{~ns}$, followed by a dead time of $\sim 250 \mathrm{~ns}$. This was repeated at $1.28 \mathrm{MHz}$, or every $780 \mathrm{ns,} \mathrm{corresponding} \mathrm{to} \mathrm{the} \mathrm{ring}$ circumference. Upon close examination, smaller bunches (by a factor of $10^{3}$ ) were discovered between the bunches described above. These "minibunches" were an irritant in the measurements described below, but not a serious problem, since they could be identified by their $2.8 \mathrm{~ns}$ periodicity (figure 3.19).

\section{Electronics}

Figure 3.13 is a schematic of the electronics used in these measurements. The signal was capacitively coupled from the HV side of the diode (this was necessitated by the design of the API diodes, but might be avoided with the 
EG\&G diodes). The coupling capacitor was initially chosen to be $100 \mathrm{pF}$, but this leads to a reduction of the signal height, so later a $4700 \mathrm{pF}$ capacitor was used. The protection resistor was typically $\sim 10 \mathrm{meg}$, but for high rate experiments was sometimes reduced to $1 \mathrm{meg}$. This prevents the voltage drop across the resistor from significantly reducing that across the diode and reducing the gain. Finally, for the EG\&G diode, an additional lead from the HV to the guard ring was used.

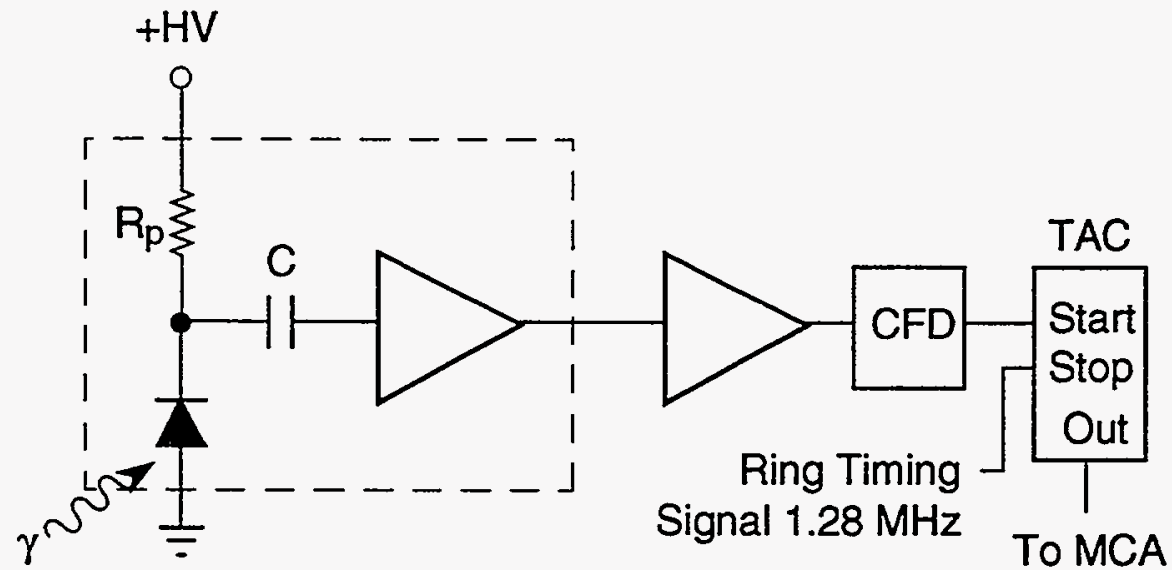

Figure 3.13. Schematic of the electronics for the APD detectors.

The APD was kept at positive high bias using a Bertan current limited voltage supply. The limit was set so that if the APD drew more than $8 \mu \mathrm{A}$ current, the high voltage would shut off. This happened perhaps 5 times during a 10 day experimentation period. Only once did the supply shut off at APD gains of less than $\mathrm{M} \sim 500$ (most shut offs were at gains of 1000, or higher, and therefore, at higher voltages as well). Subsequent to the measurements presented here, it was found that the shut offs could have been the result of poor HV insulation: silicon RTV works nicely, electrical tape should be avoided.

The signal from the APD was immediately fed into a Phillips 6954 pulse pre-amplifier (x100) [Phillips, et al., 1989, 82]. These are wide-band amplifiers nominally having an input noise of less than $30 \mu \mathrm{V}$, RMS over a $100 \mathrm{kHz}-1.8$ $\mathrm{GHz}$ bandwidth* . Measurement of the output noise using a digital scope showed it to be about 2 to $2.5 \mathrm{mV}$ in a $500 \mathrm{MHz}$ bandwidth, corresponding to 20 to $25 \mu \mathrm{V}$

\footnotetext{
* Amusingly enough, this is a little better than what one would expect from the Johnson noise of a $50 \mathrm{ohm}$ resistor which is given by $\mathrm{V}_{\mathrm{rms}}=(4 \mathrm{kTRB})^{1 / 2}=1.27 \times 10-4 \mathrm{R}^{1 / 2} \mu \mathrm{V} / \mathrm{Hz}^{1 / 2}=37 \mu \mathrm{V}$ for 50 ohms and $1.7 \mathrm{GHz}$. See [Horowitz and Hill, 1980, 83] p. 288.
} 
at the input (it did not matter if the input was terminated in $50 \mathrm{ohms}$, or allowed to float). Recently, we have also used a pair of Mini-Circuits MAR-6 amplifiers [Mini-Circuits, 84] in place of the Phillips 6954. The noise from these amplifiers is about 15-20 $\mu \mathrm{V} \mathrm{RMS}$ at the input ( $500 \mathrm{MHz}$ bandwidth) and, in addition, the MAR-6's are cheaper ( $\sim \$ 10$ vs. $\sim \$ 300$ for the 6954 ). The MAR- 6 's do require a small amount of circuit design but they can also be positioned very close to the diodes and seem to improve the signal to noise ratio by about a factor of two, relative to the Phillips amplifiers. Figure 3.14 shows the output from both the API and EG\&G diodes for $14.4 \mathrm{keV} x$-rays after amplification by the 6954 preamp and averaged over many events.

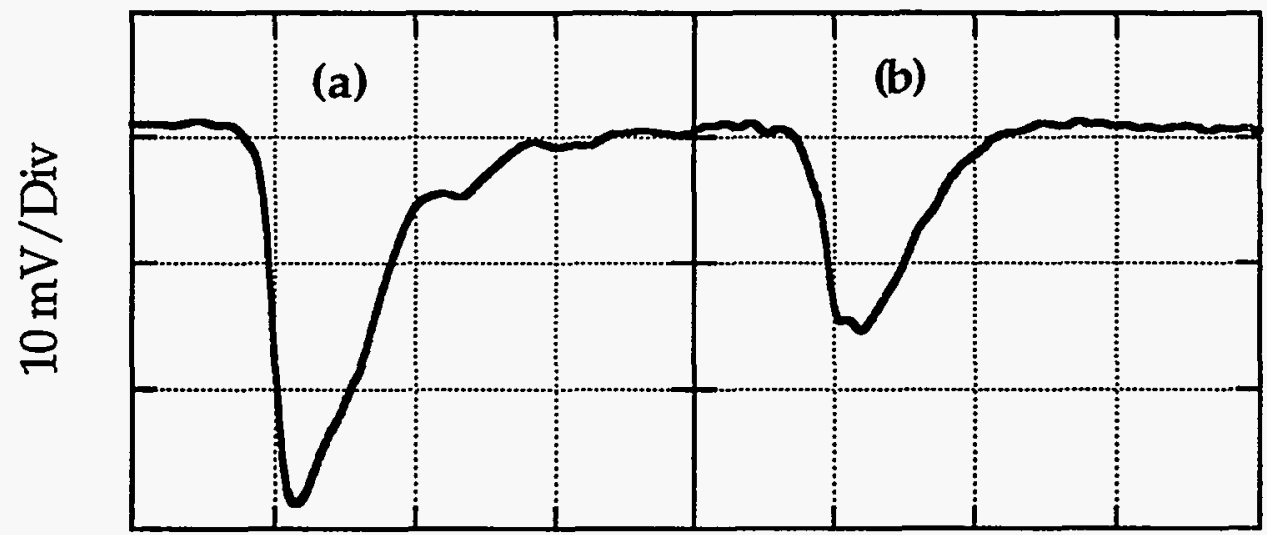

10 ns/Div

$10 \mathrm{~ns} / \mathrm{Div}$

Figure 3.14. Diode response to $14.4 \mathrm{keV} x$-rays, averaged over many events. (a) is for an API beveled edge diode at gain about 300. The fall time is $1.8 \mathrm{~ns}$, the rise $13.3 \mathrm{~ns}$ for a width of $7.6 \mathrm{~ns}$. (b) is for an EG\&G reach through diode with gain about 150 . The fall time is $2.4 \mathrm{~ns}$, the rise about $7.3 \mathrm{~ns}$ and the width about $7.0 \mathrm{~ns}$.

Additional amplification was necessary to reach the minimum threshold of the discriminators. This was provided by either a EG\&G FTA 420 amplifier ${ }^{+}$ $(x 20,20-300 \mathrm{MHz})$ [EG\&G Ortec, 85] or a LeCroy 612A amplifier (x10, DC-200 $\mathrm{MHz}$ ) [LeCroy, 86]. It is worth noting that the reduction in bandwidth of these

+ In some recent work at high rates ( $>10^{6} x$-rays $/ \mathrm{sec}$ ) it was noticed that the EG\&G FTA 420 amplifiers had the disturbing property that they seemed to amplify large pulses more effectively than small ones. No clear explanation has presented itself yet but the evidence from both the counting rates and the scope traces was very clear (it did not seem to be due only to a baseline shift). 
amplifiers is a boon, not a problem, since the bandwidth of the signal is less than $200 \mathrm{MHz}$ (see figure 3.12). More careful design of the pre-amplifiers (e.g. replacing the 6954 or MAR-6 described above) would limit would limit the bandwidth of these as well, probably to the level of $100 \mathrm{MHz}$ [Hauger, et al., 1994, 62].

The time response of the diodes was determined by measuring the interval between output of a constant fraction discriminator (EG\&G Ortec 934 CFD) and a signal synchronized to the $1.28 \mathrm{MHz}$ ring frequency of the synchrotron (and therefore locked to the time at which photons hit the APD surface). The CFD output was used as a start to a time to amplitude converter (TAC) while the $1.28 \mathrm{MHz}$ signal was used as a stop. A check of the ring timing signal against a delayed copy of itself showed the jitter in the electronics to be $\sim 0.2$ ns.

\section{X-Ray Efficiency}

The x-ray efficiency of these diodes can be modeled by assuming that all photons absorbed in some active thickness ( $\mathrm{L}_{\mathrm{act}}$ ) of silicon are detected. The efficiency as a function of energy should then be given by

$$
E\left(E_{\gamma}\right)=c\left(E_{\gamma}\right)\left[1-e^{-L_{a c t} / L_{a b s}\left(E_{\gamma}\right)}\right]
$$

where $L_{a b s}\left(E_{\gamma}\right)$ is the absorption length in silicon for x-rays of energy $E_{\gamma}$. The term in brackets is simply the number of x-rays absorbed the active thickness of silicon (passivation and $p+$ layers at the surface are ignored). The term $c\left(E_{\gamma}\right)$ is a correction factor to compensate for the fact that not all absorption events generate electrons. It is the ratio of the photo-electric cross section to the total cross section (photo-electric+Compton+Rayleigh) and varies from 0.99 at $8 \mathrm{keV}$ to 0.93 at $20 \mathrm{keV}$ [McMaster, et al., 1969, 75].

In practice, the gain variations from event to event and the finite discriminator threshold mean that the measured efficiency may not exactly follow the form of (3.1), but if one scales the discriminator threshold with the 
energy of the incident photons, the agreement is actually quite good. Thus, figure 3.15 shows the measured values and the fits based on equation 3.1 for two beveled edge diodes from API and one reach through diode from EG\&G. As expected, the EG\&G device has a much higher efficiency, corresponding to more than $90 \mu \mathrm{m}$ active thickness of $\mathrm{Si}$ while the API devices have active thicknesses of more like 40 to $50 \mu \mathrm{m}$ of silicon. In fact, the majority of the API diodes have efficiencies corresponding to about $40 \mu \mathrm{m}$ of silicon, and the higher value, about $50 \mu \mathrm{m}$ was only observed for one device that went to very high gains ( $M \sim 2500$ ).

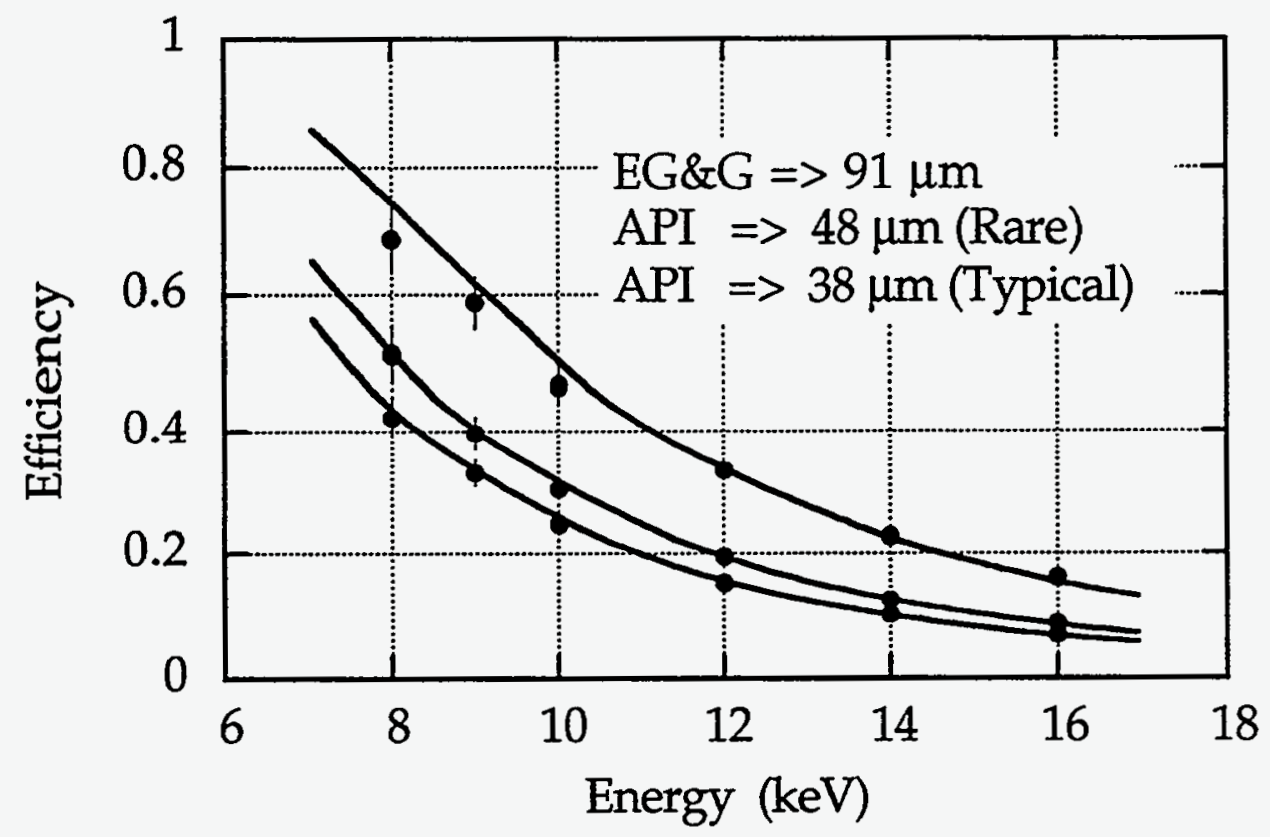

Figure 3.15. Measured APD efficiency and fit using equation 3.1. Active thicknesses are as listed, and correspond to the traces from top to bottom.

\section{Time Response}

The time response of the two types of diodes is shown in figure 3.16, on both linear and logarithmic scales. The response of the beveled edge diode has a narrow peak of about $0.5 \mathrm{~ns}$ FWHM and a tail out to longer times while the reach through device has a slightly larger FWHM, but no tail at longer times. 
The long tail from the beveled edge device results from the low field undepleted region at the front of the device. This is confirmed both by the time delay and by the variation of the size of the tail with the energy of the incident photons. Figure 3.17 shows how the tail size increases at lower energies, which is what one would expect given the x-ray absorption length becomes smaller. In fact for the high gain APD mentioned above the fraction of events occurring $1 / 2$ ns after the peak was measured as a function of energy. The efficiency of this slow region is well fit by the equation (3.1), as is evident from figure 3.18, giving the thickness of the low field region to be about $9 \mu \mathrm{m}$. Taking the tail length to be about $4 \mathrm{~ns}$, gives the average velocity in the low field region to be about 2.3 $\mu \mathrm{m} / \mathrm{ns}$, corresponding to an average field of about $160 \mathrm{~V} / \mathrm{cm}$ (mobility $\sim 1450$ $\mathrm{cm}^{2} / \mathrm{s} / \mathrm{V}$ [Jacoboni, et al., 1977, 79]) which is somewhat different than the 50 $\mathrm{V} / \mathrm{cm}$ value suggested by API [API, 72]. The reason for this difference is not understood. 


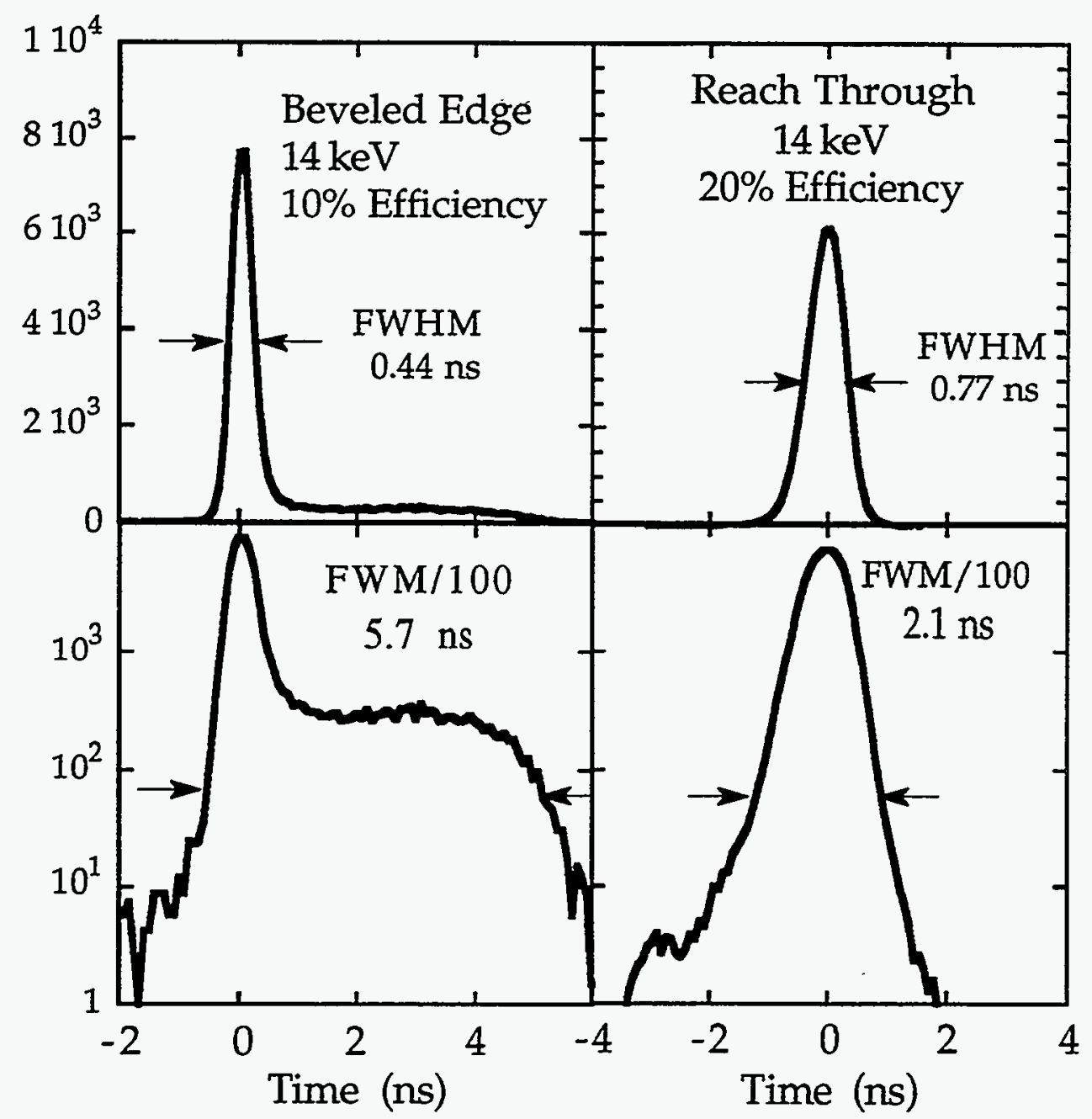

Figure 3.16. Time response to $14 \mathrm{keV} x$-rays for beveled-edge and reach through diodes. This may be interpreted as the probability distribution for the time between when an $x$-ray enters the APD and when the when an output pulse above the discriminator threshold is generated. The zero time has been arbitrarily set at the peak position and the values for the FWHM and full width at 100th maximum are as shown. 


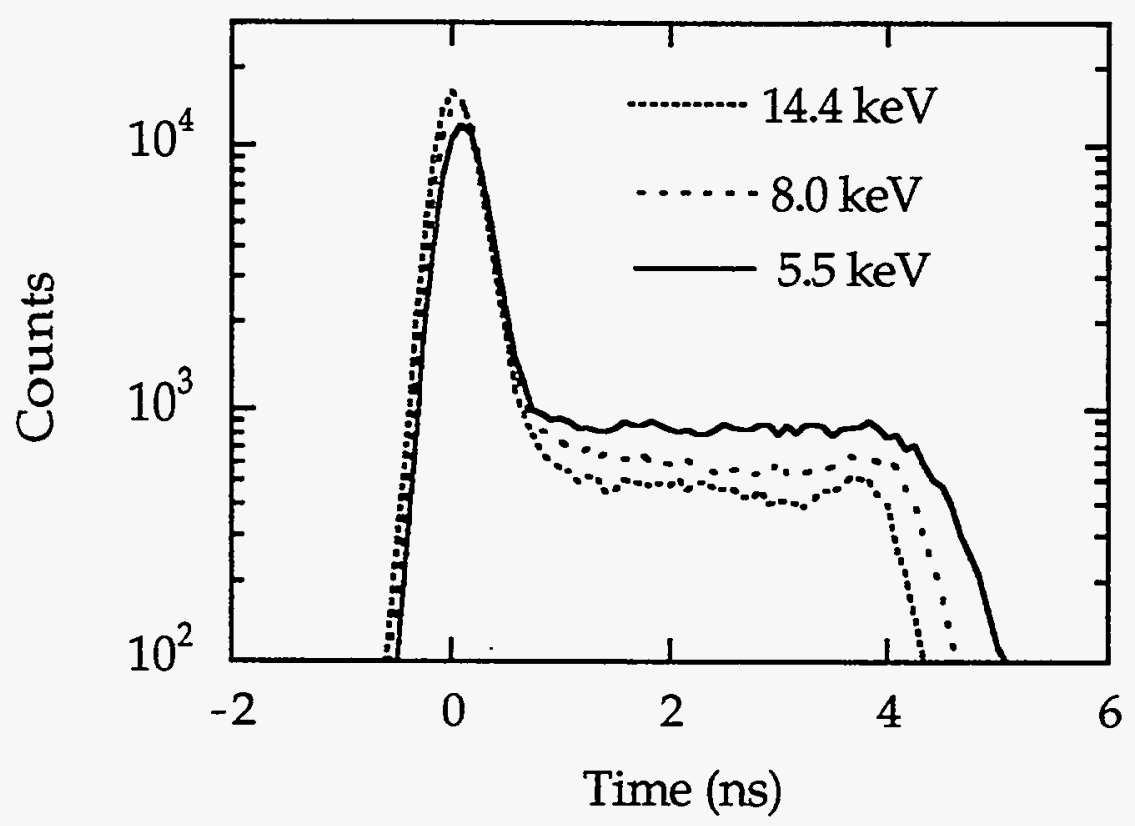

Figure 3.17. Variation in time response of a beveled edge APD with $x$-ray energy. The fraction of events in the tail increases as the energy of the incident photons drops and the $x$-ray absorption length becomes smaller.

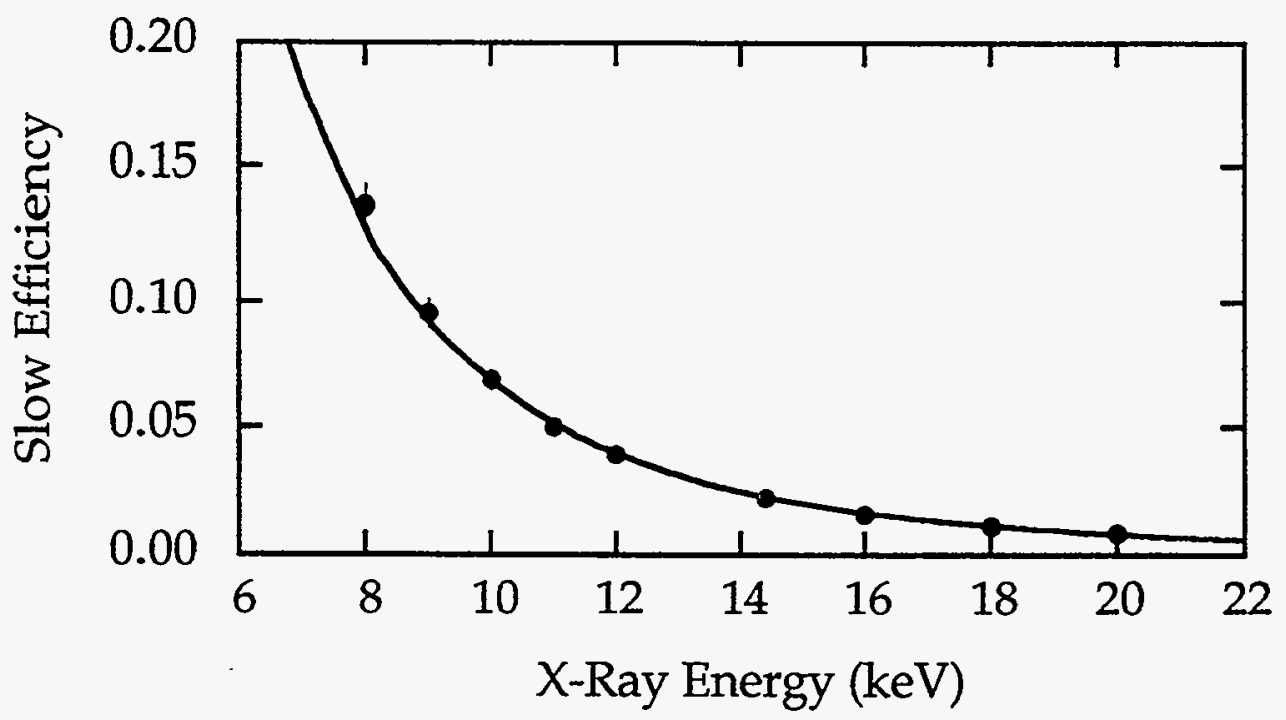

Figure 3.18. Slow efficiency for a beveled edge APD at high gain. The solid line is a fit using equation (3.1). 


\section{Improved Time Response}

It is possible to improve the time response shown in figure 3.16 by increasing the discriminator threshold, though this reduces the efficiency. This is evident in figure 3.19, where the plots from figure 3.16 are reproduced as well as the time response measured with higher thresholds (efficiency reduced by a factor of two). The additional peak that becomes clear in each case is a minibunch of electrons (note the $2.8 \mathrm{~ns}$ periodicity).

The beveled edge device time response improves due to the selective reduction in the events in the tail. This indicates that the events later in the tail have lower peak heights than the events earlier in the tail, which is confirmed by scanning the threshold over a wide range: higher thresholds select events closer to the main peak (see also [Baron and Ruby, 1993, 1]). This suggests that there may be traps in the low field region, that hold electrons for short periods of time. $X$-rays absorbed closer to the front of the low field region (later pulses) generate electrons that pass through more of the low field region and hence have a greater opportunity to be trapped. Additional evidence for trapping in these devices has been presented by Gullikson [Gullikson, 1993, 87] who shows that the rise time after a short pulse ( $10 \mathrm{~ns})$ of $95 \mathrm{eV}$ photons $(0.7 \mu \mathrm{m}$ absorption length) is significantly degraded at lower temperatures (i.e. as the traps become deeper relative to the thermal energy of the carriers). Rise times $\sim \mu$ s were apparent at $-27 \mathrm{C}$.

The improved response for the reach through device at higher thresholds is primarily the result of the reduced effect of the pre-amplifier noise. This also accounts for the overall shift in the response to later times: the preamp noise was at slightly higher frequencies than the signal, so it tended to make the constant fraction discriminator fire early. In fact, though detailed investigations remain to be done, a recent measurement showed that with the MAR- 6 preamplifiers the time resolution, in conditions comparable to that of the measurement in figure 3.16, improves to about $0.6 \mathrm{~ns}$ (FWHM) a little better than the $0.8 \mathrm{~ns}$ in figure 3.16. 


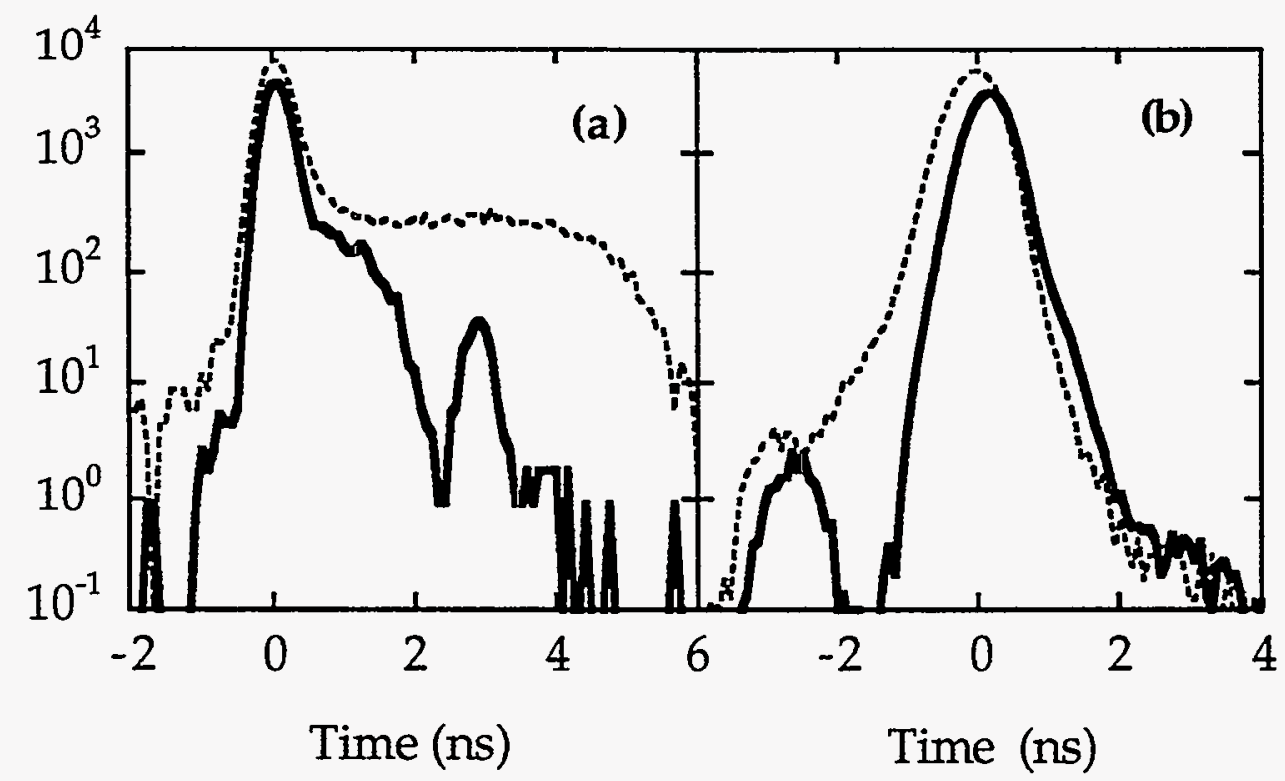

Figure 3.19. Improved time response at higher discriminator threshold for 14 . $\mathrm{keV}$ photons. (a) is the response of a beveled edge diode with the dashed line corresponding to a $10 \%$ efficiency while the solid line is for a higher threshold with a $5 \%$ efficiency. (b) is the response of the EG\&G diode with thresholds corresponding to $20 \%$ and $10 \%$, respectively.

\section{Pulse Height (Charge) Response}

Though the primary interest in this work has been the time response and the efficiency of the various detectors, the pulse height (charge) response of one diode was investigated. The diode was attached to a standard charge sensitive pre-amplifier (EG\&G Ortec 142AH [EG\&G Ortec, 85]) and a shaping amplifier with a 100 ns time constant (EG\&G Ortec 450). The measured pulse charge distributions at 5.9 and $13.4 \mathrm{keV}$ is shown in figure 3.20 for both a point like source $\left(\sim 25 \times 25 \mu \mathrm{m}^{2}\right)$ and a radioactive source illuminating the entire front surface.

The poorer resolution for the full surface illumination is due to the variation in the gain over the surface. The long tail at low energies is due to absorption events occurring within the gain region, with the sharp rise at very low energies due to noise events. The improved (fractional) resolution at higher 
energies indicates that some of the observed width is probably due to amplifier noise. Though this is not the best resolution available with these devices, this does give a feeling for what is possible, without a lot work. For a description of the various factors involved in $\mathrm{x}$-ray spectroscopic measurements see [Webb and McIntyre, 1976, 57]. Finally, note that although one paper [Carrier and Lecomte, $1990,53]$ has suggested that the $x$-ray response of reach through diodes might saturate at high photon energies, no such effects were noted here with the beveled edge device (the peak position scaled linearly from 6 to $16 \mathrm{keV}$ ).

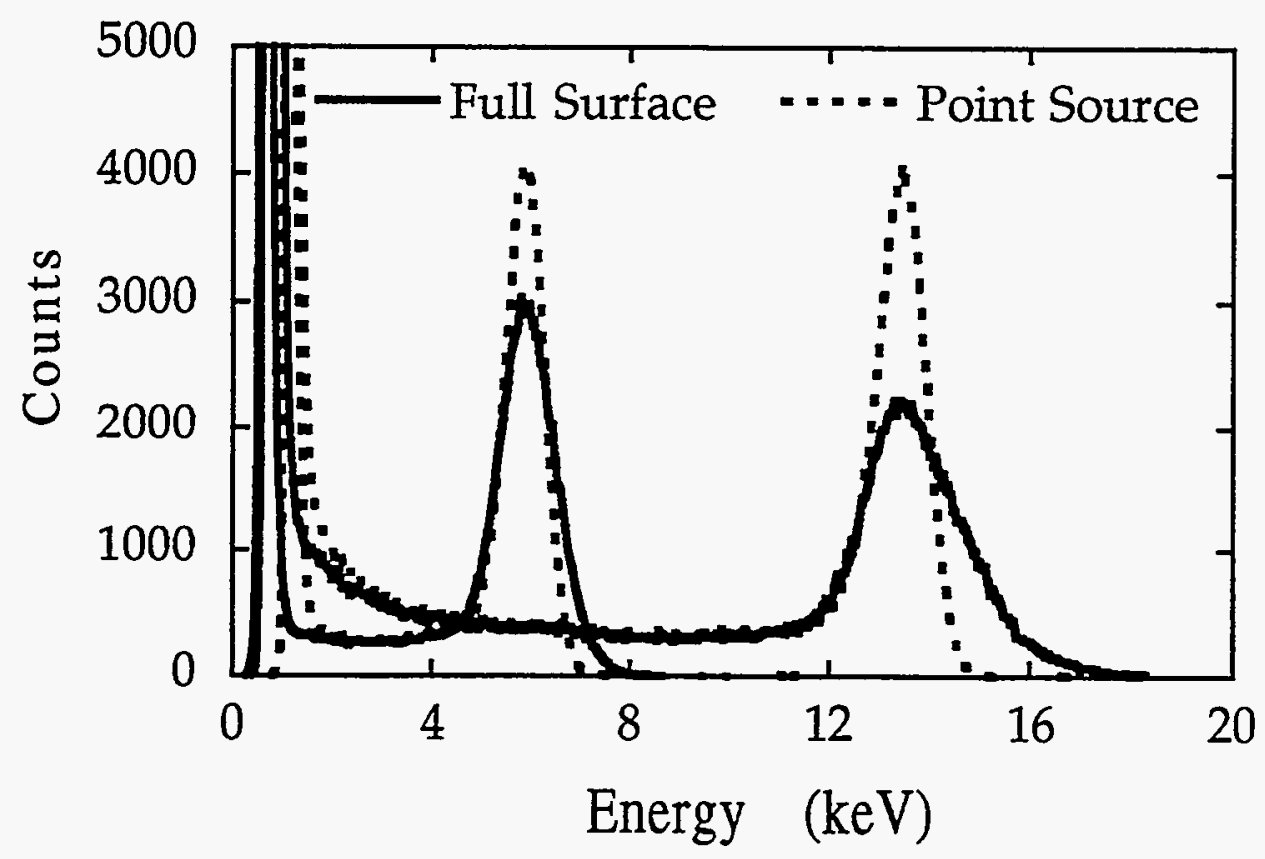

Figure 3.20. Pulse charge distribution from a $1.6 \mathrm{~cm}$ diameter beveled edge diode at a gain of $M \sim 250$. Point and full surface illumination at 5.9 and $13.4 \mathrm{keV}$. The resolutions (FWHM) are 16 and $21 \%$ at $5.9 \mathrm{keV}$ and 8 and $17 \%$ at $13.4 \mathrm{keV}$ for point like and full surface illumination, respectively.

\section{Detector Dependability}

One of the major difficulties with the API beveled edge diodes is that they do not seem to have a long lifetime. Table 3.1 shows the lifetimes and various parameters for 12 diodes ( $1.6 \mathrm{~cm}$ diameter) used by this author. In general, diodes were run no higher than 50 volts below breakdown with a 10 meg resistor 
in series. Of the 12 diodes, two were preliminary designs, three are still in use (having lasted 200 to 300 hours), two were returned to API because the mountings were not correct (but performed very nicely prior to their return) and five have died in various ways, one of these (\#3) being the best diode that we have used.

\begin{tabular}{|c|c|c|c|}
\hline $\begin{array}{l}\text { Diode } \\
\text { \# }\end{array}$ & $\begin{array}{l}\text { Date } \\
\text { Rec. }\end{array}$ & $\mathrm{V}_{\max } / \mathrm{M} / \mu \mathrm{A}$ & Lifetime and Comments \\
\hline 1 & $5 / 92$ & $2430 / 1550 / 8.7$ & Preliminary design. \\
\hline 2 & $11 / 92$ & $\sim 2200 / \sim 400 /-$ & Preliminary design. \\
\hline 3 & $11 / 92$ & $2510 / 2570 / 1.7$ & $\sim 1000$ hrs (died 4/94), Best diode. \\
\hline 4 & $11 / 92$ & $2200 / 650 / 1.0$ & >200 hrs, Noisy. \\
\hline 5 & $4 / 93$ & $2210 / 740 / 3.5$ & $\begin{array}{l}>200 \text { hrs. (returned to API for one } \\
\text { with correct mounting) }\end{array}$ \\
\hline 6 & $4 / 93$ & $2230 / 500 / 3.6$ & As 5 , above. \\
\hline 7 & $6 / 93$ & $2510 / 4500 / 6.5$ & $\sim 200 \mathrm{hrs}$ (large dark current increase) \\
\hline 8 & $6 / 93$ & $2230 / 560 / 1.4$ & 200 hrs (large dark current increase) \\
\hline 9 & $4 / 94$ & $2470 / 1040 / 3.0$ & $\sim 200 \mathrm{hrs}($ died $4 / 94)$ \\
\hline 10 & $4 / 94$ & $2250 / 900 / \sim 2$ & $>300 \mathrm{hrs}$ (still functioning) \\
\hline 11 & $5 / 94$ & $2490 / 1200 / \sim 7$ & $>200$ hrs (still functioning) \\
\hline 12 & $5 / 94$ & $2490 / 1200 / 3.5$ & $>200 \mathrm{hrs}$ (still functioning) \\
\hline
\end{tabular}

Table 3.1 History of $1.6 \mathrm{~cm}$ API beveled edge diodes. Shows date received, the parameters just below breakdown (voltage, gain and current - as measured by API) and approximate lifetime. Note that diodes 3,5 and 6 correspond to diodes 1,2 and 3 of [Baron and Ruby, $1993,1]$.

Table 2.1 shows that the beveled edge diodes have not been incredibly reliable. However, the fact that the three most recently received diodes are still in operation seems a hopeful sign. Discussions with Shawn Fagen of API indicated that, as of $8 / 94$, API knew about the problem, but had not yet worked out the causes.

The EG\&G devices have only been tested for shorter periods of time ( $>300$ hours for one device and $\sim 200$ hours for another) but of the two investigated, no 
degradation in their response with time has been noted. This author has heard that there can be problems with these diodes absorbing moisture and failing, however, this has only been with unsealed devices. The devices used here are sealed (at the back where the junction is located) and there has been no indication of any problems.

\section{Recent Results: High Efficiencies and High Rates}

This author has used APD detectors in some recent nuclear scattering experiments. Though a detailed investigation of the detector response was not carried out, a few measurements were made. In particular, we looked at the response of the EG\&G diodes with the MAR-6 amplifiers and the response of the EG\&G and API diodes at very high rates.

The efficiency of the EG\&G diodes at $14.4 \mathrm{keV}$ could be made quite high by using a grazing incidence geometry. For a detector acceptance of $2.3 \mathrm{~mm} \times 10$ $\mathrm{mm}$ (grazing angle 13 degrees) the efficiency was $57 \pm 3 \%$ relative to an $\mathrm{NaI}(\mathrm{Tl}$ ) detector (which is nearly $100 \%$ efficient) while for an acceptance of $1.1 \mathrm{~mm} \times 10$ $\mathrm{mm}$ ( 6 degrees) the efficiency was $74 \pm 4 \%$. In addition, the time resolution of the EG\&G devices was seen to improve with the MAR- 6 amplifiers, decreasing from about $0.8 \mathrm{~ns}$ with the 6954 to about to about $0.6 \mathrm{~ns}$. (FWHM). Finally, the EG\&G devices were routinely used at prompt count rates of about $3 \mathrm{MHz}$ at $14.4 \mathrm{keV}$ and were used at rate of about $15 \mathrm{MHz}$ at $6.2 \mathrm{keV}$.

One API diode was also used at very high prompt rates, $77 \mathrm{MHz}$, at 9.4 $\mathrm{keV}$. The output from the diode in this case is shown in figure 3.21. The prompt rate of $77 \mathrm{MHz}$ means that about $14 \mathrm{x}$-rays were detected in each prompt flash. Note that about $40 \mathrm{~ns}$ was required for the tail of the prompt pulse to die away. Also, it was crucial to add a small negative DC offset to the amplified signal from the diode before feeding it into the discriminator. This was the result of using a capacitively coupled system: the average current through such a system must be zero so the large negative going prompt pulses introduce a positive offset in the baseline. Without correcting the offset, the discriminator would not have fired on single photon events. Finally, it is worth noting that any measurement of a time response under such high rate conditions should be made with care: one 
must check that the detector response is uniform over the delayed counting window. This can be done by tuning the incident energy far from the nuclear resonance energy (but preserving the same prompt count rate) and placing a radioactive source near the detector. This should lead to a count rate that is uniform in time.

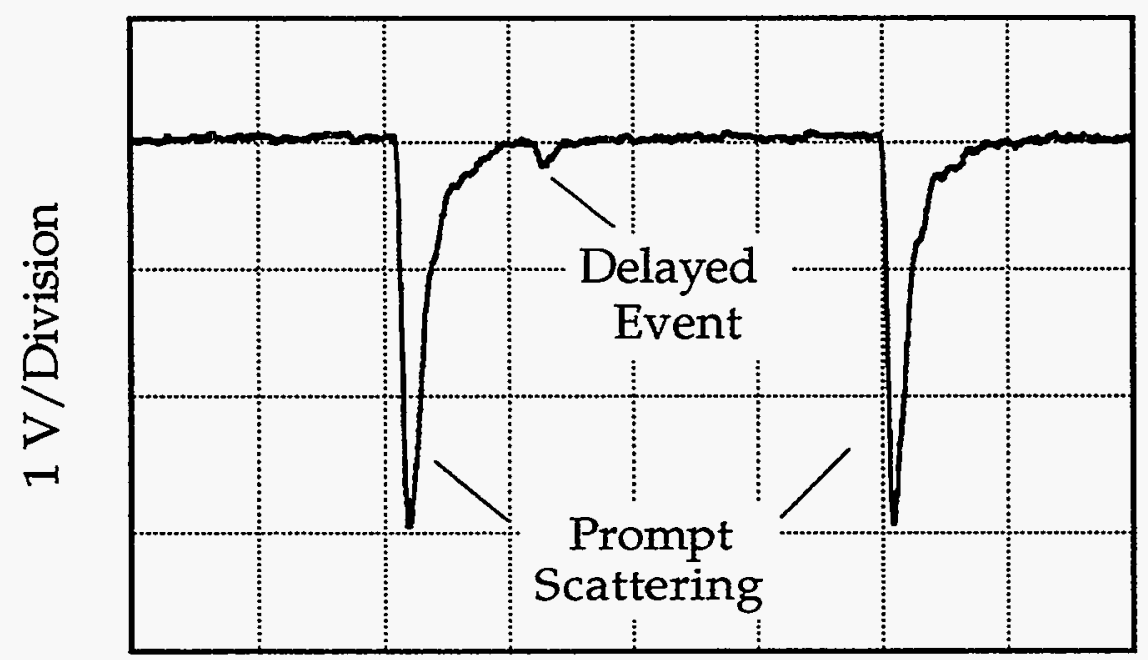

50 ns/Division

Figure 3.21. Scope trace of the output of an API diode at a prompt rate of about $14 \mathrm{x}$-rays/bunch. A single delayed event is shown between two prompt flashes. Diode output has been amplified by $10^{3}$.

\section{References for Chapter 4}

1 A.Q.R. Baron and S.L. Ruby, Time Resolved Detection X-rays Using Large Area Avalanche Photodiodes. Nuclear Instruments And Methods In Physics Research A 343 (1993) 517.

2 A.Q.R. Baron, Report on the X-ray Efficiency and Time Response of a $1 \mathrm{~cm}^{2}$ Reach Through Avalanche Diode. Nuclear Instruments and Methods In Physics Research A 353 (1994) 665. 
3 G. Faigel, D.P. Siddons, J.B. Hastings, P.E. Haustein, J.R. Grover, J.P. Remeika, and A.S. Cooper, New Approach to the Study of Nuclear Bragg Scattering of Synchrotron Radiation. Phys. Rev. Lett. 58 (1987) 2699.

4 Bicron, 12345 Kinsman Road, Newbury Ohio 44065-9577, USA, Tel: (216) 564 2251, Fax: (216) 5648047.

5 J. Arthur, private communication (1994).

6 Hamamatsu, 360 Foothill Road, PO Box 6910, Bridgewater, New Jersey, 088070910, USA, Tel: 201231 0960, Fax: 2012311539.

7 J. Metge, R. Rüffer, and E. Gerdau, A gamma-ray detector with good time resolution and high signal-to-noise ratio. Nuclear Instruments and Methods in Physics Research A292 (1990) 187.

8 Philips Components, 2001 West Blue Heron Blvd., PO Box 10330, Riviera Beach, Fl, USA, Tel: (407) 881 3200, .

9 U. Bergmann, S.D. Shastri, D.P. Siddons, B.W. Batterman, and J.B. Hastings, Temperature dependence of nuclear forward scattering of synchrotron radiation in alpha- ${ }^{57} \mathrm{Fe}$. Phys. Rev. B 50 (1994) 5957.

10 S. Shastri, private communication (1994).

11 S.L. Ruby and G. Tirsell, Delayed pulse at 15 ns in Bicron 420. (1992).

12 J.L. Wiza, Microchannel plate detectors. Nucl. Instrumen. and Meth. 162 (1979) 587.

13 E.H. Eberhardt, Gain model for microchannel plates. Applied Optics 18 (1979) 1418.

14 E. Gerdau, private communication (1993).

15 J.D. Kilkenny, High speed proximity focused $x$-ray cameras. Laser and Particle Beams 9 (1991) 49.

16 O.H.W. Siegmund, J. Vallerga, and B. Wargelin, Background events in microchannel plates. IEEE Trans. Nucl. Sci. 35 (1988) 524.

17 Galileo Electro-Optics, Galileo Park, Sturbridge, Ma 01566, Tel: (508) 347 9191, Fax: (508) 3849. 
18 Buckbee-Mears, 245 East 6th Street, St., Paul, MN 55101, Tel: (612) 228 6400, Fax: (612) 2286572.

19 UltraVac, 200 Littlefield Avenue, South SanFrancisco, CA 94080 USA, Tel: (415) 873 8783, Fax: (415) 5949177.

20 Varian Vacuum Products, 121 Hartwell Avenue, Lexinton MA, 02173 USA, Tel: (617) 8617200 , Fax: (617) 8605437.

21 MDC, 23842 Cabot Boulevard, Hayward, CA 94545-1651, USA, Tel: (510) 887-6100, Fax: (510) 887-0626.

22 Directed Energy Incorporated (DEI), 2301 Research Blvd. Ste. 101, Fort Collins, Colorado, 80526, USA, Tel: (303) 493 1901, Fax: (303) 4931903.

23 G. Krausse (DEI), private communication (1991).

24 G.W. Fraser, J.F. Pearson, and J.E. Lees, Dark noise in microchannel plate $x$-ray detectors. Nuclear Instruments and Methods in Physics Research A254 (1986) 447.

$25 \mathrm{KDI} /$ Triangle Electronics, 60 South Jefferson Rd., Whippany, NJ 07981-1001 USA, Tel: (201) 884-3952, Fax: (201) 884394.

26 J.E. Bateman, The detection of hard $x$-rays $(10-140 \mathrm{keV})$ by channel plate electron multipliers. Nucl. Instrumen. and Meth. 144 (1977) 537.

27 G.W. Fraser, The soft $x$-ray quantum detection efficiency of microchannel plates. Nucl. Instrumen. and Meth. 195 (1982) 523.

28 E.O. Kane, Simple Model for Collision Effects in Photoemission. Phys. Rev. 147 (1966) 335.

29 B.L. Henke, J. Lisegang, and S.D. Smith, Soft-x-ray induced secondary-electron emission from semiconductors and insulators: Models and measurements. Phys. Rev. B 19 (1979) 3004.

30 L.G. Eliseenko, V.N. Shchemelev, and M.A. Rumsh, Quantum yields of the surface $x$-ray photoeffect at 1-10 Angstoms. Zhurnal Tekhnicheskoi Fiziki [Sov. Phys. Tech. Phys. 13 (1968) 122] 38 (1968) 175.

31 P.J. Ebert, J.L. Gaines, and G.R. Leipelt, Measurement of $x$-ray-induced electron currents from metal targets. Lawrence Radiation Laboratory (presently Lawrence Berkeley Laboratory), Rep. No. UCRL-50691 (1969). 
32 J.L. Gaines and R.A. Hansen, X-ray induced electron emission from thin gold foils. J. Appl. Phys. 47 (1976) 3923.

33 R.H. Day, P. Lee, E.B. Saloman, and D.J. Nagel, Photoelectric quantum efficiencies and filter window absorption coefficients from $10 \mathrm{eV}$ to $10 \mathrm{keV}$. J. Appl. Phys. 52 (1981) 5965.

34 B.L. Henke, J.P. Knauer, and K. Premaratne, The characterization of $x$-ray photocathodes in the 0.1-10-keV photon energy region. J. Appl. Phys. 52 (1981) 1509.

35 R.L. Bell, Negative electron affinity devices. Oxford: Clarendon Press (1973).

36 A.L. Andrushchenko, V.N. Shchemelev, G.B. Stuchinkskii, and A.I. Klimin, Quantum yield of the $x$-ray photelectric effect in negative electron affinity emitters. Zh. Eksp. Teor. Fiz. [JETP 67, 1106-1110 (1989)] 94 (1988) 38.

37 E.M. Gullikson and B.L. Henke, X-ray-induced secondary electron emission from solid xenon. Phys. Rev. B 39 (1989) 1.

38 J.M. Munier, F. Gex, G. Lelievre, D. Bauduin, P. Bonnay, J. Gauthey, J.P. Gex, and C. Cavailler, Performances of $a \mathrm{Xe-solid} x$-ray photocathode. Proceedings of the SPIE 1140 (1989) 566.

39 M.J. Whiteley, J.F. Pearson, G.W. Fraser, and M.A. Barstow, The Stability of CsI-Coated Microchannel Plate X-ray Detectors. Nuclear Instruments and Methods in Physics Research 224 (1984) 287.

40 K. Premaratne, E.R. Dietz, and B.L. Henke, The stability of cesium iodide $x$-ray photocathodes. Nucl. Instrumen. and Meth. 207 (1983) 465.

41 A. Lightstone, A. MacGregor, D. MacSween, R. McIntyre, C. Trottier, and P. Webb, Photon counting modules using RCA silicon avalanche photodiodes. Electronic Engineering 61 (1989) 37.

42 P.P. Webb, R.J. McIntyre, and J. Conradi, Properties of Avalanche Photodiodes. RCA Review 35 (1974) 234.

43 S.M. Sze, Physics of Semiconductor Devices. 2nd ed. New York: John Wiley and Sons Inc. (1981).

44 A.R. Jones, $A$ comparison of two types of amplifying radiation detector for use in portable gamma radiation monitors. IEEE Trans. Nucl. Sci. NS-20 (1973) 526.

45 P.P. Webb and A.R. Jones, Large Area Reach Through Diodes for Radiation Monitoring. IEEE Trans. Nucl. Sci. NS-21 (1974) 151. 
46 S. Kishimoto, An Avalanche Photodiode Detector for X-Ray Timing Measurements. Nuclear Instruments and Methods in Physics Research A 309 (1991) 603.

$47 \mathrm{~S}$. Kishimoto, High time resolution $x$-ray measurements with an avalanche photodiode detector. Rev. Sci. Instrumen. 63 (1992) 824.

48 S.L. Ruby, private communication (1992).

49 Advanced Photonix Inc., 1240 Avenida Acaso, Camarillo, Ca, 93012, Tel: (805) 987 0146, Fax: (805) 4849935.

50 EG\&G Optoelectronics Canada, 22001 Dumberry Road, Vaudreuil, Quebec J7V 8P7, Tel: (514) 424 3300, Fax: (514) 4243411.

51 Radiation Monitoring Devices, 44 Hunt Street, Watertown Ma 02171, Tel: (617) 926 1167, Fax: (617) 9269743.

52 R. Lecomte, C. Martel, and C. Carrier, Status of BGO-Avalanche Potodiode Detectors for Spectroscopic and Timing Measurments. Nuclear Instruments and Methods in Physics Research A278 (1989) 585.

53 C. Carrier and R. Lecomte, Recent Results In Scintillation Detection With Silicon Avalanche Photodiodes. IEEE Trans. Nucl. Sci. 37 (1990) 209.

54 C. Carrier and R. Locomte, Timing performance of scintillators read out by silicon avalanche photodiodes. Nuclear Instruments and Methods in Physics Research A299 (1990) 115.

55 K.M. James, M.J. Masterson, and R. Farrell, Performance evaluation of new largearea avalanche photodiodes for scintillation spectroscopy. Nuclear Instruments and Methods in Physics Research A313 (1992) 196.

56 R. Raylman, private communication (1993).

57 P.P. Webb and R.J. McIntyre, Large Area Reach-Through Avalanche Diodes for Xray Spectroscopy. IEEE Trans. Nucl. Sci. NS-23 (1976) 138.

58 R. Farrell, K. Vanderpuye, G. Entine, and M.R. Squillante, High Resolution, Low Energy Avalanche Photodiode X-Ray Detectors. IEEE Trans. Nucl. Sci. 38 (1991) 144.

59 E.M. Gullikson, E. Gramsch, and M. Szawlowski, The Soft X-Ray Detection Efficiency Of Large-Area Avalanche Photodiodes. To be published. (1994). 
60 J.R. Palmer and G.R. Morrison, The use of avalanche photodiodes for the detection of soft $x$ rays. Rev. Sci. Instrumen. 63 (1992) 828.

61 S. Cova, A. Lacaita, M. Ghioni, and G. Ripamonti, 20-ps timing resolution with silicon avalanche photodiodes. Rev. Sci. Instrumen. 60 (1989) 1104.

62 J.A. Hauger, Y. Choi, A.S. Hirsch, R.P. Scharenberg, B.C. Stringfellow, M.L. Tincknell, N.T. Porile, G. Rai, J. Garbarino, and R.J. McIntyre, A time-offlight detector based on silicon avalanche diodes. Nuclear Instruments and Methods in Physics Research A377 (1994) 362.

63 P. Cushman and R. Rusack, A Photomultiplier Tube Incorporating An Avalanche Photodiode. Nuclear Instruments and Methods in Physics Research A333 (1993) 381.

64 M. Szawlowski, E. Gramsch, S. Zhang, and M. Madden, Performance of the Vacuum Avalanche Photodiode. Advanced Photonix, Inc. , Rep. No. (1993).

65 M.R. Squillante, R. Farrell, J.C. Lund, F. Sinclair, G. Entine, and K.R. Keller, Avalanche Diode Low Energy X-ray and Nuclear Particle Detector. IEEE Trans. Nucl. Sci. 33 (1986) 336.

66 P.P. Webb and B. Dion. High speed 128-element avalanche photodiode array for optical computing applications. in SPIE Conference on Optical Enhancements to Computing Technology. 1991. p. 236.

67 E. Gramsch, S. Zhang, M. Madden, M. Lindberg, and M. Szawlowski, High density avalanche photodiode array. Proceedings of the SPIE 2022 (1993).

68 R.J. McIntyre, The Multiplication Noise in Uniform Avalanche Junctions. IEEE Transactions on Electron Devices ED-13 (1966) 164.

69 R.J. McIntyre, The Distribution of Gains In Uniformly Multiplying Avalanche Photodiodes: Theory. IEEE Transactions on Electron Devices ED-19 (1972) 703.

70 API, Noise Properties of Advanced Photonix Avalanche Photodiodes. , Rep. No. .

71 V.L. Gelezunas, W. Siebt, and G. Huth, Uniform large-area high-gain silicon avalanche radiation detectors from transmutation doped silicon. Appl. Phys. Lett. 30 (1977) 118.

72 API, Response Time Characteristics of Advanced Photonix Avalanche Photodiodes. , Rep. No. . 
73 M.J. Szawlowski, S. Zhang, A. DeCecco, M. Madden, M. Lindberg, and E. Gramsch. Performance of Large Area Avalanche Photodiodes. in Conference record of the 1992 IEEE Nuclear Science Symposium and Medical Imaging Conference (Cat. No. 92CH3232-6). 1, 1992. Orlando, Fl p. 239.

74 R. Farrell, K. Vanderpuye, L. Cirignano, M.R. Squillante, and G. Entine, Radiation detection performance of very high gain avalanche photodiodes. To be published. (1994).

75 W.H. McMaster, N.K.D. Grande, J.H. Mallet, and J.H. Hubbell, Compilation of $X$-ray Cross Sections. Lawrence Radiation Laboratory, Rep. No. UCRL50174 Sec. III Rev1 (1969).

76 R.D. Ryan, Precision Measurements of the Ionization Energy and Its Temperature Variation in High Purity Silicon Radiation Detectors. IEEE Trans. Nucl. Sci. NS 20 (1973) 473.

77 J.C. Ashley, C.J. Tung, R.H. Ritchie, and V.E. Anderson, Calculations of Mean Free Paths and Stopping Powers of Low Energy Electrons $(<=10 \mathrm{keV})$ in Solids Using a Statistical Model. IEEE Trans. Nucl. Sci. NS 23 (1976) 1833.

78 L. Katz and A.S. Penfold, Range-Energy Relations for Electrons and the Determination of Beta-Ray End-Point Energies by Absorption. Rev. Mod. Phys. 24 (1952) 28.

79 C. Jacoboni, C. Canali, G. Ottaviani, and A.A. Quaranta, A Review Of Some Charge Transport Properties Of Silicon. Solid-State Electronics 20 (1977) 77.

80 G.L. Miller, D.V. Lang, and L.C. Kimerling, Capcitance Transient Spectroscopy. Annual Review of Material Science 7 (1977) 377.

81 H.D. Nuhn, private communication (1992).

82 W.R. Phillips, I. Ahmad, D.W. Banes, B.G. Glagola, W. Henning, W. Kutschera, K.E. Rehm, J.P. Schiffer, and T.F. Wang, Charge-State Dependence of Nuclear Lifetimes. Phys. Rev. Lett. 62 (1989) 1025.

83 P. Horowitz and W. Hill, The Art of Electronics. New York: Cambridge University Press (1980).

84 Mini-Circuits, P.O. Box 350166, Brooklyn, New York, 11235-0003, Tel: (718) 934 4500, Fax: (718) 3324661.

85 EG\&G Ortec, 100 Midland Road, Oak Ridge TN 37831-0895, Tel: (615) 482 4411, Fax: (615) 4830396. 
86 LeCroy, 700 Chesnut Ridge Road, Chesnut Ridge NY 10977-6499, Tel: (914) 578 6064, Fax: (914) 4258967.

87 E. Gullikson, private communication (1993). 


\section{The Optical Theory for Homogeneous Media and Ideal Multilayers}

\section{Introduction}

This chapter presents the optical theory for the interaction of $\mathrm{x}$-rays with thin films. The results in this chapter are not surprising, and in fact, those for isotropic materials have been presented in various forms on many occasions. However, a careful derivation of the optical approach beginning at the level of Maxwell's equations is difficult to find in the literature, where starting points are frequently vague and approximations are typically made early on. In addition, to this author's knowledge, a general optical approach for $\mathrm{x}$-ray response of anisotropic materials has not been presented in the literature, though one method valid in some special cases for grazing angles of incidence has been presented [Hannon, et al., 1985, 1] and a more general form, also valid only at grazing incidence has recently been presented [Irkaev, et al., 1993, 2].

\section{The X-ray Wave Equation in a Dielectric Material}

The optical and diffraction theories of $x$-ray interaction with matter both may begin with a wave equation for the fields inside a material at $x$-ray frequencies. Ideally, one would begin this derviation with the microscopic Maxwell equations (see, e.g. JJackson, 1975, 3] p. 227). These are (in Gaussian units)

$$
\begin{aligned}
& \nabla \cdot \mathbf{b}=0 \\
& \nabla \bullet \mathbf{e}=4 \pi \rho \\
& \nabla \times \mathbf{e}=-\frac{1}{c} \frac{\partial \mathbf{b}}{\partial t}
\end{aligned}
$$




$$
\nabla \times \mathbf{b}=\frac{1}{c} \frac{\partial e}{\partial t}+\frac{4 \pi}{c} \mathbf{j}
$$

where $\mathbf{e}$ and $\mathbf{b}$ are the microscopic fields and $\rho$ and $\mathbf{j}$ the microscopic charge and current densities. However, these equations include all the details of the atomic and molecular structure (e.g. coulombic forces) which are not of interest in calculating the response of a material at $x$-ray frequencies. This is because, to a very good approximation, the structure (charge density) of the solid will not be affected by the presence of the weak $x$-ray field at very high frequencies. Thus, we separate out the $x$-ray fields and the currents induced by those fields, but retain the microscopic charge distribution (see also the discussion in [Landau, et al., 1984, 4] pp. 440-441). The equations become

$$
\begin{aligned}
& \nabla \cdot \mathbf{B}=0 \\
& \nabla \bullet E=4 \pi \rho \\
& \nabla \times E=-\frac{1}{c} \frac{\partial B}{\partial t} \\
& \nabla \times B=\frac{1}{c} \frac{\partial E}{\partial t}+\frac{4 \pi}{c} J
\end{aligned}
$$

where the capitalized quantities indicate that we have focussed on the $x$-ray induced fields and currents in the material. Defining D by (see e.g. [Agranovich and Ginzburg, 1966, 5] [Ginzburg, et al., 1962, 6])

$$
\frac{\partial \mathrm{D}}{\partial \mathrm{t}}=\frac{\partial \mathrm{E}}{\partial \mathrm{t}}+4 \pi \mathrm{J}
$$

the equations (4.2) may be re-written as

$$
\begin{aligned}
& \nabla \cdot \mathbf{B}=0 \\
& \nabla \cdot \mathrm{D}=0 \\
& \nabla \times \mathrm{E}=-\frac{1}{\mathrm{c}} \frac{\partial \mathrm{B}}{\partial \mathrm{t}}
\end{aligned}
$$




$$
\nabla \times B=\frac{1}{c} \frac{\partial D}{\partial t}
$$

Equation (4.4b) is a consequence of charge conservation*. Then noting that (4.4a) and $(4.4 \mathrm{~b})$ are satisfied if $(4.4 \mathrm{c})$ and $(4.4 \mathrm{~d})$ are, (apply $\nabla \bullet$ to $4.4 \mathrm{c}$ and $4.4 \mathrm{~d}$ and then use $\nabla \cdot(\nabla \times \mathbf{A})=0$, where $\mathbf{A}$ is any vector) these equations may be reduced to the desired wave equation

$$
\nabla \times \nabla \times E=-\frac{1}{c^{2}} \frac{\partial^{2} \mathbf{D}}{\partial t^{2}}
$$

Finally, the wave equation may be converted to wave-vector and frequency space $(k, \omega)$ through the usual Fourier transform relationships (A represents any vector quantity) $)^{+}$

$$
\begin{aligned}
& \mathbf{A}(\mathbf{k}, \omega)=\int \mathrm{d} \mathbf{x} \mathrm{dt} \mathrm{e}^{-\mathrm{i}(\mathbf{k} \bullet x-\omega t)} \mathbf{A}(\mathbf{x}, \mathrm{t}) \\
& \mathbf{A}(\mathbf{x}, \mathrm{t})=\frac{1}{(2 \pi)^{4}} \int \mathrm{d} \mathbf{k} \mathrm{d} \omega \mathrm{e}^{+\mathrm{i}(\mathbf{k} \cdot x-\omega t)} \mathbf{A}(\mathbf{k}, \omega)
\end{aligned}
$$

Thus equation (4.5) becomes

$$
\mathbf{D}(\mathbf{k}, \omega)=\frac{c^{2}}{\omega^{2}}[(\mathbf{k} \bullet \mathbf{k}) \mathrm{E}(\mathbf{k}, \omega)-(\mathbf{k} \bullet \mathrm{E}(\mathbf{k}, \omega)) \mathbf{k}]
$$

Where the vector identity $\mathbf{k} \times(\mathrm{k} \times \mathrm{E})=(\mathrm{k} \bullet \mathrm{E}) \mathrm{k}-(\mathrm{k} \bullet \mathrm{k}) \mathrm{E}$ has been used.

\section{Constitutive Relations}

One requires a constitutive relationship between $\mathbf{E}$ and $\mathbf{D}$ in order to proceed. X-rays interact weakly with matter so it is sufficient to assume a linear

\footnotetext{
* It is not necessarily clear that $\nabla \bullet \mathrm{J}+\mathrm{d} \rho / \mathrm{dt}=0$ is valid in this case, as $\rho$ is the microscopic charge while $J$ is the $x$-ray induced current. However, we assume that any high frequency component to the charge density is just that induced by the $x$-ray field.

+ Explicitly, a plane wave has $\mathbf{A}(\mathbf{k})=\delta\left(\mathbf{k}-\mathbf{k}^{\prime}\right)$ so its spatial dependence is $\mathbf{A}_{\mathbf{k}^{\prime}}(\mathbf{x}) \propto \mathrm{e}^{+\mathbf{i} \mathbf{k}^{\prime} \bullet \mathbf{x}}$
} 
relationship. Then, most generally, one has (see, e.g., [Agranovich and Ginzburg, 1966, 5])

$$
D_{i}(x, t)=\int d t^{\prime} \int d x^{\prime} \varepsilon_{i j}\left(t-t^{\prime}, x, x^{\prime}\right) E_{j}\left(x^{\prime}, t^{\prime}\right)
$$

where repeated indices are to be summed over. The use of the argument $t-t^{\prime}$ assumes that the problem is time invariant. $\varepsilon$ is also assumed causal, $\varepsilon_{i j}\left(t-t^{\prime}<0, \mathbf{x}, \mathbf{x}^{\prime}\right)=0$.

Equation (4.8) may be transformed to $(k, \omega)$ space using

$$
\begin{aligned}
& \mathbf{D}(\mathbf{k}, \omega)=\int d x d t e^{-i k \bullet x} e^{+i \omega t} D(x, t) \\
& \varepsilon_{i j}\left(t-t^{\prime}, \mathbf{x}, x^{\prime}\right)=\frac{1}{(2 \pi)^{7}} \int d k_{1} d k_{2} d \omega_{1} \\
& x e^{+i k_{1} \bullet x} e^{+i k_{2} \bullet x^{\prime}} e^{-i \omega_{1}\left(t-t^{\prime}\right)} \varepsilon_{i j}\left(k_{1}, k_{2}, \omega_{1}\right) \\
& E\left(x^{\prime}, t^{\prime}\right)=\frac{1}{(2 \pi)^{4}} \int d k_{3} d \omega_{2} e^{+i k_{3} \bullet x^{\prime}} e^{-i \omega_{2} t^{\prime}} E\left(k_{3}, \omega_{2}\right)
\end{aligned}
$$

Equation (4.8) then reduces to

$$
\mathbf{D}_{\mathrm{i}}(\mathbf{k}, \omega)=\frac{1}{(2 \pi)^{3}} \int \mathrm{d} \mathbf{k}^{\prime} \varepsilon_{\mathrm{ij}}\left(\mathbf{k},-\mathbf{k}^{\prime}, \omega\right) \mathbf{E}_{\mathbf{j}}\left(\mathbf{k}^{\prime}, \omega\right) \quad\left(\begin{array}{l}
\text { Linear } \\
\text { Material }
\end{array}\right)
$$

The Dielectric Tensor in Crystalline and Homogeneous Materials

At this point, one must make additional assumptions about the material in order to continue easily. For the bulk of this chapter, only the result for a homogeneous material is required. However, since a homogeneous material may be taken as a limiting case of a crystal (the limit in which it is translationally 
invariant for any displacement), I take the small additional space here to derive the crystal result. In a crystal, the material response is invariant under translation by any lattice vector, $\mathbf{R}$. One has

$$
\varepsilon_{\mathrm{ij}}\left(t, x, x^{\prime}\right)=\varepsilon_{\mathrm{ij}}\left(t, x+R, x^{\prime}+R\right)
$$

For the transformed (k-space) quantity, this reduces to the condition that

$$
\varepsilon_{i j}\left(\mathbf{k},-\mathbf{k}^{\prime}, \omega\right)=\mathrm{e}^{\mathrm{i}\left(\mathbf{k}-\mathbf{k}^{\prime}\right) \bullet \mathbf{R}} \varepsilon_{\mathrm{ij}}\left(\mathbf{k},-\mathbf{k}^{\prime}, \omega\right)
$$

Therefore either $\varepsilon_{\mathrm{ij}}\left(k,-\mathrm{k}^{\prime}, \omega\right)=0$ or the exponential factor is one. The condition that the exponential be one is precisely that $k-k^{\prime}=H$, where $H$ is a reciprocal lattice vector for the crystal. Then we may write

$$
\varepsilon_{\mathrm{ij}}\left(\mathbf{k},-\mathbf{k}^{\prime}, \omega\right)=(2 \pi)^{3} \sum_{\mathbf{H}} \delta\left(\mathbf{k}-\mathbf{k}^{\prime}-\mathbf{H}\right) \varepsilon_{\mathrm{ij}}^{\mathrm{H}}(\mathbf{k}, \omega)
$$

Inserting this into (4.10) we have

$$
\mathrm{D}_{\mathrm{i}}(\mathbf{k}, \omega)=\sum_{\mathbf{H}} \varepsilon_{\mathrm{ij}}^{\mathrm{H}}(\mathbf{k}, \omega) \mathrm{E}_{\mathbf{j}}(\mathbf{k}-\mathbf{H}, \omega) \quad(\text { Crystal })
$$

For a homogeneous (but not necessarily isotropic) material the translation $R$ in (4.11) and (4.12) may be any vector. The sum then drops out of (4.14) leaving the simple constitutive relation

$$
\mathbf{D}_{\mathrm{i}}(\mathbf{k}, \omega)=\varepsilon_{\mathrm{ij}}^{0}(\mathbf{k}, \omega) \mathrm{E}_{\mathrm{j}}(\mathbf{k}, \omega) \quad \text { (Homogeneous Material) }
$$

It is very important to note that, within this homogeneous limit, the dielectric tensor only depends on the direction of propagation. It does not depend on the momentum transfer $\left(\mathbf{k}-\mathbf{k}^{\mathbf{\prime}}\right.$ or $\left.\mathbf{H}\right)$. This is in sharp distinction to the conventional scattering theory used to describe either the scattering of a crystal (equation 4.14), or that of a single atom. In these cases the scatterer is not homogeneous, having internal structure, either that of the electron orbitals or that of the crystal lattice (or both). However, rigorously speaking the assumption of a homogenous sample means there is no internal structure, and hence no dependence on the momentum transfer. Thus the theory presented here is only 
strictly valid for scattering in nearly forward directions, where the internal structure does not matter.

\section{The Dispersion Relation}

Use of the constitutive relation for $\mathbf{D}$ (either 4.14 or 4.15) in the Maxwell equation relating $\mathbf{D}$ and $\mathbf{E}$, equation (4.4b), gives a condition for relating the components of the dielectric tensor and the components of $E(k, \omega)$ and $\mathbf{k}$. Considering only homogeneous materials (and dropping the superscript on $\varepsilon$ ) this is

$$
\overrightarrow{\mathbf{\varepsilon}}(\mathbf{k}, \omega) \mathrm{E}(\mathbf{k}, \omega)=\frac{\mathrm{c}^{2}}{\omega^{2}}[(\mathbf{k} \bullet \mathbf{k}) \mathrm{E}(\mathbf{k}, \omega)-(\mathbf{k} \bullet \mathrm{E}(\mathbf{k}, \omega)) \mathbf{k}]
$$

The arrow on top of the boldface indicates a tensor quantity. Then taking $E^{\lambda}\left(k^{\lambda}, \omega\right)$ to be an eigenvector of the dielectric tensor with eigenvalue $\varepsilon^{\lambda}\left(k^{\lambda}, \omega\right)$ this reduces to

$$
\mathbf{k}^{\lambda} \cdot \mathbf{k}^{\lambda}=\frac{\omega^{2}}{c^{2}} \varepsilon^{\lambda}\left(\mathbf{k}^{\lambda}, \omega\right)
$$

Note that either direct consideration of equation (4.16) or equation (4.4b), requiring $\mathbf{k} \bullet \mathrm{D}(\mathbf{k})=0$, gives that

$$
\mathbf{k}^{\lambda} \cdot \mathrm{E}^{\lambda}\left(\mathbf{k}^{\lambda}, \omega\right)=0
$$

so that the second term on the right hand side of equation (4.16) has been dropped in equation (4.17).

\section{Connection to Quantum Mechanics: The Lorentz Relation}

At this point it becomes appropriate to investigate the form of the dielectric tensor. For $\mathrm{x}$-rays interacting with matter, the Lorentz relation may be 
used to connect $\vec{\varepsilon}$ with the scattering amplitude*. For homogenous, isotropic media this is (see, e.g., [Lax, 1951, 7],[Goldberger and Watson, 1964, 8], p 559, [Sakurai, 1967, 9], p 62)

$$
\varepsilon(\omega)=1+4 \pi \frac{c^{2}}{\omega^{2}} N F(\omega)
$$

$\mathrm{N}$ is the number density of scatterers and $\mathrm{F}$ is their (forward) scattering amplitude. This relationship is also sometimes stated in terms of the index of refraction of the material, $n$, defined by $n^{2}=\varepsilon$. Finally we note that this equation in fact assumes an isotropic distribution of scattering centers. In the case of highly ordered structure, there may be field correlations that modify this result [Lax, 1951, 7].

More generally, we are interested in anisotropic materials. Here a relationship of the form of (4.19) may be assumed to exist for each eigenpolarization in the medium (see, e.g. [Hannon and Trammell, 1969, 10]). Thus (4.19) becomes

$$
\varepsilon^{\lambda}(\mathbf{k}, \omega)=1+4 \pi \frac{c^{2}}{\omega^{2}} N F^{\lambda}(\mathbf{k}, \omega)
$$

where $\lambda$ is the index on the eigenvalues and eigenvectors determined from the forward scattering amplitude.

It is convenient to break $\mathrm{F}^{\lambda}$ into the sum of a contribution from the electronic scattering and one from nuclear scattering. We write

$$
F^{\lambda}(k, \omega)=-r_{e} f(\omega)+F_{n}^{\lambda}(k, \omega)
$$

\footnotetext{
* Note that an alternative is to calculate the induced currents and find the term linear in the applied field. This allows identification of a conductivity tensor, $\bar{\sigma}$, that may be related to the above defined dielectric tensor by [Ginzburg, et al., 1962, 6]

$\vec{\varepsilon}=\vec{I}+i \frac{4 \pi}{\omega} \vec{\sigma}$

where $\overline{\mathrm{I}}$ is the identity matrix.

* Taking $n=1-\delta$ and $k=\omega / c$ one has $\delta=-2 \pi N F(\omega) / k^{2}$
} 
Where $r_{e}=2.818 \times 10^{-15} \mathrm{~m}$ is the classical radius of the electron. The quantity $f(\omega)$ is an oscillator strength for the electronic scattering by an atom, relative to the scattering by a free electron*. It is approximately the number of electrons in the atom, $\mathrm{Z}$. More carefully, of course, $\mathrm{f}$ includes corrections for the atomic structure and for Compton scattering. It will have both real and imaginary parts and one writes $f(\omega)=Z+f^{\prime}(\omega)+$ if' $(\omega)$ (see, e.g., [James, 1962 (Reprinted 1982), 11], chapter 3 ). The corrections have been both measured and calculated (see [McMaster, et al., 1969, 12], [Cromer and Liberman, 1981, 13] and [Henke, et al., $1992,14])$. The negative sign is introduced in (4.21) because $x$-ray frequencies are typically higher than most electronic resonance frequencies so that the scattering amplitude is negative (the sign has no connection to the sign of the electronic charge).

The values of $F_{n}^{\lambda}$ and the eigenvectors are determined from the nuclear scattering amplitude. The amplitude for coherent scattering of a wave with free space wave vector $k_{i}^{v}$ and polarization $\hat{e}_{i}^{v}$ into a wave with wave vector $k_{f}^{v}$ and polarization $\hat{\mathbf{e}}_{\mathrm{f}}^{\mathrm{v}}$ is given as*

$$
F_{n}\left[\left(\hat{\mathbf{k}}_{i}^{v}, \hat{\mathbf{e}}_{i}\right) \rightarrow\left(\hat{\mathbf{k}}_{f}^{v}, \hat{\mathbf{e}}_{f}^{v}\right) ; \omega\right]=\hat{\mathbf{e}}_{f}^{v^{*}} \overrightarrow{\mathbf{M}}\left(\hat{\mathbf{k}}_{\mathrm{i}}^{\mathrm{v}}, \hat{\mathbf{k}}_{f}^{\mathrm{v}}, \omega\right) \hat{\mathbf{e}}_{\mathrm{i}}^{\mathrm{v}}
$$

where $\overline{\mathbf{M}}$ is related to the appropriate T matrix element and may be written as a multipole expansion in vector spherical harmonics. The form of $\overrightarrow{\mathbf{M}}$ is explored in appendix A. For the moment, we only note that choice of an orthogonal pair of polarizations $\hat{\mathbf{e}}_{\mathrm{a}}^{\mathrm{v}}$ and $\hat{\mathbf{e}}_{\mathrm{b}}^{\mathrm{v}}$ allows construction of a scattering tensor in the $\left(\hat{\mathrm{e}}_{\mathrm{a}}^{\mathrm{v}}, \hat{\mathrm{e}}_{\mathrm{b}}^{\mathrm{v}}\right)$ basis:

$$
\overrightarrow{\mathbf{F}}_{\mathbf{n}}\left(\hat{\mathbf{k}}^{\mathrm{v}}, \omega\right)=\left(\begin{array}{ll}
\mathrm{f}_{\mathrm{aa}}\left(\hat{\mathbf{k}}^{\mathrm{v}}, \omega\right) & \mathrm{f}_{\mathrm{ba}}\left(\hat{\mathbf{k}}^{\mathrm{v}}, \omega\right) \\
\mathrm{f}_{\mathrm{ab}}\left(\hat{\mathbf{k}}^{\mathrm{v}}, \omega\right) & \mathrm{f}_{\mathrm{bb}}\left(\hat{\mathbf{k}}^{\mathrm{v}}, \omega\right)
\end{array}\right)
$$

\footnotetext{
* Note that without the assumption of a homogenous medium (equation 4.14 and following), $f$ would depend on the momentum transfer as well as the energy.

** The superscripts " $v$ " are to indicate that these quantities are free space or vacuum quantities. In particular, this means that the wave vectors $\mathbf{k}_{i}^{v}$ and $\mathbf{k}_{f}^{v}$ may be taken as real, without imaginary components.
} 


$$
\mathrm{f}_{\alpha \beta}\left(\hat{\mathbf{k}}^{\mathrm{v}}, \omega\right) \equiv \mathrm{F}_{\mathrm{n}}\left[\left(\hat{\mathbf{k}}^{\mathrm{v}}, \hat{\mathbf{e}}_{\alpha}^{\mathrm{v}}\right) \rightarrow\left(\hat{\mathbf{k}}^{\mathrm{v}}, \hat{\mathbf{e}}_{\beta}^{\mathrm{v}}\right) ; \omega\right]
$$

The (possibly non-orthogonal) eigenvectors and their eigenvalues may then be determined in this basis, and transformed to real space. Given some vacuum wave vector, $\mathbf{k}^{\mathrm{v}}$, one can determine the eigenvectors, $\xi^{\mathrm{v \lambda}}\left(\mathbf{k}^{\mathrm{v}}\right)$, and eigenvalues, $\varepsilon^{\mathrm{v} \lambda}\left(\mathbf{k}^{\mathrm{v}}\right)$.

There is some slight difficulty, however, when the above treatment to determine eigenpolarizations and eigenvalues is combined with the dispersion relation inside the medium, equation (4.17). In particular, the vectors $\mathbf{k}^{\lambda}$ appearing in equation (4.17) will not be the free space wave vectors used with the scattering amplitude. They will, in general, have an imaginary component due to the imaginary part of $\varepsilon^{\lambda}$ and may not be in the same direction as the vacuum wave vector (see the discussion below for planar interfaces). Therefore the eigenvalues $\varepsilon^{\lambda}$ in (4.17) are not obviously related to those obtained from solving the eigenvalue problem in (4.23). However, clarification of this point may be found in Lax's paper[Lax, 1951, 7] where a careful effective field treatment of multiple scattering shows that the appropriate action is to take the wave vectors in (4.22)(4.24) to be the wave vectors in the medium. This, of course, does not completely solve the problem since it is not clear how addition of an imaginary component to the wave vectors in (4.22)-(4.24) is to be treated. However, practically, the imaginary part of the wave vector will be much smaller than the real part and may be neglected in (4.22)-(4.24).

\section{Dispersion Relation with a Planar Interface}

We will be considering the problem of a plane wave incident from vacuum onto a planar boundary. Therefore it is useful to consider the solution to the dispersion relation in this case. In particular, if the wave incident from vacuum has a single well defined k-vector, then satisfying any boundary condition that requires continuity of a field across the interface will require that all waves have the same components of $k$ in the plane of the boundary. Writing $k_{1 l}$ for this component, the dispersion relation in the material, equation (4.17), becomes an equation for the component of $\mathrm{k}$ normal to the interface, $\mathrm{k}_{\mathrm{p}}$, 


$$
\mathrm{k}_{\mathrm{p}}^{\lambda}= \pm \frac{\omega}{\mathrm{c}}\left[\varepsilon^{\lambda}\left(\mathrm{k}_{\mathrm{p}}^{\lambda}, \mathrm{k}_{11}, \omega\right)-\frac{\mathrm{c}^{2}}{\omega^{2}} \mathrm{k}_{11} \cdot \mathrm{k}_{11}\right]^{1 / 2}
$$

And then taking the incident (vacuum) wave vector to have magnitude $k_{0} \equiv \omega / c$ and to make an angle $\theta$, with the interface, this becomes

$$
k_{p}^{\lambda}= \pm k_{0}\left[\varepsilon^{\lambda}\left(k_{p}^{\lambda}, k_{11}, \omega\right)-\cos ^{2} \theta\right]^{1 / 2}
$$

It is useful to re-write (4.26) in terms of the eigenvalue solutions of (4.22) - (4.24), apropos the discussion at the end of the preceding section. This gives

$$
\begin{aligned}
\mathrm{k}_{\mathrm{p}}^{\lambda} & = \pm \mathrm{k}_{0}\left[\varepsilon^{\mathrm{v} \lambda}\left(\hat{\mathbf{k}}^{\mathrm{v} \lambda}, \omega\right)-\cos ^{2} \theta\right]^{1 / 2} \\
& = \pm \mathrm{k}_{0}\left[\sin ^{2} \theta-2 \delta^{\mathrm{v} \lambda}\left(\hat{\mathbf{k}}^{\mathrm{v} \lambda}, \omega\right)\right]^{1 / 2} \\
\hat{\mathbf{k}}^{\mathrm{v} \lambda} & \equiv \frac{\operatorname{Re}\left\{\mathbf{k}^{\lambda}\right\}}{\left|\operatorname{Re}\left\{\mathbf{k}^{\lambda}\right\}\right|}=\frac{\operatorname{Re}\left\{\mathbf{k}_{\mathrm{p}}^{\lambda}\right\} \hat{\mathbf{z}}+\mathbf{k}_{\mathrm{ll}}}{\left(\operatorname{Re}\left\{\mathbf{k}_{\mathrm{p}}^{\lambda}\right\}^{2}+\mathrm{k}_{\mathrm{ll}}{ }^{2}\right)^{1 / 2}}
\end{aligned}
$$

$\hat{\mathbf{z}}$ is the unit vector perpendicular to the interface. This pair of equations may be solved iteratively. First one takes $\hat{\mathbf{k}}^{\mathrm{v} \lambda}$ to be the wave vector incident from vacuum in (4.27) and finds the appropriate component $k_{\mathrm{p}}^{\lambda}$. Then a new $\hat{\mathbf{k}}^{\mathrm{v} \lambda}$ is calculated according to (4.28) which may be used in (4.27), etc. In practice only one or two iterations are typically necessary.

Finally, we consider how many solutions of (4.27) are pertinent to the problem of a plane wave incident on a planar interface. It is clear that for each eigenvalue $\lambda$, equation (4.27) has two solutions corresponding to the two signs from the square root. However, only one of these propagates in (nearly) the direction of the vacuum wave vector and the other may be dropped. Since the construction of the eigenproblem (see the previous section) allows two eigenvalues for each vacuum wave vector, there are 2 possible solutions corresponding to these two values. Finally, complete treatment of the problem must allow a reflected wave in vacuum addition to an incident wave. Therefore 
a second vacuum wave vector must be included and one has four possible waves excited in each material by a single incident (and reflected) wave.

\section{Boundary Conditions at Planar Interfaces}

We now consider the boundary value problem of plane waves at planar interfaces. Of course, this problem has been considered in many different incarnations (see, e.g. [Born and Wolf, 1980,15] for optical problems or [James, 1962 (Reprinted 1982), 11] for x-rays). However, we will use these equations in a slightly novel format, so I derive them below.

I consider the equations appropriate for describing the fields at planar vacuum-dielectric interface, or in a layered structure, where the planar interfaces between all layers are parallel. The in-plane dimensions of the solid are much larger than the wavelength of the radiation, and may be taken as infinite. I also assume that all waves in the media have been excited by an incident plane wave with a single well defined momentum. These assumptions have the important immediate consequence that, in order to meet the boundary conditions at the interfaces, all waves in all materials must have the same in-plane component of k. In addition, assumption of a homogeneous material allows restriction of all wave vectors in the problem to the scattering plane (where the scattering plane is defined as the plane normal to the interface that encompasses the incident wave vector). 


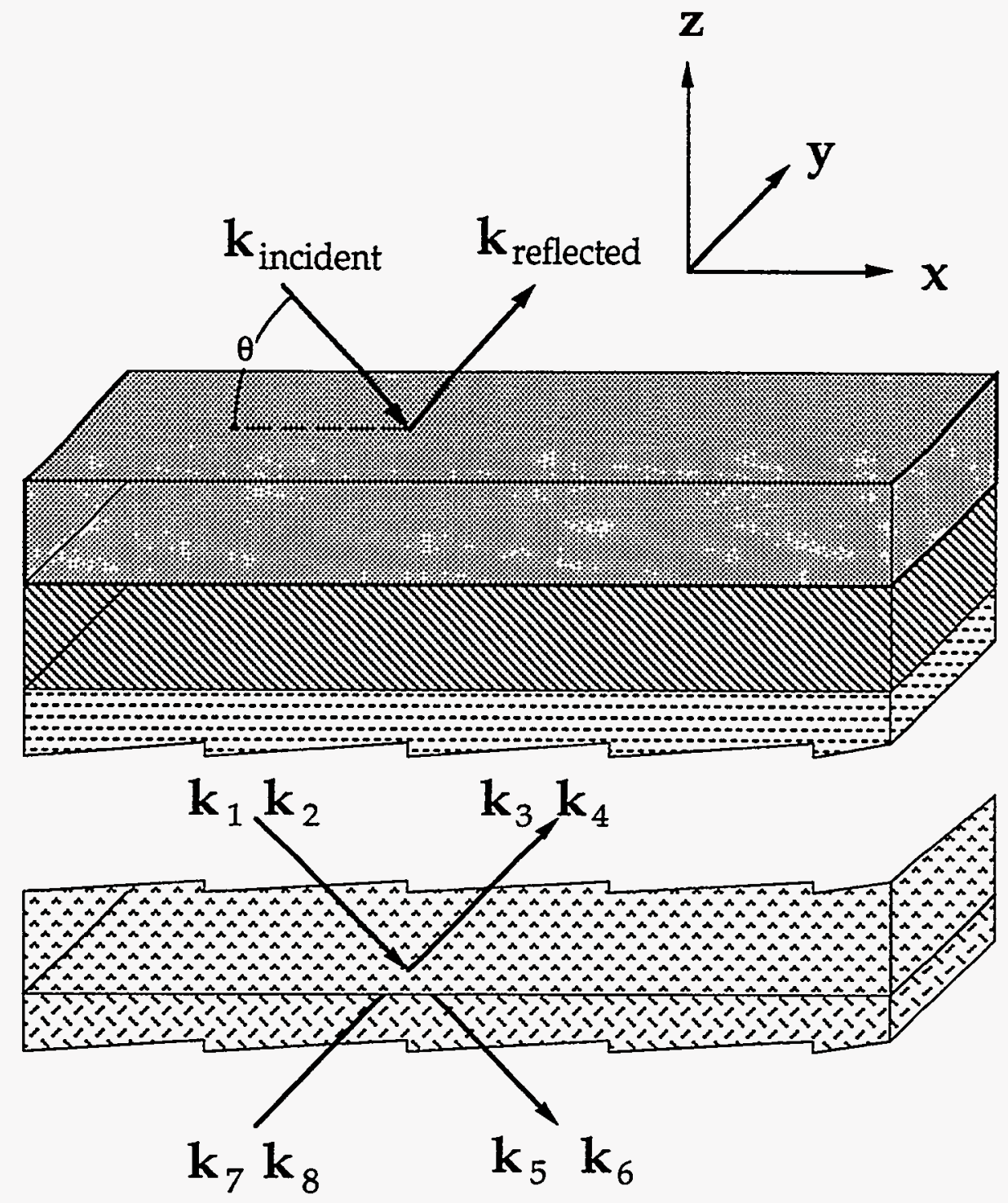

Figure 4.1. Geometry for solution of the boundary value problem of a plane wave incident on a dielectric or dielectric stack.

The problem is then reduced to the situation diagrammed in figure 4.1. All wave vectors are in the $x z$ plane, having independent components $k_{l l}$ and $k_{p}$. $k_{11}$ is taken to be the component of the wave vector in the plane of the interface and $k_{p}$ is the component normal to the interface. Note that the subscripts on $k$ are defined relative to the interface, not relative to scattering plane. Field vectors, of course, may have components in any direction, though the incident wave is assumed to be a standard TEM wave appropriate for the vacuum solution of Maxwell's equation. 
The starting point for the boundary value problem is to assume a form for fields on either side of the boundary. In particular, we consider the possibility (discussed above) that a single incident wave (from vacuum) excites four waves in each dielectric medium. Then one takes the fields to be the following on the upper and lower sides of an interface:

$$
\begin{aligned}
& E_{\text {up }}=\sum_{\alpha=1}^{4} E\left(k_{\alpha}, \omega\right)=\sum_{\alpha=1}^{4} c_{\alpha} \hat{\xi}_{\alpha} \\
& E_{d n}=\sum_{\alpha=5}^{8} E\left(k_{\alpha}, \omega\right)=\sum_{\alpha=5}^{8} c_{\alpha} \hat{\xi}_{\alpha}
\end{aligned}
$$

The second equality in each may be taken as a definition of the unit vectors for the eigenpolarizations, $\hat{\xi}$. These may be complex so the unit vector condition is $\hat{\xi}^{*} \cdot \hat{\xi}=1$. The $k$-dependence has been absorbed into the subscripts and the frequency dependence will be suppressed for the remainder of this discussion.

The boundary conditions for the fields at an interface between two media can just be derived by integrating (4.4) over the usual Gaussian pillbox and Stokesian loop (see, e.g., [Jackson, 1975, 3] pp. 17-20). In this case, one finds B must be continuous accross the interface, as are the components of $\mathbf{D}$ perpendicular to the interface and the components of $\mathbf{E}$ in the plane of the interface. Using the Maxwell equations, (4.4), these conditions reduce to

$$
\begin{gathered}
\mathbf{v} \bullet \sum_{\alpha=1}^{4} c_{\alpha} \hat{\xi}_{\alpha}=\mathbf{v} \bullet \sum_{\alpha=5}^{8} c_{\alpha} \hat{\xi}_{\alpha} \\
\mathbf{w} \bullet \sum_{\alpha=1}^{4} c_{\alpha} \mathbf{k}_{\alpha} \times \hat{\xi}_{\alpha}=\mathbf{w} \bullet \sum_{\alpha=5}^{8} c_{\alpha} \mathbf{k}_{\alpha} \times \hat{\xi}_{\alpha} \\
\mathbf{z} \bullet \sum_{\alpha=1}^{4} c_{\alpha}\left(\mathbf{k}_{\alpha}^{2} \hat{\xi}_{\alpha}-\left(\mathbf{k}_{\alpha} \bullet \hat{\xi}_{\alpha}\right) \mathbf{k}_{\alpha}\right)=\mathbf{z} \bullet \sum_{\alpha=5}^{8} c_{\alpha}\left(\mathbf{k}_{\alpha}^{2} \hat{\xi}_{\alpha}-\left(\mathbf{k}_{\alpha} \bullet \hat{\xi}_{\alpha}\right) \mathbf{k}_{\alpha}\right)
\end{gathered}
$$


where $\mathbf{v}$ is any vector parallel to the interface, $\mathbf{w}$ is any vector at all, and $\mathbf{z}$ is normal to the interface. In particular, these equations correspond, respectively, to the continuity of $\mathbf{E}_{11}, \mathbf{B}$, and $\mathbf{D}_{\mathrm{p}}$. Now it is convenient to introduce the coordinate system shown in Fig. 4.1. Namely, the $z$ direction is chosen to be normal to interface, the $x$ direction is chosen in the plane of the interface and the incident k-vector (the $x z$ plane is the scattering plane) and the $y$ direction is the third right-hand orthonormal direction, as shown. In accordance with the condition above, that all $k$-vectors have the same in plane components and that the $\mathrm{xz}$ plane is the scattering plane, we have

$$
\mathbf{k}_{\alpha}=\mathrm{k}_{\mathrm{II}} \hat{\mathbf{x}}+\mathrm{k}_{\alpha \mathrm{p}} \hat{\mathbf{z}}
$$

Use of the condition (4.31) in equations (4.30) shows that equation (4.30c) and the $z$-component of equation ( $4.30 \mathrm{~b}$ ) are redundant with the others. The independent boundary conditions are that the parallel components of $\mathbf{E}$ and $\mathbf{B}$ are continuous. In component form these are

$$
\begin{aligned}
\sum_{\alpha=1}^{4} c_{\alpha} \xi_{\alpha x} & =\sum_{\alpha=5}^{8} c_{\alpha} \xi_{\alpha x} \\
\sum_{\alpha=1}^{4} c_{\alpha} \xi_{\alpha y} & =\sum_{\alpha=5}^{8} c_{\alpha} \xi_{\alpha y} \\
\sum_{\alpha=1}^{4} c_{\alpha} k_{\alpha p} \xi_{\alpha y} & =\sum_{\alpha=5}^{8} c_{\alpha} k_{\alpha p} \xi_{\alpha y} \\
\sum_{\alpha=1}^{4} c_{\alpha}\left(k_{11} \xi_{\alpha z}-k_{\alpha p} \xi_{\alpha x}\right) & =\sum_{\alpha=5}^{8} c_{\alpha}\left(k_{11} \xi_{\alpha z}-k_{\alpha p} \xi_{\alpha x}\right)
\end{aligned}
$$

The transversality condition, equation (4.18), and the unit magnitude of the polarization vectors also give

$$
\begin{aligned}
& \mathrm{k}_{11} \xi_{\alpha x}+\mathrm{k}_{\alpha \mathrm{p}} \xi_{\alpha z}=0 \\
& \left|\xi_{\alpha \alpha x}\right|^{2}+\left|\xi_{\alpha x y}\right|^{2}+\left|\xi_{\alpha z}\right|^{2}=1
\end{aligned}
$$


which may be solved to remove all components of the polarization but one from equations (4.32).

In particular, using 4.33 to remove $\xi_{\alpha x}$ and the dispersion relation, equation (4.26) equations (4.32) become

$$
\begin{aligned}
& \sum_{\alpha=1}^{4} c_{\alpha} k_{\alpha p} \xi_{\alpha y}=\sum_{\alpha=5}^{8} c_{\alpha} k_{\alpha p} \xi_{\alpha y} \\
& \sum_{\alpha=1}^{4} c_{\alpha} \xi_{\alpha y}=\sum_{\alpha=5}^{8} c_{\alpha} \xi_{\alpha y} \\
& \sum_{\alpha=1}^{4} c_{\alpha} k_{\alpha p} \xi_{\alpha z}=\sum_{\alpha=5}^{8} c_{\alpha} k_{\alpha p} \xi_{\alpha z} \\
& \sum_{\alpha=1}^{4} c_{\alpha} \varepsilon_{\alpha} \xi_{\alpha z}=\sum_{\alpha=5}^{8} c_{\alpha} \varepsilon_{\alpha} \xi_{\alpha z} \\
& k_{\alpha p}= \pm k_{0}\left(\varepsilon\left(k_{\alpha}, \omega\right)-\cos ^{2} \theta\right)^{1 / 2}
\end{aligned}
$$

The signs in (4.36) are chosen so that the various k-vectors are in appropriate directions.

The set of four equations (4.35) relate the eight quantities $c_{\alpha}$ : if any four are specified, the others may be determined. For example if the incident wave field $\left(c_{1}\right.$ and $c_{2}$ ) from the top was specified and the incident wave field from below were known to be zero $\left(c_{7}=c_{8}=0\right)$ then these equations would generate the analog of the Fresnel reflection and transmission coefficients from conventional optics. Alternatively, if the wave fields below an interface were specified, the wave field above the interface could be calculated. In particular it is useful to note that these equation may be cast in matrix form:

$$
\left(\begin{array}{l}
c_{3} \\
c_{4}
\end{array}\right)=\overrightarrow{\mathbf{R}}\left(\begin{array}{l}
c_{1} \\
c_{2}
\end{array}\right)+\overrightarrow{\mathbf{T}}\left(\begin{array}{l}
c_{7} \\
c_{8}
\end{array}\right)
$$




$$
\left(\begin{array}{l}
c_{5} \\
c_{6}
\end{array}\right)=\overrightarrow{\mathbf{T}}\left(\begin{array}{l}
c_{1} \\
c_{2}
\end{array}\right)+\overrightarrow{\mathbf{R}}\left(\begin{array}{l}
c_{7} \\
c_{8}
\end{array}\right)
$$

where the components of the matrices are determined from (4.35). The analogy with the usual Fresnel coefficients is clear: $\overrightarrow{\mathbf{R}}$ and $\overrightarrow{\mathbf{T}}$ are the reflectivity and transmission matrices for waves incident from the top of the interface (assuming no upward propagating waves in the lower medium) while the primed quantities are for waves incident from below the interface. Exact expressions for the components of these matrices may be written down from equations (4.35) but they are cumbersome and, except in special cases, the equations are solved numerically.

\section{Isotropic Media}

Equations (4.35) simplify in the case of isotropic media. In each medium, $\varepsilon$ is independent of the wave field under consideration, and likewise, so is $\mathrm{k}$ (up to the sign). In addition, the eigenpolarizations may be chosen to be perpendicular and parallel to the $x z$ plane, typically called sigma and pi polarizations, respectively. Under these conditions, equations (4.35a) and (4.35b) are independent of ( $4.35 \mathrm{c})$ and (4.35d), or, equivalently, all matrices in (4.37) are diagonal. Taking wave fields $1,3,5$ and 7 to be sigma polarized $\left(\xi_{y}=1, \xi_{x}=\xi_{z}=0\right)$ and $2,4,6$ and 8 to be pi polarized ( $\left.\xi_{y}=0, \xi_{x}=k_{p} / k, \xi_{z}=-k_{11} / k\right)$, and primed quantities to be in the lower medium (un-primed in the upper), one has

$$
\begin{aligned}
& c_{1}+c_{3}=c_{5}+c_{7} \\
& k_{p}\left(c_{1}-c_{3}\right)=k_{p}^{\prime}\left(c_{5}-c_{7}\right) \\
& \varepsilon \xi_{z}\left(c_{2}+c_{4}\right)=\varepsilon^{\prime} \xi_{z}^{\prime}\left(c_{6}+c_{8}\right) \\
& k_{p} \xi_{z}\left(c_{2}-c_{4}\right)=k_{p}^{\prime} \xi_{z}^{\prime}\left(c_{6}-c_{8}\right)
\end{aligned}
$$

Direct solution of these in the special case where $c_{7}$ and $c_{8}$ are zero gives the standard Fresnel coefficients for reflection and transmission. In particular, 


$$
\begin{aligned}
& \mathrm{R}_{\sigma}=\frac{\mathrm{c}_{3}}{\mathrm{c}_{1}}=\frac{\mathrm{k}_{\mathrm{p}}-\mathrm{k}_{\mathrm{p}}^{\prime}}{\mathrm{k}_{\mathrm{p}}+\mathrm{k}_{\mathrm{p}}^{\prime}} \\
& \mathrm{T}_{\sigma}=\frac{\mathrm{c}_{5}}{\mathrm{c}_{1}}=\frac{2 \mathrm{k}_{\mathrm{p}}}{\mathrm{k}_{\mathrm{p}}+\mathrm{k}_{\mathrm{p}}^{\prime}} \\
& \mathrm{R}_{\pi}=\frac{\mathrm{c}_{4}}{\mathrm{c}_{2}}=\frac{\mathrm{k}_{\mathrm{p}} / \varepsilon-\mathrm{k}_{\mathrm{p}}^{\prime} / \varepsilon^{\prime}}{\mathrm{k}_{\mathrm{p}} / \varepsilon+\mathrm{k}_{\mathrm{p}}^{\prime} / \varepsilon^{\prime}} \\
& \mathrm{T}_{\pi}=\frac{\mathrm{c}_{6}}{\mathrm{c}_{2}}=\frac{\mathrm{k}^{\prime}}{\mathrm{k} \frac{\varepsilon^{\prime}}{\varepsilon} \mathrm{k}_{\mathrm{p}}+\mathrm{k}_{\mathrm{p}}^{\prime}} \\
& \mathrm{k}_{\mathrm{p}} \equiv-\left(\varepsilon-\cos ^{2} \theta\right)^{1 / 2}
\end{aligned}
$$

Where $k$ and $k$ ' are the magnitudes of the appropriate k-vectors. In fact, the factor $k^{\prime} / \mathrm{k}$ in the last equation is nearly one and may be dropped. In addition, for most of the following we will be concerned with grazing incidence. In this case, the differences between $k_{p}$ and $k_{p}$ ' are larger than the changes in the dielectric constants, so that one may take $\varepsilon=\varepsilon^{\prime}$ in both equations (4.42c) and (4.42d). The reflectivities for sigma and pi radiation in this case are degenerate, so that one may write

$$
\begin{array}{cc}
\mathrm{R}\left(\theta, \varepsilon, \varepsilon^{\prime}\right)=\frac{\mathrm{k}_{\mathrm{p}}-\mathrm{k}_{\mathrm{p}}{ }^{\prime}}{\mathrm{k}_{\mathrm{p}}+\mathrm{k}_{\mathrm{p}}{ }^{\prime}}=\frac{1-\beta}{1+\beta} & \left(\begin{array}{l}
\text { Isotropic } \\
\text { Material }
\end{array}\right) \\
\mathrm{T}\left(\theta, \varepsilon, \varepsilon^{\prime}\right)=\frac{2 \mathrm{k}_{\mathrm{p}}}{\mathrm{k}_{\mathrm{p}}+\mathrm{k}_{\mathrm{p}}{ }^{\prime}}=\frac{2}{1+\beta} \quad\left(\begin{array}{l}
\text { Isotropic } \\
\text { Material }
\end{array}\right) \\
\beta \equiv \frac{\mathrm{k}_{\mathrm{p}}}{\mathrm{k}_{\mathrm{p}}} \approx \frac{\left[\theta^{2}-2 \delta^{\prime}\right]^{1 / 2}}{\left[\theta^{2}-2 \delta\right]^{1 / 2}}
\end{array}
$$

The approximate equality in (4.45) has been obtained by a small angle expansion and assuming that the form of $\varepsilon^{\prime}$ is

$$
\varepsilon \equiv \mathrm{n}^{2}=(1-\delta)^{2} \approx 1-2 \delta
$$


where $\mathrm{n}$ is the index of refraction of the material and $\delta<<1$. Another useful relationship that follows directly from (4.44) (or from the continuity of the in plane component of the $E$ field) is that

$$
T=1+R
$$

We also note that the other quantities in equation (4.37) are easily seen to be $R^{\prime}=-R=(\beta-1) /(\beta+1), T^{\prime}=1+R^{\prime}=1-R=2 \beta /(\beta+1)$.

We consider the case of $14.413 \mathrm{keV} x$-rays incident (from vacuum) onto a thick layer of bulk density iron. One has $\delta=0$ and $\delta^{\prime}=7.4 \times 10^{-6}+i 3.4 \times 10^{-7}$ [Cromer and Liberman, 1981, 13] [Brennan and Cowan, 1992, 16] Figure 4.3 shows both the reflectivity, $|R|^{2}$ and the transmission $|T|^{2}$ as a function of grazing angle. Total external reflection is evident for small angles, with a swift fall off in reflectivity at angles of incidence above the critical angle, $\theta_{c}=(2 \operatorname{Re}\{\delta\})^{1 / 2}=3.8$ mrad. The transmission (really the field intensity at the surface) peaks at the critical angle, where both reflected waves and incident waves are in phase and large. Note that, for this case, the approximations used to go from (4.42) to (4.44), (the small angle

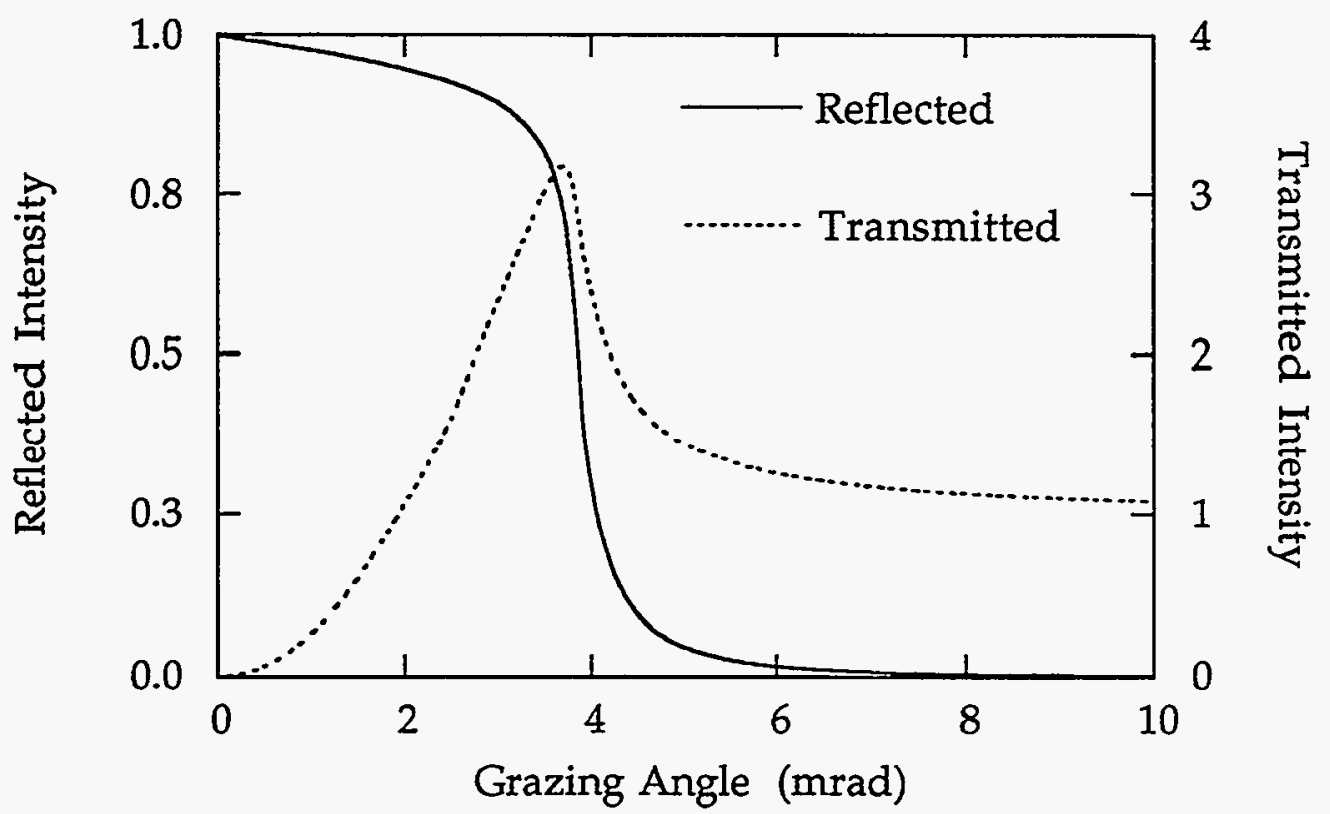

Figure 4.2. Fresnel reflection and transmission coefficients for bulk density iron at $14.4 \mathrm{keV}$. 
approximation and assuming $\mathrm{pi}=$ sigma), introduce fractional errors of less than $5 \times 10^{-4}$ in the calculation $|R|^{2}$ and $|T|^{2}$ out to an angle of $50 \mathrm{mrad}$. Thus (4.44) is a very good approximation.

\section{Reflectivity from a Resonant Medium.}

In going from the isotropic electronic reflectivity of materials to the reflectivity of a nuclear resonant material, there are two significant changes. First, the resonant nature of the nuclear transition means that the reflectivity is sharply energy dependent, and second, there can be complicated polarization effects. For the moment, we focus on the energy dependence. This was first investigated in the papers of Bernstein and Campbell [Bernstein and Campbell, 1963, 17] and of Wagner [Wagner, 1968, 18].

Mathematically, the reflectivity for a single isolated transition (without complicated polarization dependence) can be handled exactly as the isotropic case, except that the frequency dependence of the scattering amplitude (or delta) must be included explicitly. One has, for $\mathrm{x}$-rays incident from vacuum onto a material with an isotropic resonant transition

$$
\begin{aligned}
& \mathrm{R}(\theta, \omega)=\frac{1-\beta}{1+\beta} \\
& \beta=\left[1-2 \delta(\omega) / \theta^{2}\right]^{1 / 2}
\end{aligned}
$$

where one takes $\delta(\omega)=\delta_{\mathrm{e}}+\delta_{\mathrm{n}}(\omega)$. $\delta_{\mathrm{e}}$ is due to electronic scattering and may be taken as frequency independent $t^{*}$ (relative to the nuclear response) while $\delta_{n}(\omega)$ is the nuclear part and has a Lorentzian frequency dependence. $\delta(\omega)$ is plotted in figure 4.3.

\footnotetext{
* In all experiments discussed in this thesis, the bandwidth of the incident radiation is sufficiently small so that the frequency (energy) dependence of the electronic scattering may be neglected.
} 


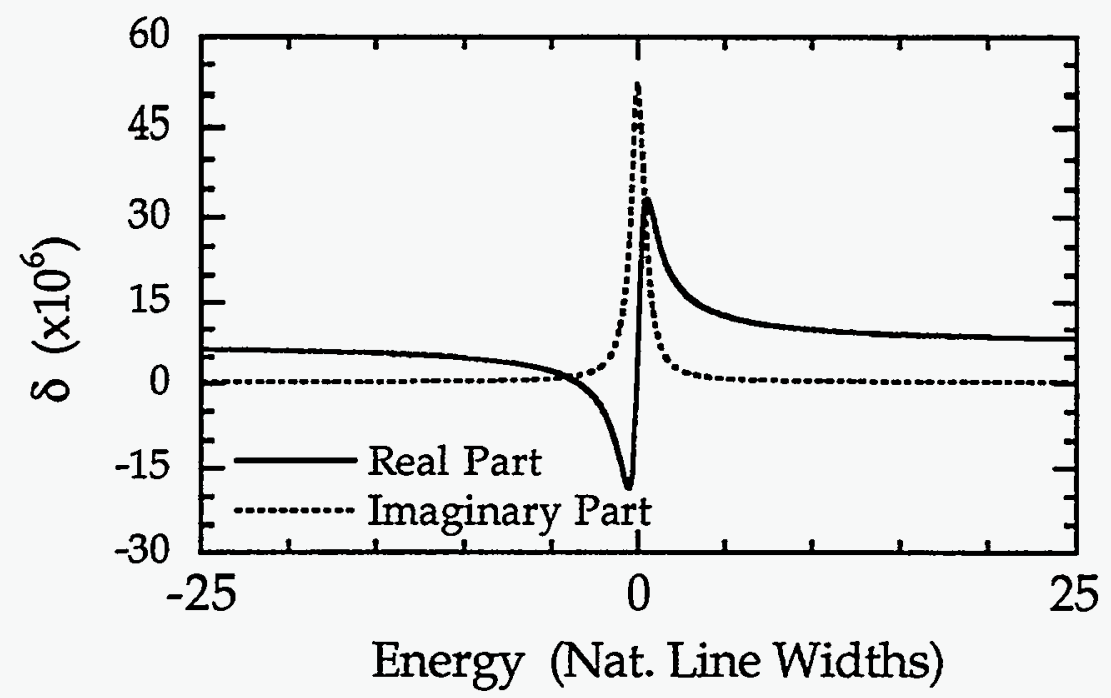

Figure 4.3. Index of refraction for bulk density ${ }^{57} \mathrm{Fe}$ without hyperfine splitting (resonant fraction-enrichment product $=0.35$ ). The real part (solid line) and magnitude of the imaginary part (dashed line) are both shown.

In general, the resonant contribution has three effects: it increases delta at frequencies above the resonant transition, decreases delta below the transition and adds an imaginary part to delta in the immediate neighborhood of the transition. Thus, up to the effect of increased absorption, one expects the reflectivity at a given angle to be enhanced relative to the electronic reflectivity at frequencies above the transition frequency, and decreased at frequencies below the resonance. Which effect is most visible is dependent on the angle of incidence, as is shown in figure 4.4. Below the critical angle the reflectivity is nearly saturated and the effect of the resonance is mostly to reduce the reflectivity at energies below the transition energy (in effect, the reflection below the critical angle with a resonant transition acts as a band reject filter on the incident radiation). Above the critical angle, the background reflectivity due to electronic scattering is small so the resonance primarily enhances the reflectivity at frequencies above the critical angle (a band-pass filter). In the immediate neighborhood of the critical angle, the reflectivity is very sensitive to changes in delta, and thus the resonance perturbs the electronic response even when the energy is far from the resonance value. 


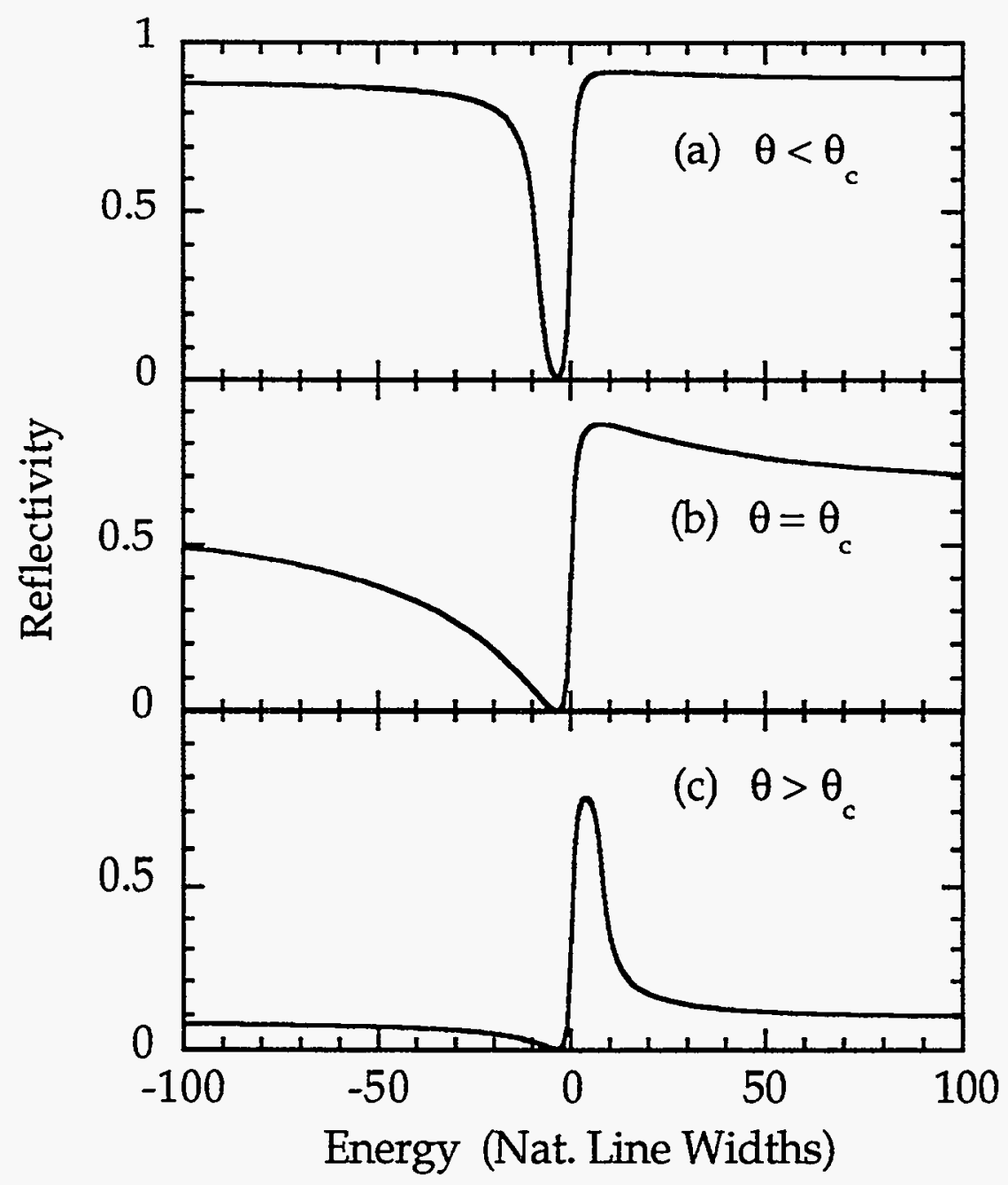

Figure 4.4. Reflectivity in the vicinity of an unsplit nuclear transition for the angle of incidence (a) below the critical angle, (b) at the critical angle and (c) above the critical angle (3.0, 3.8 and 4.6 mrad, respectively). The index of refraction is as given in figure 4.3.

\section{Polarization Effects}

Resonant transitions may affect the polarization properties of the reflected beam. In particular, as discussed in appendix $A$, the eigenpolarizations within a resonant medium are not generally the simple sigma and pi polarizations typically used to describe scattering from isotropic materials. We will be concerned with two cases, scattering from iron which has its quantization axis either perpendicular to the incident photon direction, or parallel to it. In the 
former case, the polarizations are indeed the sigma and pi polarizations. The reflectivity as a function of energy (for $\theta>\theta_{C}$ ) is shown in figure 4.5.

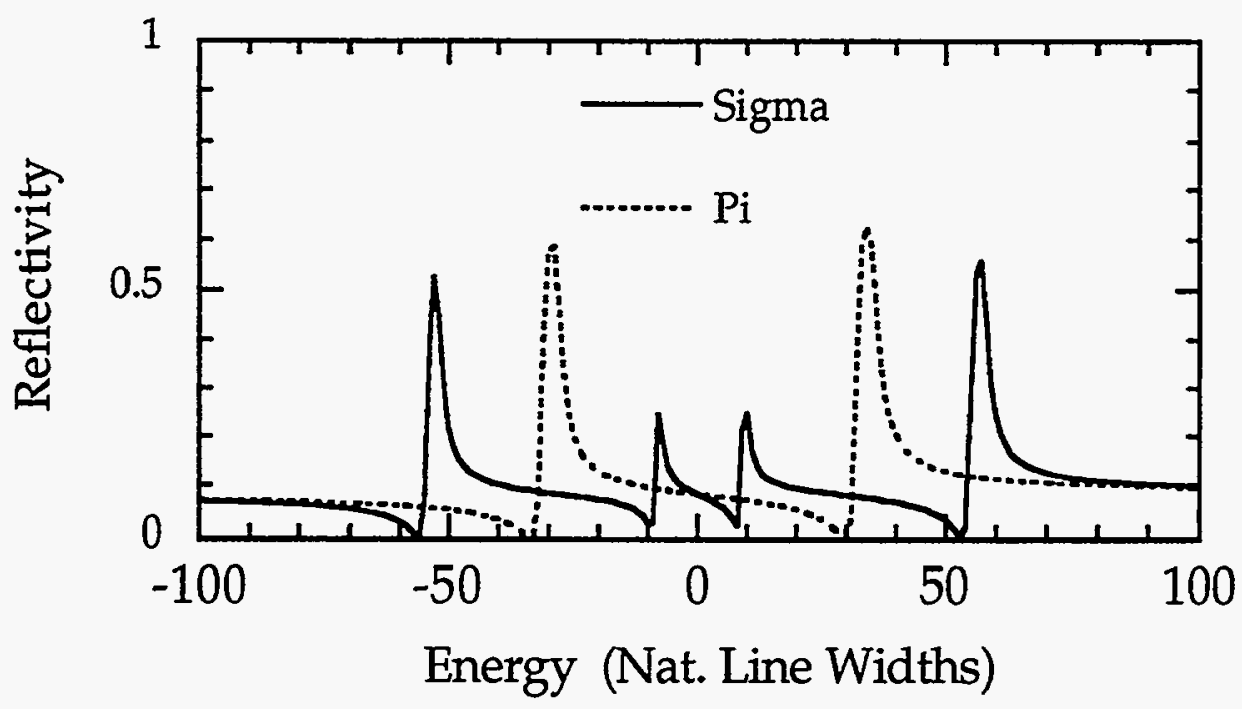

Figure 4.5. Reflectivity from hyperfine split iron of bulk density with a magnetic field in the plane of the interface and perpendicular to the photon propagation direction. Resonant fraction-enrichment product $=0.35$.

In the case where the quantization axis is parallel to the incident beam, the internal polarizations are circular, so that the reflectivity for sigma and $\mathrm{pi}$ polarized light is not well defined: the matrix $R$ is not diagonal in a sigma-pi basis. However, the reflectivity for incident circularly polarized light is well defined and this is plotted, again for typical iron, in figure 4.7. 


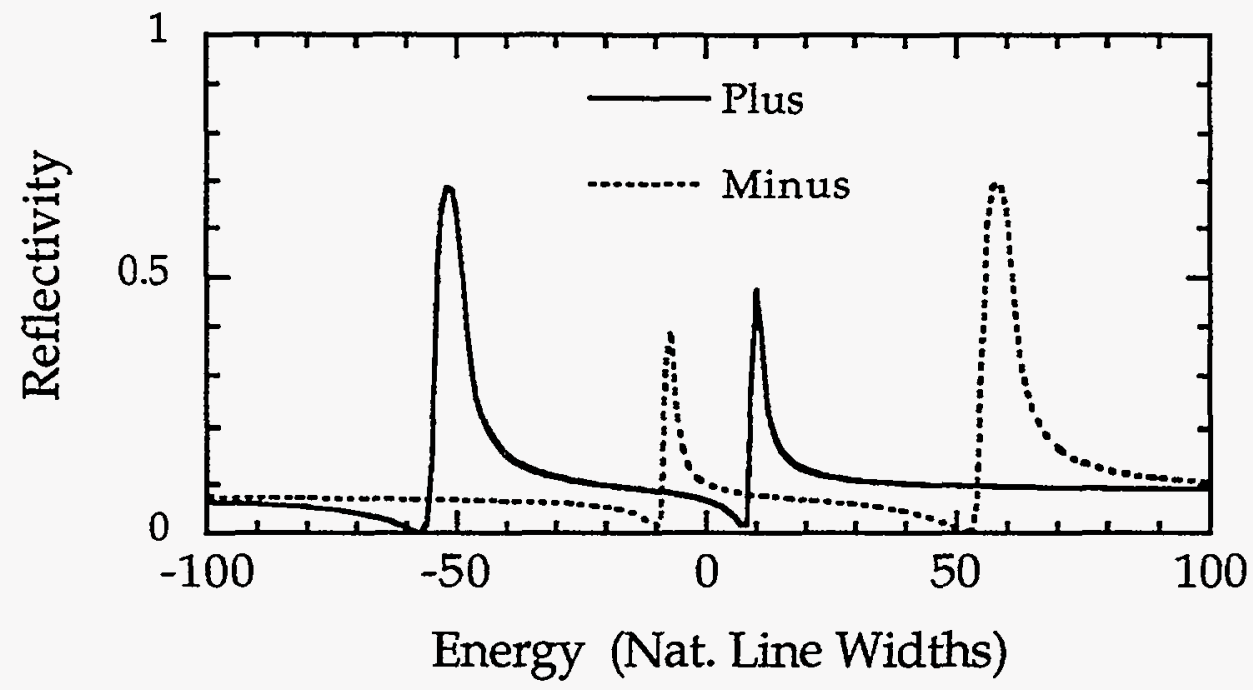

Figure 4.6. Reflectivity from hyperfine split iron of bulk density with a magnetic field in the plane of the interface and parallel to the photon propagation direction. Resonant fraction-enrichment product $=0.35$.

\section{Comparison with the GIAR Theory}

The results (4.35) may be reduced to those of Hannon et al [Hannon, et al., $1985,1]$ for grazing incidence anti-reflection (GIAR) films. In particular, the work in the series of papers describing GIAR films [Hannon, et al., 1985, 19, Hannon, et al., 1985, 20, Hannon, et al., 1985, 1, Hannon, et al., 1985, 21] is for the special case when the angle of incidence is small and there is only one anisotropic layer in a multilayer stack. This allows several useful approximations to be made. The small angle of incidence (and therefore small scattering angle) allows one to neglect the differences in the eigenpolarizations and index of refraction between the upward and downward propagating waves in the anisotropic layer: the layer is assumed to have only two independent wave-fields, instead of the more general case of four used to generate (4.35). One also takes the eigenpolarizations in all layers to be those of the single anisotropic layer and neglects the differences in polarization caused by the refraction of the waves (this is equivalent to neglecting the difference between sigma and pi reflectivities in the isotropic case described above). 
Returning to (4.35) and taking the top layer to be isotropic, and the lower layer to be the anisotropic one, these assumptions have the following consequences:

$$
\begin{array}{cc}
k_{\mathrm{p} 1}=\mathrm{k}_{\mathrm{p} 2}=-\mathrm{k}_{\mathrm{p} 3}=-\mathrm{k}_{\mathrm{p} 4} & \mathrm{k}_{\mathrm{p} 7}=-\mathrm{k}_{\mathrm{p} 3}, \mathrm{k}_{\mathrm{p} 8}=-\mathrm{k}_{\mathrm{p} 6}, \\
\xi_{1 \mathrm{y}}=\xi_{3 \mathrm{y}}=\xi_{5 \mathrm{y}}=\xi_{7 \mathrm{y}} & \xi_{1 \mathrm{z}}=\xi_{3 \mathrm{z}}=\xi_{5 \mathrm{z}}=\xi_{7 \mathrm{z}} \\
\xi_{2 \mathrm{y}}=\xi_{4 \mathrm{y}}=\xi_{6 \mathrm{y}}=\xi_{8} & \xi_{2 \mathrm{z}}=\xi_{4 \mathrm{z}}=\xi_{6 \mathrm{z}}=\xi_{8 \mathrm{z}} .
\end{array}
$$

In addition, we ignore the difference of the dielectric constants from one in (4.35d). Then, assuming independent excitation of the eigenpolarizations, equations (4.35) reduce to the same equations as were derived for the sigma reflectivity of isotropic layers. Thus the procedure suggested in the GIAR papers is just to divide the incident wave into the eigenpolarizations of the anisotropic layer and then solve the problem for each polarization component independently.

The results of the GIAR theory have been checked against those of the full theory (equations 4.35) and good agreement has been found in cases where the GIAR theory applies.

\section{Recursive Solution to the Multilayer Problem.}

Equations (4.35) or (4.37) allow the problem of a wave incident on a multilayer structure (fig. 4.1) to be solved recursively. Starting at the bottom interface, one uses (4.35) to generate a matrix relating $\left(c_{3}, c_{4}\right)$ to $\left(c_{1}, c_{2}\right)$ assuming there is no upwardly propagating wave in the lower material $\left(c_{7}=c_{8}=0\right)$. Then, since the propagation of the wave in each medium is understood, one may convert the matrix relation between the fields at the lower interface into a relationship between the fields just below the next interface. Equations (4.35) are then used at this next interface and the process continues until one has a matrix relating the incident and reflected waves at the top interface. 
In a little more detail, one proceeds as follows. Take $c_{1}-c_{8}$ to be the relative field amplitudes at the lower interface (see fig 4.7) and $\tilde{c}_{1}-\tilde{c}_{8}$ to be the relative amplitudes at the upper interface.

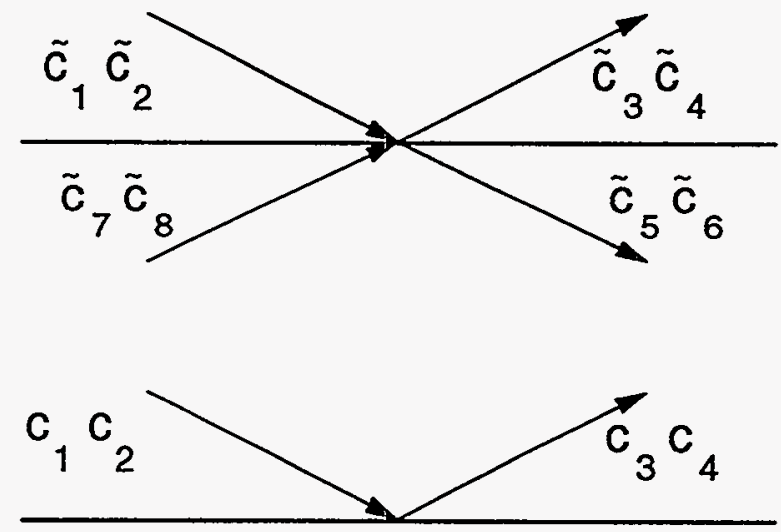

Figure 4.7. Wave fields for the recursive solution to the multilayer problem.

Our problem reduces to determining a relationship between $\left(\tilde{\mathrm{c}}_{1}, \tilde{\mathrm{c}}_{2}\right)$ and $\left(\tilde{\mathrm{c}}_{3}, \tilde{\mathrm{c}}_{4}\right)$ given the relationship between $\left(c_{1}, c_{2}\right)$ and $\left(c_{3}, c_{4}\right)$ : i.e. given $\overrightarrow{\mathbf{R}}_{\text {lower }}$ find $\overrightarrow{\mathbf{R}}_{\text {upper }}$, where these matrices are defined by

$$
\begin{aligned}
& \left(\begin{array}{c}
c_{3} \\
c_{4}
\end{array}\right)=\overrightarrow{\mathbf{R}}_{\text {lower }}\left(\begin{array}{c}
c_{1} \\
c_{2}
\end{array}\right) \\
& \left(\begin{array}{c}
\tilde{c}_{3} \\
\tilde{c}_{4}
\end{array}\right)=\overrightarrow{\mathbf{R}}_{\text {upper }}\left(\begin{array}{c}
\tilde{c}_{1} \\
\tilde{c}_{2}
\end{array}\right)
\end{aligned}
$$

The form of the field in the layer gives

$$
\begin{aligned}
& \left(\begin{array}{l}
\tilde{c}_{5} \\
\tilde{c}_{6}
\end{array}\right)=\left(\begin{array}{cc}
e^{+i k_{1 p} \mathrm{~d}} & 0 \\
0 & \mathrm{e}^{+\mathrm{ik} k_{2 \mathrm{p}} \mathrm{d}}
\end{array}\right)\left(\begin{array}{l}
c_{1} \\
c_{2}
\end{array}\right) \equiv \overrightarrow{\mathbf{P}}_{1}\left(\begin{array}{l}
c_{1} \\
c_{2}
\end{array}\right) \\
& \left(\begin{array}{l}
\tilde{c}_{7} \\
\tilde{c}_{8}
\end{array}\right)=\left(\begin{array}{cc}
\mathrm{e}^{+\mathrm{ik} k_{3 \mathrm{p}} \mathrm{d}} & 0 \\
0 & \mathrm{e}^{+i \mathrm{k}_{4 \mathrm{p}} \mathrm{d}}
\end{array}\right)\left(\begin{array}{l}
c_{3} \\
c_{4}
\end{array}\right) \equiv \overrightarrow{\mathbf{P}}_{2}\left(\begin{array}{l}
c_{3} \\
c_{4}
\end{array}\right)
\end{aligned}
$$


Where $d$ is the thickness of the layer. Substitution of (4.50) and (4.51) into (4.37) then allows $\overrightarrow{\mathbf{R}}_{\text {upper }}$ to be expressed in terms of $\overrightarrow{\mathbf{R}}_{\text {lower }}$ and the matrices $\overrightarrow{\mathbf{R}}, \overrightarrow{\mathbf{T}}, \overrightarrow{\mathbf{R}}$ ' and $\overrightarrow{\mathbf{T}}^{\prime}$, all at the upper interface, and the propagation matrices, $\overrightarrow{\mathbf{P}}_{1}$ and $\overrightarrow{\mathbf{P}}_{2}$. Carrying out the algebra gives

$$
\overrightarrow{\mathbf{R}}_{\text {upper }}=\overrightarrow{\mathbf{R}}+\overrightarrow{\mathbf{T}}^{\prime} \overrightarrow{\mathbf{P}}_{2} \overrightarrow{\mathbf{R}}_{\text {lower }}\left(\overrightarrow{\mathbf{P}}_{1}-\overrightarrow{\mathbf{R}}^{\prime} \overrightarrow{\mathbf{P}}_{2} \overrightarrow{\mathbf{R}}_{\text {lower }}\right)^{-1} \overrightarrow{\mathbf{T}}
$$

Assuming isotropic materials in the recursion relation, (4.52), gives

$$
\begin{aligned}
R_{\text {upper }} & =\frac{R+\left(T T^{\prime}-R R^{\prime}\right) e^{-2 i k_{1 p} d} R_{\text {lower }}}{1-e^{-2 i k_{1 p}^{d}} R^{\prime} R_{\text {lower }}} \\
& =\frac{R+e^{-2 i k_{1 p} d} R_{\text {lower }}}{1+e^{-2 i k_{1 p^{d}}} R_{\text {lower }} R} \quad\left(\begin{array}{c}
\text { Isoptropic } \\
\text { Material }
\end{array}\right)
\end{aligned}
$$

where $R$ is the Fresnel reflectivity of the upper interface, $R_{\text {lower }}$ is the reflectivity at the lower interface (including modification for all lower layers) and $R_{\text {upper }}$ is the total reflectivity of the upper interface. Note that equation 4.47 and the forms for $T^{\prime}$ and $\mathrm{R}^{\prime}$ (see paragraph below 4.47 ) have been used in deriving (4.53b) Strictly speaking, these equations only apply for sigma radiation, but, at grazing incidence, they can be taken as correct for pi radiation as well (see above).

Comparison with the theory of Irkaev, et al.

Recently Irkaev et al [Irkaev, et al., 1993, 2] have presented a different approach to grazing incidence calculations. The starting point is the same Maxwell equations as were used here, but cast in a matrix formalism that has been used with visible light and ellipsometry (see [Azzam and Bashra, 1977, 22], pp. 340-347). The one-dimensional nature of the problem (i.e.: variation in material properties only in the $\mathrm{z}$ direction) allows the solution to be stated as a series of matrices describing the propagation of the fields in each layer, or more exactly, relating the in plane components of $\mathrm{E}$ and $\mathrm{H}$ at the boundaries of each layer (recall these were the only independent components in solving the 
boundary value problem). The results should therefore be identical to those given here, with a repetitive method allowing the solution of multilayer problems. One notes however, that the results of Irkaev et al, are directly in terms of the fields in each layer and thus, while they do not yield the reflectivity so directly as the method described above, they may be more convenient for finding the fields as a function of depth in the sample (though, with a little manipulation, either method can provide either quantity.)

\section{References for Chapter 4}

1 J.P. Hannon, G.T. Trammell, M. Mueller, E. Gerdau, R. Rüffer, and H. Winkler, Grazing-Incidence Antireflection films III: General theory for pure nuclear reflections. Phys. Rev. B 32 (1985) 6363.

2 S.M. Irkaev, M.A. Andreeva, V.G. Semenov, G.N. Belozerskii, and O.V. Grishin, Grazing incidence Mössbauer spectroscopy: new method for surface layers analysis. Part II. Theory of grazing incidence Mössbauer spectra. Nuclear Instruments and Methods in Physics Research B74 (1993) 554.

3 J.D. Jackson, Classical Electrodynamics. New York: John Wiley and Sons (1975).

4 L.D. Landau, E.M. Lifshitz, and L.P. Pitaevskii, Electrodynamics of Continuous Media. Second Revised Edition ed. New York: Pergamon Press (1984).

5 V.M. Agranovich and V.L. Ginzburg, Spatial Dispersion in Crystal Optics and the Theory of Excitons. New York: Interscience (1966).

6 V.L. Ginzburg, A.A. Rukhadze, and V.P. Silin, The Electrodynamics of Crystals and the Theory of Excitons. Journal of Physical Chemisrty of Solids 23 (1962) 85.

7 M. Lax, Multiple Scattering of Waves. Rev. Mod. Phys. 23 (1951) 287.

8 M.L. Goldberger and K.M. Watson, Collision Theory. New York: Reprinted in 1975 by R.E. Krieger, Publishing Co., Inc. (1964).

9 J.J. Sakurai, Advanced Quantum Mechanics. London: Addison-Wesley (1967). 
10 J.P. Hannon and G.T. Trammell, Mössbauer Diffraction. II. Dynamical Theory of Mössbauer Optics. Phys. Rev. 186 (1969) 306.

11 R.W. James, The Optical Principles of the Diffraction of X-rays. Woodbridge, Connecticut: Ox Bow Press (1962 (Reprinted 1982)).

12 W.H. McMaster, N.K.D. Grande, J.H. Mallet, and J.H. Hubbell, Compilation of $X$-ray Cross Sections. Lawrence Radiation Laboratory, Rep. No. UCRL50174 Sec. III Rev1 (1969).

13 D.T. Cromer and D.A. Liberman, Acta Crystallogr. A37 (1981) 267.

14 B.L. Henke, E.M. Gullikson, and J.C. Davis, X-ray interactions: photoabsorption, scattering, transmission and reflection. Atomic Data and Nuclear Data Tables 54 (1992) 181.

15 M. Born and E. Wolf, Principles of Optics. Sixth Edition ed. New York: Pergamon Press (1980).

16 S. Brennan and P.L. Cowan, $A$ suite of programs for calculating $x$-ray absorption, reflection and diffraction performance for a variety of materials at arbitrary wavelengths. Rev. Sci. Instrumen. 63 (1992) 850.

17 S. Bernstein and E.C. Campbell, Nuclear Anomalous Dispersion in Fe57 by the Method of Total Reflection. Phys. Rev. 132 (1963) 1625.

18 F.E. Wagner, Totalreflexion der rückstossfreien $8.4 \mathrm{keV}$ g-Strahlung des Tm169. Zeitscrift für Physic 210 (1968) 361.

19 J.P. Hannon, N.V. Hung, G.T. Trammell, E. Gerdau, M. Mueller, R. Rüffer, and H. Winkler, Grazing-incidence Antireflection films I: Basic theory. Phys. Rev. B 32 (1985) 5068.

20 J.P. Hannon, N.V. Hung, G.T. Trammell, E. Gerdau, M. Mueller, R. Rüffer, and $\mathrm{H}$. Winkler, Grazing-incidence Antireflection films II: Alternate techniques and General multilayer theory. Phys. Rev. B 32 (1985) 5081.

21 J.P. Hannon, G.T. Trammell, M. Mueller, E. Gerdau, R. Rüffer, and H. Winkler, Grazing-Incidence Antireflection Films. IV. Application to Mössbauer Filtering of Synchrotron Radiation. Phys. Rev. B32 (1985) 6374.

22 R.M.A. Azzam and N.M. Bashra, Ellipsometry and Polarized Light. New York: North Holland Publishing Co. (1977). 


\section{Rough Interfaces.}

\section{Introduction}

The previous chapter developed theory appropriate for describing the scattering that occurs at ideal smooth interfaces in multilayer structures. In reality, interfaces are not flat and it is well known that deviations from ideal structure, roughness, can have substantial effects on the reflectivity measured from layered materials. Therefore it is important that the theory described above be modified to include the roughness that will be present at real interfaces.

Deviations from ideal interface structure can be grouped into two broad categories: rough interfaces and graded interfaces. The basic idea is shown in figure 5.1 .

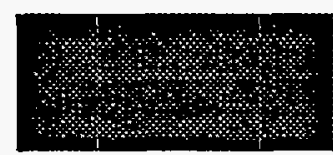

(a)

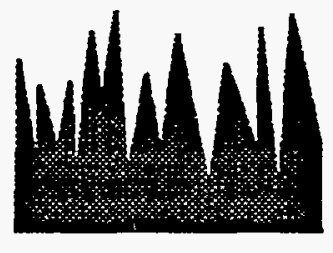

(b)

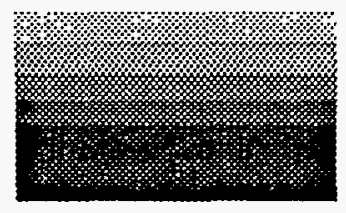

(c)

Figure 5.1. Different types of idealized interfaces: (a) flat, (b) purely rough and (c) graded.

Figure 5.1a shows an ideal interface between two materials, the type for which the solution of the previous chapter is appropriate. Figure $5.1 \mathrm{~b}$ shows a purely rough interface. In this case, there are still two distinct regions, one of each type of material, but the boundary between them is not flat. This means that there will some be diffuse scattering into non-specular directions. Figure $5.1 \mathrm{c}$ shows a graded interface. In this case there is only mixing or inter-diffusion between the two layers, and this inter-diffusion is assumed uniform in the plane of the interface. All scattering from a graded interface will preserve the specular condition; there will be no diffuse scattering from a graded interface. 
A real interface will, in general, be some combination of rough and graded and the tendency toward one or the other type will have strong influence on the amount of diffuse scattering from the interface. However, we are only concerned with specular scattering, and, in this case the two type of interfaces are essentially indistinguishable. This is because $x$-ray scattering probes the Fourier components of the scattering amplitude (or charge distribution for simple electronic scattering) in the direction of the momentum change of the photon. Specular scattering means the momentum transfer is purely perpendicular to the surface, and so it probes only the average density as a function of depth. Therefore it is not possible distinguish between a rough and a graded interface just by measuring the intensity of a specularly reflected beam.

Strictly speaking, the above statement is true only if the transverse scale of the roughness is small compared to the size of the first Fresnel zone (see the discussion to follow) and if the angle of incidence is not so small that multiple scattering of $\mathrm{x}$-rays within the interface must be considered (see [Smirnov, et al., $1979,1])$. However, the former condition seems to be met in most cases, and the latter should only be a concern at extreme grazing angles ( $<1 \mathrm{mrad}$ ) which are not investigated in this work. Thus, for the calculation of specular reflection, all non-ideal characteristics of a rough interface may be replaced by a graded interface, and the response may then be calculated by the methods of the previous chapter (one just divides up the interface into thin layers having uniform response).

The following discussion has three sections. First there a kinematic approximation that shows the importance of the transverse scale of the roughness relative to the Fresnel zone size. Then a distorted wave Born approximation (DWBA) is introduced to allow for dynamical interactions, with the details of the calculation presented in appendix $C$. The DWBA allows introduction of a fairly simple roughness correction that may be used for calculating the specular response of rough multilayers. However, this correction is only an approximation, and, given that we can reduce a rough interface to a graded one (for specular scattering) the accuracy of the correction can be evaluated by dividing up the interface into may small regions of uniform response and doing the right calculation as outlined in chapter 4. It turns out 
that the roughness correction is actually quite good at describing the response of electronic materials, but, some care must be taken when the imaginary part of the index of refraction is large, as can happen in the vicinity of resonant transitions.

\section{Kinematic Scattering}

A kinematic approximation relies on the assumptions that the scattering by the individual constituents of a system is weak and that the total scattered field should be very small relative to the incident field. Note that both conditions must be satisfied and are different: while $x$-ray scattering by atoms or nuclei is always weak, it is possible to arrange things so that many scatterers will add in phase (i.e. Bragg reflections from thick crystals or total external reflection) so that the scattered wave will be large. In particular, for a kinematic approximation, one assumes that every object in the scattering system sees the same incident wave, and that wave is exactly the wave that would be present if there were no scatterers present, i.e. the vacuum wave. This is analogous to the case of the Born approximation in quantum mechanics.

The incident wave is taken to have the form ${ }^{*}$

$$
E_{i}(x)=E_{i} \hat{\mathbf{e}}_{i} e^{+i k_{i} \bullet x}
$$

The (far) field at position $\mathbf{x}^{\prime}$ with polarization $\hat{\mathbf{e}}_{f}$ due to a scatterer at position $\mathbf{x}$ is

$$
E_{f}\left(x^{\prime}\right)=E_{i} e^{+i k_{i} \bullet x} \frac{e^{+i k_{0}\left|x^{\prime}-x\right|}}{\left|x-x^{\prime}\right|} \hat{\mathbf{e}}_{f}^{*} \vec{F}\left(\mathbf{k}_{i}, \tilde{k}_{f}, \omega\right) \hat{\mathbf{e}}_{i}
$$

where $\overrightarrow{\mathbf{F}}$ is the scattering amplitude of the particle and

$$
\tilde{\mathbf{k}}_{\mathrm{f}} \equiv\left|\mathbf{k}_{\mathrm{i}}\right| \frac{\mathbf{x}^{\prime}-\mathbf{x}}{\left|\mathbf{x}^{\prime}-\mathbf{x}\right|}=\mathrm{k}_{0} \frac{\mathbf{x}^{\prime}-\mathbf{x}}{\left|\mathbf{x}^{\prime}-\mathbf{x}\right|}
$$

$\mathrm{k}_{0}=\omega / \mathrm{c}$ is the magnitude of the free space wave vector. Integrating this over some number distribution, $N(x)$, of scatterers, one has

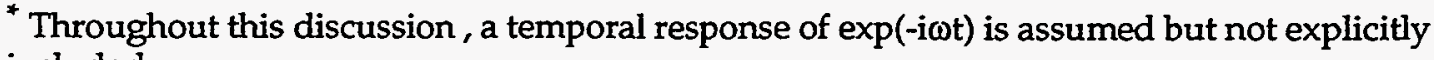
included.
} 


$$
\frac{E_{f}\left(x^{\prime}\right)}{E_{i}}=\int \frac{\hat{\mathbf{e}}_{f}^{*} \vec{F}\left(\mathbf{k}_{i}, \tilde{\mathbf{k}}_{f}, \omega\right) \hat{\mathbf{e}}_{i}}{\left|x-x^{\prime}\right|} e^{+i k_{0}\left|x-x^{\prime}\right|} e^{+i \mathbf{k}_{i} \bullet x} N(x) d^{3} x
$$

The dimensions of the scatterer are assumed small compared with the distance to the observation point, and the origin is taken to lie within the scatterer so that $|\mathbf{x}|<\left|\mathbf{x}^{\prime}\right|$. Defining $r=\left|\mathbf{x}^{\prime}\right|$ expansion to second order gives

$$
\begin{aligned}
k_{0}\left|x-x^{\prime}\right| & =k_{0} r\left(1+\frac{|x|^{2}}{r^{2}}-2 \frac{x \cdot x^{\prime}}{r^{2}}\right)^{1 / 2} \\
& \approx k_{0} r-k_{f} \cdot x+\frac{k_{0}}{2 r}\left(|x|^{2}-\frac{1}{k_{0}{ }^{2}}\left(k_{f} \bullet x\right)^{2}\right)
\end{aligned}
$$

where

$$
\mathbf{k}_{\mathrm{f}} \equiv \mathrm{k}_{0} \frac{\mathbf{x}^{\prime}}{\left|\mathrm{x}^{\prime}\right|}=\mathrm{k}_{0} \frac{\mathbf{x}^{\prime}}{\mathrm{r}}
$$

Using equation 5.6 in 5.4 and ignoring the small changes in the denominator of (5.4) one has

$$
\frac{E_{f}\left(x^{\prime}\right)}{E_{i}}=\frac{e^{+i k_{0} r}}{r} \int e^{i\left(k_{i}-k_{f}\right) \cdot x} e^{+i\left(k_{0} / 2 r\right)\left(x^{2}-\left(k_{f} \cdot x\right)^{2} / k_{0}^{2}\right)} \tilde{N}(x) d^{3} x
$$

The tilde on $\mathrm{N}$ indicates the scattering amplitude has been included in the density:

$$
\tilde{\mathbf{N}}(\mathbf{x}) \equiv N(x) \hat{\mathbf{e}}_{f}^{*} \overrightarrow{\mathbf{F}}\left(\mathbf{k}_{\mathrm{i}}, \mathbf{k}_{\mathrm{f}}, \omega\right) \hat{\mathbf{e}}_{\mathrm{i}}
$$

The tilde on $\mathbf{k}_{\mathrm{f}}$ has been dropped by assuming that the scattering amplitude varies only slowly with angle.

Specular Scattering in the Kinematic Approximation 


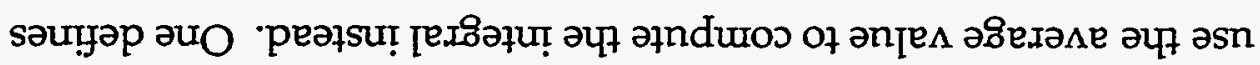

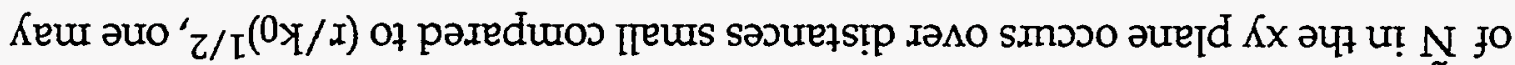

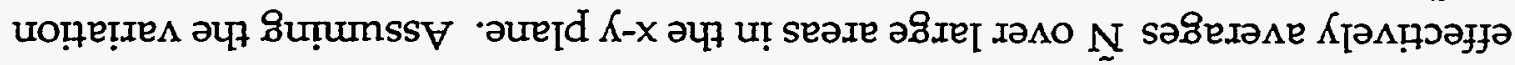

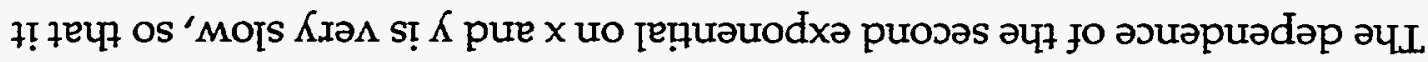

$(Z I \cdot s)$

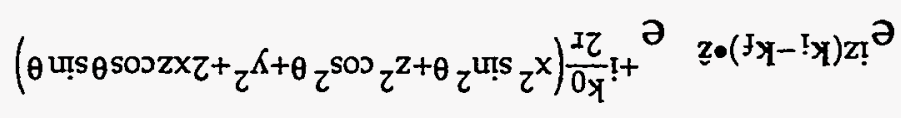

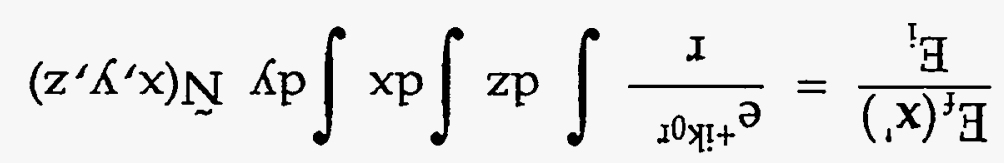

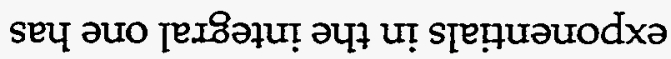

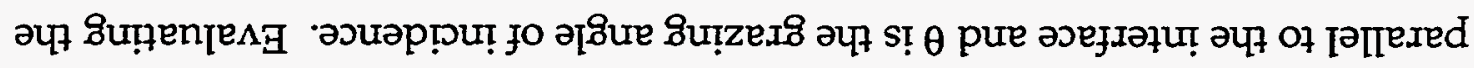

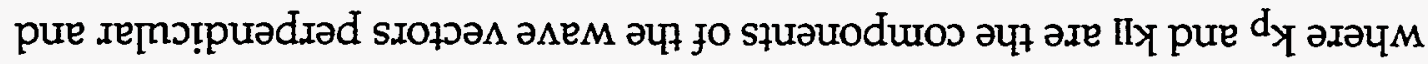

$\left(90 \tau^{\circ} \mathrm{s}\right)$

$$
\begin{aligned}
& \underset{v}{x} \operatorname{sos}^{0} x+z \theta u ! s^{0} x=x^{\pi} x+z^{d} x-={ }^{j} y \\
& x_{\theta} \operatorname{sos}^{0} x_{1}+z z_{\theta} u ! S^{0} x_{-}=x^{I I} X_{1}+z^{d} x_{1}={ }^{!} Y
\end{aligned}
$$

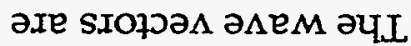

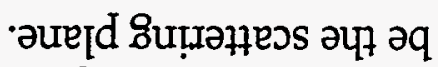

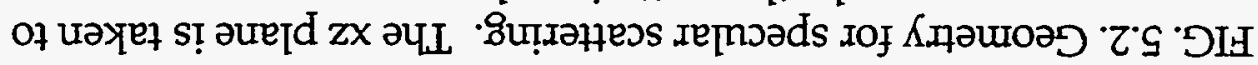

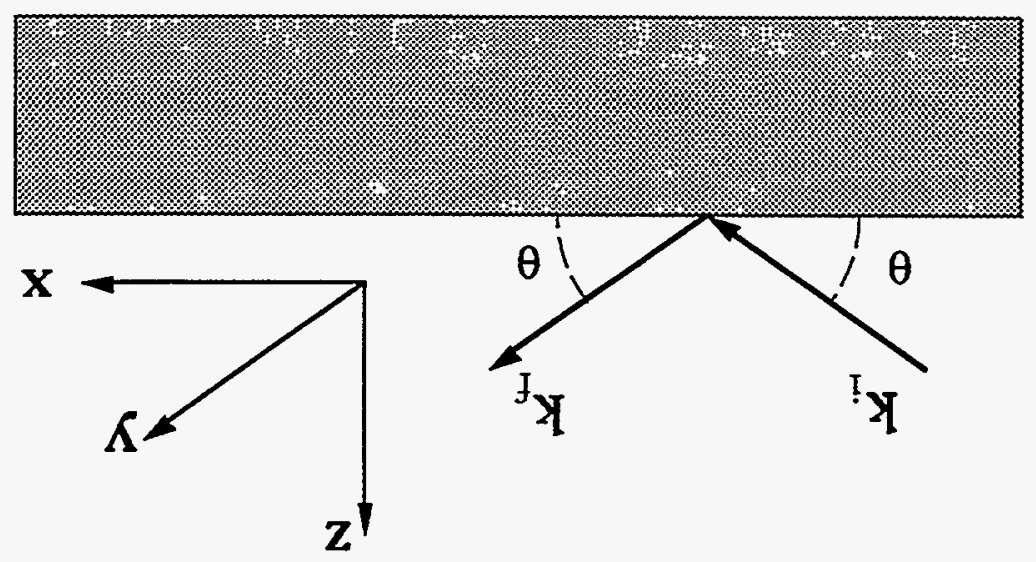

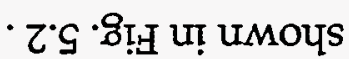

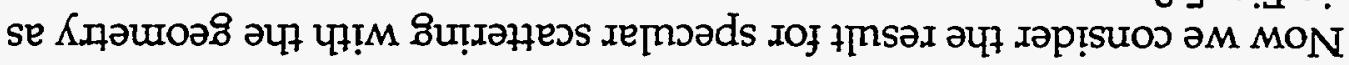




$$
\overline{\tilde{N}}(z) \equiv \frac{1}{L_{x} L_{y}} \int_{-L_{x} / 2}^{L_{x} / 2} d x \int_{-L_{y} / 2}^{L_{y} / 2} d y \tilde{N}(x, y, z)
$$

where $\mathrm{L}_{\mathrm{x}}$ and $\mathrm{L}_{\mathrm{y}}$ are both assumed large compared to any characteristic dimensions of the non-uniformity of the interface (one also needs $\mathrm{L}_{\mathrm{x}}<\left(\mathrm{r} / \mathrm{k}_{0}\right)^{1 / 2} / \sin \theta$ and $\left.\mathrm{L}_{\mathrm{y}}<\left(\mathrm{r} / \mathrm{k}_{0}\right)^{1 / 2}\right)$. Assuming the transverse sample size is also large compared to $\left(r / k_{0}\right)^{1 / 2}$ the limits on the $x$ and $y$ integrals may be taken to infinity. Noting ([Gradshteyn and Ryzhik, 1980, 2], 3.691.1, p. 395)

$$
\int_{-\infty}^{+\infty} d x e^{-i a x^{2}}=\left(\frac{\pi}{2 a}\right)^{1 / 2}(1-i)
$$

one can then evaluate the $x$ and $y$ integrals to give

$$
R \equiv \frac{E_{f}}{E_{i}}=\frac{-2 \pi i}{\left|k_{0} \sin \theta\right|} \int d z e^{-2 i k_{0} z \sin \theta} \overline{\tilde{N}}(z)
$$

where $R$ is just the reflectivity of the interface (and the phase factor has been dropped - the reflectivity is the field ratio extrapolated back to the surface of the sample). Thus the specular reflectivity is just seen to be proportional to the Fourier component of average charge distribution normal to the surface. In the event of more than one type of scatterer, one must, of course, perform a sum over the types of scatterers, with appropriate weighting for their charge distributions and scattering amplitudes.

\section{Kinematic Scattering by a Plane of Scatterers}

Equation (5.15) has the form of a integral over the thickness , $z$, of the sample of the scattering by planes of infinite lateral extent. One identifies the exponential in (5.15) term as being a phase factor for the scattering of the plane at $z$ relative to that at $\mathrm{z}=0$ The reflectivity of a single plane with a scatterer surface density of $\bar{N}(z) d z$ is seen to be (where we have explicitly written out the scattering amplitude for a single type of scatterer) 


$$
R_{\text {plane }}(z)=\frac{-2 \pi i}{\left|k_{0} \sin \theta\right|} \hat{\mathbf{e}}_{f}^{*} \overrightarrow{\mathbf{F}}\left(\mathbf{k}_{\mathrm{i}}, \mathbf{k}_{\mathrm{f}}, \omega\right) \hat{\mathbf{e}}_{\mathrm{i}} \overline{\mathrm{N}}(\mathrm{z}) \mathrm{dz}
$$

This is just the usual result used in x-ray scattering ([Compton and Allison, 1935, 3], pp. 369-371) as a starting point for the dynamical Darwin-Prins theory for symmetric Bragg reflections. It is frequently derived using a Fresnel zone construction ([James, 1962 (Reprinted 1982), 4], p.35, [Henke, et al., 1992, 5]). In fact this expression may also be used as a starting point for deriving the dynamical response of materials at grazing incidence[Smirnov, 1977, 6]. which, for the case of isotropic materials, gives the same results as the treatment based on Maxwell's equations given in the previous chapter. It is worth noting that the Fresnel zones in such descriptions are elliptical with major and minor axes (in the $x$ and $y$ directions, respectively, or parallel and perpendicular to the scattering plane) given by

$$
\begin{aligned}
& a_{n}=\sqrt{n \lambda r} / \sin \theta \\
& b_{n}=\sqrt{n \lambda r}
\end{aligned}
$$

where $\mathrm{n}$ is the index on the zone number. The reflectivity from a plane is proportional to half the area of the first Fresnel zone $\left(\pi a_{1} b_{1} / 2\right)$ (proportionality constant $2 / \pi$ ) and gives the result (5.16) when appropriate phase factors are added. The analogy with the averaging over $L_{x}$ and $L_{y}$ is clear (e.g. equation 5.14): the average requires that any roughness have length scales less than the size of the first Fresnel zone. This is quite large $\left(b_{1}=10 \mu \mathrm{m}\right.$ for $\left.\mathrm{r}=1 \mathrm{~m}, \lambda=1 \AA\right)$ and the transverse scale for roughness is typically small, $<1 \mu \mathrm{m}$ (see e.g. [Sinha, et al., 1988, 7] [Weber and Lengeler, 1992, 8]), so the average performed in (5.14) is reasonable.

\section{Kinematic Scattering by an Ideal Interface}

Now we evaluate (5.15) for the case of an ideal flat interface at $\mathrm{z}=0$. Noting that we are only interested in the scattering due to the presence of the interface, the form for the scattering amplitude density $\tilde{N}$ is just 


$$
\overline{\tilde{N}}(z)=\left\{\begin{array}{ll}
0 & z>0 \\
\hat{\mathbf{e}}_{\mathrm{f}}^{*} \overrightarrow{\mathrm{F}}_{2}\left(\mathbf{k}_{\mathrm{i}}, \mathbf{k}_{\mathrm{f}}, \omega\right) \hat{\mathbf{e}}_{\mathrm{i}} \mathrm{N}_{2}-\hat{\mathbf{e}}_{\mathrm{f}}^{*} \overrightarrow{\mathrm{F}}_{1}\left(\mathbf{k}_{\mathrm{i}}, \mathbf{k}_{\mathrm{f}}, \omega\right) \hat{\mathbf{e}}_{\mathrm{i}} \mathrm{N}_{1} & z<0
\end{array}\right\}
$$

where $\mathrm{N}_{1}$ and $\mathrm{N}_{2}$ are the scatterer number densities above and below the interface, respectively. Evaluation of the integral in (5.16) (assuming a small imaginary component to $k_{p}$ so that the wave will be damped going into the sample) gives

$$
R\left(\theta, \hat{\mathbf{e}}_{i}, \hat{\mathbf{e}}_{f}\right)=\frac{-\pi\left(\hat{\mathbf{e}}_{\mathrm{f}}^{*} \overrightarrow{\mathbf{F}}_{2}\left(\mathbf{k}_{\mathrm{i}}, \mathbf{k}_{\mathrm{f}}, \omega\right) \hat{\mathbf{e}}_{\mathrm{i}} \mathrm{N}_{2}-\hat{\mathbf{e}}_{\mathrm{f}}^{*} \overrightarrow{\mathrm{F}}_{1}\left(\mathbf{k}_{\mathrm{i}}, \mathbf{k}_{\mathrm{f}}, \omega\right) \hat{\mathbf{e}}_{\mathrm{i}} \mathrm{N}_{1}\right)}{\mathrm{k}^{2} \sin ^{2} \theta}
$$

For electronic scattering one has

$$
\hat{\mathbf{e}}_{f}^{*} \overrightarrow{\mathbf{F}}\left(\mathbf{k}_{\mathrm{i}}, \mathbf{k}_{\mathrm{f}}, \omega\right) \hat{\mathbf{e}}_{\mathrm{i}} \rightarrow-\hat{\mathbf{e}}_{\mathrm{f}}^{*} \bullet \hat{\mathbf{e}}_{\mathrm{i}} \mathrm{r}_{\mathrm{e}} \mathrm{f}(2 \theta, \omega)
$$

Considering scattering of sigma polarized light into sigma polarized light, the polarization factor becomes unity so the electronic reflectivity becomes

$$
R(\theta, \hat{\sigma}, \hat{\sigma})=\frac{\pi r_{e}}{k_{0}^{2} \sin ^{2} \theta}\left(N_{2} f_{2}(2 \theta, \omega)-N_{1} f_{1}(2 \theta, \omega)\right)
$$

We may compare directly with the result from the optical theory (4.42a) in the weak scattering limit $\left(\theta \gg \theta_{c}\right)$. Expanding (4.53b) to lowest order in $\delta / \sin ^{2} \theta$ gives

$$
R_{\text {opt }}(\theta, \hat{\sigma}, \hat{\sigma})=\frac{\delta_{2}-\delta_{1}}{2 \sin ^{2} \theta}=\frac{\pi r_{e}}{k_{0}{ }^{2} \sin ^{2} \theta}\left(N_{2} f_{2}(0, \omega)-N_{1} f_{1}(0, \omega)\right)
$$

The only difference between the two forms is that the angular dependence of the scattering amplitude does not appear in the optical theory. This thesis is only concerned with scattering at small angles, where $f(2 \theta) \sim f(0)$, so this is not of great concern here. In general, however, this is a result of the assumption of homogeneity in the optical theory (see equations (4.14) and (4.15)). This means that one must consider the scatterers to be uniformly spread out and hence there can be no effects due to their internal structure, which is precisely what $f(2 \theta>0)$ describes. 


\section{Kinematic Scattering From a Real (Non-Ideal) Interface}

To discuss the scattering from a non-ideal or real interface it is necessary to specify the form of the roughness or grading of the interface. It is convenient to do this by introducing a function $\mathrm{w}$, for the normalized derivative of the densities with position. $w$ is defined by

$$
\begin{aligned}
& N_{1}(z)=N_{1} \int_{-\infty}^{z} w(z) d z \\
& N_{2}(z)=N_{2} \int_{z}^{+\infty} w(z) d z
\end{aligned}
$$

where $N_{1}$ is the number density of scatters far above the interface and $N_{2}$ is that far below the interface ( $w$ is normalized to unity). Inverting these one has

$$
w(z)=\frac{1}{N_{1}} \frac{d}{d z} N_{1}(z)=-\frac{1}{N_{2}} \frac{d}{d z} N_{2}(z)
$$

The form of $\tilde{N}$ is then taken to be (where, again, we have subtracted off $\mathrm{N}_{1}$ )

$$
\begin{aligned}
\overline{\tilde{N}}(z) & =\hat{\mathbf{e}}_{\mathrm{f}}^{*} \overrightarrow{\mathrm{F}}_{2}\left(\mathbf{k}_{\mathrm{i}}, \mathbf{k}_{\mathrm{f}}, \omega\right) \hat{\mathbf{e}}_{\mathrm{i}} \overline{\mathrm{N}}_{2}(z) \\
& +\hat{\mathbf{e}}_{\mathrm{f}}^{*} \overrightarrow{\mathrm{F}}_{1}\left(\mathbf{k}_{\mathrm{i}}, \mathbf{k}_{\mathrm{f}}, \omega\right) \hat{\mathbf{e}}_{\mathrm{i}}\left(\overline{\mathrm{N}}_{1}(\mathrm{z})-\mathrm{N}_{1}\right)
\end{aligned}
$$

Inspection shows that straightforward substitution of this into (5.15) leads to divergent integrals. The divergent terms will cancel out if appropriate care is taken. Doing this shows the result to be equivalent to making the following substitutions, suggested by Fourier analysis:

$$
\begin{aligned}
& \int_{-\infty}^{+\infty}\left(\bar{N}_{1}(z)-N_{1}\right) e^{i q z} d z \rightarrow \frac{i}{q} \int_{-\infty}^{+\infty} \frac{d \bar{N}_{1}(z)}{d z} e^{i q z} d z \\
& \int_{-\infty}^{+\infty} \bar{N}_{2}(z) e^{i q z} d z \rightarrow \frac{i}{q} \int_{-\infty}^{+\infty} \frac{d \bar{N}_{2}(z)}{d z} e^{i q z} d z
\end{aligned}
$$

The reflectivity becomes 


$$
\begin{aligned}
& \mathrm{R}\left(\theta, \hat{\mathbf{e}}_{\mathrm{i}}, \hat{\mathbf{e}}_{\mathrm{f}}\right)=\frac{-\pi}{\mathrm{k}_{0}^{2} \sin ^{2} \theta} w(2 \mathrm{k} \sin \theta) \\
& \quad \times\left\{\mathrm{N}_{2} \hat{\mathbf{e}}_{\mathrm{f}}^{*} \overrightarrow{\mathrm{F}}_{2}\left(\mathbf{k}_{\mathrm{i}}, \mathbf{k}_{\mathrm{f}}, \omega\right) \hat{\mathbf{e}}_{\mathrm{i}}-\mathrm{N}_{1} \hat{\mathbf{e}}_{\mathrm{f}}^{*} \overline{\mathrm{F}}_{1}\left(\mathbf{k}_{\mathrm{i}}, \mathbf{k}_{\mathrm{f}}, \omega\right) \hat{\mathbf{e}}_{\mathrm{i}}\right\}
\end{aligned}
$$

where $w(q)$ is just the Fourier transform of $w$, given by

$$
w(q) \equiv \int_{-\infty}^{\infty} w(z) e^{i q z} d z
$$

Comparing (5.27) with (5.21) the form of the correction for a non-ideal interface is just seen to be

$$
\mathbf{R}_{\text {real }}\left(\theta, \hat{\mathbf{e}}_{i}, \hat{\mathbf{e}}_{\mathrm{f}}\right)=\mathbf{R}_{\text {ideal }}\left(\theta, \hat{\mathbf{e}}_{i}, \hat{\mathbf{e}}_{\mathrm{f}}\right) w(q)
$$

which agrees with the result given by other authors (see, e.g. [Stearns, 1988, 9])

In particular, it is often assumed that the (average) number densities have an error function dependence on $\mathrm{z}$. Then $\mathrm{w}(\mathrm{z})$ is Gaussian:

$$
w(z)=\frac{1}{\sqrt{2 \pi} \sigma} e^{-(z)^{2} / 2 \sigma^{2}}
$$

The width of the transition region is just given by $\sigma$. The Fourier transform of a Gaussian is just a Gaussian

$$
w(\mathrm{q})=\frac{1}{\sqrt{2 \pi} \sigma} \int_{-\infty}^{+\infty} \mathrm{e}^{-\left(z^{\prime}\right)^{2} / 2 \sigma^{2}} \mathrm{e}^{\mathrm{iq} z} \mathrm{dz}=\mathrm{e}^{-\mathrm{q}^{2} \sigma^{2} / 2}
$$

So that one has

$$
R_{\text {real }}=R_{\text {ideal }} e^{-2 \mathrm{k}_{\mathrm{p}}^{2} \sigma^{2}} \quad\left(\begin{array}{l}
\text { Error Function } \\
\text { Interface Profile }
\end{array}\right)
$$


Figure (5.2) shows the effect that $5 \AA$ roughness (i.e. $\sigma=5 \AA$ ) has on the reflectivity from bulk density iron as a function of angle. One should note that there are substantial differences at grazing angles above $10 \mathrm{mrad}$.

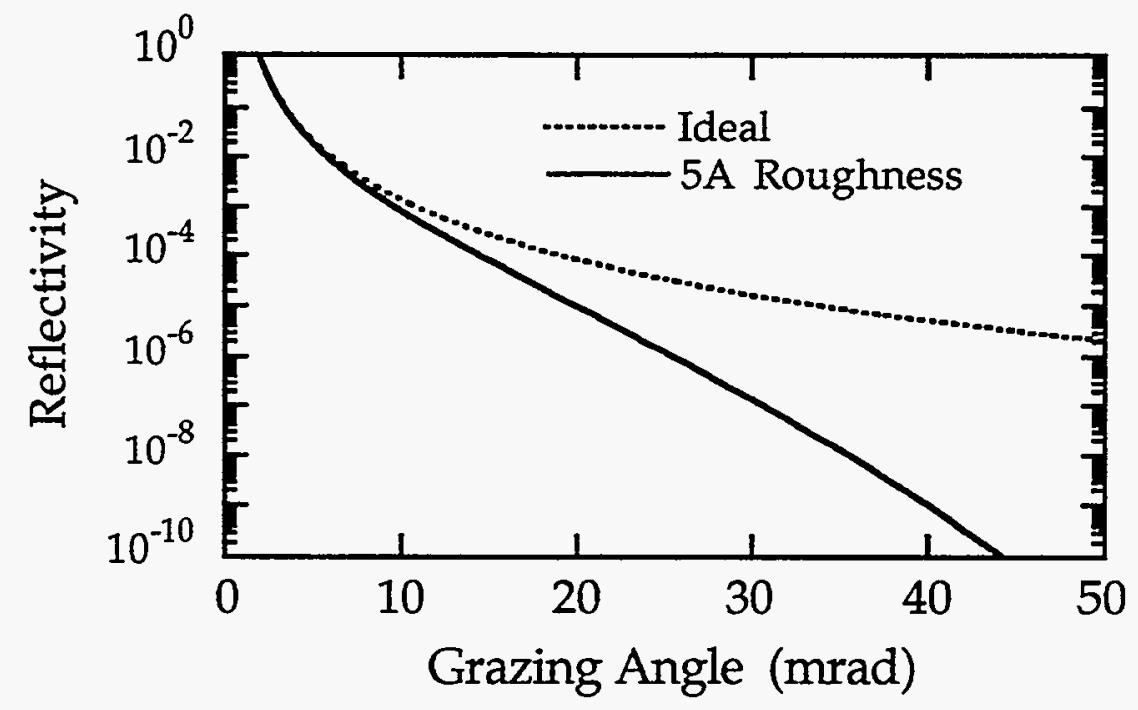

Figure 5.2. Effect of roughness on the reflectivity of bulk density iron.

Failure of the Kinematic Result at Grazing Incidence.

At small grazing angles of incidence $\left(\theta \sim \theta_{c}\right)$ the reflected wave becomes large and the assumptions that allow a kinematic treatment fail. This is immediately evident by noting that expressions (5.21) and (5.27) both blow up at small angles (also see fig. 5.2). However, one notes that if the ideal reflectivity in (5.29) is just taken to be the reflectivity from the optical theory in the previous chapter, the expression (5.29) remains well defined at small angles. This is plotted in Fig. 5.3 and, indeed, the blow up of the pure kinematic response at small angles is prevented.

However, it is not obvious that, in the immediate vicinity of the critical angle the correction in (5.32) is right. In particular, the perpendicular components of the wave vectors above and below the interface can be very 
different, due to refraction of the wave, so the dependence of the roughness correction on only one of these vectors (or the free space vector) is annoying

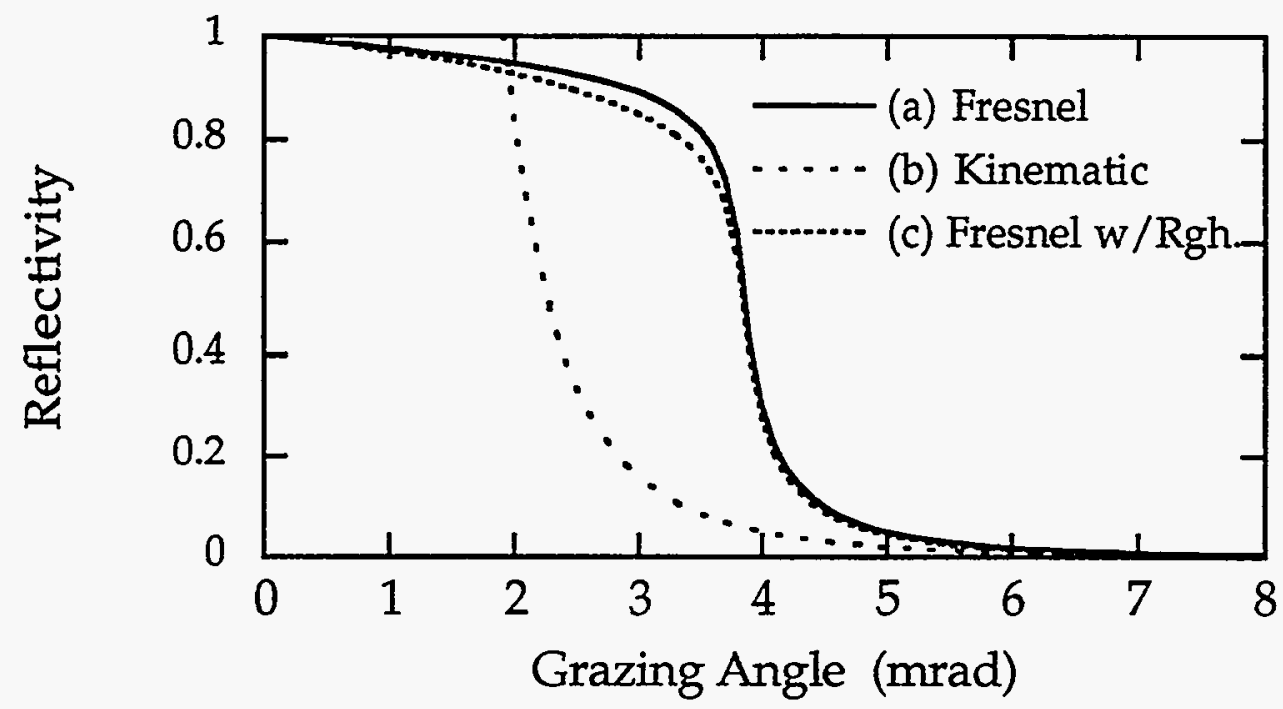

Figure 5.3. Fresnel reflectivity modified for roughness $(\sigma=5 \AA)$. (a) shows the ideal Fresnel reflectivity for the interface without roughness, (b) the kinematic calculation (no roughness) and (c) the Fresnel reflectivity with the roughness correction of equation (5.32)

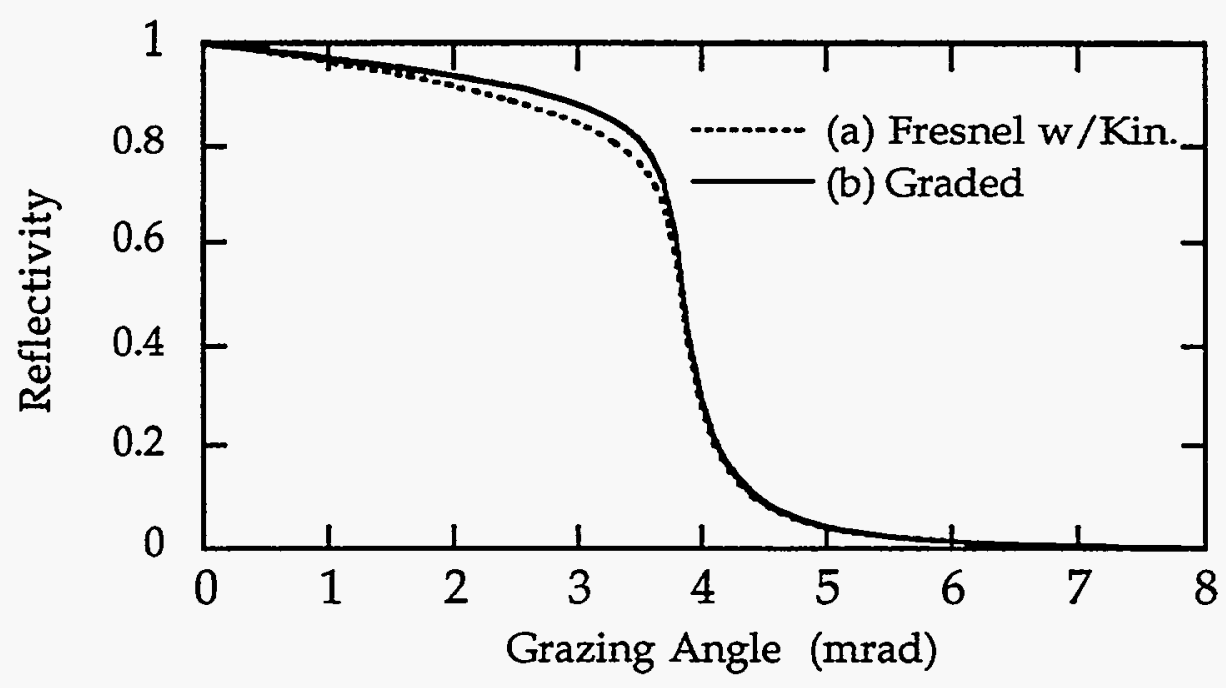


Figure 5.4. (a) Fresnel reflectivity with the kinematic roughness correction $(\sigma=5 \AA)$ as compared with (b) the full calculation for a graded interface.

and probably not correct. An estimate of the validity of this modified version of (5.32) in this region can be obtained by comparing this calculation to the exact calculation of a graded interface: based on the discussion above, the correct treatment of roughness should give identical results to that of a graded interface.

Figure 5.4 shows that the modified Fresnel response is not such a good approximation to the correct solution (defined as the graded interface calculation) in the vicinity of the critical angle and it actually gets worse quickly as the roughness, $\sigma$, increases. However, near the critical angle, the perpendicular components of the wave vectors above and below the interface are very different due to refraction. Thus, for the simple case of isotropic materials there is some temptation to simply replace one of the wave vectors in (5.32) by the interior wave vector. Namely, we try

$$
R_{N C}=R_{\text {Fresnel }} e^{-2 k_{p} k_{p}^{\prime} \sigma^{2}}
$$

where the prime indicates the wave vector below the surface. This turns out to be a very good approximation, as has been shown in a couple of papers, [Nevot and Croce, 1980, 10, Sinha, et al., 1988, 7]. The subscript NC indicates "Nevot and Croce", who first presented this result. If plotted in figure (5.4), it would lie exactly on top of the graded interface calculation.

\section{Why Bother With Roughness Corrections?}

It is worth a moment to discuss the usefulness of roughness corrections. Earlier in the chapter we showed that, for any case we care about, the rough interface can be reduced to a graded interface which can then be calculated using the formalism of the previous chapter. On the one hand, however, it is nice to have an analytic form for how non-ideal interfaces affect the reflectivity. On the other, it is very convenient calculationally, simply because the number of layers 
required to model a rough interface properly can be quite large and thus a significant hindrance to calculations. A simple correction is very desirable.

Before investigating (5:33) in more detail, however, it is worth mentioning that another approach to the problem is to "simply" solve Maxwell's equations in a medium with a known (1-dimensional) profile for the index of refraction. For the simplest case of sigma radiation incident on an isotropic medium (e.g. electronic scattering), the relevant equation is the scalar wave equation, with a depth dependent wave vector:

$$
\begin{aligned}
& \frac{d^{2}}{d z^{2}} E(z)+k_{p}{ }^{2}(z) E(z)=0 \\
& k_{p}{ }^{2}(z)=k_{0}{ }^{2}\left(\sin ^{2} \theta-2 \delta(z)\right)
\end{aligned}
$$

where $E$ is the sigma component of the electric field and $\delta(z)$ is the local decrement of the index of refraction from one. In the case of a linear variation in $\delta(z)$, the result may be expressed in terms of Airy functions [Smirnov, 1977, 6], but for an error function, or a more general distribution, this author has not been able to find a convenient solution. In addition, of course, when a more complex system is investigated (i.e. a non-isotropic nuclear system, the equation becomes somewhat more complex). Thus we are left with approximation methods, if we would like an analytic form for a roughness correction.

\section{The Distorted Wave Born Approximation}

The result, (5.33), that provides a reasonable roughness correction for electronic scattering was originally given by Nevot and Croce ([Nevot and Croce, $1980,10]$,[Nevot, et al., 1988, 11]) who found it to be the lowest order correction from averaging over various rough surface configurations. However, more recently, Sinha et al, [Sinha, et al., 1988, 7], have presented a very nice derivation using a distorted wave Born approximation (DWBA) (see, e.g. [Schiff, 1968, 12], pp. 324-328). In concept, the work of Sinha et al, is actually very similar to the 
distorted wave approximation discussed earlier by Vineyard, [Vineyard, 1982, 13 , though Vineyard did not explicitly consider roughness problems.

Appendix B contains a detailed discussion of the DWBA result and the generalization to anisotropic media. At the level of the main text here, we only quote the relevant (often used) result for isotropic (electronic) scattering at grazing incidence [Nevot and Croce, 1980, 10], [Sinha, et al., 1988, 7], [Weber and Lengeler, 1992, 8]

$$
\begin{aligned}
& R_{N C}=R_{\text {Fresnel }} e^{-2 k_{p} \dot{k}_{p}^{\prime} \sigma^{2}} \\
& T_{N C}=T_{\text {Fresnel }} e^{+\left(k_{p}-k_{p}^{\prime}\right)^{2} \sigma^{2} / 2}
\end{aligned}
$$

The relevant question, of course, is whether or not these may be applied to nuclear scattering as well. In general they do quite nicely for electronic scattering. Ignoring polarization effects (which are discussed in appendix B) the only pertinent difference between electronic scattering and nuclear scattering is that for electronic scattering one always has $\operatorname{Im}\{\delta\}<<\operatorname{Re}\{\delta\}$, while for nuclear scattering one may have $\operatorname{Im}\{\delta\}>\sim \operatorname{Re}\{\delta$. We must examine the validity (5.36) in the case there is large absorption.

\section{Roughness Correction in a Highly Absorbing Material}

We can estimate the effectiveness of the roughness correction for the nuclear response by just looking at the reflectivity as a function of angle for unusual values of delta. In particular, figure 4.3 , shows a plot of delta near a nuclear resonance in enriched ${ }^{57} \mathrm{Fe}$. In fact, since the result is appropriate for unsplit iron, it turns out to have somewhat larger values of delta than any of the experimental cases actually described here, and is therefore a good "worse case" limit. Taking $\delta=5 \times 10^{-}-i_{i}$, a worst case on resonance limit, figure 5.5 shows the effects of the roughness and the error in the Nevot-Croce calculation. 


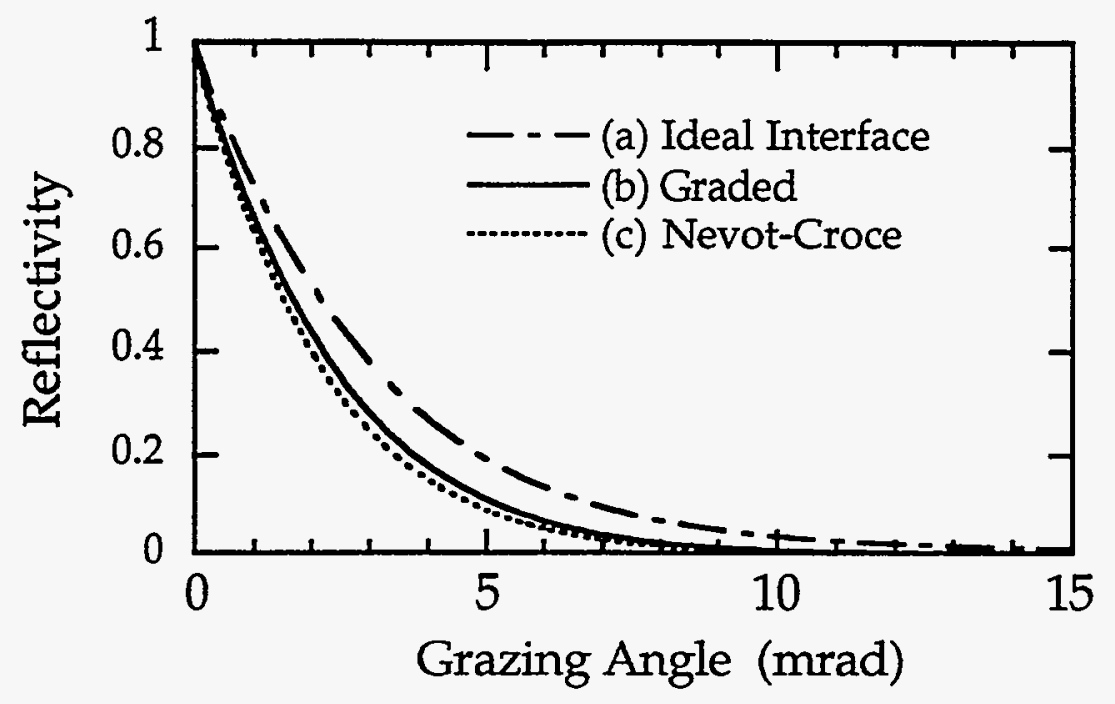

Figure 5.5. Effect of $\sigma=10 \AA$ roughness on the reflectivity of a sample in the vicinity of a resonant transition. Calculation for $\delta=5 \times 10^{-5} \mathrm{i}$ and (a) an ideal (smooth) interface, (b) a graded interface $(\sigma=10 \AA)$ and (c) using the Nevot-Croce correction for a rough interface $(\sigma=10 \AA)$.

Two things are immediately apparent from figure 5.5. First, unlike normal electronic materials where the effect of (small amounts of) roughness largely disappear at grazing angles, the effects of roughness on the reflectivity in the vicinity of a nuclear transition can be significant. Secondly, in this case, the Nevot-Croce approximate result for a rough interface is poor, deviating from the exact result for a graded interface by a large amounts. Numerical investigation of the effect of roughness and the validity of the Nevot-Croce approximation suggest that in general, when delta and sigma are large in magnitude, the effect of roughness, even at grazing incidence, is significant. However, if only the real part of delta is large, then these effects are well approximated by the NevotCroce approximation. This is evident in figure 5.6, which is the reflectivity calculated for delta real and large (with the same $\sigma=10 \AA$ used in fig 5.5): the Nevot-Croce approximation overlays the calculation for the graded interface very nicely, with differences of only a few percent. 


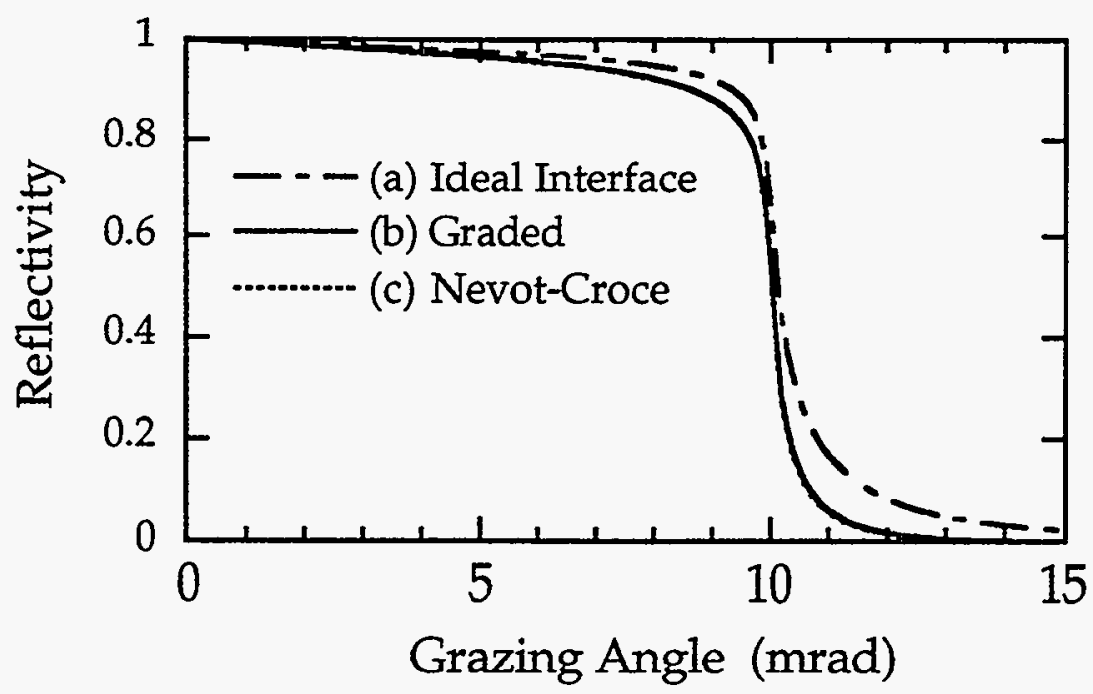

Figure 5.6. Effect of $\sigma=10 \AA$ roughness on the reflectivity of a sample in the vicinity of a resonant transition. Calculation for $\delta=5 \times 10^{-5}+\mathrm{i} \times 10^{-6}$ and (a) an ideal (smooth) interface, (b) a graded interface $(\sigma=10 \AA)$ and (c) using the Nevot-Croce correction for a rough interface $(\sigma=10 \AA)$.

One expects that the reason why the Nevot-Croce form fails for large imaginary delta is the severity of the absorption. The $1 / \mathrm{e}$ penetration length (intensity) for $\mathrm{x}$-rays into a material is just $\lambda / 4 \pi \operatorname{Im}\{\delta\}$, which gives an absorption length of $1400 \AA$ for $\operatorname{Im}\{\delta\}=5 \times 10^{-5}$ and $14.4 \mathrm{keV}$ radiation $(\lambda=0.86 \AA)$. Thus, photons on resonance incident at an angle of $5 \mathrm{mrad}$, have a 1/e attenuation length of $7 \AA$ normal to the surface. Thus, considering the derivation in Appendix C, through the DWBA which is essentially a first order perturbation calculation, it is not at all surprising that the Nevot-Croce result fails. Conversely, now, one should probably ask why it succeeds at all when the real part is so large. However, fortunately, it seems to work nicely. This may be related to the fact that the imaginary part of delta, the absorption depletes the beam intensity, independent of the location, while the real part results in a phase change and thus the DWBA corrections to the scattering from above the interface and below the interface may partially cancel. 
Finally, the question is whether or not it is reasonable to use the NevotCroce correction in the analysis of data. The answer really depends on the sample being considered: on an immediate level, the Nevot-Croce result seems valid whenever the real part of delta is much larger than the imaginary part and this will always be the case except in the immediate vicinity of a resonant transition. The above were basically worst case estimates: one really needs to evaluate the response for parameters appropriate to the sample being considered. In particular, the iron layer sample discussed in the next section, the characteristic roughness was about $4 \AA$ and the nuclear properties such that the imaginary part of delta does not exceed about $2 \times 10^{-5}$. In this case, though roughness can change the reflectivity by as much as $30 \%$ at angles less than 10 mrad, the Nevot-Croce correction agrees with the graded interface calculation to $1 \%$ or better. Thus, while roughness should be included, it will only be included though the Nevot-Croce correction, or the full DWBA described in appendix B.

\section{References for Chapter 5}

1 L.A. Smirnov, T.D. Sotnikova, B.S. Anokhin, and B.Z. Taibin, Total external reflection of $x$-rays from rough surfaces. Opt. Spectrosk. 46 (1979) 593.

2 I.S. Gradshteyn and I.M. Ryzhik, Table of Integrals, Series and Products. New York: Academic Press, Inc. (1980).

3 A.H. Compton and S.K. Allison, X-Rays in Theory and Experiment. Second Edition ed. New York: D. Van Nostrand Company, Inc. (1935).

4 R.W. James, The Optical Principles of the Diffraction of X-rays. Woodbridge, Connecticut: Ox Bow Press (1962 (Reprinted 1982)).

5 B.L. Henke, E.M. Gullikson, and J.C. Davis, X-ray interactions: photoabsorption, scattering, transmission and reflection. Atomic Data and Nuclear Data Tables 54 (1992) 181.

6 L.A. Smirnov, Opt. Spectrosk. 43 (1977) 567.

7 S.K. Sinha, E.B. Sirota, and S. Garoff, X-ray and neutron scattering from rough surfaces. Phys. Rev. B 38 (1988) 2297. 
8 W. Weber and B. Lengeler, Diffuse Scattering of hard $x$-rays from rough surfaces. Phys. Rev. B 46 (1992) 7953.

9 D.G. Stearns, The scattering of $x$ rays from non-ideal multilayer structures. J. Appl. Phys. 65 (1988) 491.

10 L. Nevot and P. Croce, Caracterisation des surfaces par reflexion rasante de rayons $X$. Application a l'etude du polissage de quelques verres silicates. Revue Physique. Appliquee 15 (1980) 761.

11 L. Nevot, B. Pardo, and J. Corno, Characterization of X-UV multilayers by grazing incidence $x$-ray reflectometry. Revue Phys. 23 (1988) 1675.

12 L.I. Schiff, Quantum Mechanics. New York: McGraw-Hill Book Co. (1968).

13 G.H. Vineyard, Grazing incidence diffraction and the distorted wave approximation for the study of surfaces. Phys. Rev. B 26 (1982) 4146. 


\section{The Specular Response of a Thin Layer of $57 \mathrm{Fe}$}

\section{Introduction}

This chapter contains the analysis of total external reflection measurements from a thin $(240 \AA)$ layer of $57 \mathrm{Fe}$ deposited on a glass substrate. The electronic and nuclear (time domain) reflectivity are discussed in detail. This work is one of the first attempts to apply synchrotron resonant nuclear scattering techniques to investigate a sample where the structure was not precisely known.

This work is essentially the time domain analog to experiments that were carried out in the 60 's by Bernstein and Campbell [Bernstein and Campbell, 1963, 1] using a radioactive Mössbauer source. It is similar (in choice of sample) to more recent work by the Japanese synchrotron Mössbauer collaboration which was presented at the 1992 International Conference on Anomalous Scattering* (ICAS) in Malente, Germany [Kikuta, et al., 1992, 2]. Kikuta, et al., measured the time evolution of the radiation specularly reflected from a "57Fe mirror" ( $40 \%$ enrichment). They presented "simulations" of the data based on the theory in [Hannon, et al., 1985, 3], which reproduced many of the features of the data. However, no detailed analysis was presented, or, to this author's knowledge, has been presented since.

The thin film sample used in these investigations, is also, perforce, similar to the thin film samples used in the grazing incidence anti-reflection (GIAR) film work long pursued by Gerdau's group in Hamburg, Germany[Grote, et al., 1989, 4, Grote, et al., 1991, 5, Röhlsberger, et al., 1992, 6, Röhlsberger, et al., 1993, 7]. However, the emphasis of such work, as discussed in chapter 2 , is to provide a very large nuclear bandwidth $\left(>\sim 100 \Gamma_{0}\right)$ so the time response is very fast and not easily measured. Only two time responses from GIAR films have been presented in the literature[Grote, et al., 1991, 5, Alp, et al., 1993, 8], and while an effect is certainly visible, the data is not of sufficient quality for detailed analysis. There

\footnotetext{
* Initial results from the sample dicussed here were presented at this conference as well.
} 
is little practical overlap between the analysis of the data here and the GIAR work, although, as discussed below, there are certainly some conceptual similarities.

In the field of synchrotron radiation based studies of nuclear scattering, this work is very much unique. Its value, in comparison to other techniques, will be discussed at the end of the chapter.

\section{Sample Preparation and Characterization}

This sample was made by magnetron sputtering at the Applied Physics Institute in Niznhii Novgorod, Russia by N.N. Salashchenko and S.I. Shinkarev. The source was a $95 \%$ enriched ${ }^{57} \mathrm{Fe}$ target and the sputtering was carried out in $6 \mathrm{mTorr}$ of Ar. The substrate was flat optical glass, $30 \mathrm{~mm} \times 60 \mathrm{~mm}$, with an expected surface roughness of about $5 \AA$.

The sample has been investigated by conversion electron Mössbauer spectroscopy (CEMS) and Mössbauer total external reflection measurements [Isaenko, et al., 1994, 9] using a radioactive source at the Russian Research Center "Kurchatov Institute" in Moscow, Russia. At Stanford, the grazing incidence specular X-ray reflectivity was measured, as well as the sputter Auger profile. The time dependence of the specular nuclear scattering was measured at the Cornell High Energy Synchrotron Source (CHESS). The results from each of the measurements will be discussed below.

\section{Electronic Reflectivity}

Figure 6.1 shows the non-resonant reflectivity of the iron layer for 14.4 $\mathrm{keV} \mathrm{x}$-rays measured out to $45 \mathrm{mrad}$. There are 22 small oscillations in a range of about $39 \mathrm{mrad}$, corresponding to an average period of $1.77 \mathrm{mrad}$. These oscillations are due to interference of the waves reflected off the front and back surfaces of the iron layer (the air-iron and iron-glass interfaces, respectively) and were first observed by Kiessig [Kiessig, 1931, 10]. Their period suggests an 
approximate thickness of the layer of 243 angstroms*, while fitting the response with a single layer of iron on glass seems to give good alignment of the interference minima with a thickness of $240 \AA$.

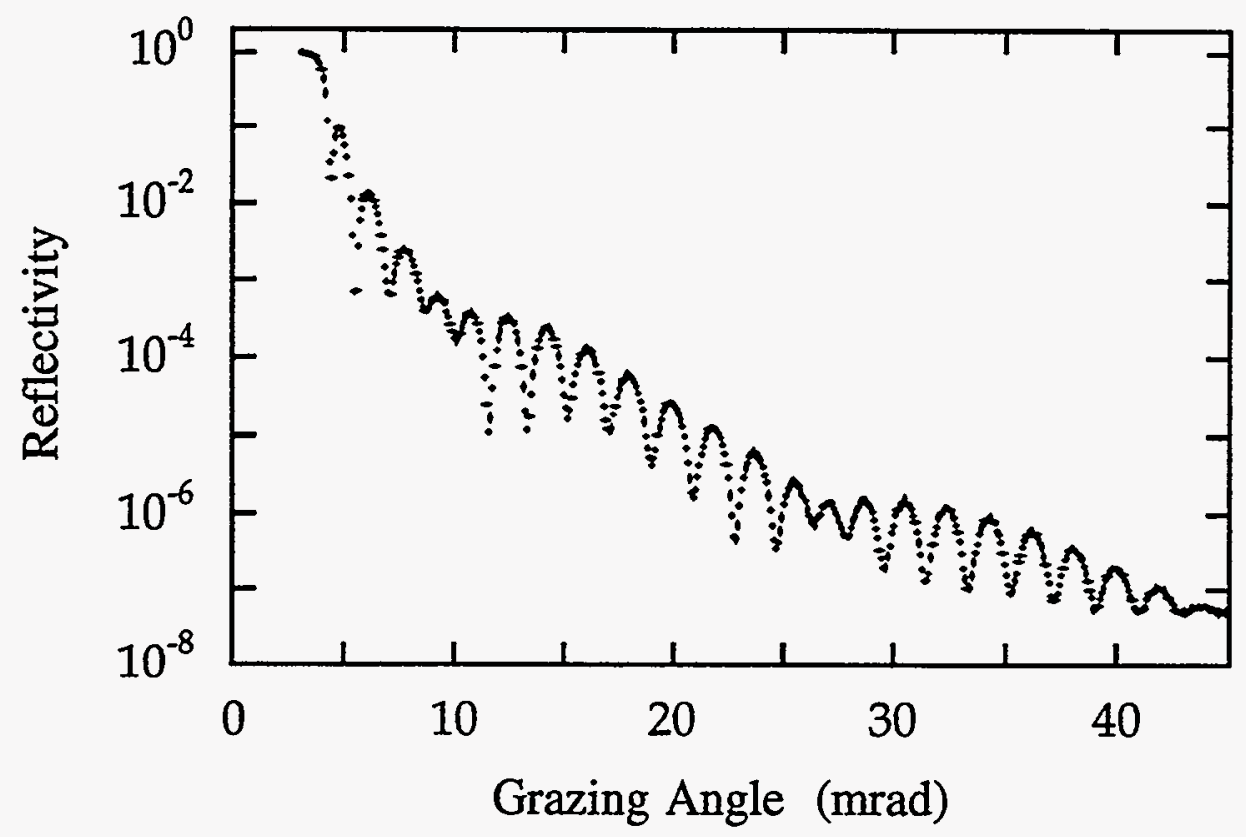

Figure 6.1. X-ray reflectivity of the iron layer at $14.4 \mathrm{keV}$.

However, there is substantial subsidiary structure in the reflectivity curve that can not be accounted for with a simple two layer (iron on glass) model. In particular, one notes modulation of the maxima and modulation of the minima at different frequencies. This suggests a more complex structure. Computer modeling shows that this type of modulation is consistent with a density profile in which the iron is divided into three layers(two layers can not produce modulation of the maxima and minima with different periods). Fitting a 3 layer profile to the data then gives the result shown in figure 6.2. Figure 6.2a shows the measured and calculated reflectivity for the index of refraction profile shown in figure $6.2 \mathrm{~b}$. The fit reproduces the overall structure of the measured curve quite well. Note that the smoothness of interface structure shown in $6.2 \mathrm{~b}$ is from

\footnotetext{
* The interference condition, or Braggs law, for this case is just $\lambda=2 \mathrm{~d} \theta_{\mathrm{p}}$, where $\lambda=0.860 \AA$, the wavelength of light, $d$ is the thickness of the layer and is $\theta_{p}=1.77 \mathrm{mrad}$ is the period of the oscillation. Index of refraction effects are ignored.
} 

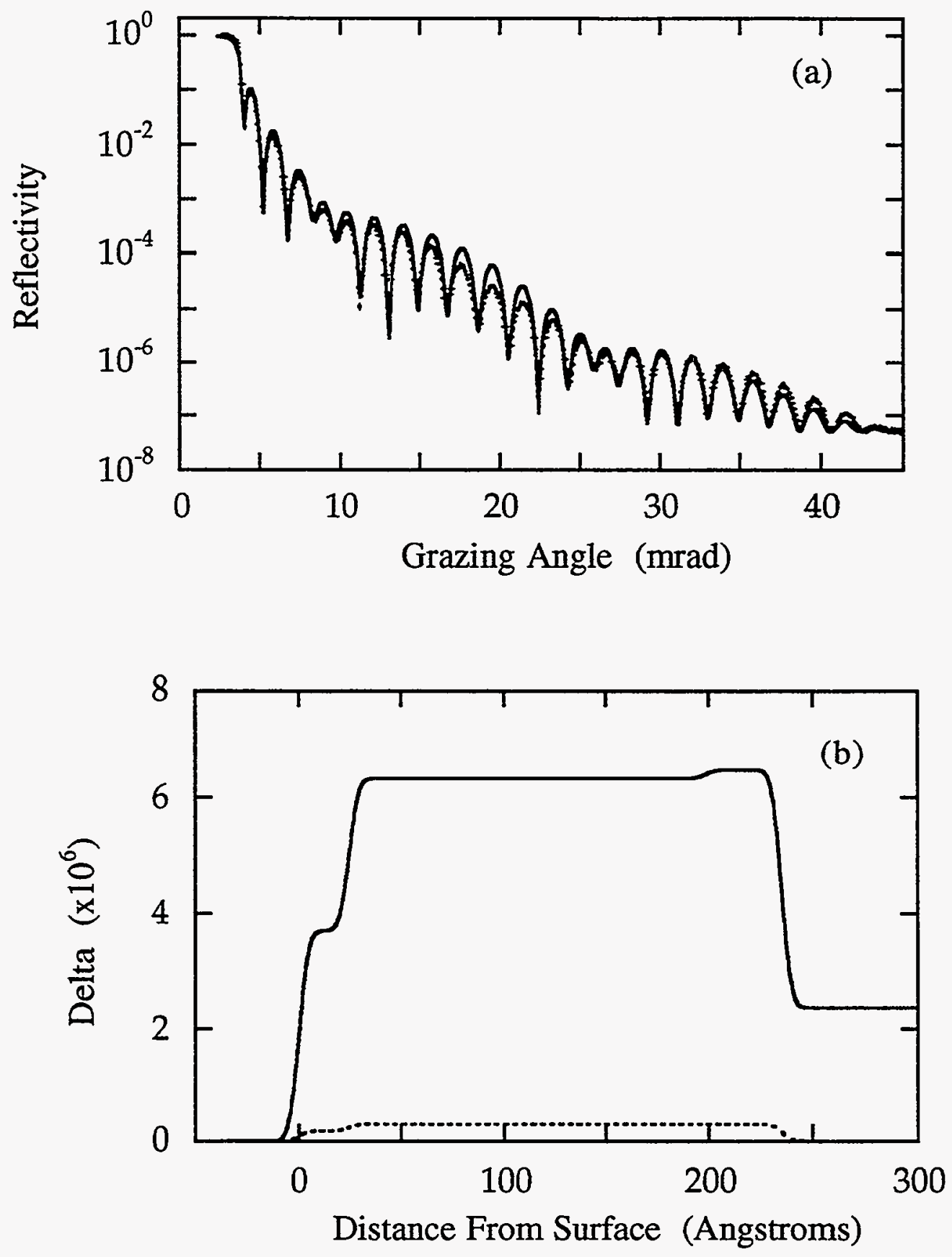

Figure 6.2. Three layer model of the iron layer. (a) Shows the calculated reflectivity (solid line) and the measurement (points) while (b) the index of refraction (real part = solid line, imaginary part $=$ dashed line) used in the calculation. 

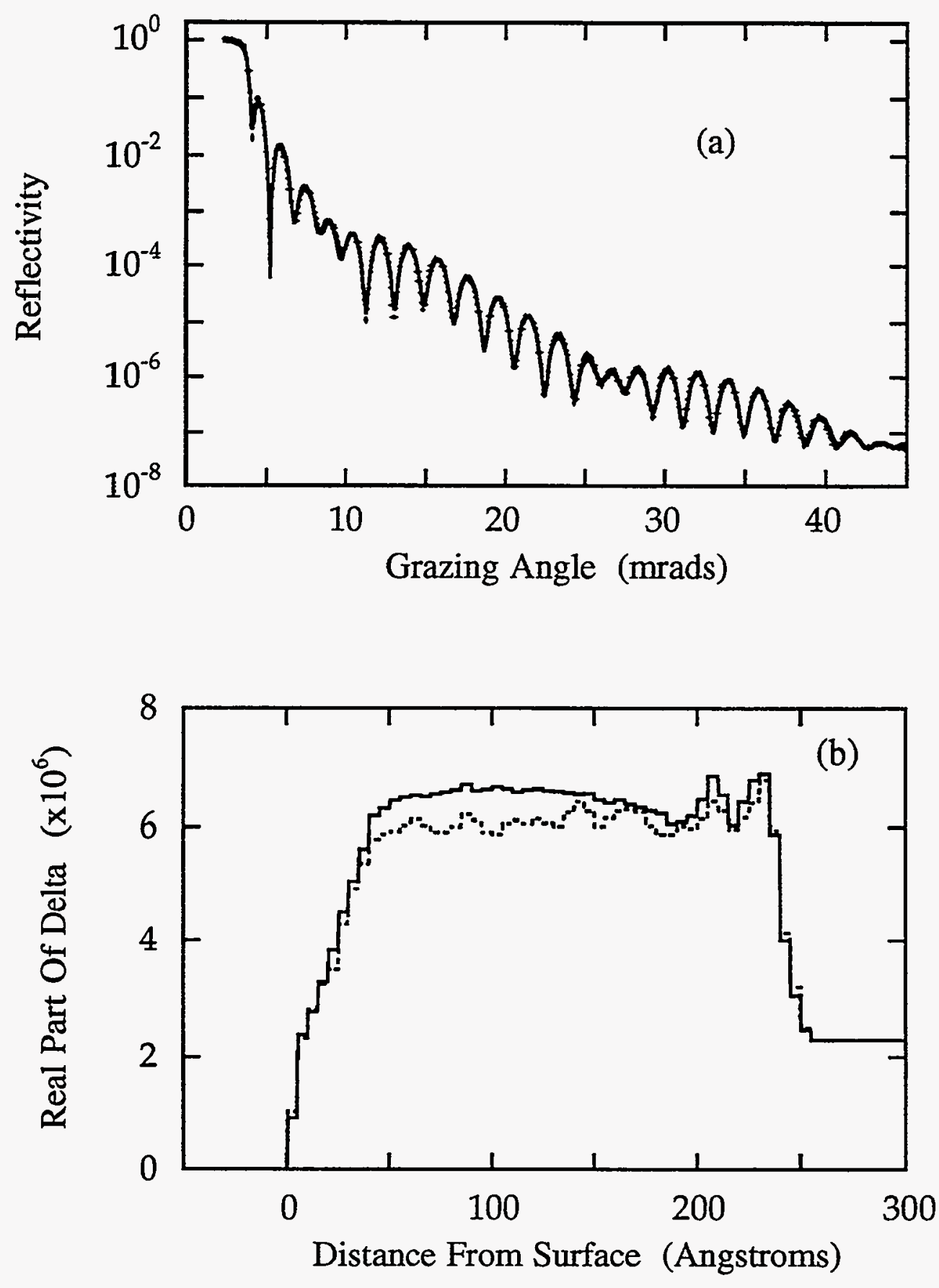

Figure 6.3. Many layer models of the iron layer. (a) Measured and calculated reflectivity profiles. (b) Real part of the index of refraction $(\delta=1-n)$ model used to generate the fit in (a). The dashed line in (b) shows the model actually used in (a) while the solid line shows an alternative model generating an essentially indistinguishable fit. 
assuming rough interfaces ( $\sigma=3.7 \AA$ ) and that while the response at small angles is not sensitive to the roughness, the response at large angles is very sensitive to it.

In principle, it does not seem unreasonable to fit the reflectivity by allowing the density to vary almost continuously as a function of depth. With information out to $\theta_{\max }=45 \mathrm{mrad}$, one would expect resolution to about $10 \AA=$ $\lambda / 2 \theta_{\max }$. Dividing up the layer into $5 \AA$ pieces, a very pretty fit was obtained, as is shown in figure 6.3a. The index of refraction for this model is shown in figure $6.3 \mathrm{~b}$ and is in reasonable agreement with that of the three layer model.

Finally, what is truly important is how well these models describe the actual structure of the layer. One must immediately address questions of the uniqueness: that a fitting program (with substantial human help) came up with one minimum, does not, in this very complicated parameter space guarantee that there are not other, possibly better, minima corresponding to other models of the structure. In fact, figure $6.3 \mathrm{~b}$ shows two models that generate fits to the data that are essentially equivalent. Thus, a unique determination of the electron density profile of the sample is not possible based on the x-ray diffraction data*.

However, one can make some general comments about the structure of the layer. All fits are consistent with what is essentially a three layer model: there is an interface region (about $40 \AA$ ) of reduced electron density at the surface, a region (about $160 \AA$ ) of approximately constant density material and finally a region of slightly elevated average density (about $40 \AA$ ) immediately on top of the glass. Thus, for the purposes of modeling the nuclear response of the sample the electron density of the three layer model (figure 6.2) was used. The parameters used were: top layer $24.7 \AA, 51 \%$ bulk density; central portion $174.0 \AA$, $86 \%$ bulk density; rear section $36.6 \AA, 88 \%$ bulk density; substrate index*

\footnotetext{
* In fact, the angular zero for the data was not well determined (uncertaintly abour $0.2 \mathrm{mrad}$ ). If so, one might have been able to select one of the two fits in figure 6.3. However, based on how sensitive the fitting code was to initial conditions, one would still not trust the manylayer fit result to be an accurate reflection of realit.

- The information available about the substrate, from Niznhii Novgorod, was that it was "smooth glass". Measuring the density showed it to be $2.33 \pm .10 \mathrm{~g} / \mathrm{cc}$ and assuming the structure is pure $\mathrm{SiO}_{2}$ one obtains this value.
} 
$\delta=2.39 \times 10^{-6}+i 1.0 \times 10^{-8}$. The roughness at each interface was assumed to be Gaussian having width $\sigma=3.7 \AA$ (in fact, the fitting routine was allowed to vary the roughness at each interface independently, but the values found for each interface were nearly identical and thus set equal). The conversion from the index of refraction to density was done assuming the index of refraction of bulk density $(7.86 \mathrm{~g} / \mathrm{cc})$ iron at $14.4 \mathrm{keV}$ is $\delta=7.35 \times 10^{-6}+\mathrm{i} 3.35 \times 10^{-7}$. In practice, it was found that tradeoffs in the thickness and density values for the different layers were possible at the level of about $+3 \%$. Looking at the results in figure 6.3 , it is clear that the structure of the interface regions at the top and back of the layer can only be taken as approximate. The safe conclusions fitting the electronic response are that the density of the layer is reduced by about $14 \%$ from the bulk value, that there is an interfacial region of low density at the surface and a region of very slightly higher density at the rear of the layer.

Other parameters that should be considered with respect to a diffraction from a thin layer are the uniformity of the layer in the plane of the surface and the curvature of the substrate. However, in practice both of these parameters were neglected. The above measurement of the reflectivity (figure 6.1) was done with a $25 \mu \mathrm{m} \times 1 \mathrm{~cm}$ slit (vertical by horizontal, vertical scattering plane) defining the incident beam. During the measurement of the nuclear response, a larger vertical slit (about $0.5 \mathrm{~mm}$ ) was used. Figure 6.4 shows the electronic reflectivity measured with the large and small slits in the immediate vicinity of the first two interference minima, where the nuclear response was measured. The good agreement of the two measurements suggests that curvature and variations of the sample in the plane of the interface may be neglected. In addition, at various points in the fitting of the time response, described below, an angular integration range was included, but did not significantly improve the fits, and hence was finally dropped. 


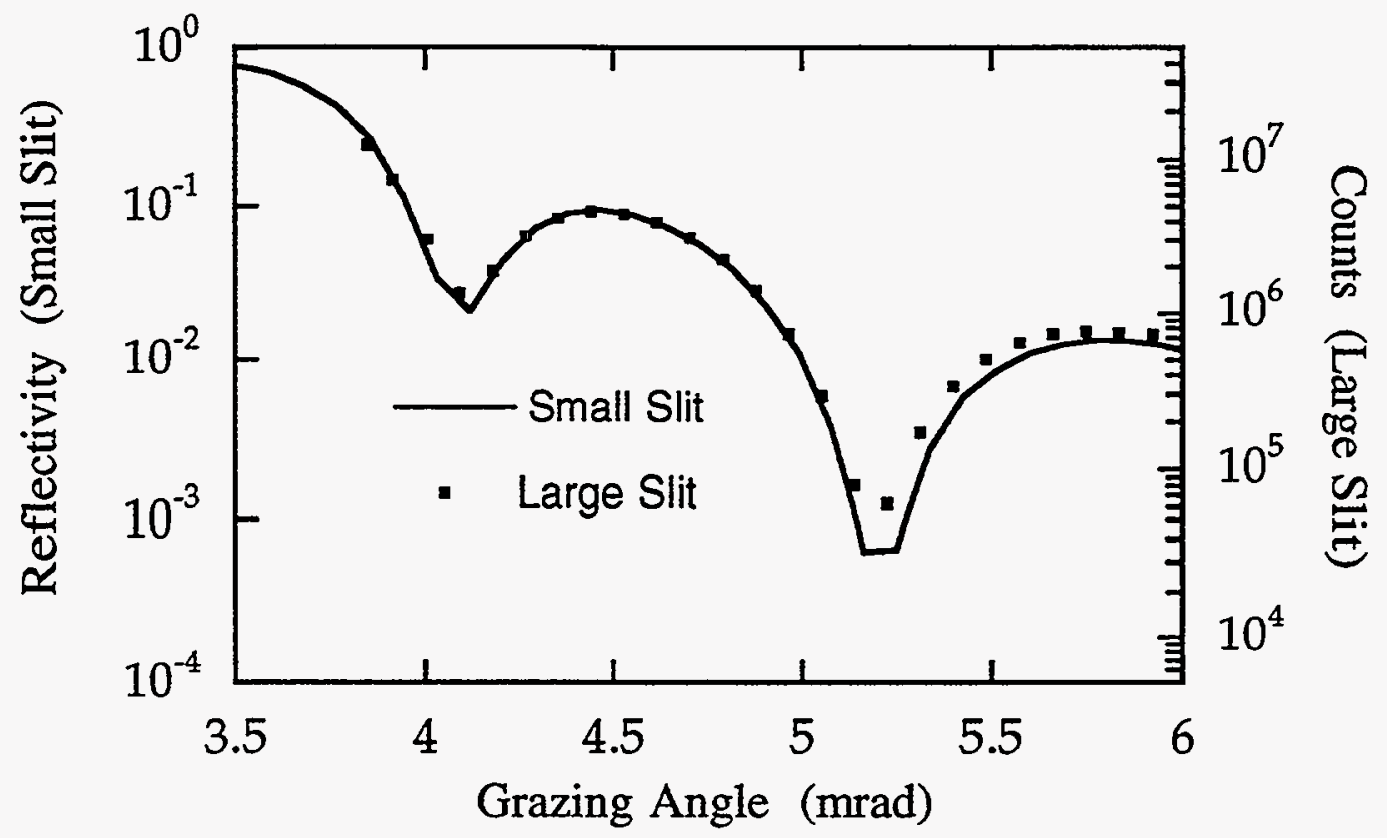

Figure 6.4. Comparison of the measured reflectivity profile with a small slit (as used in Fig. 6.1) and a large slit (as used at the time of the measurement of the nuclear response).

\section{Auger Analysis}

The models above used to calculate the $x$-ray reflectivity of the sample all assumed that the material was simply iron on glass, without any contamination of the iron by other elements. One might expect there to be at least some contamination of the surface with oxygen, an oxide layer. At the level of an x-ray reflectivity measurement, which essentially measures the electron density, an oxide layer is not readily distinguishable from reduced density iron. However, the nuclear response of an oxide, discussed below, is very different than that of pure iron.

Auger electron analysis was performed on a small piece of the iron layer to investigate its chemical composition. The peak-to-peak signals from the oxygen line at $503 \mathrm{eV}$ and the iron line at $703 \mathrm{eV}$ were measured as the surface of the sample was removed by sputtering with Xenon, providing the depth profile of the composition shown in figure 6.5. Unfortunately, the bombardment of the 
sample by the sputtering atoms tends to push atoms on the surface of the sample into the interior, thus limiting the resolution of this technique to $>\sim 20 \AA$.

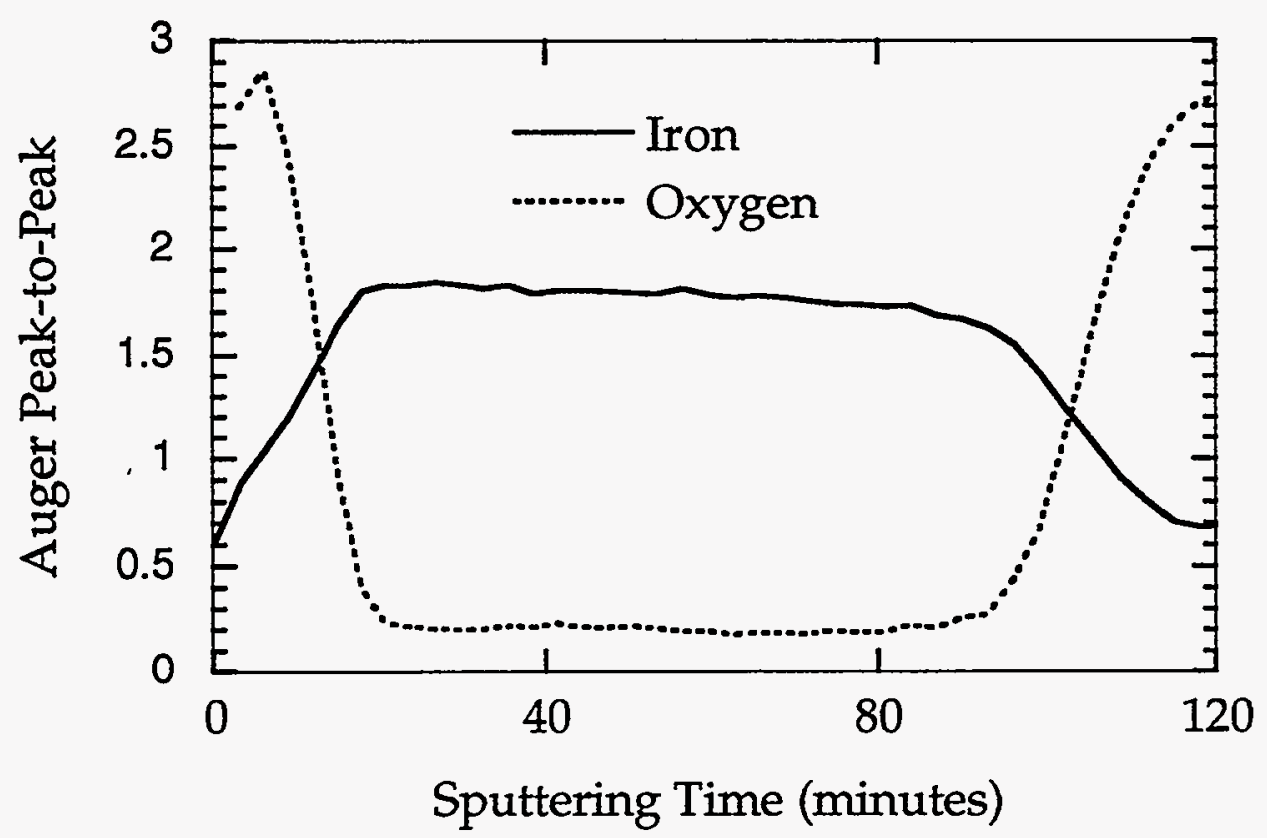

Figure 6.5. Sputter Auger-electron analysis of the iron layer. Assuming a constant sputtering rate gives a calibration of about 2.4 $\AA /$ minute from the $240 \AA$ thickness of the layer.

The sputter profile suggests that there is some oxygen contamination of the surface, but, practically it is very difficult to determine its depth. The oxygen signal reaches a minimum after about 20 minutes which corresponds to having removed about $50 \AA$ of material (where the sputtering rate, $2.4 \AA /$ minute, has been estimated by assuming a constant rate and using the known thickness of the layer). However, with the poor resolution, this is really a high upper bound for the oxygen contamination. One notes, for example, that the surface interface region is of comparable duration to the iron-glass interface at the back of the sample. Taking this as being about $8 \AA$ ( $=2.35 \mathrm{x}$ the rms. roughness from the electronic reflectivity fit) suggests that a guess of about $10 \AA$ for the surface oxide layer would not be unreasonable. However, the glass is an insulator, and thus the Auger profile, which relies on a conducting material, may be disturbed in its vicinity, so the reliability of this conclusion is not clear. 


\section{Conversion Electron Mössbauer Spectrum}

In principle, the nuclear response of the layer has more degrees of freedom than the electronic response, thus making the determination of the nuclear response solely from reflectivity measurements truly a difficult problem. However, significant help may be obtained by measuring the conversion electron Mössbauer spectrum (CEMS) of the sample. In this technique one measures the absorption based upon the number of internal conversion electrons that are produced. This allows absorption spectroscopy to be done on thin layers which would not sufficiently attenuate a transmitted beam or on samples that have thick backings. In general, CEMS is only sensitive to the surface of a sample (the electron escape depth is of order $0.25 \mathrm{um}$ ) but, for the $240 \AA$ sample used here, this does not matter. The thinness of the sample means that one may also safely neglect saturation effects that can lead to non-Lorentzian line shapes with thicker samples.

The conversion electron Mössbauer spectrum of the thin layer was measured at the Kurchatov Institute in Russia and the data made available to this author by A. I. Chumakov. The sample was placed in an electron detector (a gas filled proportional counter) and the number of conversion electrons measured as a function of the velocity of a radioactive source. Figure 6.6 shows both the spectrum measured from an iron foil and that measured from the iron layer. The response of the iron foil is very well fit ${ }^{*}$ by a simple sextet of Lorentzian lines of width $2.7 \Gamma_{0}$, which includes the source width of $1.7 \Gamma_{0}$, thus establishing a good control. The response of the iron layer shows somewhat broader lines and traces of another component that raises the background between the peaks.

\footnotetext{
The fit was preformed by A.I. Chumakov. It includes the effects of saturation.
} 


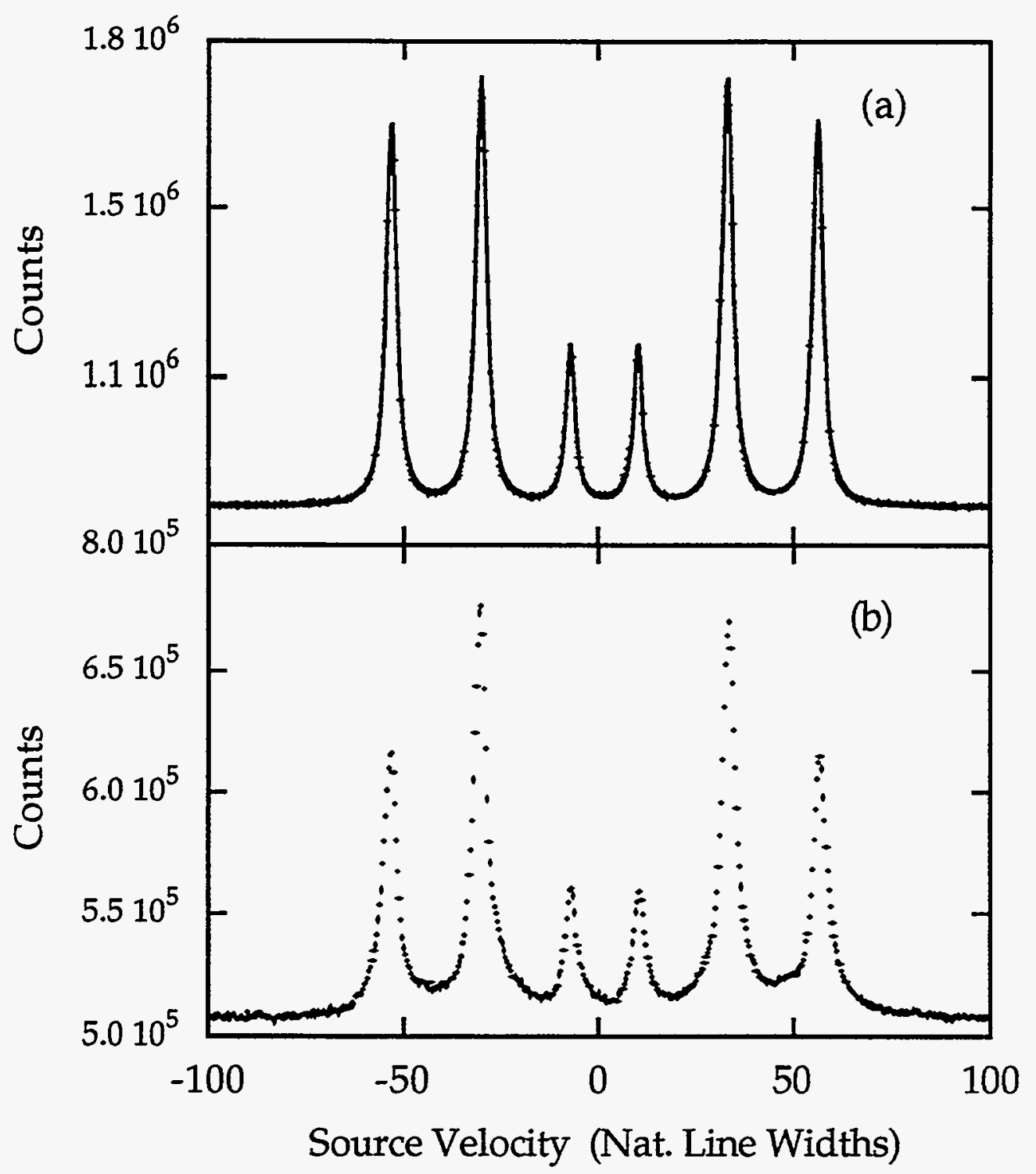

Figure 6.6 Conversion electron Mössbauer spectra from a 95\% enriched ${ }^{57} \mathrm{Fe}$ foil (a) and the thin iron layer (b). The solid line in (a) is a fit assuming a simple six line response from the iron foil. 


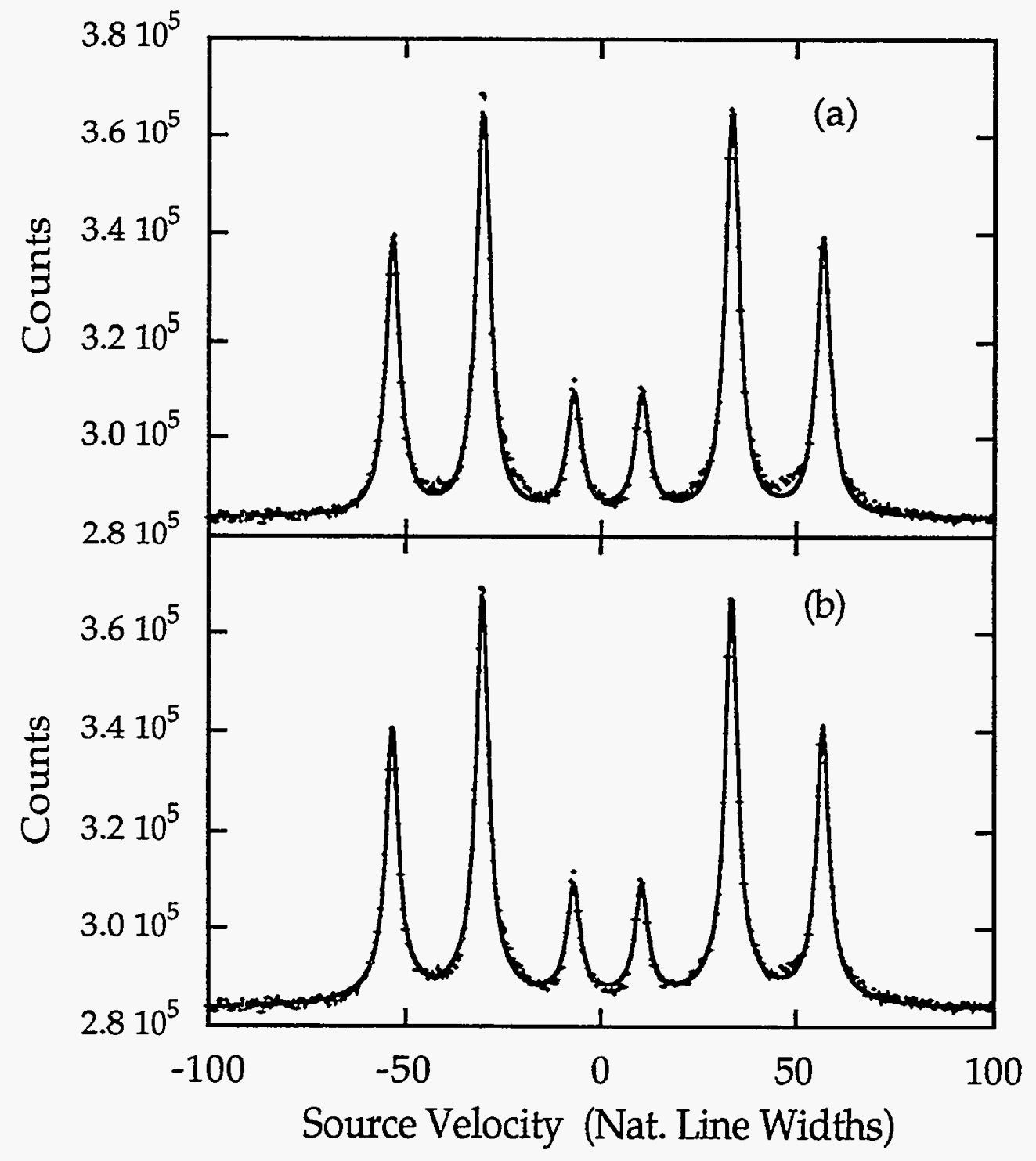

Figure 6.7. Fits to the iron layer conversion electron spectrum. The fit shown in (a) uses a single sextet of lines, width $2.6 \Gamma_{0}$, and has chi-squared/point $=4.8$ while that in (b) uses two sextets having widths 2.0 and $14.2 \Gamma_{0}$, improving the chi-squared/point to 2.5 .

Figure 6.7 shows two fits to the CEMS of the iron layer* . The first uses a single sextet of Lorentzian lines determined to have width $2.6 \Gamma_{0}$ (where the

\footnotetext{
* An astute reader may note that the vertical scales in figure $6.6 \mathrm{~b}$ and figure 6.7 are not the same. The conversion electron spectrum of the thin layer was measured twice, with about 6 months intervening. The second measurment showed essentially the identical response to the first, but was done to larger velocities $\left( \pm 150 \Gamma_{0}\right)$ and thus better for fitting the baseline (though the additional velocity range is not shown).
} 
source width has been removed) and positions identical to that of the iron lines. The second fit (b) included an additional sextet that was broadened. In this case, the width of the narrow component was determined to be $2.0 \Gamma_{0}$ and the width of the broader component $14.2 \Gamma_{0}$. The locations of both sets of lines was that of the lines from the iron foil. The chi-squared / point for the fit with a single sextet was 4.8 while that for the double sextet 2.5 , so we find it necessary to fit the conversion electron data with two sextets, as did Isaenko et al,[Isaenko, et al., $1994,9]$ in their work with this sample.

The results of the fit here differ in one significant way from the work by Isaenko, et al .: here we find that the broad component comprises $34 \pm 8 \%$ of the total number of resonant nuclei, while Isaenko, et al, find it is only $17 \pm 3 \%$. No reason for this discrepancy has been discovered. However, since the fits to the time response seem to be better with the higher fraction, and since this author has checked the fits presented here most carefully, the higher percentage of broadened component is retained below.

\section{Oxide Layers and the CEMS Data}

At the level of this CEMS data, there is no hint of any oxide contamination of the iron layer, despite the results from the Auger analysis, and despite what "common sense" might say about a sample left in room air for months at a time. However, at the level of the CEMS data, this is quite unambiguous. Iron oxides, in general, will have a different hyperfine splitting than iron, with the nuclear magnetic field increased by a factor of about 1.3 to 1.6, depending on the oxide (see table 10.1 of [Greenwood and Gibb, 1971, 11], p241). Thus, an oxide layer should appear as additional lines at larger velocities in the conversion electron spectrum. However, allowing the fit to include lines where the oxide might appear gave negligible improvement in the chi-squared, and amplitudes of the lines consistent with zero. Thus, there is no evidence in the conversion electron spectrum for an oxide layer. Any oxide layer must be either very small, or have a low resonant fraction, or perhaps have an extremely broad line width. 
One might also wonder about the possibility of the sample changing with time. However, the CEMS measurement was repeated after the Auger measurement above, with the identical result.

The simplest conclusion is that only a small portion of the iron in the layer is in oxide form, too small to be detected above background in the CEMS. The total external reflection measurement of Isaenko et al [Isaenko, et al., 1994, 9] on this sample seems to support this conclusion. This study, done with a radioactive Mössbauer source, clearly showed the presence of an additional line at about $77 \Gamma_{0}$ when the angle of incidence was reduced to $3.2 \mathrm{mrad}$ (it did not appear at higher angles). They interpreted this as belonging to a small amount ( $1 \%$ of the total iron content of the sample) of hematite $\left(\alpha-\mathrm{Fe}_{2} \mathrm{O}_{3}\right)^{+}$.

Another possible explanation for these results is that the resonant fraction of the oxide might be reduced, or (nearly equivalently) the width of the lines may be so broad as to not appear in the CEMS. In fact this suggestion was once made based on the investigation of an iron sample [Belozerskii, et al., 1982, 17] . The conversion electrons from the sample were energy analyzed, in order to give information about the depth at which they had been emitted. The conclusion that there was a layer of low resonant fraction at the surface relied on distinguishing the energy differences between electrons from the surface and from $25 \AA$ below the surface. In addition, none of the Mössbauer spectra used to draw these conclusions are presented. Thus it is a little difficult to asses the certainty of the conclusion. There is also extensive work with monolayer ${ }^{57} \mathrm{Fe}$ probes used with $56 \mathrm{Fe}$ surfaces or multilayers, [Droste, et al., 1986, 18, Korecki and Gradmann, 1986, 19, Liu and Gradmann, 1993, 20] and, although the conditions are typically somewhat better (i.e. the samples are kept in UHV or covered with cap layers) there is no suggestion of any reduction in the resonant fraction near a surface.

\footnotetext{
+ In fact, the field strength was reduced from that of hematite, making it more consistent with magnetite, $\mathrm{Fe}_{3} \mathrm{O}_{4}$ [Daniels and Rosencwaig, 1969, 12] .which has been suggested by some authors as being the oxide most typically found on iron surfaces[Stockbridge, et al., 1961, 13] , [Langell and Somorjai, 1982, 14] [Stadnik, et al., 1989, 15, Kim and Olivieria, 1993, 16]
} 


\section{Time Response Measurements}

The time evolution of the specularly reflected beam from the thin iron layer was measured at the Cornell High Energy Synchrotron Source (CHESS). The experimental setup was as described in chapter 2, with a nested asymmetric (4 2 2) / symmetric (10 64) monochromator [Toellner, et al., 1992, 21] providing a $\mathrm{x}$-ray beam of bandwidth about $10 \mathrm{meV}$ at the $14.4 \mathrm{keV} 57 \mathrm{Fe}$ nuclear resonance energy. The iron layer was mounted on the sample stage of a Huber 4-circle diffractometer and an avalanche diode from Advanced Photonix Inc. (API) was used as a detector on the $2 \theta$ arm.

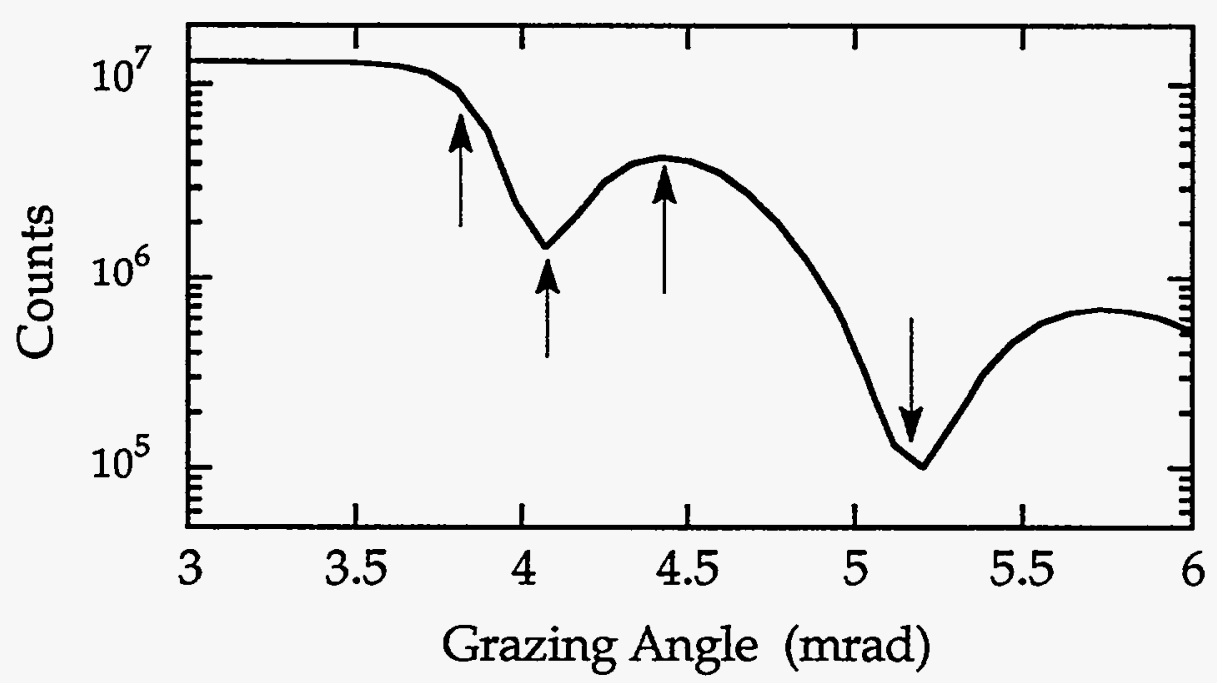

Figure 6.8 Angular locations for measurement of the time response of the thin iron layer relative to the electronic rocking curve.

The time response was measured for several angles of incidence and with two orientations of an external magnetic field (about 300 oersted). This field was either parallel or perpendicular to the scattering plane, and was always in the plane of the sample. Various parameters for each measurement are listed in table 6.1, while figure 6.8 shows the approximate locations of the measurement points relative to the electronic (prompt) reflectivity curve. Figures 6.9 and 6.10 show the time responses measured in each field configuration. 


$\begin{array}{ccccc}\text { Notation } & \begin{array}{c}\text { Alignment } \\ \text { Field }\end{array} & \begin{array}{c}\text { Approx. } \\ \text { Angle } \\ \text { (mrad) }\end{array} & \begin{array}{c}\text { Prompt } \\ \text { Rate } \\ \text { (kcts/sec) }\end{array} & \begin{array}{c}\text { Delayed } \\ \text { Rate } \\ \text { (cts/sec) }\end{array} \\ 110 & \text { Parallel } & 3.8 & 1200 & 30 \\ 111 & \text { Parallel } & 4.05 & 200 & 25 \\ 112 & \text { Parallel } & 4.35 & 430 & 20 \\ 113 & \text { Parallel } & 5.15 & 8 & 10 \\ \text { Pp1 } & \text { Perp. } & 4.05 & 230 & 25 \\ \text { pp2 } & \text { Perp. } & 4.35 & 340 & 10 \\ \text { Pp3 } & \text { Perp. } & 5.15 & 13 & 15\end{array}$

Table 6.1. Various parameters during the measurements of the time response of the thin iron layer. The direction of the alignment field (and hence the magnetic axis) is relative to the scattering plane.

\section{Kinematic vs. Dynamical Characteristics of the Time Response}

The measured time response can be thought of as resulting from both kinematic and dynamical scattering, and it is convenient to discuss these aspects independently. Some of the distinctions between kinematic and dynamical scattering were discussed in chapter 5, however, it is useful to re-consider these two types of scattering with the time response in mind.

The key point in this context is that kinematic scattering is linear in the scattering amplitude. Returning to equation (5.2) one notes that it breaks up into the product of a geometric term and the scattering amplitude, with all the frequency dependence appearing in the scattering amplitudet. If the frequency dependence of the scattering amplitude does not change with location in the sample, integration of (5.2) over the sample volume will always yield the same frequency response, up to a geometric scale factor. Thus, although the amount of scattered radiation may depend on the geometry, the shape of the frequency

t Stricly speaking, the magnitude of the wave vector depends upon the frequency as well, but this dependence is negligible for the cases considered here. 
response and that of the time response will not change, in a kinematic scattering limit with a uniform sample. Furthermore, the frequency/time response in this limit directly reflects the microscopic properties of the scattering amplitude.

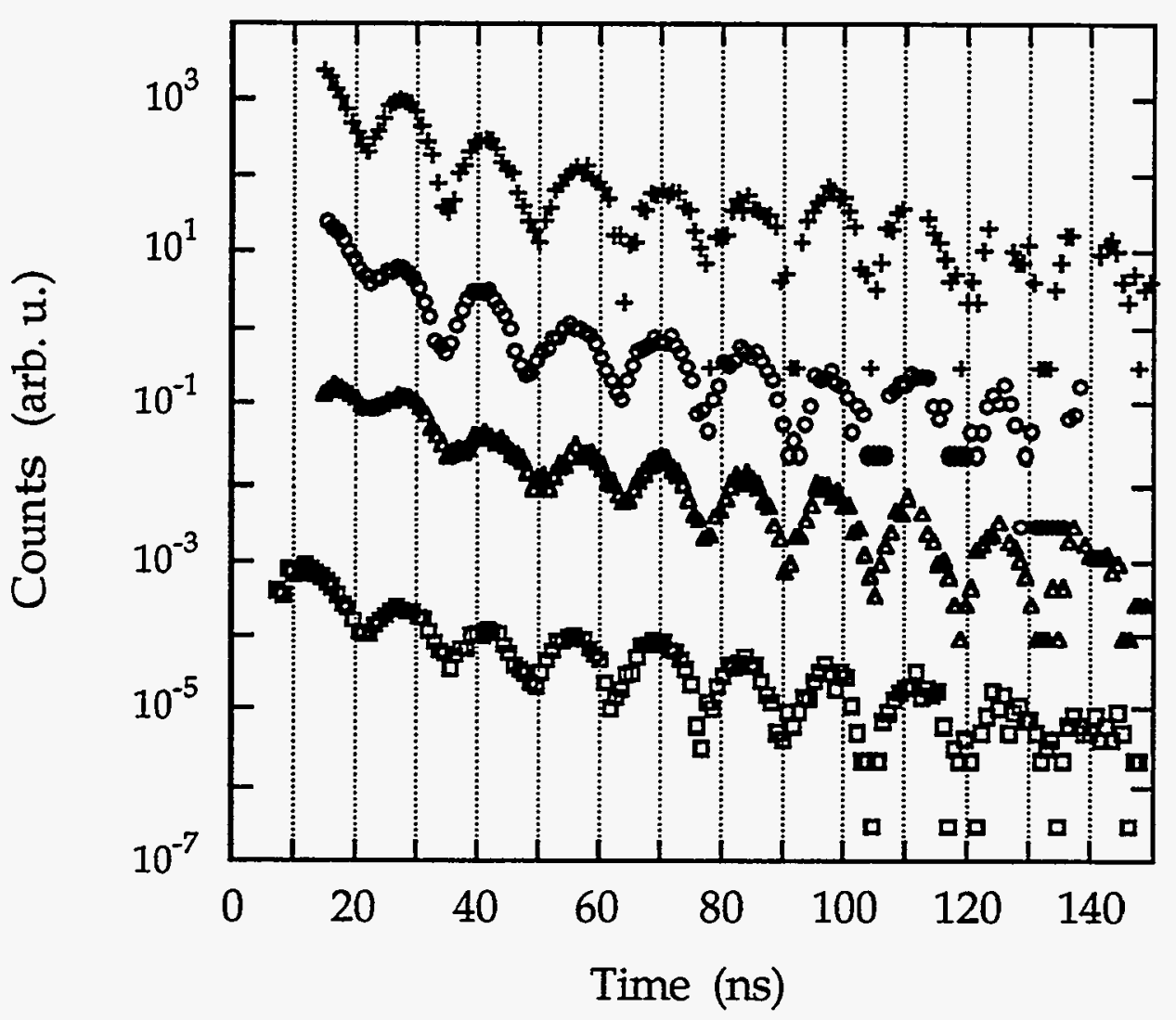

Figure 6.9 Time evolution of the specularly scattered radiation after excitation by the synchrotron pulse at $t=0$. The external alignment field was in the scattering plane (parallel to the photon propagation direction). Each trace corresponds to a different angle of incidence, and from top to bottom these are $110,111,112$ and 113 (see table 6.1). The vertical scale for each (after the first) has been reduced by two orders of magnitude.

Inspection of figures 6.9 and 6.10 show that there are changes in the time response of the layer at different angles. This indicates that dynamical effects are important and/or the frequency dependence of the scattering amplitude of the 
iron is not homogeneous. In fact, total external reflection is a dynamical scattering phenomenon and so for the small grazing angles used here, the appearance of dynamical effects is not surprising. However, some of the essential characteristics of the time response may be explained from a kinematic standpoint.

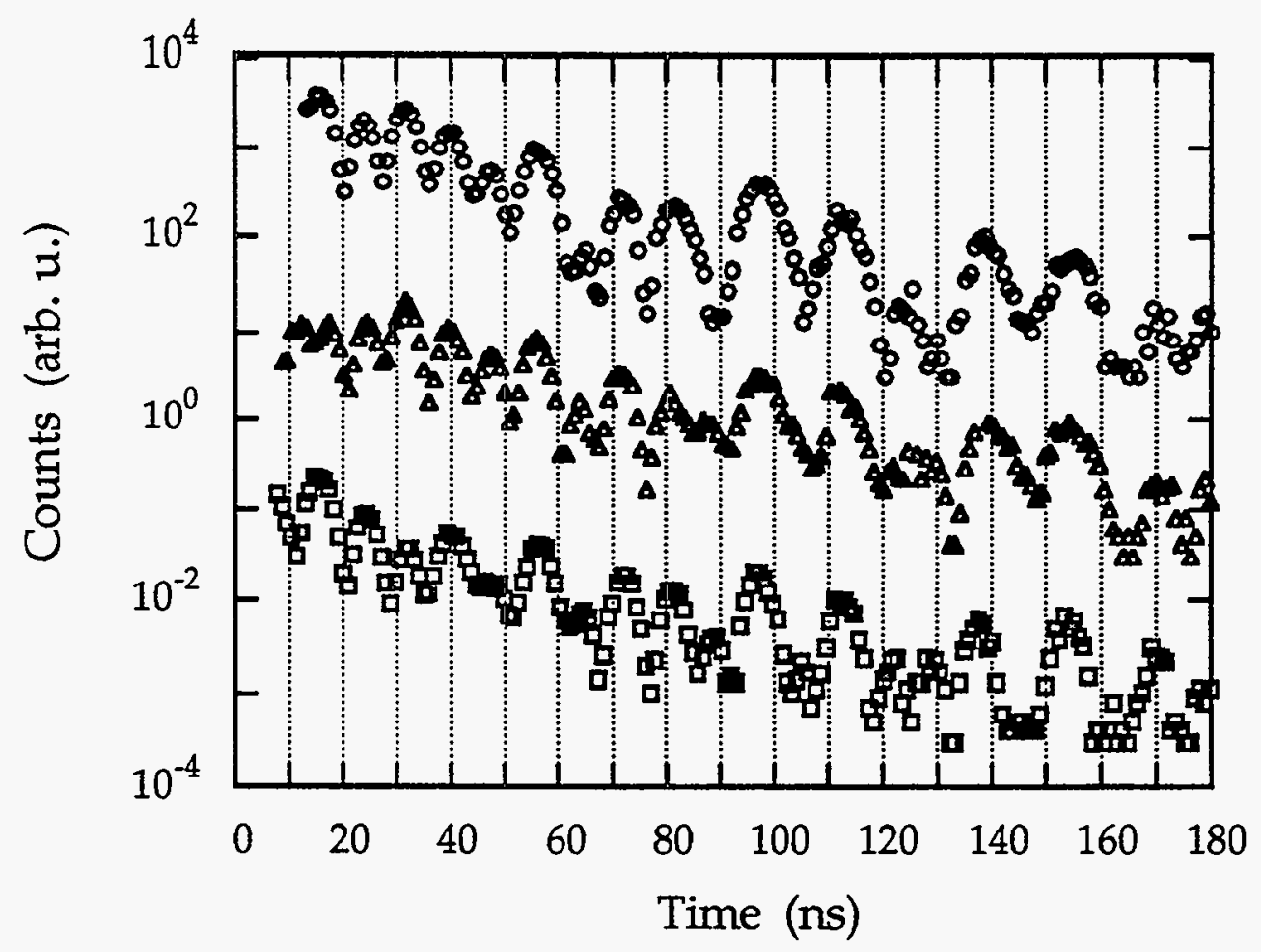

Figure 6.10 Time evolution of the specularly scattered radiation after excitation by the synchrotron pulse at $t=0$. The external alignment field was perpendicular to the scattering plane. Traces corresponds to a different angles of incidence, and from top to bottom these are pp1, pp2 and pp3 (see table 6.1). The vertical scale for each (after the first) has been reduced by two orders of magnitude.

Kinematic Time Response: Quantum Beats

In a kinematic limit, the phase and amplitude of the scattered wave directly reflect that of the microscopic scattering amplitude. This is sufficient to 
explain some of the differences between the time responses observed in figure 6.9 and those in 6.10. Instead of discussing the scattering amplitude directly, it is convenient to discuss the index of refraction of the sample, as this is the parameter most easily related to grazing incidence measurements with thin films*. $\delta=1-n$ is just linearly related to the scattering amplitude through the Lorentz relation. This is discussed in chapter 4 , and the relevant relationship is reproduced here for convenience:

$$
\delta=-\frac{2 \pi}{k^{2}} N F=-\frac{2 \pi}{k^{2}} N\left(f_{r} F_{n}-r_{e} f_{e}\right)
$$

The various quantities in (6.1) are the wave vector $k=2 \pi / \lambda$, the number density of atoms, $N$, and the scattering amplitude $F$. $F$ has both nuclear and electronic components: $F_{n}$ is the nuclear component discussed in appendix $A$ while $-r_{e} f_{e}$ is the electronic component $\left(\mathrm{f}_{\mathrm{e}}\right.$ is in units of electrons and $\mathrm{r}_{\mathrm{e}}$ is the classical radius of the electron). The parameter $f_{r}$ is the resonant fraction of nuclei (the fraction of nuclei showing the Mössbauer effect), sometimes called the Lamb-Mössbauer factor (or, incorrectly, the Debye-Waller factor) and for polycrystalline iron at room temperature this is approximately 0.77 [Bergmann, et al., 1994, 22]. Of course, if more than one type of scatterer is present in the sample, a sum should be carried out over the appropriate number densities and scattering amplitudes. In the event of complicated polarization dependence, (6.1) is taken to hold only for the eigenpolarizations.

The essential change in the beat patterns between figures 6.9 and 6.10 may be traced directly to the kinematic interaction of the linearly polarized synchrotron radiation with the nuclear spins aligned in the magnetic field. In iron metal, the field at the nucleus is known to be antiparallel to the domain magnetization [Hanna, et al., 1960, 23], and in thin films, the magnetization is primarily within the plane of the film (see, e.g. [Prutton, 1964, 24]). Thus, since the scattering angles are always small for this work ( $2 \theta<\sim 10 \mathrm{mrad})$, one can determine the basic beat patterns by considering the polarization selection rules for forward scattering with the nuclear magnetic field parallel to the $x$-ray

\footnotetext{
* The term "index of refraction" is used here interchangably with the decrement of the index of refraction from $1, \delta=1-n$, as they contain the same information. This will frequently be the case, though plots will always be of $\delta$, since this is the small quantity that varies.
} 
propagation direction or parallel to the $\mathrm{x}$-ray polarization (electric field) direction (these are the 11 and pp cases, respectively). The fact that the field is antiparallel (instead of parallel) to the external field is of no concern in this work, though for finite scattering angles, the (signed) direction of the field may be important and can lead to interesting effects [Brown, et al., 1992, 25]

Figure 6.11 shows the indices for the eigenpolarizations in the 11 case. The eigenpolarizations are left and right hand circularly polarized light, which are scattered by the $\Delta \mathrm{m}= \pm 1$ transitions. The $\Delta \mathrm{m}=0$ transitions are not excited. The index shown in 6.10 is appropriate for iron of 0.86 bulk density and with resonant fraction, $\mathrm{f}_{\mathrm{r}}=0.75$. In accordance with the results from CEMS discussed above, $34 \%$ of the iron atoms were assumed to have a line width of $14.2 \Gamma_{0}$ and $66 \%$ to have a width of $2.0 \Gamma_{0}$. One notes that the nuclear contribution actually pushes $\operatorname{Re}\{\delta\}$ less than zero, which is not typically the case for electronic scattering at any energy (though see [Smith and Barykoumb, 1990, 26] for possible exceptions in Si near the L-edges). 


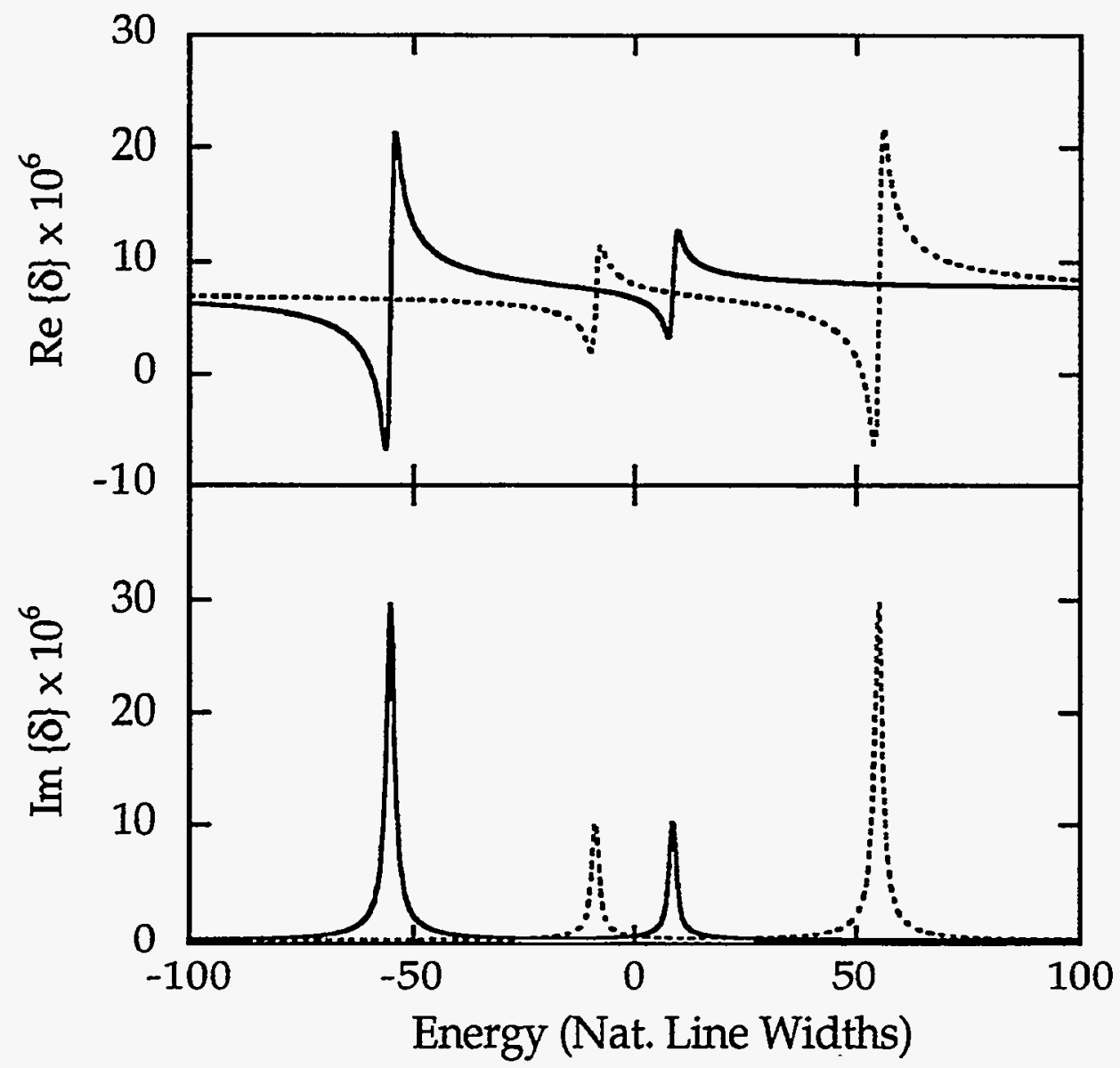

Figure 6.11. Index of refraction for the eigenpolarizations in iron with an alignment field parallel to the x-ray propagation direction.

Figure 6.12 shows the magnitude squared of the Fourier transform of the index of refraction for a sample with the magnetic axis parallel to the photon propagation direction. The temporal intensity distribution is independent of the photon polarization so both eigenpolarizations give the same time response. The dots in the figure are just a reproduction of the measured response, 113, shown in figure 6.9 The period of the beats in the calculation matches that of the data, but the details of the time response are somewhat different. Most notably, the decay rates are not the same, and, interestingly enough, the modulation of the data is greater than that of the calculation, exactly the opposite of what one would expect given concerns of detector resolution. Both of these differences are due to dynamical scattering effects and will be discussed below. It is easy to see from 
figure 6.9 that the other responses measured at smaller grazing angles will deviate more from the kinematic response in figure 6.12. This is because dynamical effects become more pronounced at smaller grazing angles.

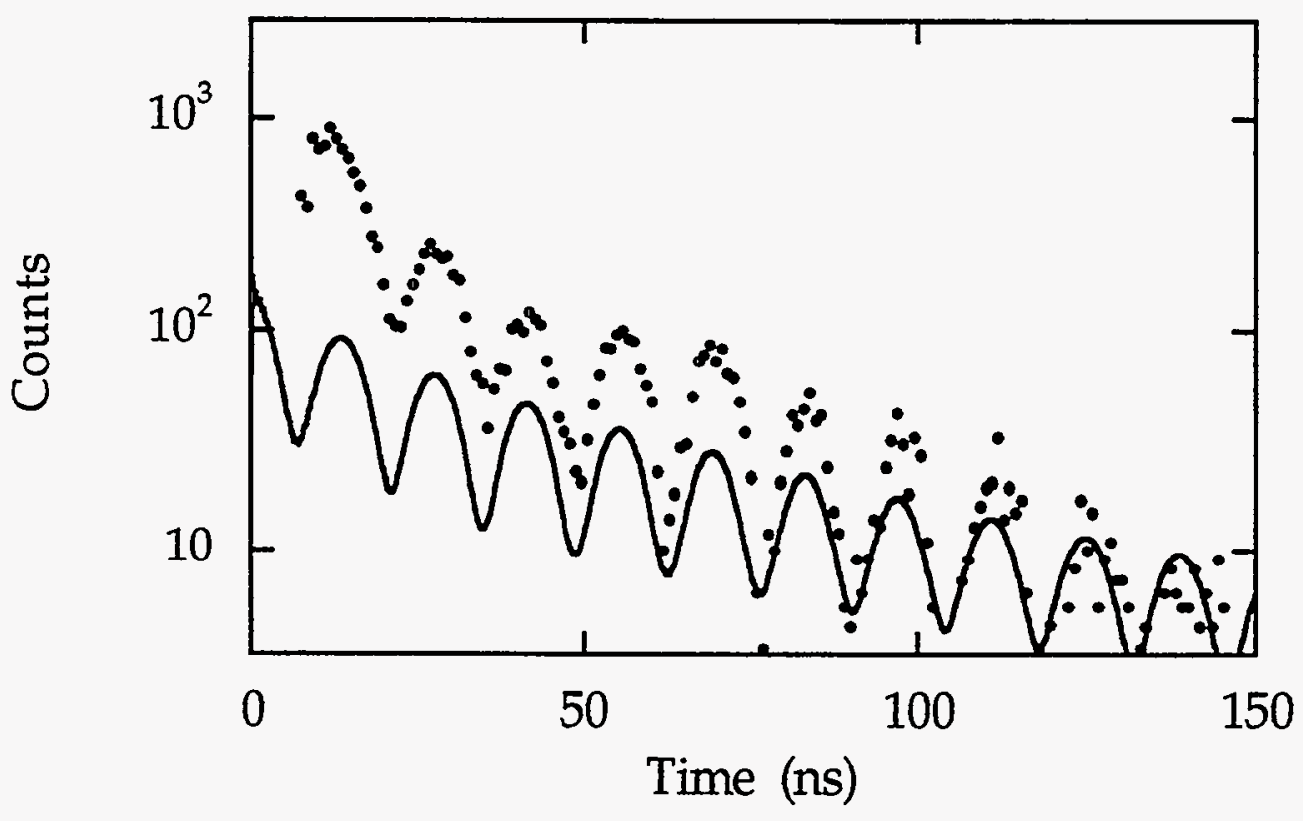

Figure 6.12 Square of the Fourier transform of the index of refraction shown in figure 6.11 (parallel field direction). The temporal intensity distribution is independent of the polarization of the $x$-ray. The points are the time response of the layer measured in the second interference minimum, 113 .

Figures 6.13 and 6.14 are completely analogous to 6.11 and 6.12, but for the case with magnetic field perpendicular to the direction of propagation of the $x$ rays. Here the eigenpolarizations are linear: one polarization (electric field vector parallel to the quantization axis) will excite the $\Delta m= \pm 1$ transition while the other (electric field perpendicular to the quantization axis direction) will excite only the $\Delta \mathrm{m}=0$ transitions. Figure 6.14 shows the time response for only the first case, as this is appropriate to the experimental setup used (vertical scattering plane, horizontal incident polarization and horizontal alignment field). Again, the time response at the larger angle (pp3), resembles the Fourier transform of the scattering amplitude in its gross features, but differs in the details. 


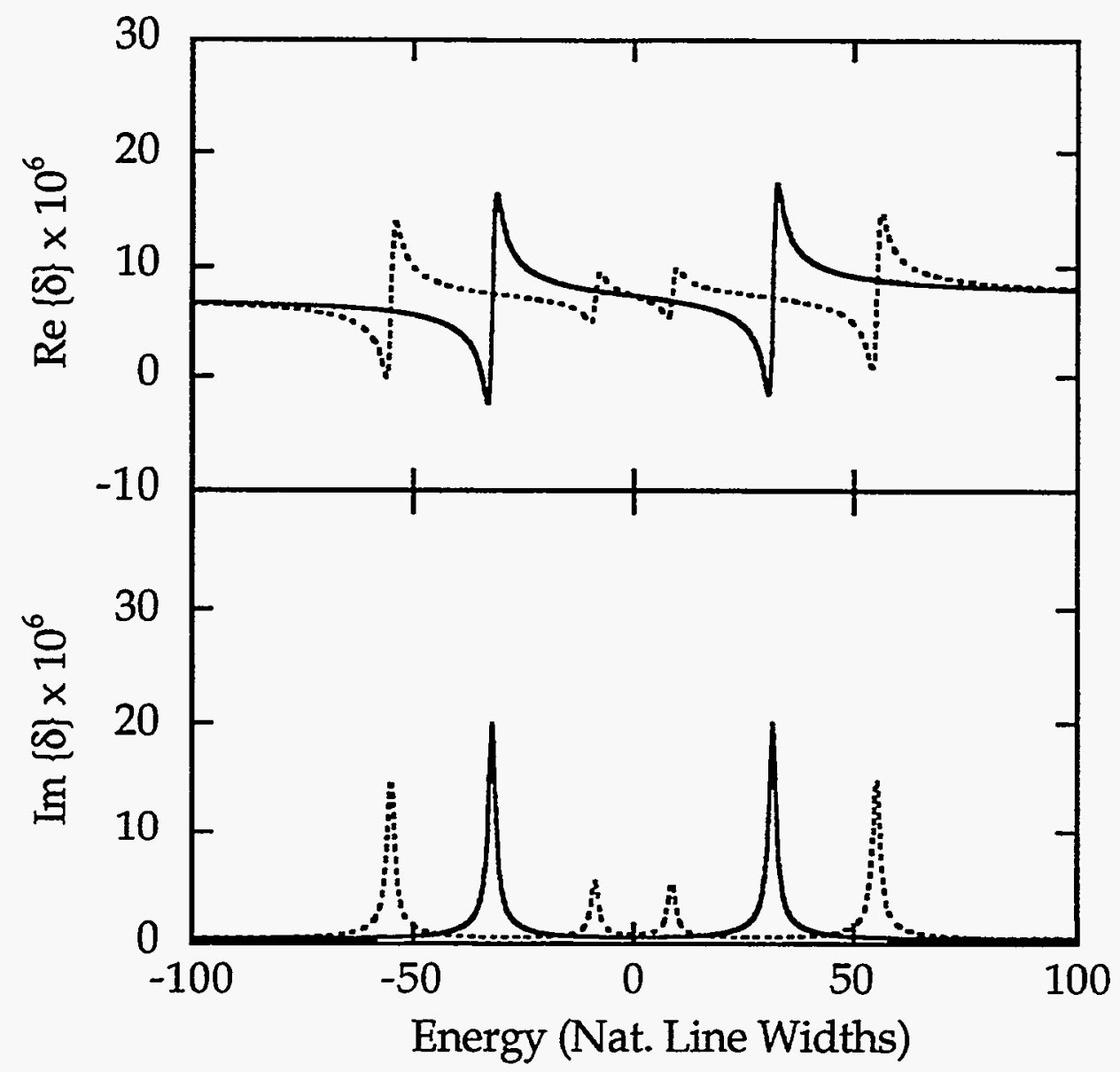

Figure 6.13. Index of refraction for the eigenpolarizations in iron with an alignment field perpendicular to the $x$-ray propagation direction. 


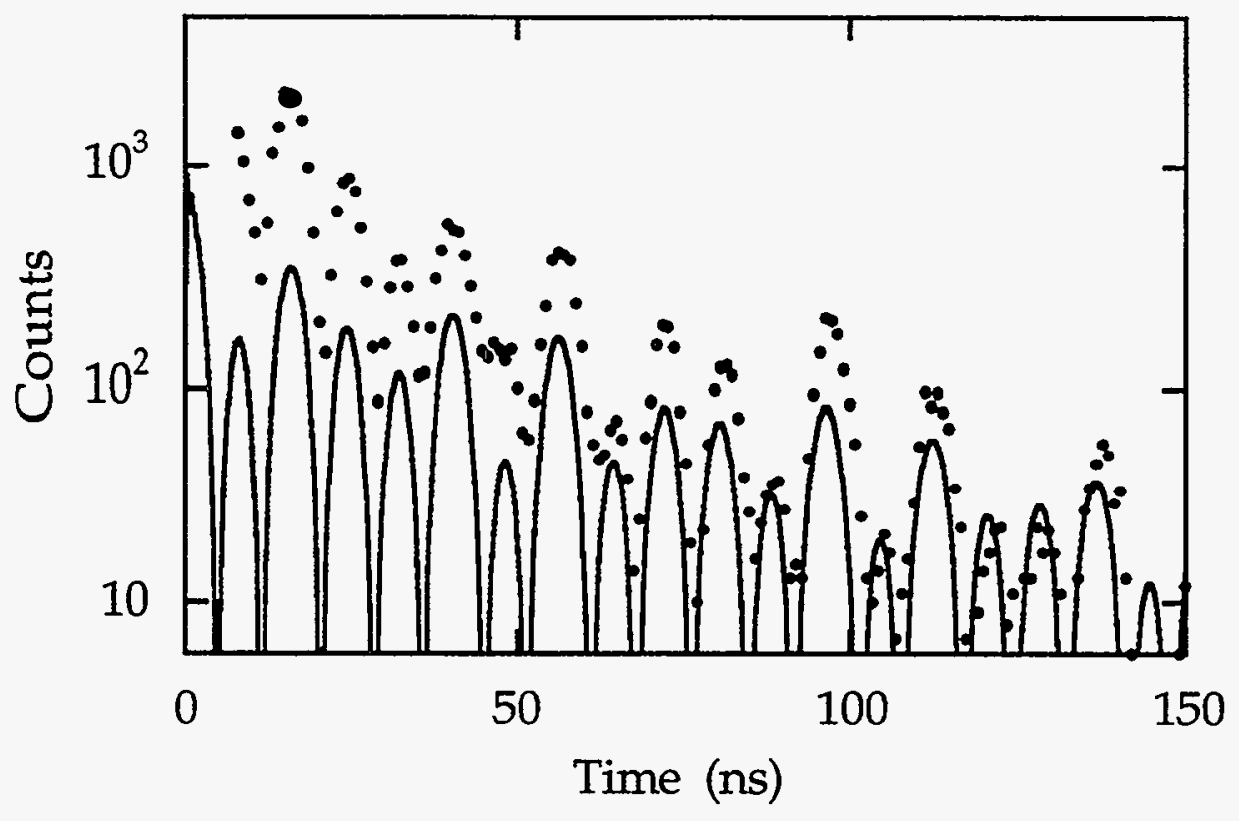

Figure 6.14 Square of the Fourier transform of the index or refraction shown in figure 6.13 (perpendicular field direction). Only one eigenpolarization is shown. The points are the time response of the layer measured in the second interference minimum, pp3.

\section{Dynamical Effects}

Dynamical effects in nuclear scattering have been discussed by many authors ([Trammell, 1961, 27],[Kagan, et al., 1968, 28],[Hannon and Trammell, 1969, 29],[Kagan, et al., 1979, 30, van Bürck, et al., 1980, 31]), largely in the context of diffraction from perfect crystals, though forward scattering [Kagan, et al., 1979, 30] and scattering from thin films [Hannon, et al., 1985, 32] have been discussed as well. The relevant effects in this case can be divided into 3 categories: coherent enhancement or speedup of the scattering at grazing incidence, effects due to the thin layer nature of the sample, and, modification of the quantum beat structure due to dynamical effects. Each of these will be discussed in turn.

Coherent enhancement in resonant scattering has been discussed in chapter 2 in some generality. Here we investigate it in the context of grazing 
incidence near the electronic critical angle of a semi-infinite sample: the effects of the thin layer nature of the sample will be discussed separately. Also, for the moment, we neglect polarization effects and assume an unsplit line. This investigation is computational, since this author was not able to derive an analytic expression for the time response in this case, though other authors have done so when the electronic scattering into the reflected beam may be neglected ([Kagan, et al., 1979, 30] [Hannon, et al., 1985, 32]) Electronic scattering must be included here.

The reflected amplitude for $\mathrm{x}$-rays incident on a planar vacuum-material interface can be described by the Fresnel reflection coefficient discussed in the chapter 4:

$$
\begin{aligned}
& R_{\theta}(\omega)=\frac{1-\beta}{1+\beta} \\
& \beta=\left[1-2 \delta(\omega) / \theta^{2}\right]^{1 / 2}
\end{aligned}
$$

The decrement of the index of refraction from unity is just the sum of nuclear and electronic parts and has the form

$$
\begin{aligned}
\delta(\omega) & =\delta_{\mathrm{n}}(\omega)+\delta_{\mathrm{e}} \\
& =\frac{\delta_{\mathrm{n} 0}}{2 \hbar\left(\omega-\omega_{0}\right) / \Gamma+\mathrm{i}}+\delta_{\mathrm{e}}
\end{aligned}
$$

Where $\hbar \omega_{0}$ is the resonance energy and $\Gamma$ is the resonance line width.

Combining equations (6.1) and (A.17) gives for an un-split resonance

$$
\delta_{n 0}=\pi \frac{N}{k^{3}} \frac{2 j_{e}+1}{2 j_{g}+1} \frac{\Gamma_{r}}{\Gamma} f_{r}
$$

$\mathrm{N}$ is the number density of resonant nuclei, $\mathrm{k}$ is the wave vector, $\mathrm{j}_{\mathrm{e}}$ and $\mathrm{j}_{\mathrm{g}}$ are the excited and ground state nuclear spins and $\Gamma_{\mathrm{r}}$ is the radiative line width of the transition. 
Figure 6.16 shows the reflectivity of a sample, calculated from (6.2), as a function of angle near the electronic critical angle. The index of refraction for this case is shown in figure 6.15 and corresponds to a transition of width $\Gamma=2 \Gamma_{0}$ and amplitude, $\delta_{n 0}=2.5 \times 10^{-5}$, comparable with one of the stronger lines for the iron sample (see figures 6.11 and 6.13). The non-resonant contribution to the index is just that appropriate for iron of density $6.76 \mathrm{~g} / \mathrm{cc}$ ( $86 \%$ of bulk density) at 14.4 $\mathrm{keV}: \delta_{\mathrm{e}}=6.32 \times 10^{-6}+\mathrm{i} 2.88 \times 10^{-7}$ The electronic critical angle in this case is 3.55 mrad.

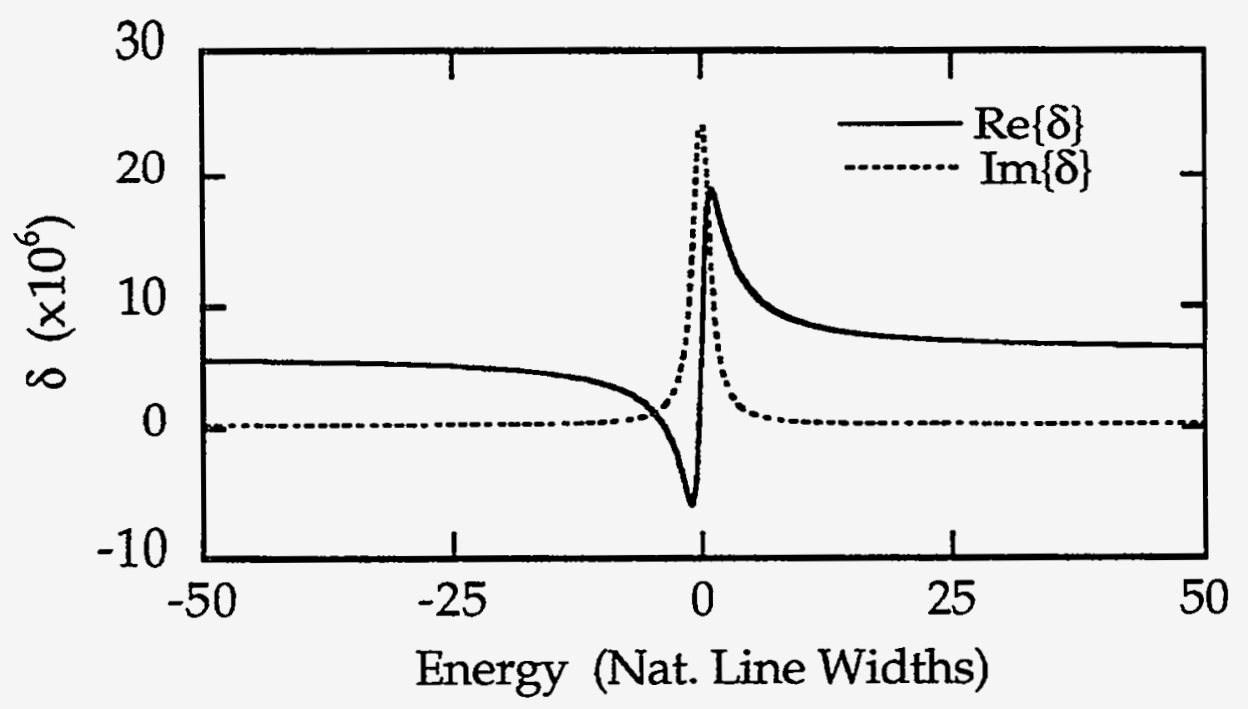

Figure 6.15. Index of refraction used for single line calculations. Note that here, as throughout this thesis, the magnitude of the imaginary part is plotted. Strictly speaking, to agree with the plane wave definition used in chapter 4, the imaginary part is negative. 


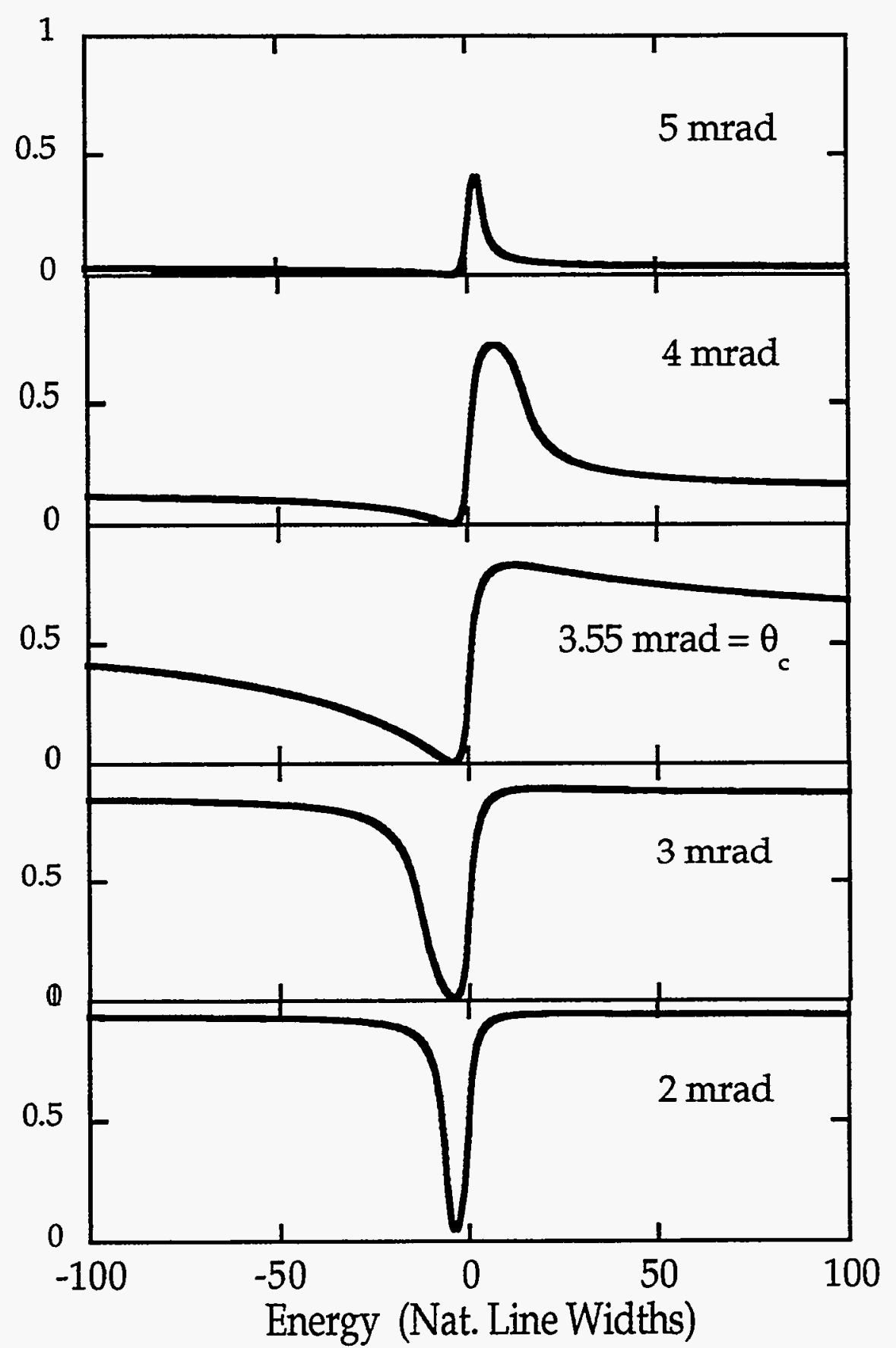

Figure 6.16. Grazing incidence reflectivity as a function of energy for a material with a single resonant transition (index of refraction shown in figure 6.15).

The basic features of the reflectivity curve were discussed by Bernstein and Campbell in 1963[Bernstein and Campbell, 1963, 1]. At frequencies below the nuclear resonance frequency the phase shift $\left(\operatorname{Re}\left\{\delta_{n}\right\}\right)$ from the nuclear 
resonance cancels that from electronic scattering and there is decreased reflectivity, while at frequencies just above the transition, the two components (nuclear and electronic) add and the reflectivity is enhanced. The appearance of either a peak or a trough is then governed by the background electronic scattering.

The time evolution of the specularly reflected wave after pulse excitation is just the Fourier transform the frequency response:

$$
G_{\theta}(t)=(2 \pi)^{-1} \int_{-\infty}^{+\infty} R_{\theta}(\omega) e^{i \omega t} d \omega
$$

In addition, we note that

$$
G_{\theta}(t>0)=(2 \pi)^{-1} \int_{-\infty}^{+\infty}\left[R_{\theta}(\omega)-R_{\theta}^{e}\right] e^{i \omega t} d \omega
$$

where $R_{\theta}^{e}=R_{\theta}(\omega \rightarrow \infty)$ is just the reflectivity in the absence on nuclei. Thus for the purposes of estimating the time response after the pulse excitation, it is more convenient to plot the square of the difference $\left|R(\omega)-R_{\theta}^{e}\right|^{2}$. This is shown in figure 6.17 and can be regarded as the frequency spectrum of the delayed response. Evidently the frequency response broadens as the critical angle is approached from either larger or smaller angles. This is somewhat related to the tendency of the frequency response to broaden as the exact Bragg position is approached for a finite order pure nuclear Bragg reflection [van Bürck, et al., 1980,31 ] (specular reflection is sometimes referred to as the zeroth order Bragg peak). However, there are also some subtle differences, as will be discussed later. 


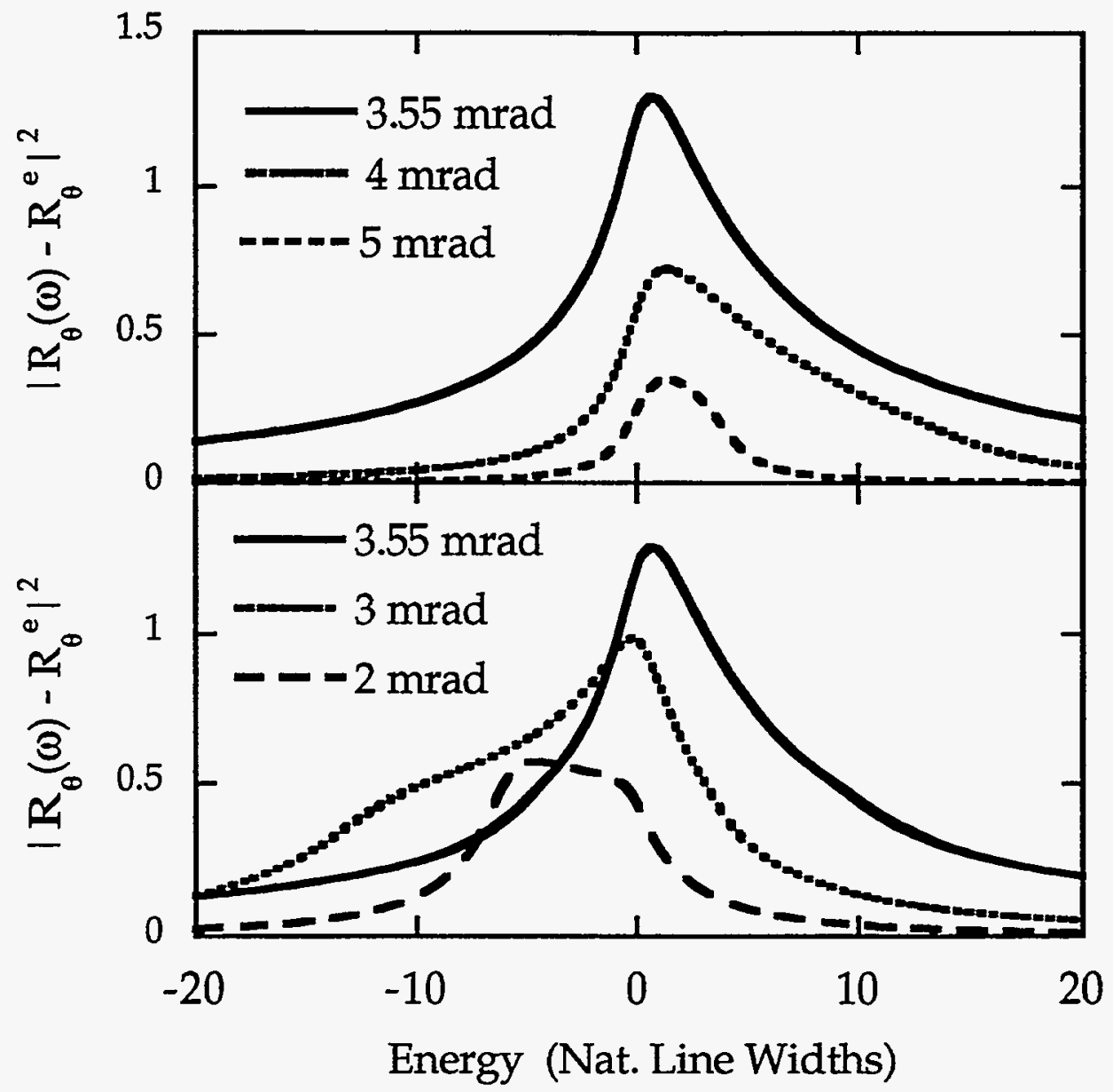

Figure 6.17. Frequency spectrum of the delayed response. See text for details. The response of the critical angle has been reproduced in both plots for comparison.

Also noticeable from figure 6.17 is that there is a shift in the centroid of the frequency response at different angles. This is plotted in figure 6.18. At large angles the frequency of the delayed radiation $(t>0)$ tends to shift above the resonance energy of an isolated nucleus, while at low angles, the shift is toward lower energies. 


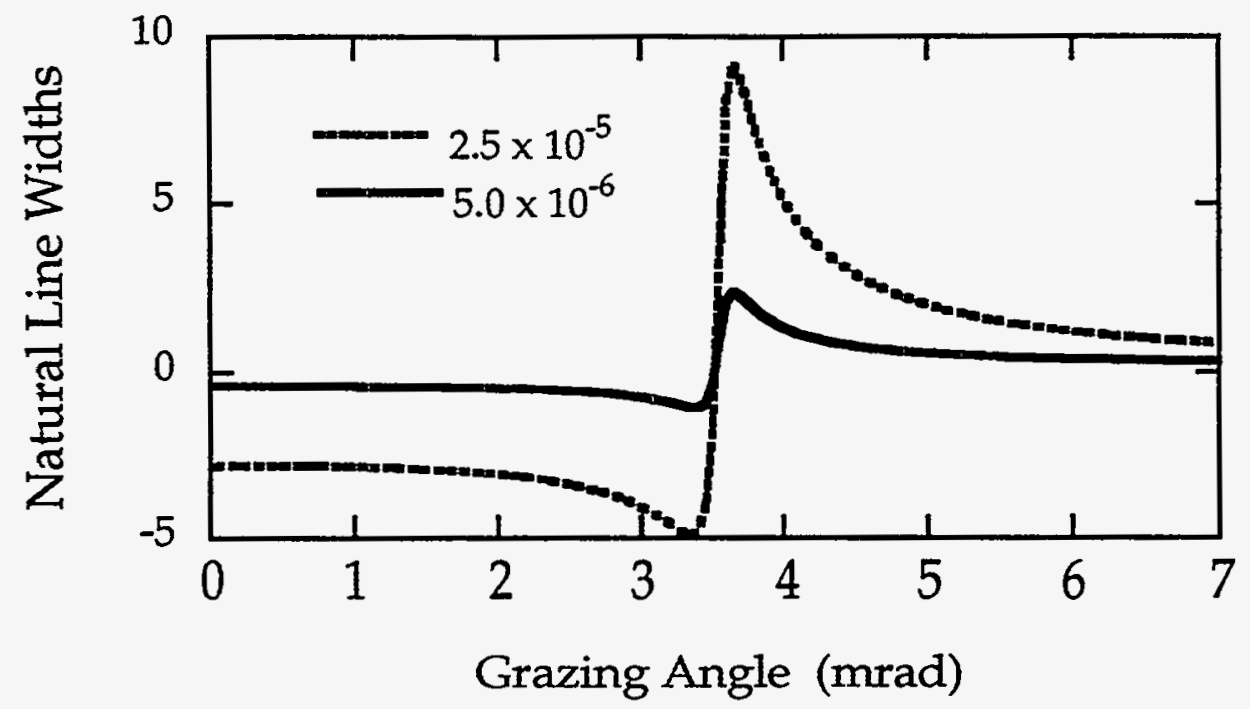

Figure 6.18. Centroid of the delayed frequency spectrum as a function of angle. The two lines are for two different values of $\delta_{n 0}$, as given. Note also that the line width of the resonance used is $2 \Gamma_{0}$.

Figure 6.19 shows the time response to an impulse excitation for each of the angles used in figure 6.16 and 6.17. Far from the critical angle, the scattering is essentially kinematic and the time response is just exponential decay with the lifetime $\hbar / \Gamma=\hbar / 2 \Gamma_{0}=\tau_{0} / 2$. In the neighborhood of the critical angle, the time response deviates from exponential decay in two ways: there is a general speed up of the response at early times and some slow modulation or beats appear, which then are no longer evident at exactly the critical angle. These effects have been described before ([Kagan, et al., 1979, 30] and [Hannon, et al., 1985, 32]). The general speed up of the response is a dynamical effect from the collective excitation of many nuclei and is just analogous to the frequency broadening observed in figure 6.17. The modulation can either be thought of as the effects of deviation of the frequency response from a simple Lorentzian, or as the beating between the collective excited state and the response of a single nucleus, which oscillate at slightly different frequencies (see [Hannon, et al., 1985, 32] and the discussion below on GIAR films). 


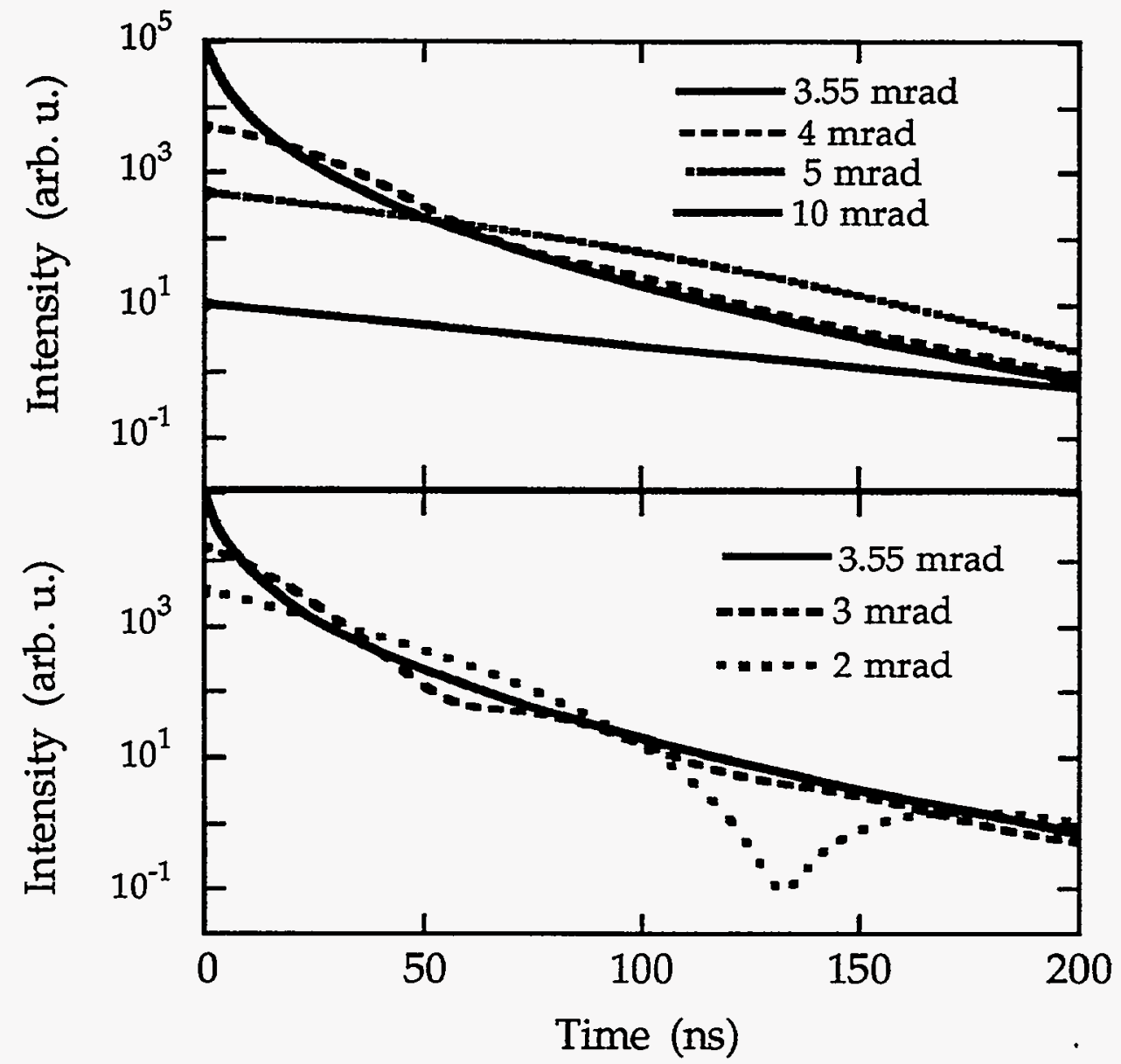

Figure 6.19. Time response at several angles in the neighborhood of the critical angle. The top plot shows angles above the critical angle while the lower plot shows angles below the critical angle. The response at $10 \mathrm{mrad}$ in the top figure shows the lifetime of free nucleus (recall the assumption of a broadened line, $\Gamma=2 \Gamma_{0}$, so this is $70 \mathrm{~ns})$.

\section{Slow Beats From the Thin Film Geometry}

The fact that the sample is a thin film results in Kiessig interference fringes in the electronic reflectivity curve (see fig. 6.1). In fact, there are also interference fringes in the frequency response. Consider the electronic reflectivity profile from a thin layer of fixed thickness $(240 \AA)$ but whose index of refraction is allowed to vary (see figure 6.20). Unsurprisingly, as $\operatorname{Re}\{\delta\}$ becomes larger, the critical angle becomes larger and the interference fringes move out to larger angles. Now consider observing the effect of the index of refraction change at 
one angle, for example the 5 mrad position shown by the vertical line in the figure. As the index increases from $\operatorname{Re}\{\delta\}=4 \times 10^{-6}$ to $\operatorname{Re}\{\delta\}=6 \times 10^{-6}$ there will be a drop in the reflected intensity. Then it will go up again as $\operatorname{Re}\{\delta\}$ approaches $8 \times 10^{-6}$ and then down again. Finally it will go up and saturate when the region of total external reflection extends out to $5 \mathrm{mrad}$.

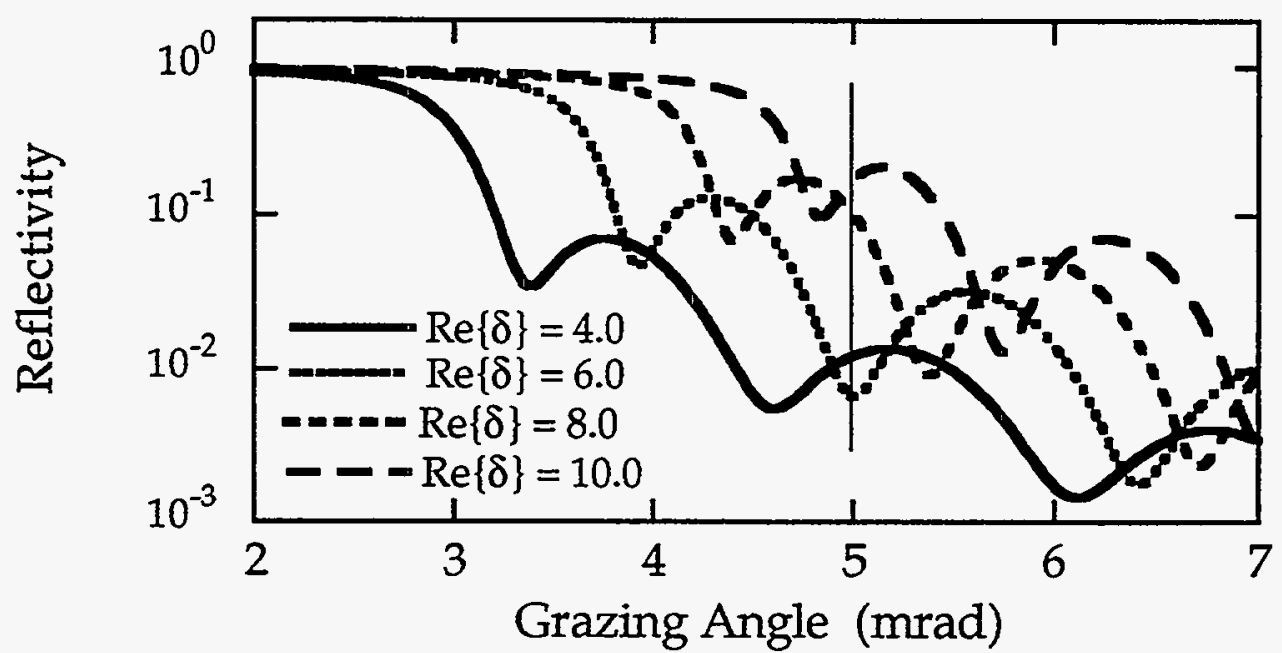

Figure 6.20. Variation in the electronic reflectivity profiles for different values of the index of refraction. $(\operatorname{Im}\{\delta\}=0.05 \operatorname{Re}\{\delta\}$ for each)

This is analogous to varying the incident frequency in the presence of nuclear scattering, and thus one expects peaks (and troughs) in the frequency response due to interference between the waves reflected from the front and back surfaces. Figure 6.20 shows the reflectivity at $5 \mathrm{mrad}$ (the same material as used for figure 6.15) in the case when there is an infinitely thick sample and for $240 \AA$ of the sample deposited on glass. The extra peak is the result of just this interference effect. The additional beating in the time response is also clear. 

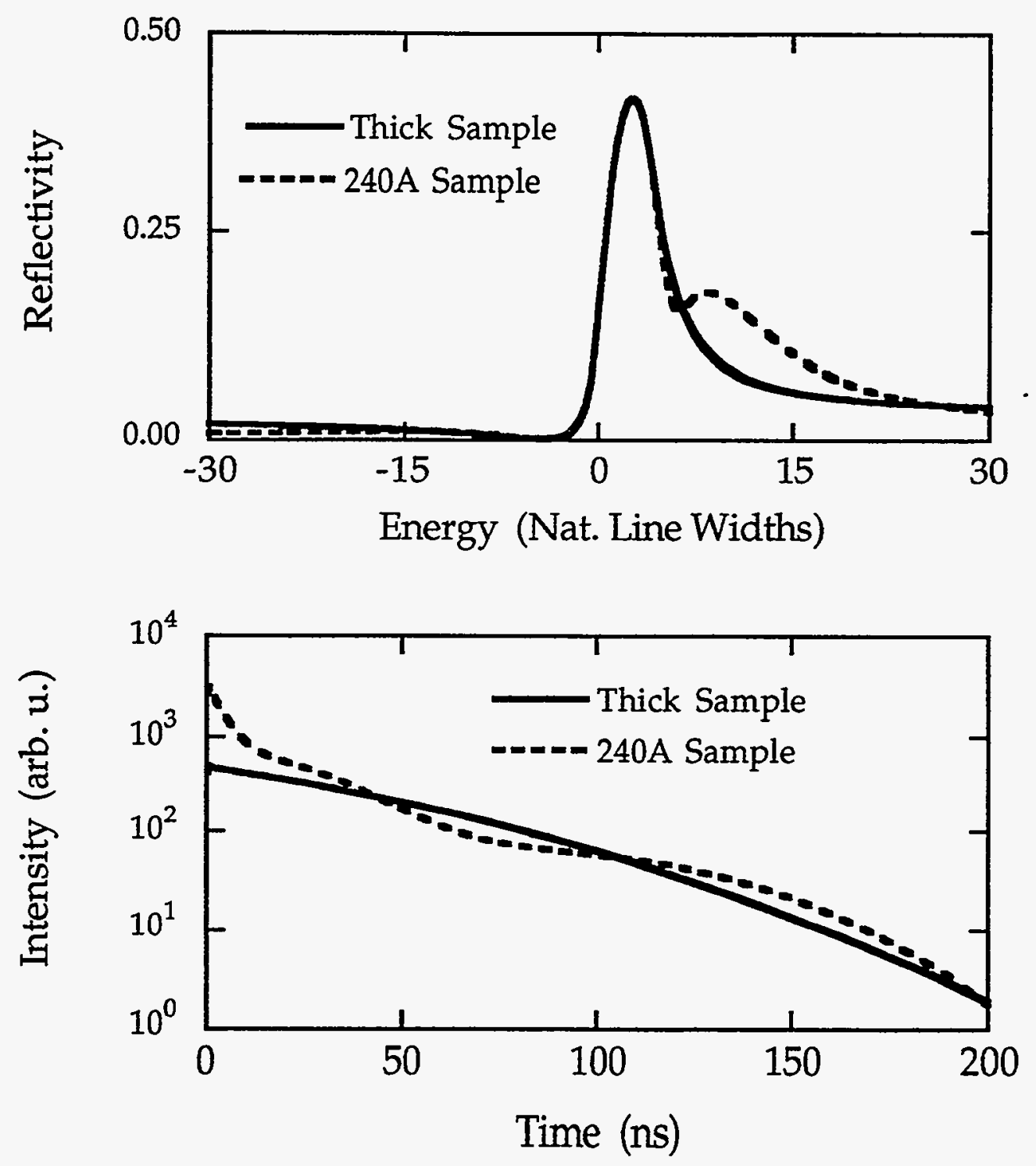

Figure 6.21. Comparison of the time and frequency responses of a thick (infinite) sample and a thin layer on glass. Index of refraction profile as given in figure 6.15

It is worth emphasizing that while Kiessig beats in electronic reflectivity profiles are essentially a kinematic effect of the geometry, this interference effect is essentially a dynamical one. It is analogous to the shift in the Kiessig pattern at different values of the index, which results from the refraction of the wave inside the material. 


\section{Dynamical Effects on the Quantum Beat Frequency}

In addition to creating slow beats in the time response, dynamical scattering can also influence the faster (kinematically based) quantum beats in two different ways. On the one hand, there is the dynamical shift that occurs for a single line discussed above (e.g. fig 6.18). If two lines of different strengths are excited by the same incident wave (and the same polarization component of that wave) then the stronger line will be shifted more than the weaker one and there will be a change in the frequency difference between them.

Another effect is that, when two (or more) resonances are excited by the same incident polarization, the tails of one resonance can modify the "location" of another resonance. Thus, for example, the response in the ll cases is primarily a two line response (for each eigenpolarization) where the amplitude of one line is three times that of the other in a kinematic limit (see figure 6.11). However, at grazing incidence, the response is no longer linear in the scattering amplitude so that a small addition to the amplitude can have a large effect (e.g. a shift) on the resonance. Thus the small line will be shifted by the presence of the large line, and vice-versa, though not as much.

The effect of these shifts is illustrated in figure 6.22 for a two line case similar to the ll scattering geometry used above: two lines separated by about $63 \Gamma_{0}$ with relative strengths $1: 3$. In the kinematic limit, these lines are at the locations $\pm 31.5 \Gamma_{0}$. However, at $5 \mathrm{mrad}$ grazing incidence, the locations are approximately $\left(-30.1 \Gamma_{0}, 32.1 \Gamma_{0}\right)$ for the pair (large, small) and $\left(-31.0 \Gamma_{0}, 33.0 \Gamma_{0}\right)$ for the other pair. The dynamical shift for single lines tends to move all of the lines to higher energies (though large lines more than small lines) while the effects of the tails of one line shifts one set further apart than the other. The net effect is a shift of about $2 \Gamma_{0}$, or $3 \%$, in the relative splittings in the two cases. The beat periods in the time response should also be shifted. 


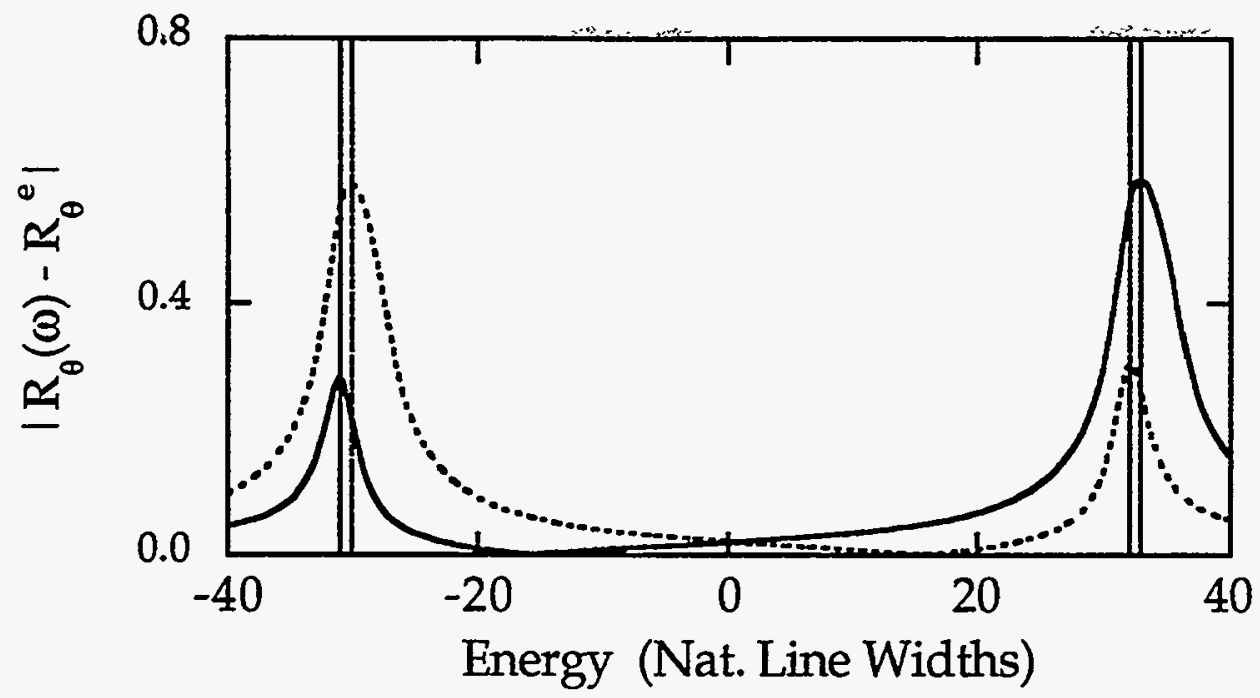

Figure 6.22. Effect of dynamical scattering on the line separations. Note the vertical scale is an amplitude.

The time response at several angles is shown in figure 6.23. The shift in beat periods for the two different cases is evident. For the $5 \mathrm{mrad}$ case roughly 13 periods are necessary to shift out of phase by 180 degrees, in reasonable agreement with the approximately $3 \%$ shift in period expected from figure 6.22. The effect becomes more pronounced at smaller angles, though the full line shape also has some influence. For the largest angle shown, $20 \mathrm{mrad}$, the response is essentially kinematic, and the beat pattern is independent of the order of the lines. 


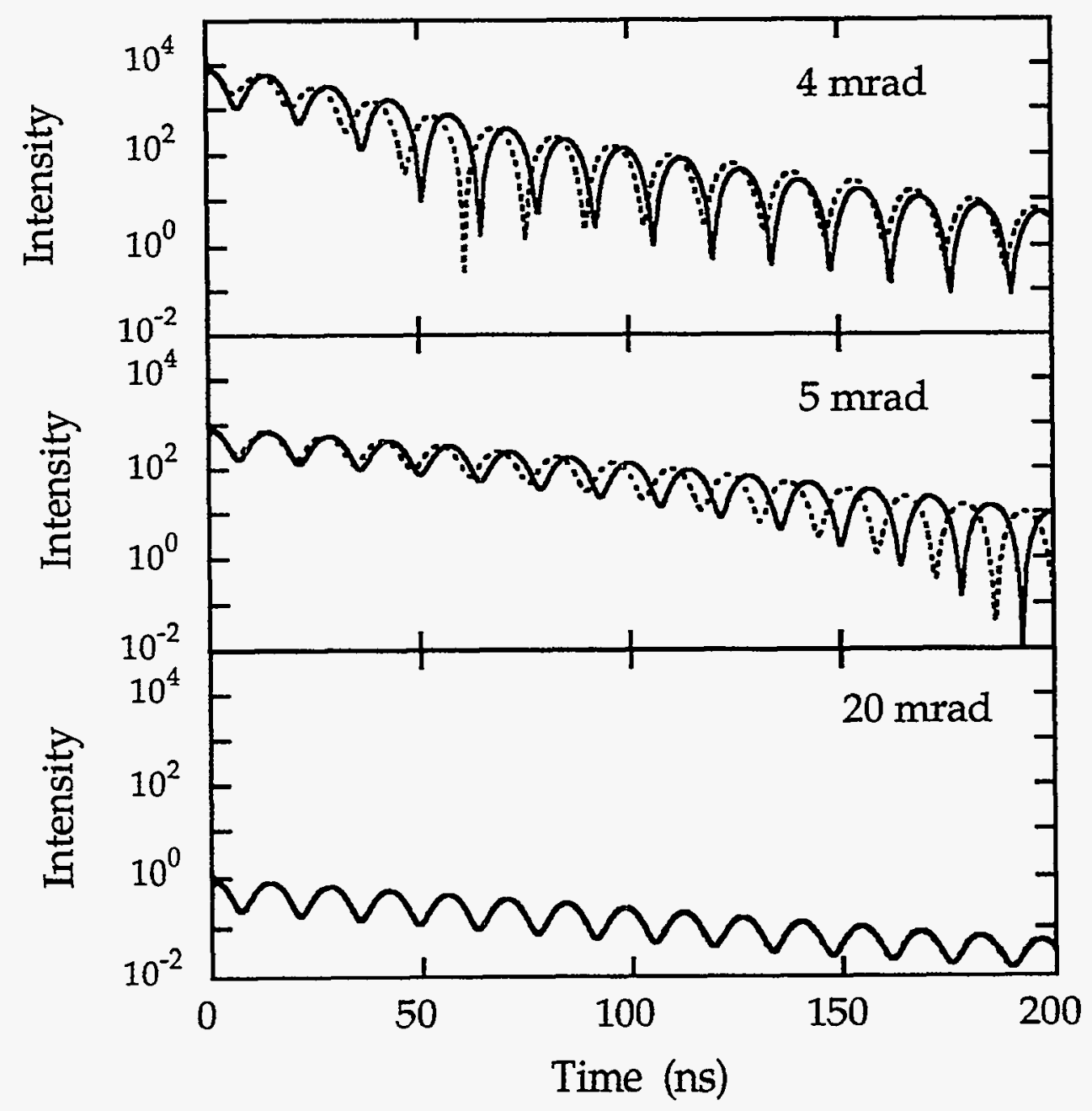

Figure 6.23. Effect of dynamical shifts on the time response. See text for details.

\section{Dynamical Modification of the Contrast}

In comparing the kinematic time response with the high angle (113) response (see figure 6.12) it was noted that the contrast of the data was larger than that of the kinematic calculation. The same effect is apparent in figure 6.21, where the response at small angles shows better contrast than the large angle (20 mrad) response. This results from the non-linear dependence of the reflected amplitude on the scattering amplitude or $\delta$. The strong lines tend to saturate more quickly than the weak ones (as the critical angle is approached) so that the 
saturation tends to equalize the strength of the lines, which improves the contrast of the beats. This is evident in figure 6.22, where the kinematic amplitude ratio of the two lines used in the calculation is 3:1, while that in the (dynamically) reflected beam is closer to 2:1. However, one notes that this improved contrast is partially canceled out in the ll case by the beat period shift noted immediately above which tends to blur the minima somewhat. In the perpendicular case, the addition of more lines, and the fact that they are weaker, reduces the visibility of this effect.

\section{Comparison with GIAR films}

We take a moment here to compare simple specular reflection to reflection from GIAR films. With some small changes, many of the same effects are present, and the GIAR case has been studied in some detail by Hannon et al. [Hannon, et al., 1985, 3, Hannon, et al., 1985, 32]. In particular, there is an analytic form for the time response for an impedance matched quarter wave GIAR film. In this structure, a thin layer of (non-resonant) material has been deposited on top of a substrate with a resonant transition so that the electronic reflectivity (i.e. far from resonance) is small. Of course, this is only true at one angle for any given sample.

The equations of chapter 4 may be used to construct the reflectivity of a simple two layer system (isotropic layers assumed). One has

$$
R_{\mathrm{GIAR}}=\frac{\mathrm{R}_{1}+\mathrm{e}^{\mathrm{i} \varphi} \mathrm{R}_{2}}{1+\mathrm{e}^{\mathrm{i} \varphi} \mathrm{R}_{1} \mathrm{R}_{2}}
$$

where $R_{1}$ and $R_{2}$ are the Fresnel reflectivity of the top and lower interface, respectively, and $\varphi$ is a phase factor from passing through the top layer. Assuming the bottom layer material has a resonant transition, one writes the reflectivity as

$$
R_{2}=\frac{1-\beta_{2}}{1+\beta_{2}}=\frac{1-\beta_{n} \beta_{e}}{1+\beta_{n} \beta_{e}}
$$


where the reflectivity of the lower layer far from resonance is just $\left(1-\beta_{\mathrm{e}}\right) /\left(1+\beta_{\mathrm{e}}\right)$. Explicitly one has

$$
\begin{aligned}
& \beta_{e}=\left[\frac{\theta^{2}-2 \delta_{2 e}}{\theta^{2}-2 \delta_{1 e}}\right]^{1 / 2} \\
& \beta_{n}=\left[1-\frac{2 \delta_{2 n}(\omega)}{\theta^{2}-2 \delta_{2 e}}\right]^{1 / 2}
\end{aligned}
$$

where the subscripts 1 and 2 refer to the top and bottom (substrate) layers, and the top layer has been assumed to be non-resonant $\left(\delta_{2 n}=0\right)$. The condition that the reflectivity of the pair of layers be zero far from resonance then allows one to express $R_{1}$ in terms of $\beta_{e}$ giving

$$
\mathrm{R}_{\mathrm{GIAR}}=\frac{1-\beta_{\mathrm{n}}}{1+\beta_{\mathrm{n}}} \mathrm{e}^{\mathrm{i} \varphi}
$$

This is the grazing incidence analog to a pure nuclear Bragg reflection, where the electronic forward scattering is included, but there is no electronic contribution to the reflected wave. Ignoring the phase factor, the effect of the electronic forward scattering is just to shift the effective angle of incidence onto the material, essentially providing refraction of the wave, without scattering.

The time response (Fourier transform of (6.12)) may be determined analytically. This was done by Hannon, et al ., [Hannon, et al., 1985, 32] giving

$$
G_{\theta}(t)=-i e^{-i\left(\omega_{0}+\omega_{B}\right) t} e^{-\Gamma t / 2 \hbar} \frac{J_{1}\left(\omega_{B} t\right)}{t}
$$

Where $\omega_{\mathrm{B}}$ is a complex beat frequency given by ${ }^{*}$

$$
\omega_{\mathrm{B}}=\frac{\delta_{\mathrm{n} 0} \Gamma / 2 \hbar}{\theta^{2}-2 \delta_{\mathrm{e}}}
$$

\footnotetext{
${ }^{*}$ For comparison with Hannon, et al, $\delta_{\mathrm{n} 0}=\mathrm{f}_{0} \lambda^{2} \mathrm{~N} / 2 \pi$.
} 


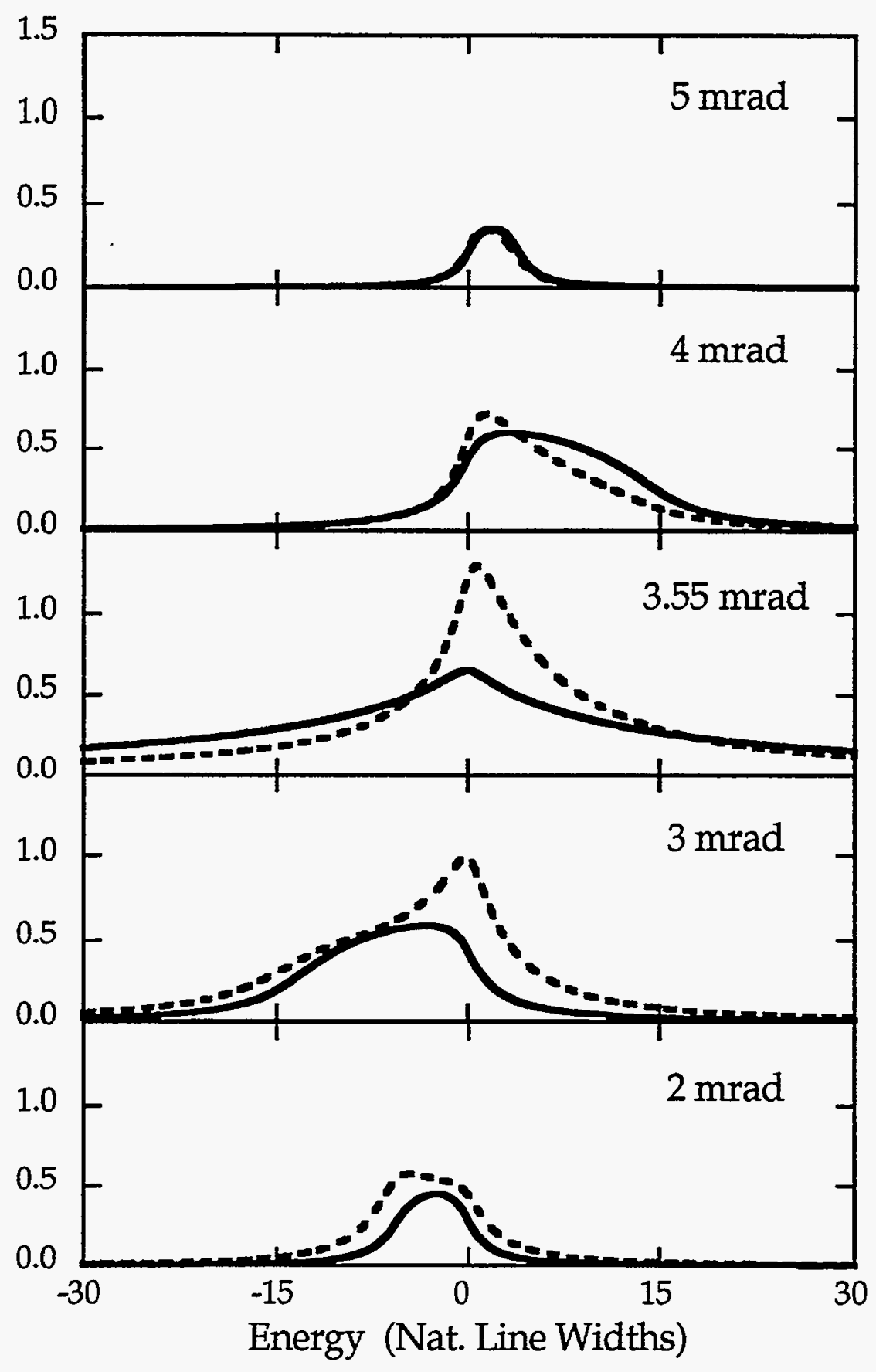

Figure 6.24. Frequency response for a material with a single line transition in the GLAR approximation (solid line) and for specular reflection (dashed line).

For intermediate time scales, $1 / \operatorname{Re}\left\{\omega_{\mathrm{B}}\right\}<\mathrm{t}<1 / \operatorname{Im}\left\{\omega_{\mathrm{B}}\right\}$ the Bessel function is approximately a cosine (with an offset) so $\omega_{\mathrm{B}}$ corresponds to a beat frequency. This is visible in both the frequency response and the time response, as shown in 
Figures 6.24 and 6.25. Hannon, et al, suggest the beat frequency is due to competition between the response of a single nucleus at the resonance energy and an angle dependent response of the collective excitation.

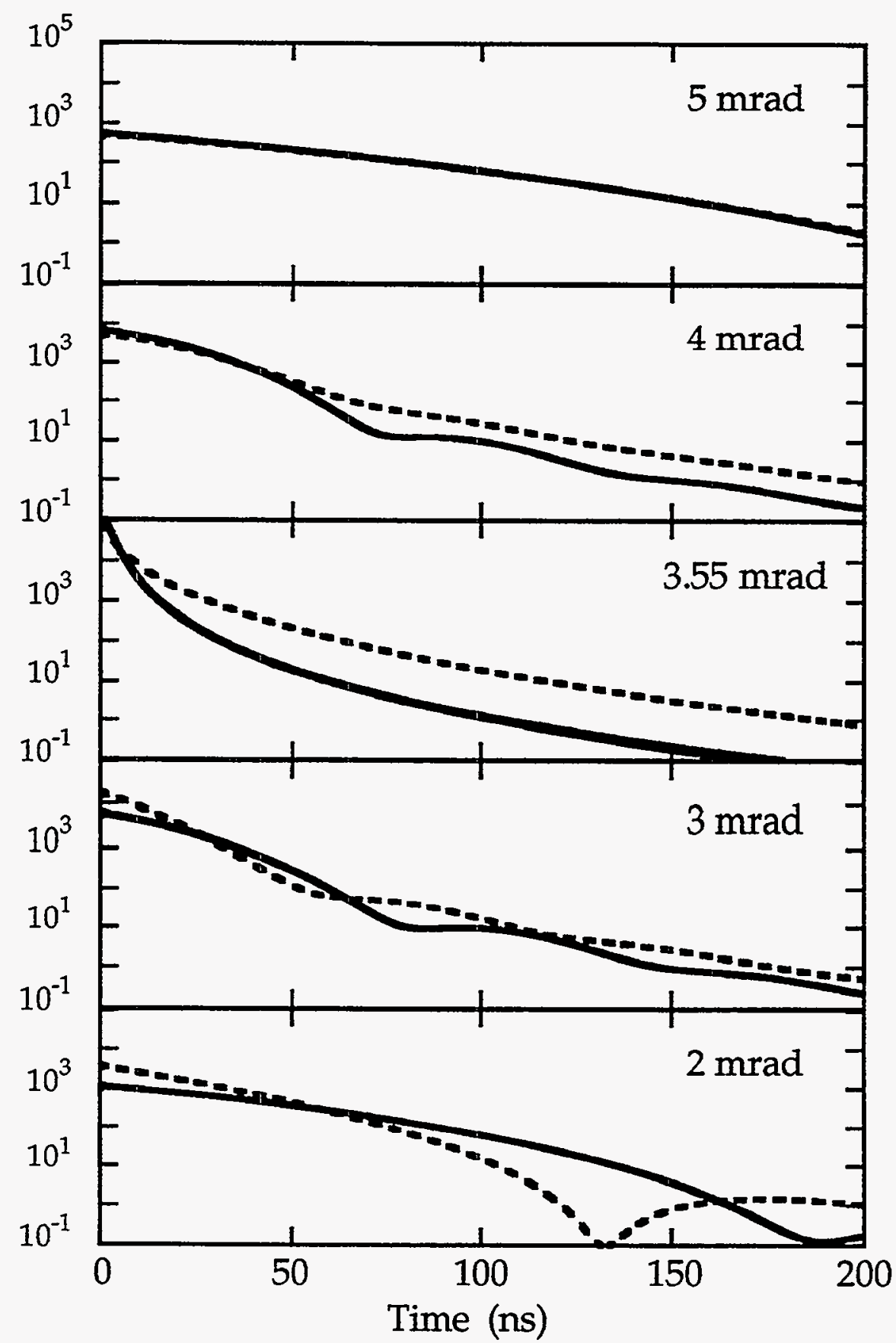

Figure 6.25. Time response in GIAR (solid line) limit and in the case of specular reflection (dashed line). 
It is also worth noting that in the event there is no electronic absorption (i.e. $\delta_{e}$ is real) then the beat frequency is purely real and the minima in the GIAR time response will become zeros. However, for the specular reflection case, simulations show this is not the case. Even if there is no electronic absorption, the minima will be blurred.

\section{Fitting the Time Response}

The time response of the iron layer was fit using the theory of chapters 4 and 5 with the three layer model of the electronic structure described above. The fitting code was a non-linear fit to chi squared based around the Numerical Algorithms Group (NAG) FORTRAN library routines E04UCF and E04XAF [Numerical Algorithms Group, 33]. Initially the time response for each angle and magnetic field orientation was fit independently. However, the fact that different fits gave different values for parameters that should not have changed (e.g. the structure of the layer should not change from one angle to another) finally led to fitting all of the spectra simultaneously. For each time response measurement, the code was allowed to independently vary the normalization, $t=0$ position, and angle within reasonable limits about the expected values. However, each time a structural parameter that should affect the response at every angle was changed, all of them were recalculated. The fitting was slow, but finally gave consistent results. The chi squared used was the sum of the chi squareds from each spectrum.

Two other parameters that were allowed to vary were the fraction of $\mathrm{pi}$ polarized component in the incident synchrotron radiation beam and a scale factor to account for possible miscalibration of the time to amplitude converter or of the velocity scale for the CEMS measurement. The former was allowed to vary between 0 and $10 \%$, and was typically about $5 \%$ in the better fits, in reasonable agreement with expectations given the beamline and the optics. The latter was allowed to vary between 0.99 and 1.01 and was typically $\sim 0.997$, certainly within experimental error.

Many different models were used in the fitting procedure, but the essential results are described by the four models presented in table 6.2. The first 
fit simply assumed that the iron in the sample was entirely homogeneous in its nuclear response: the only free parameters were those listed above for each time spectrum, and the relative amounts of the broad and narrow components from the fit to the CEMS. The sample was assumed to be entirely iron with the density profile given by the three layer model of the electronic structure described above. In fact the resonant fraction* of the layer was allowed to vary below the $77 \pm 2 \%$ expected for bulk iron, but practically, the fitting procedure invariably chose the maximum value (up to about $85 \%$ ).

$\begin{array}{llll}\text { Fit } 1 & \text { Fit } 2 & \text { Fit } 3 & \text { Fit } 4\end{array}$

$\begin{array}{ccccc}\text { Res. Fract. } & & & & \\ \text { Total } & 0.79 & 0.70 & 0.79 & 0.75 \\ \text { Layer 1 } & & 0.00 & " & 0.73 \\ \text { Layer 2 } & & 0.79 & " & 0.79 \\ \text { Layer 3 } & & 0.56 & & 0.56 \\ & & & & \\ \text { Brd. Comp. } & & & & \\ \text { Total } & 0.42 & 0.35 & 0.36 & 0.38 \\ \text { Layer 1 } & " & n & 1.00 & 1.00 \\ \text { Layer 2 } & " & n & .36 & 0.36 \\ \text { Layer 3 } & & n & .10 & 0.10 \\ & & & & \\ \text { Pi Poln. } & 0.054 & 0.064 & 0.031 & 0.032 \\ & & & & \\ \chi^{2} / \text { pt } & 17.2 & 12.8 & 11.0 & 10.2 \\ & & & & \\ 110 & 8.8 & 7.3 & 7.8 & 8.0 \\ \text { I11 } & 5.3 & 3.4 & 7.2 & 7.0 \\ \text { 112 } & 14.4 & 8.7 & 12.7 & 10.3 \\ 113 & 8.6 & 5.0 & 3.6 & 2.8 \\ & & & & \\ \text { pp1 } & 38.7 & 32.2 & 21.2 & 19.7 \\ \text { pp2 } & 31.1 & 22.2 & 17.8 & 16.2 \\ \text { pp3 } & 7.9 & 5.2 & 4.1 & 5.1\end{array}$

Table 6.2. Results from fitting the time response measured from the iron layer.

\footnotetext{
* The correction for the $95 \%$ enrichment for the sample is included.
} 
The other fits varied the nuclear response in the same 3 layer divisions found from fitting the electronic response. These layers were (see the early part of the chapter) : a top layer of $24.7 \AA$ and 0.51 bulk density, a central layer of $174.0 \AA$ and 0.86 bulk density and a back layer of $36.6 \AA$ and 0.88 bulk density, with $3.7 \AA$ roughness at each interface. The fits independently varied the resonant fraction of each layer (fit 2), the fraction of the broad component of the iron in each layer (fit 3 ) or both (fit 4 ). The results from fit 4 are plotted in figures 6.26-6.29, on both linear and logarithmic scales.

The $\chi^{2}$ /point values in table 6.2 are all rather above unity, suggesting that there are systematic errors in the model used to fit the data. This is not surprising: a three layer model is a vast over-simplification of the problem. In addition, one notes that the $\chi^{2}$ values for the fits to $\mathrm{pp} 1$ and $\mathrm{pp} 2$ are notably high, relative to the others. This is due the fact that the data quality is much better for these cases: the spectra were taken for longer periods of time (and the time response decays more slowly in the pp than the 11 cases) so there is more $\mathrm{data} /$ channel, and hence, the error bars, which are statistical (counts ${ }^{1 / 2}$ ), are fractionally smaller. A higher $\chi^{2}$ is exactly what one would expect with systematic errors. Nonetheless the fits appear quite good on both linear and log scales.

The fits to 113 and pp3 tend to have low $\chi^{2}$ values, relative to the other fits. This is partially the result of the fact that the count rates tended to be lower for these measurements. In addition, however, it is probably due to the fact that these measurements are more nearly in a kinematic scattering limit. As has been pointed out in several places in this thesis, in a kinematic limit the shpe of the time response is not very sensitive to geometry and becomes much simpler: all of the dynamical modifications described in the early part of this chapter do not take place. While these responses are certainly not kinematic (see figures 6.12 and 6.14) they are probably less effected by the details of the structure than the responses measured closer to the critical angle. 


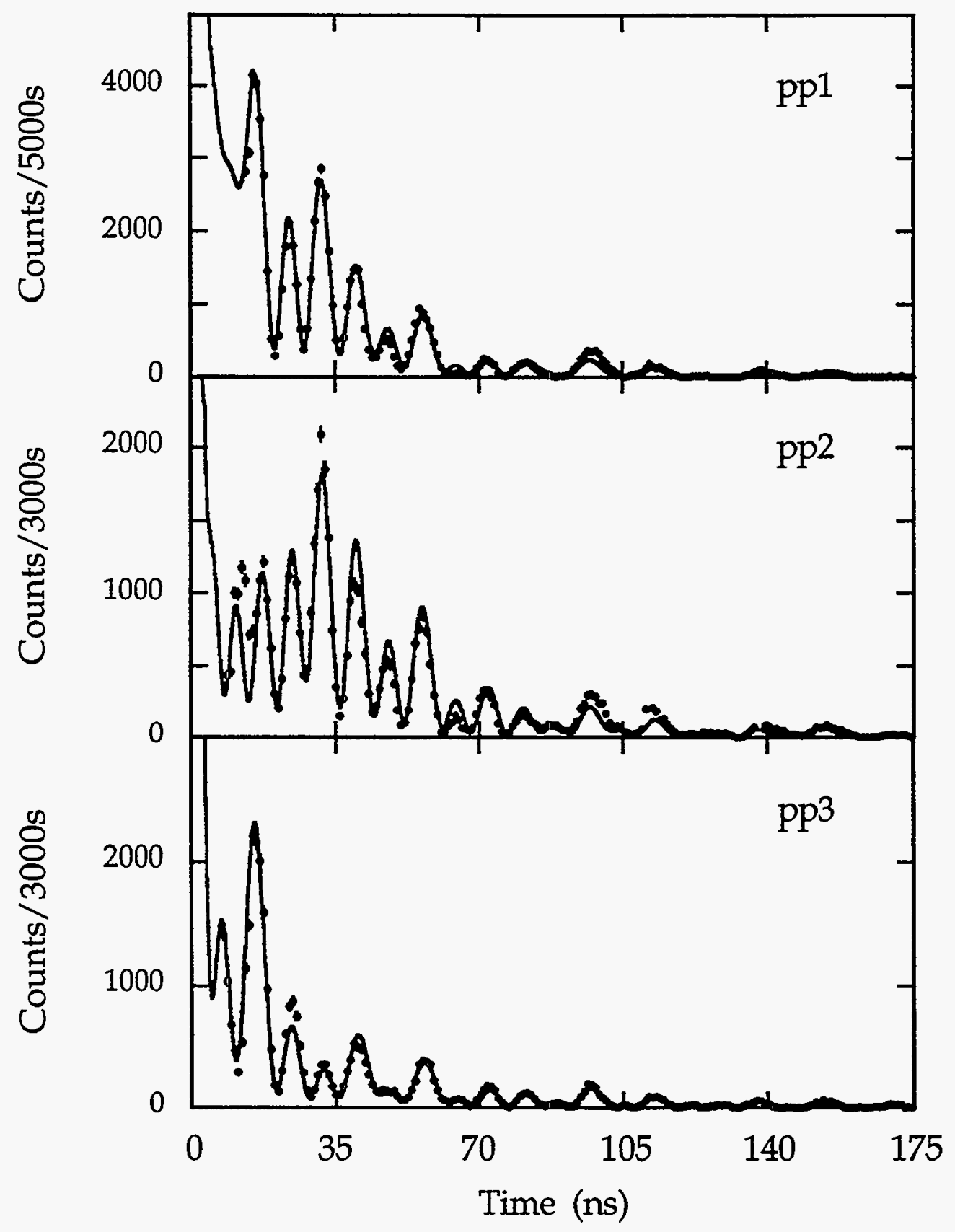

Figure 6.26. Measured time response from the iron layer at several angles with the magnetic field perpendicular to the scattering plane. Linear scale. The solid line is from fit 4 , as described in the text and table 6.2 


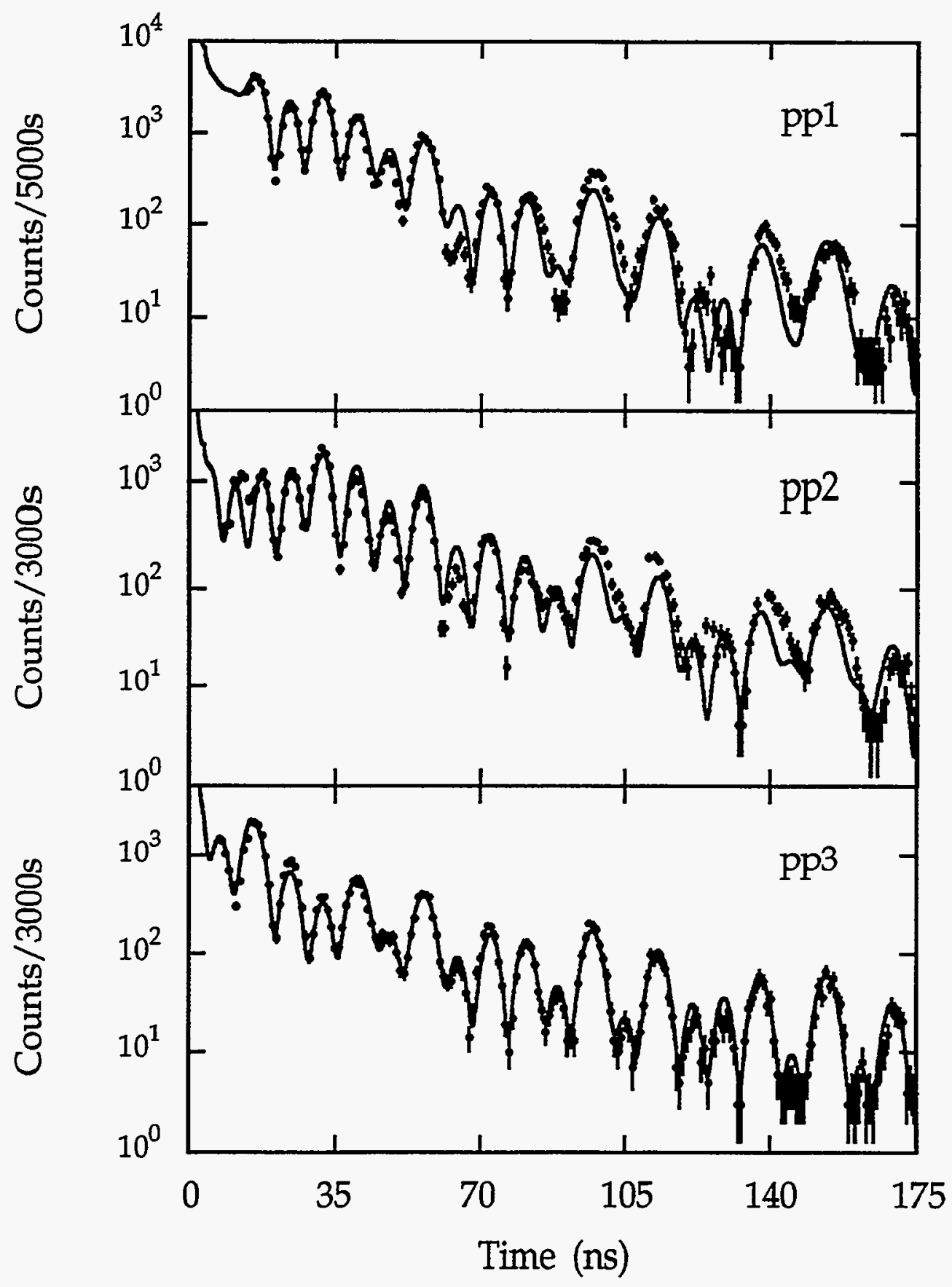

Figure 6.27. Measured time response from the iron layer at several angles with the magnetic field perpendicular to the scattering plane. Logarithmic scale. The solid line is from fit 4 , as described in the text and table 6.2 


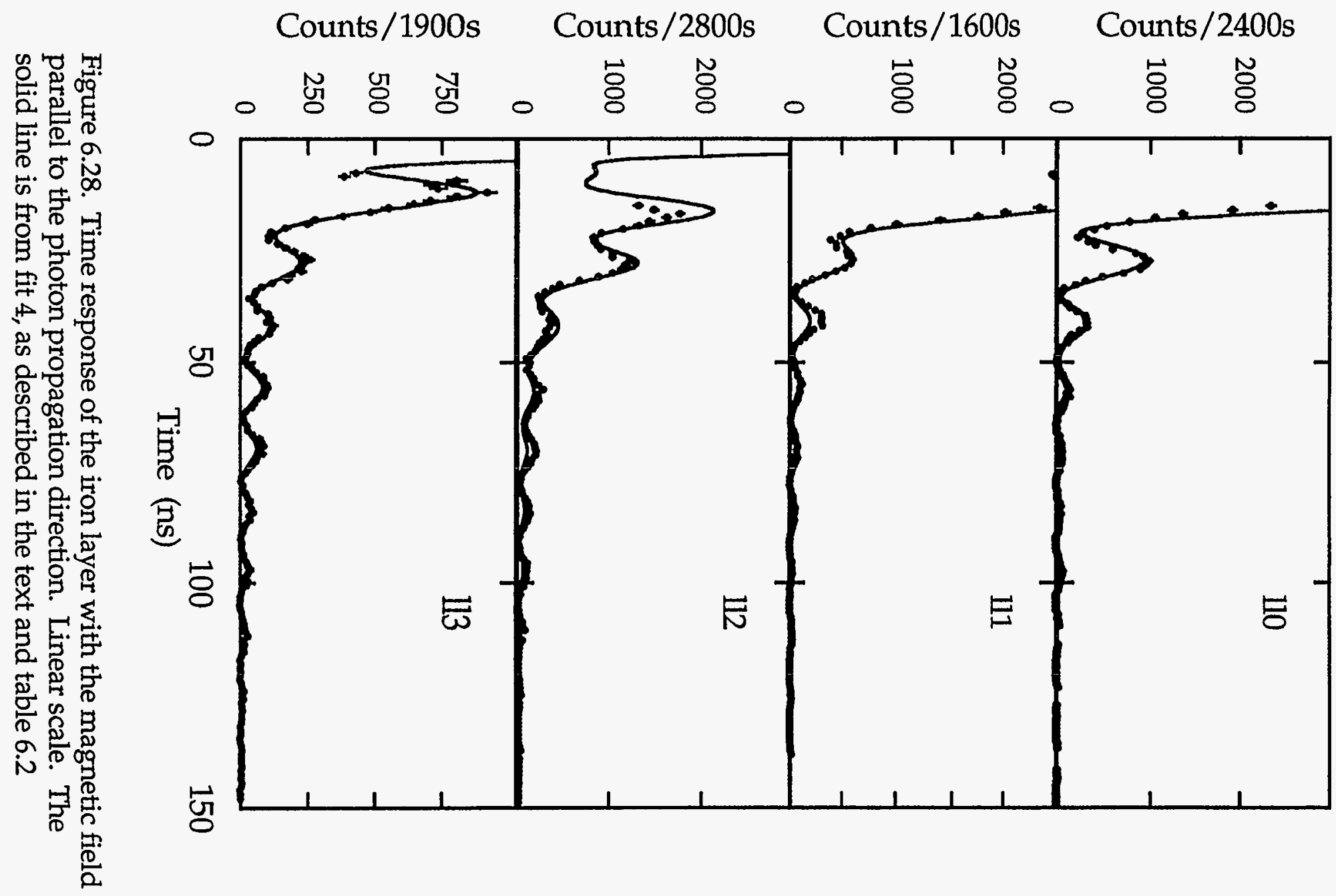




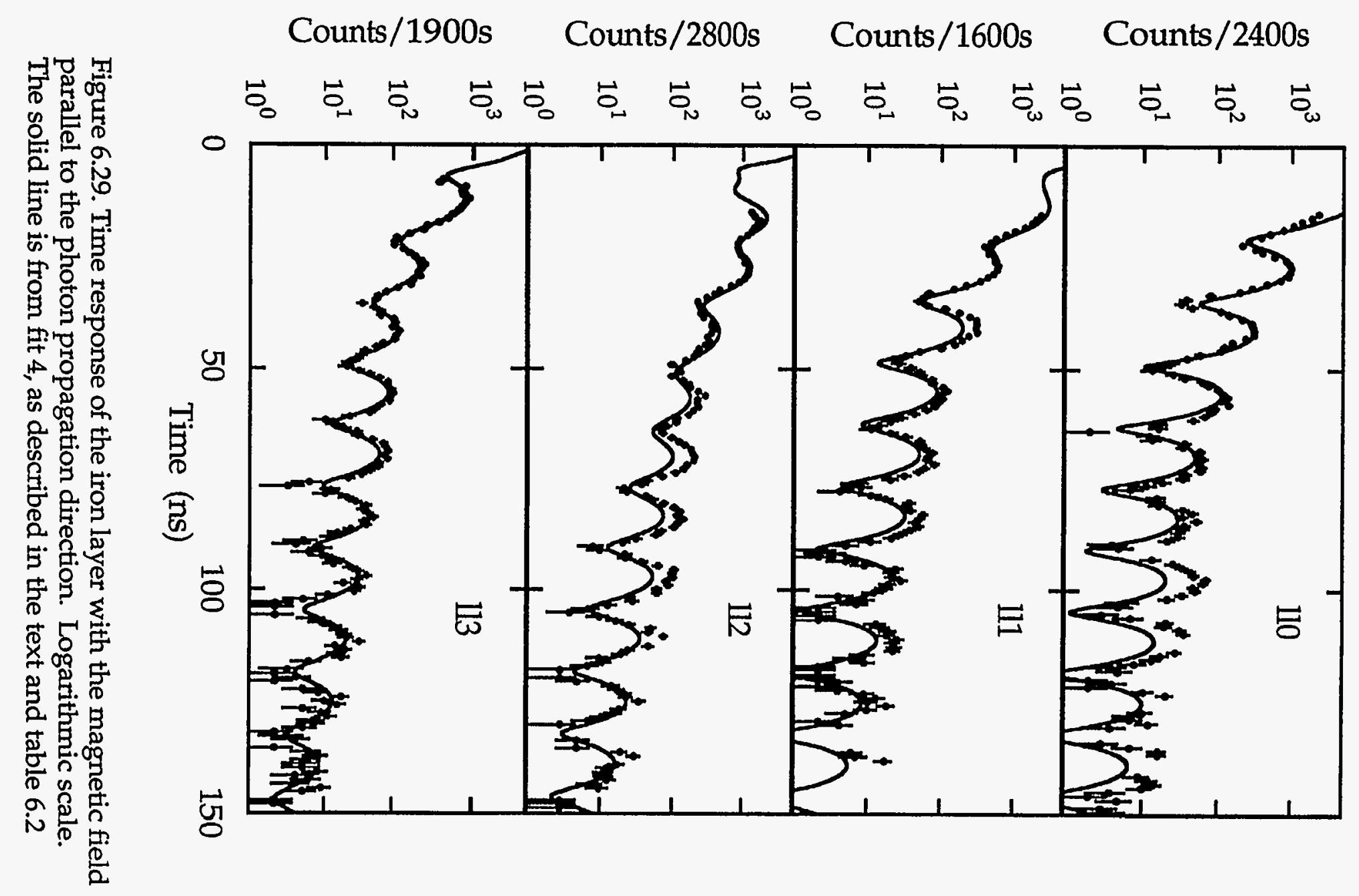


Given large values of $\chi^{2}$ one reasonable question is what can be done to improve the model. Thus various modifications in the structure were attempted. Finally, the fits above were the best obtained, but it is certainly worth describing what has been tried.

The first attempt at improvement was to include a small amount of oxide on the surface (see the discussion about conversion electron spectra). Thus a new sextet of lines was included in the top layer, at positions appropriate for the magnetite. The code was allowed to vary the amplitude, the width and the locations of these lines, but it lead to no significant improvement in $\chi^{2}$. Likewise a doublet, corresponding to the $\mathrm{Fe}^{3+}$ oxidation state observed by some authors[Brundle, 1978, 34] [Frost, et al., 1985, 35] [Stadnik, et al., 1989, 15] [Irkaev, et al., 1993, 36] was included in the top layer, again without improvement.

The work by Isaenko, et al [Isaenko, et al., 1994, 9l, suggested that in a field of 200 oersted, there was some distribution of quantization axis directions (i.e. not all magnetic domains were exactly aligned with the field). Thus, although the field was higher in this work ( $\sim 300$ oersted), fits were attempted including some variation in the domain directions. This did not improve the fits. Also, the code was allowed to include some un-oriented iron in each layer (possibly due to the finite size of domain walls) again without improvement.

Thus, it was not possible to significantly improve on the fit, 4, presented above. The reason is very probably the use of the approximate three layer structure to describe what is really a continuously varying distribution. However, after gaining substantial experience fitting the electronic reflectivity profile, it became clear that it was not productive to spend excessive amounts of time fitting the nuclear response with a many-layer profile. The number of free parameters becomes very large and there is no way that this author knows of ascertaining the correctness (uniqueness) of the final result.

The fits of the time response allow two conclusions. The basic features of the response are very well understood and their variation with angle is consistent with this model. There is also very good evidence that the top portion of the sample is largely composed of iron having a line width that is broader than the bulk. There are also some ideas that occur about how similar investigations may 
be improved in the future. This is discussed in more detail below. Now we discuss one more measurement with iron layer.

\section{The Integrated Delayed Reflectivity}

It is interesting to investigate the integrated delayed counting rate of the reflected $\mathrm{x}$-rays from the thin iron layer as a function of angle. In some sense, this is the nuclear analog of the electronic reflectivity profile shown in figure 6.1. Of course, using the theory described in chapter 4 , this is simple to calculate. However, the results of such calculations seemed at variance with some of the expectations of other workers in the field, based on their experience with pure nuclear Bragg reflections. Thus we measured this response in a subsequent experiment at SSRL.

The quantity that was measured is proportional to what will be called the "integrated delayed intensity" or just the "delayed intensity". It is related to the time response by

$$
I_{\theta}=\int_{0^{+}}^{+\infty}\left|G_{\theta}(t)\right|^{2} d t
$$

The $0^{+}$lower bound on the integral is meant to exclude the prompt pulse due to electronic scattering. Experimentally, due to the detector response, this was about $4 \mathrm{~ns}$. Application of Parseval's theorem, and noting that $\mathrm{G}(\mathrm{t})$ is causal $(\mathrm{G}(\mathrm{t}<0)=0)$ gives

$$
I_{\theta}=\frac{1}{2 \pi} \int_{-\infty}^{+\infty}\left|R_{\theta}(\omega)-R_{\theta}^{e}\right|^{2} d \omega
$$

This directly relates the delayed intensity to what was previously called the "delayed frequency spectrum" plotted in figure 6.17.

In a weak scattering (kinematic) limit, e.g. $\theta>\theta_{c}, R_{\theta}(\omega)$ separates into the sum of two terms. one due to electronic scattering and one due to nuclear scattering. The electronic term cancels out of (6.16) and the delayed intensity is 
strictly a function of nuclear parameters. However, in the vicinity of the critical angle, the scattering is very much dynamical and no such separation is possible. The numerical result is shown in figure 6.30 for the simple case of the reflection from a semi-infinite sample of the material used in the simulations above (e.g. figure 6.15). Also shown is the integrated reflectivity for a pure nuclear reflection in the GIAR film case, which will be discussed in the next section.

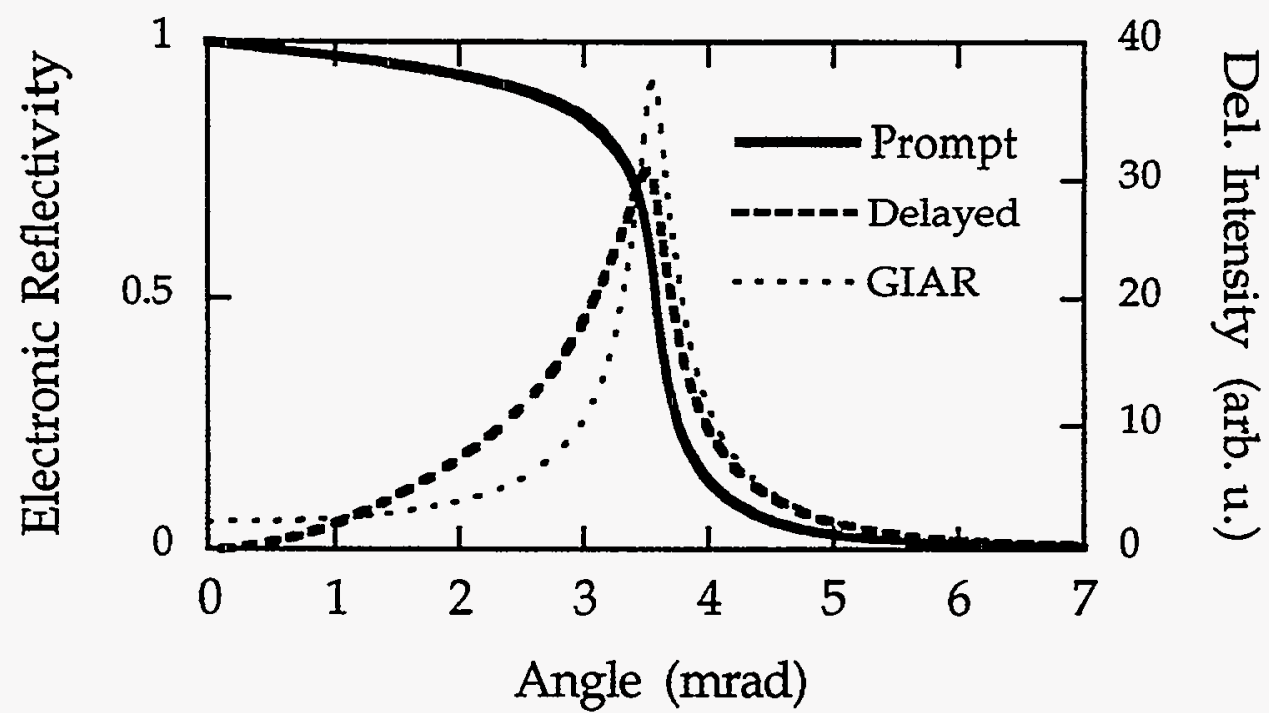

Figure 6.30. Electronic reflectivity and delayed intensity as a function of grazing angle. Note that the "arb. u." scale in this case can be interpreted as the number of delayed photons that should reflected for an incident flux of one photon per natural line width.

The delayed intensity is clearly seen to peak at the electronic critical angle. This can be explained by noting that the delayed intensity will be high when the magnitude of the difference $\left|R_{\theta}(\omega)-R_{\theta}^{e}\right|$ is large over an appreciable frequency range. Since $R_{\theta}(\omega)$ depends on frequency only through $\delta_{n}(\omega) / \theta^{2}$, one would expect the difference to be most sensitive to changes in frequency at angles where $R_{\theta}^{e}$ is most sensitive to changes in angle. More formally, expansion to first order in $\delta_{n}(\omega) / \delta_{\mathrm{e}}$ (the validity of this expansion will be discussed below) gives

$$
R_{\theta}(\omega)-R_{\theta}^{e} \approx-\frac{\theta}{2 \delta_{e}} \frac{d R_{\theta}^{e}}{d \theta} \delta_{n}(\omega)
$$


This explicitly shows the dependence of the delayed intensity on the derivative of the electronic reflectivity with angle. The separation of the frequency and the angular dependence means it is reasonable to think of the integral in equation 6.17 , the delayed intensity, as being large where there is a fast change in the electronic reflectivity with angle (i.e. at the critical angle).

Evaluation of the derivative in equation 6.17 gives

$$
\begin{aligned}
\mathrm{R}_{\theta}(\omega)-\mathrm{R}_{\theta}^{\mathrm{e}} & \approx \frac{2}{1+\beta_{\mathrm{e}}} \frac{2 \beta_{\mathrm{e}}}{1+\beta_{\mathrm{e}}} \frac{\delta_{\mathrm{n}}(\omega)}{2 \theta^{2} \beta_{\mathrm{e}}^{2}} \\
& =\mathrm{T}_{\theta}^{\mathrm{e}} \mathrm{T}_{\theta}^{\mathrm{e}} \tilde{\mathrm{R}}_{\theta}(\omega)
\end{aligned}
$$

where $T_{\theta}^{e}=2 /\left(1+\beta_{e}\right)$ is the (Fresnel) amplitude transmission coefficient into the electronic material (without nuclei present) and $T_{\theta}^{\prime e}=2 \beta_{e} /\left(1+\beta_{e}\right)$ is the transmission back out. $\tilde{R}=\delta_{n} / 2 \theta^{2} \beta_{e}{ }^{2}$ is the reflection coefficient for an interface between the electronic material, index $1-\delta_{\mathrm{e}}$ and a material with index of refraction $1-\delta_{e}-\delta_{n}(\omega) . \tilde{R}$ has the characteristic $1 / q^{2}$ amplitude ( $1 / q^{4}$ intensity) dependence of kinematic small angle scattering, where $q$ is the momentum transfer in the material $\left(q \propto \theta \beta_{e}=\left[\theta^{2}-2 \delta\right]^{1 / 2}\right)$. This quickly reduces the coherent scattering, the reflectivity, at higher angles. Below the critical angle, the reflectivity is reduced in part by the reduced transmission into the material, but, primarily, by the imaginary part of $q$. This is just the effect of extinction of the incident wave field: the high electronic scattering reduces the illumination of the nuclei in the sample.

Investigation of equation 6.18 shows it to be a distorted wave Born approximation (see chapter 5 and appendix B). Electronic multiple scattering has been included while the nuclear scattering has been added in a kinematic limit. Note that in a fully kinematic description, mentioned above, where nuclear and electronic scattering decouple, the amount of delayed nuclear scattering would be entirely independent of the electronic reflectivity. In this distorted wave approximation, the amount of nuclear scattering is dependent on the electronic scattering, but the time response is not affected. 
Looking at the time response measured from the iron layer (figures 6.9 and 6.10), the impulse response is clearly angle dependent. This indicates that either the sample is not homogeneous or dynamical considerations are important. In fact, fitting the time response shows both statements are true, however, in this case the dynamical considerations dominate. Figure 6.31 shows both the measured response and a full, dynamical calculation based on the model from fit (4), described above* . There is reasonable agreement. The figure also shows both the result of a calculation in the weak nuclear limit (e.g. equation 6.18), which is clearly seen to be a much poorer approximation, though many of the same features are present.

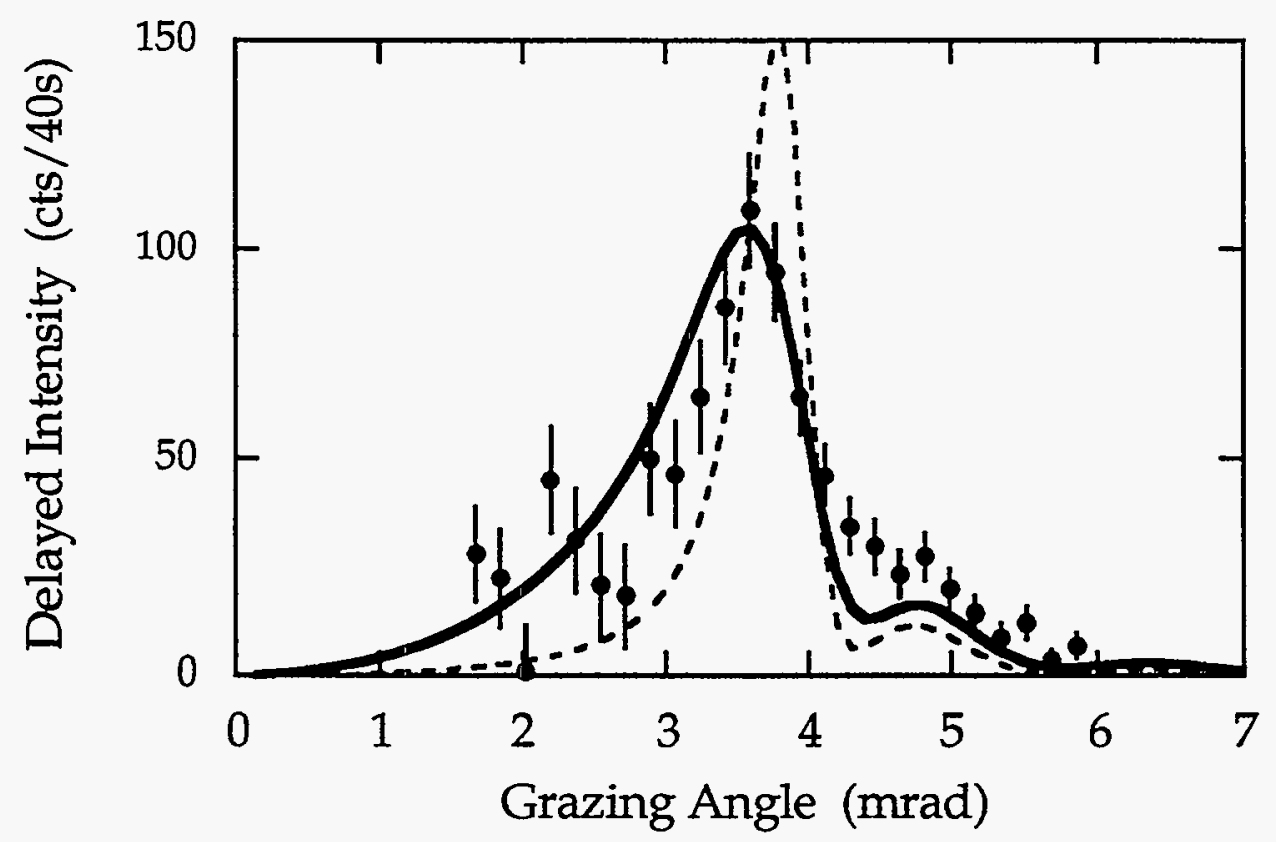

Figure 6.31. Measured delayed intensity form the thin iron layer (points) and a full dynamical scattering calculation. The dashed line shows the response in a weak nuclear limit, or distorted wave Born approximation.

\footnotetext{
* It should be pointed out that the fit in figure 6.28 is different that that of a recently published result [Baron, et al., 1994, 37]. The difference is due to the improved model of the layer presented in this work. The previous model was based on only fitting the first portion of the relfectivity curve and used roughness values that were inappropriately high.
} 


\section{Comparison with Pure Nuclear Reflections}

Figure 6.30 shows both the delayed intensity for specular reflection and that for a pure nuclear reflection, which, at grazing incidence, is the GIAR case. It is worth pointing out that although the two look very similar, in that they both peak at the electronic critical angle, there is a subtle difference between them. This is most easily seen by comparing the weak nuclear limits. For specular reflection, this is given by equation 6.17 and 6.18. For the GIAR case, it is easily seen to be (from equation 6.12)

$$
\mathrm{R}_{\mathrm{GIAR}}(\omega) \approx \frac{\delta_{\mathrm{n}}(\omega) / 2}{\theta^{2}-2 \delta_{\mathrm{e}}}
$$

In the GIAR case, the effect of the electronic scattering comes in directly as an adjustment to the angle, and the peak at the critical angle is clearly an index of refraction effect (see [Hannon, et al., 1985, 3]). This is exactly as it appears for the case of a pure nuclear Bragg reflection. However, as much of the discussion above points out, the correction in the case of specular reflection is more complicated, and in fact has rather a lot to do with the extinction of the wave field due to electronic scattering.

In the case of grazing incidence, this distinction is not as clear as one would like, because both the allowed reflection and the pure nuclear (GIAR) case lead to peaks at the same position. However, the distinction is clearer for Bragg reflections. The pure nuclear case leads to a peak at the index corrected Bragg position (see, e.g., [van Bürck, et al., 1980, 31]), as one might expect: the electronic scattering just refracts the beam entering the crystal. However, for an allowed reflection, the peak in the delayed intensity will be shifted from the Bragg position. This is shown in figure 6.32, which is completely analogous to figure 6.30 , but now for a symmetric, full, Bragg reflection, instead of grazing incidence, but with the same optical constants as used for 6.30 (and a Bragg angle of 10 degrees has been assumed). 


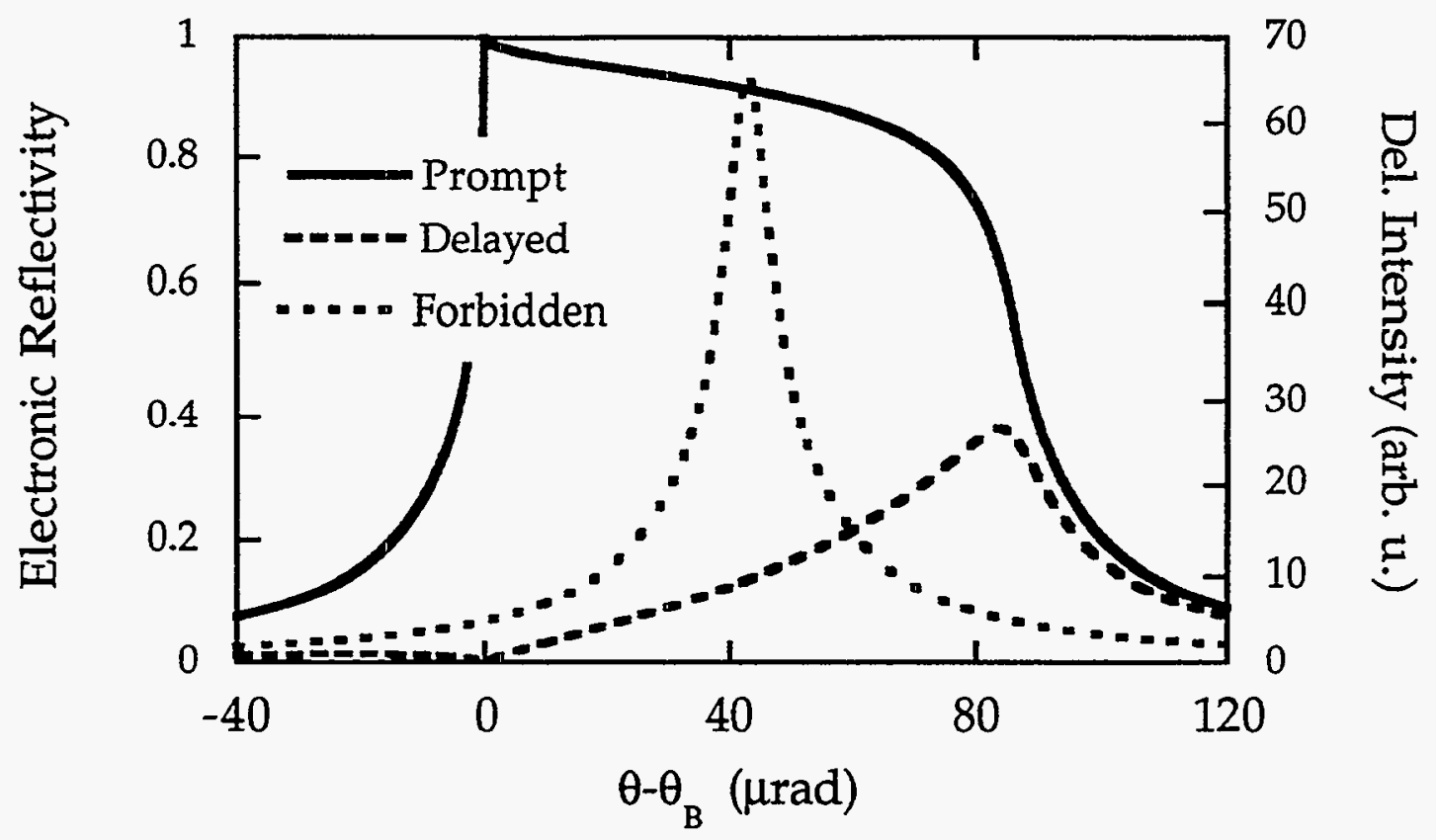

Figure 6.32 Electronic reflectivity and delayed intensity from a Bragg reflection. $X$-axis is deviation from the (uncorrected) Bragg angle. The electronic index of refraction shifts this by about 42 $\mu \mathrm{rad}$.

The following explanation is suggested. At the exact index of refraction corrected Bragg position (which occurs at the middle of the prompt or electronic peak) the extinction of the wave fields in the crystal is highest (see e.g. [Batterman and Cole, 1964, 38]) so very few nuclei are illuminated, while the wave fields penetrate further on either side this angle. However, the interplay of the incident and reflected fields inside the crystal, give rise to standing wave fields throughout the entire range of high reflectivity (Darwin width). At the low angle side of the peak, these fields have their nodes at the atomic locations, while at the high angle side, they have their maxima at the atomic locations.

Unsurprisingly, then, the nuclear contribution is largest when the field penetrates far into the crystal and is large at the locations of the nuclei, on the high angle side of the Bragg peak. 


\section{Comparison with other Mössbauer Techniques.}

The main interest in doing some sort of grazing incidence measurement along the lines of the work described in this chapter would be to gain structural information about thin films or surfaces. Therefore, it is useful to compare this work with other Mössbauer work used in this context. Indeed, in the past decade, experiments using conventional radioactive sources have addressed many interesting problems, including iron hyperfine parameters at surfaces [Korecki and Gradmann, 1985, 39], which relates to symmetry properties of the atomic environment, hyperfine parameters at interfaces [Przybylski and Gradmann, 1988, 40, Liu and Gradmann, 1993, 20], spatial oscillations in the hyperfine magnetic field [Korecki and Gradmann, 1986, 19], 2D vs 3D magnetization properties [Gutierrez, et al., 1991, 41] , magnetic field directions in multilayers [Koon, et al., 1987, 42] and interlayer coupling [Gutierrez, et al., 1991, 43] [Keavney, et al., 1993, 44]

These studies exploit the sensitivity of Mössbauer spectroscopy to monolayer or near monolayer amounts of ${ }^{57} \mathrm{Fe}$. In the case of CEMS, monolayer sensitivity is obtainable, while for transmission experiments, many layers are needed so typically a multilayer of many repeating units is used to increase the signal. The basic idea in these studies is that a sample is grown epitaxially with the ${ }^{57} \mathrm{Fe}$ placed at the location which one would like to measure. If one wished to probe a small section of an iron layer, ${ }^{56} \mathrm{Fe}$ is used for the majority, and ${ }^{57} \mathrm{Fe}$ is placed only at the probe locations [Shinjo, et al., 1977, 45] [Tyson, et al., 1981, 46].

As a structural probe, assuming one can grow many samples, these techniques are about as good as one could hope: the probe material is placed at exactly the location one wishes to look at. The only obvious improvement from using synchrotron radiation would be through its high polarization, which means the time response is very sensitive to magnetic field directions. The question, of course, would be what is the signal rate like, and this is illustrated in figure 6.33. 


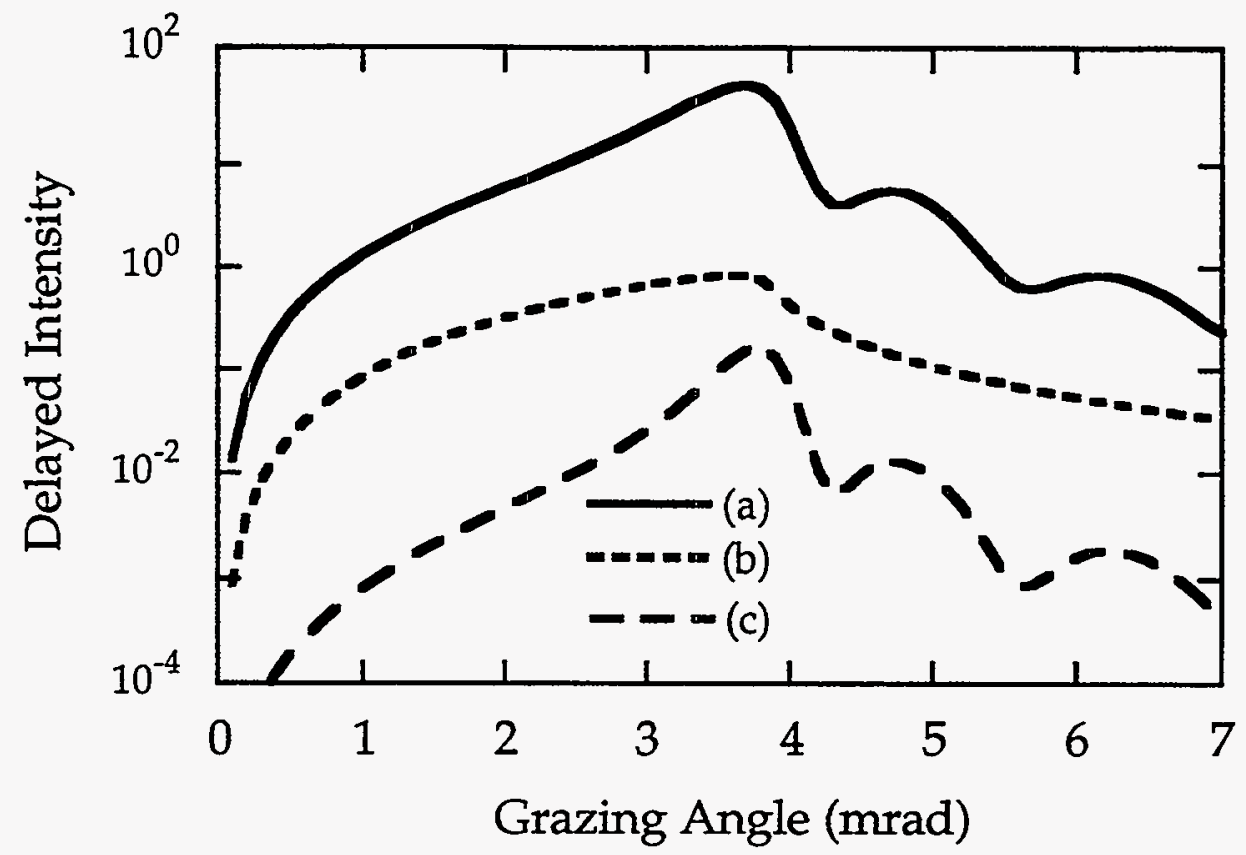

Figure 6.33. Calculated delayed intensity reflected from several samples. The vertical scale may be taken as delayed photons/reflected/s assuming an incident flux of one photon/s/natural line width. The samples are (a) the iron layer investigated in this work, (b) a monolayer of $57 \mathrm{Fe}$ deposited on top of $56 \mathrm{Fe}$ and (c) the iron layer in this work with the enrichment reduced to $2 \%$.

In order to turn the vertical scale in 6.33 into useful units, one notes that at ESRF, the undulator is predicted to provide $>10^{4} \mathrm{x}$-rays $/ \mathrm{sec} / \Gamma_{0}$. Assuming optics and other losses reduce the measured intensity by an order of magnitude, one still has a count rate of 1 photon/second at points where the delayed intensity in figure 6.33 is $10^{-3}$.

Grazing incidence measurements have been done using radioactive sources. In addition to the original work [Bernstein and Campbell, 1963, 1] [Wagner, 1968, 47] which was largely concerned with basic physics issues (i.e. interference of nuclear and electronic scattering and measuring the nuclear cross section) there has been more recent work with the goal of determining structural information about a sample. This was first done by Frost et al [Frost, et al., 1985, 35] with a highly contrived sample and has since been continued by groups in 
Russia [Andreeva, et al., 1991, 48] [Isaenko, et al., 1994, 9] and by [Röhlsberger, et al., 1993, 7]. In fact, a beautiful instrument [Irkaev, et al., 1993, 49] has been created for measuring, simultaneously, the total reflection spectrum, conversion electrons, conversion $\mathrm{x}$-ray fluorescence and re-scattered gamma-rays (see also [Andreeva, et al., 1993, 50]).

The advantage of these techniques over the probe layer techniques is that any highly enriched planar sample may be investigated with the hope of getting some structural information. The disadvantage is that, in a grazing incidence geometry with a highly enriched sample, all of the dynamical interactions discussed above come in to play so that it is necessary to understand the whole sample essentially at once. While certainly not impossible, it does make this work more difficult.

In grazing incidence work, synchrotron radiation provides essentially three advantages. First, the synchrotron radiation is highly collimated, so (up to the quality of the sample) integration over angles are not necessary, which simplifies the analysis. Second, the high linear polarization of the source means that one can easily detect variations in magnetic field directions. Finally, synchrotron radiation is much brighter than a radioactive source, so the measurement times are much shorter, and, with time gating, can be background free. However, these advantages come at the price of exchanging a direct frequency domain measurement for a time domain measurement. While in principle, the time domain measurement contains essentially the same information, it is not so easily interpreted: the appearance of an extra peak in a Mössbauer frequency spectrum can be very clear [Isaenko, et al., 1994, 9], while the equivalent modification of the beat spectrum in a time domain experiment would probably require computer fitting in order to interpret it.

One speculates, however, that an area in which synchrotron radiation experiments may outstrip the conventional source experiments is in measurement of unenriched samples. This presents very interesting possibilities, simply because any sample grown with iron could be investigated. To date, no work has been done using unenriched samples at grazing incidence, either with radioactive sources or with synchrotron radiation. However, at the 
next generation synchrotron sources, this is certainly conceivable, and would lead to low, but probably measurable signal rates (see figure 6.33 \).

It is worth pointing out another advantage of unenriched samples. In this case, one is more nearly in the distorted wave Born approximation limit discussed above, where the electronic interaction essentially determines the field profile inside the material. Thus, the electronic response could be used to determine which nuclei are illuminated, making the resonant nuclei more like a probe in a standing wave fluorescence experiment, but the coherent nuclear scattering would be measured, instead of incoherent fluorescence.

\section{Concluding Comments}

The theory of the previous two chapters has been employed to understand the specular response of a thin iron layer excited at grazing incidence. Many of the pertinent effects have been described and the theory has been shown to fit the data well. In addition, there is very clear evidence that the top portion of this sample has a different response than the bulk material, having a broader line width. Finally, what may be most interesting about this work is the extremely good quality of the data, particularly in comparison to other techniques using conventional radioactive Mössbauer sources. Thus one is very sensitive to the details of the structure. Though not fully exploited here with just one sample, there are very good prospects for performing comparative studies on larger sample sets.

\section{References for Chapter 6}

1 S. Bernstein and E.C. Campbell, Nuclear Anomalous Dispersion in Fe57 by the Method of Total Reflection. Phys. Rev. 132 (1963) 1625.

2 S. Kikuta, Y. Yoda, K. Izumi, K. Hirano, N. Horiguchi, T. Ishikawa, X.W. Zhang, H. Sugiyama, M. Ando, M. Seto, C.K. Suzuki, and S. Nasu, Nuclear resonant scattering with an $x$-ray undulator., in X-ray Resonant (Anomalous) Scattering, G. Materlik, C.J. Sparks, andK. Fischer, Editor. Amsterdam: Elsevier (1992) p. 635. 
3 J.P. Hannon, G.T. Trammell, M. Mueller, E. Gerdau, R. Rüffer, and H. Winkler, Grazing-Incidence Antireflection films III: General theory for pure nuclear reflections. Phys. Rev. B 32 (1985) 6363.

4 M. Grote, R. Röhlsberger, E. Gerdau, R. Hellmich, U. Bergmann, M. Harsdorff, M. Chambers, and W. Pfützner, Preparation and Characterization of GIARFilms for Monochromatization of Synchrotron Radiation. Hyp. Int. 58 (1989) 2439.

5 M. Grote, R. Röhlsberger, M. Dimer, E. Gerdau, R. Hellmich, R. Hollatz, J. Jäschke, E. Luken, R. Rüffer, H.D. Rüter, W. Sturhahn, E. Witthoff, M. Harsdorff, W. Pfützner, M. Chambers, and J.P. Hannon, Nuclear Resonant Filtering of Synchrotron Radiation by Grazing-Incidence Antireflection Films. Europhys. Lett. 17 (1991) 707.

6 R. Röhlsberger, E. Gerdau, M. Harsdorff, O. Leupold, E. Lüken, J. Metge, R. Rüffer, H.D. Rüter, W. Sturhahn, and E. Witthoff, Broad-band Nuclear Resonant Filters for Synchrotron Radiation: a New Source for Nuclear Diffraction Experiments. Europhys. Lett. 18 (1992) 561.

7 R. Röhlsberger, E. Gerdau, E. Lüken, H.D. Rüter, J. Metge, and O. Leupold, Nuclear diffraction experiments with grazing incidence anti-reflection films. Zeitschrift Für Physik B B92 (1993) 489.

8 E.E. Alp, T.M. Mooney, T. Toellner, W. Sturhahn, E. Witthoff, R. Röhlsberger, E. Gerdau, H. Homma, and M. Kentjana, Time Resolved Nuclear Resonant Scattering from ${ }^{119}$ Sn Nuclei using Synchrotron Radiation. Phys. Rev. Lett. 70 (1993) 3351.

9 S.A. Isaenko, A.I. Chumakov, and S.I. Shinkarev, Studies of grazing incidence reflection of nuclear gamma-radiation from 57Fe film. Physics Letters A 186 (1994) 274.

10 H. Kiessig, Ann. Phys. 10 (1931).

11 N.N. Greenwood and T.C. Gibb, Mössbauer Spectroscopy. London: Chapman and Hall, Ltd. (1971).

12 J.M. Daniels and A. Rosencwaig, Mössbauer spectroscopy of stoichimetric and non-stoichiometric magnetite. Journal of Physics and Chemstry of Solids 30 (1969) 1561.

13 C.D. Stockbridge, P.B. Sewell, and M. Cohen, An Electrometric and Electron diffraction Study of Air-Formed Oxide Films on Iron. Journal of the Electrochemical Society 108 (1961) 933. 
14 M. Langell and G.A. Somorjai, The composition and structure of oxide films grown on the (110) crystal face of iron. Journal of Vacuum Science and Technology 21 (1982) 858.

15 Z.M. Stadnik, H.R. Borsje, E.M. Swolfs, W.H.A. Leenders, and J.C. Fuggle, $A$ spherical electrostatic spectrometer for surface Mössbauer studies. Rev. Sci. Instrumen. 60 (1989) 708.

16 Y.K. Kim and M. Olivieria, Magnetic properties of sputtered Fe thin films: Processing and thickness dependence. J. Appl. Phys. 64 (1993) 1233.

17 G.N. Belozerskii, C. Bohm, T. Ekdahl, and D. Liljequist, Study of very thin surface layers by means of depth selective conversion electron Mössbauer spectrocopy (DCEMS). Nucl. Instrumen. and Meth. 192 (1982) 539.

18 R. Droste, G. Stern, and J.C. Walker, Magnetic Hyperfine Fields at Fe Surface and Interfaces. Journal of Magnetism and Magnetic Materials 54-57 (1986) 763.

19 J. Korecki and U. Gradmann, Spatial Oscillation of Magnetic Hyperfine Field near Free Fe(110)-Surface. Europhys. Lett. 2 (1986) 651.

20 G. Liu and U. Gradmann, Magnetic Order Near Fe(100) interfaces from Mössbauer spectroscopy. Journal of Magnetism and Magnetic Materials 118 (1993) 99.

21 T.S. Toellner, T. Mooney, S. Shastri, and E.E. Alp. High energy resolution, high angular acceptance crystal monochromator. in Optics for High-Brightness Synchrotron Beamlines. J. Arthur ed. SPIE Vol 1740, 1992. p. 218.

22 U. Bergmann, S.D. Shastri, D.P. Siddons, B.W. Batterman, and J.B. Hastings, Temperature dependence of nuclear forward scattering of synchrotron radiation in alpha- ${ }^{57}$ Fe. Phys. Rev. B 50 (1994) 5957.

23 S.S. Hanna, J. Herberle, G.J. Perlow, R.S. Preston, and D.H. Vincent, Direction of the Effective Magnetic Field at the Nucleus in Ferromagnetic Iron. Phys. Rev. Lett. 4 (1960) 513.

24 M. Prutton, Thin Ferromagnetic Films. London: Butterworth and Co. (1964).

25 D.E. Brown, J. Arthur, A.Q.R. Baron, G.S. Brown, and S. Shastri, Phase Shift of a Rotated Quantum State Observed in an X-Ray Scattering Experiment. Phys. Rev. Lett. 69 (1992) 699. 
26 D.Y. Smith and J.H. Barykoumb, Sign reoersal of the atomic scattering factor and grazing-incidence transmission at $x$-ray-absorption edges. Phys. Rev. B 41 (1990) 11529.

27 G.T. Trammell, Gamma Ray Diffraction by Resonant Nuclei, in Chemical Effects on Nuclear Transformations, Editor. Vienna: International Atomic Energy Agency (1961) p. 75.

28 Y. Kagan, A.M. Afanas'ev, and I.P. Perstnev, Theory of Resonance Bragg Scattering of $\gamma$ Quanta by Regular Crystals. Zh. Eksp. Teor. Fiz. [Sov. Phys. JETP 27 (1968) 819] 54 (1968) 1530.

29 J.P. Hannon and G.T. Trammell, Mössbauer Diffraction. II. Dynamical Theory of Mössbauer Optics. Phys. Rev. 186 (1969) 306.

30 Y. Kagan, A.M. Afanas'ev, and V.G. Kohn, On Excitation of Isomeric Nuclear States in a Crystal by Synchrotron Radiation. J. Phys. C 12 (1979) 615.

31 U. van Bürck, G.V. Smirnov, R.L. Mössbauer, H.J. Marus, and N.A. Semioschkina, Enhanced Nuclear Resonance Scattering in Dynamical Diffraction of Gamma Rays. J. Phys. C 13 (1980) 4511.

32 J.P. Hannon, G.T. Trammell, M. Mueller, E. Gerdau, R. Rüffer, and H. Winkler, Grazing-Incidence Antireflection Films. IV. Application to Mössbauer Filtering of Synchrotron Radiation. Phys. Rev. B32 (1985) 6374.

33 Numerical Algorithms Group, NAG Fortran Library, Mark 15. NAG Ltd. Wilkinson House, Jordan Hill Road, OXFORD, UK OX2 8DR.

34 C.R. Brundle, Core-level Photoemission and LEED Studies of Adsoption at Fe Surfaces: Comparison Bewteen $\mathrm{CO}$ and $\mathrm{O}_{2}$. IBM Journal of Research and Devellopment 22 (1978) 235.

35 J.C. Frost, B.C.C. Cowie, S.N. Chapman, and J.F. Marshall, Surface Sensitive Mössbauer spectroscopy by the combination of total external reflection and conversion electron detection. Appl. Phys. Lett. 47 (1985) 581.

36 S.M. Irkaev, M.A. Andreeva, V.G. Semenov, G.N. Belozerskii, and O.V. Grishin, Grazing incidence Mössbauer spectroscopy: new method for surface layers analysis. Part I. Instrumentation. Nuclear Instruments and Methods in Physics Research B74 (1993) 545.

37 A.Q.R. Baron, J. Arthur, S.L. Ruby, A.I. Chumakov, G.V. Smirnov, and G.S. Brown, Angular dependence of specular resonant nuclear scattering of $x$-rays. Phys. Rev. B 50 (1994) 10354. 
38 B.W. Batterman and H. Cole, Dynamical Diffraction of X-Rays by Perfect Crystals. Rev. Mod. Phys. 36 (1964) 681.

39 J. Korecki and U. Gradmann, In Situ Mössbauer Analysis of Hyperfine Interactions near Fe(110) Surfaces and Interfaces. Phys. Rev. Lett. 55 (1985) 2491.

40 M. Przybylski and U. Gradmann, CEMS Analysis of Ferromagnetic Interfaces. Hyp. Int. 41 (1988) 693.

41 C.J. Gutierrez, Z.Q. Qiu, M.D. Wieczorek, H.Tang, and J.C. Walker, The observation of a 3-D to 2-D crossover in the magnetization of Epitaxial $\mathrm{Fe}(110) / \mathrm{Ag}$ (111) multilayers. Journal of Magnetism and Magnetic Materials 93 (1991) 336.

42 N.C. Koon, B.T. Jonker, F.A. Volkening, J.J. Krebs, and G.A. Prinz, Direct Evidence for Perpendicular Spin Orientations and Enhanced Hyperfine Fields in Ultrathin Fe(100) Films on Ag(100). Phys. Rev. Lett. 59 (1987) 2463.

43 C.J. Gutierrez, Z.Q. Qiu, M.D. Wieczorek, H.Tang, J.C. Walker, and R.C. Mercader, Mössbauer Studies of Spin Wave Excitations in Fe/Ag Multilayers. Hyp. Int. 66 (1991) 299.

44 D.J. Keavney, D.F. Storm, J.W. Freeland, M.D. Wieczoreki, J.C. Walker, M.G. Pini, P. Politi, and A. Rettori, Oscillatory exchange coupling of ferromagnetically aligned Fe(110) layers through Ag(111) interlayers. Phys. Rev. Lett. 71 (1993) 927.

45 T. Shinjo, S. Hine, and T. Takada. . in 7 th international Vacuum Conference and 3rd International Conference on Solid Surfaces. 1977. Vienna p. 2655.

46 J. Tyson, A.H. Owens, J.C. Walker, and G. Bayreuther, Increase in magnetic hyperfine fields at the surface of ultra-thin epitaxial Fe films. J. Appl. Phys. 52 (1981) 2487.

47 F.E. Wagner, Totalreflexion der rückstossfreien $8.4 \mathrm{keV}$ g-Strahlung des Tm169. Zeitscrift für Physic 210 (1968) 361.

48 M.A. Andreeva, G.N. Belozerskii, S.M. Irkaev, V.G. Semenov, A.Y. Sokolov, and N.V. Shumilova, Investigation of thin oxide 57Fe films by Mossbauer total external reflection. Physica Status Solidi A 127 (1991) 455.

49 S.M. Irkaev, M.A. Andreeva, V.G. Semenov, G.N. Belozerskii, and O.V. Grishin, Grazing incidence Mössbauer spectroscopy: new method for surface 
layers analysis. Part II. Theory of grazing incidence Mössbauer spectra. Nuclear Instruments and Methods in Physics Research B74 (1993) 554.

50 M.A. Andreeva, G.N. Belozerski, O.V. Grishin, S.M. Irkaev, and V.G. Semenov, Mössbauer Total External Reflection, 2: Four Scattering Channels. Presented at the 1993 International Conference on Applications of the Mössbauer Effect, To Be Published in Hyperfine Interactions (1993). 


\section{Concluding Comments}

Nuclear resonant scattering experiments using synchrotron radiation are fascinating because they combine conventional $x$-ray scattering techniques and ideas with the effects of scattering from the extremely narrow nuclear resonance. Furthermore, the polarization selectivity of the resonant scattering and the time domain measurement of the response add a layer of subtlety to these measurements that enhances their appeal, leading to interesting and surprising results, and new ways of thinking.

Chapter two of this thesis introduces some of the ideas that are important in understanding the field, both on a conceptual level and a practical one. The comparison of the response of a simple absorber in the time domain and the frequency domain serves to illustrate some of the differences between these types of measurements, pleasantly permitting the application and demonstration of some basic physical principles. Most importantly, a collection of nuclei is seen to behave rather differently than an individual nucleus.

The broad bandwidth of synchrotron radiation has driven the development of sophisticated $x$-ray optical elements in order to reduce the nonresonant background in nuclear scattering experiments. High order Bragg reflections and asymmetrically cut crystals allow the reduction of the background rate by 3 orders of magnitude while largely preserving the signal rate. Thus, with a sufficiently good detector, many of the techniques commonly used in x-ray scattering may be applied to resonant nuclear scattering.

It is necessary to have a good detector, however, and much of this thesis work has been focused on making such an object. A microchannel plate (MCP) detector offered the possibility to do extremely high count rate experiments (perhaps without the use of the sophisticated optical techniques mentioned above) but finally proved to be rather complicated to operate. The idea was shown to be feasible, and some investigation of $\mathrm{x}$-ray photocathodes was done, showing that efficiencies of $\sim 50 \%$ were possible in a grazing incidence geometry with Au or CsI photocathodes at $14.4 \mathrm{keV}$. With the development of the 
asymmetric nested monochromator, the complexity of the MCP detector became unnecessary. Thus, the work with avalanche photodiodes (APD's) was begun, finally leading to detectors capable of operating at very high count rates $\left(10^{6}-10^{8}\right.$ photons/sec) with sub nanosecond time resolution and good efficiencies (30$70 \%)$.

The bulk of the thesis investigates specular scattering from layered materials containing resonant nuclei. Grazing incidence specular reflection measurements are a common conventional $x$-ray scattering technique but had not been studied with resonant transitions in time domain measurements. In particular, there was a need for experimental work in which the time domain response was directly investigated and for some additional theoretical analysis. Accordingly, a careful derivation of the expected response in specular reflection from layered structures was carried out, beginning with Maxwell's equations and relying of the Lorentz relation to connect to the quantum mechanics of the interaction of individual nuclei. This treatment explicitly included the polarization effects that can strongly influence nuclear scattering. In addition, careful consideration was made of the effect that interfacial roughness has on the specular response, with the extension of techniques from conventional (electronic) $x$-ray scattering to polarization dependent nuclear scattering. A crucial point in this analysis is the difference between a kinematic and a dynamical $x$-ray scattering limit. The kinematic limit is much simpler, but, in general, not applicable to nuclear scattering situations, particularly not at grazing incidence.

Analysis of the specular response measured from a thin layer of ${ }^{57} \mathrm{Fe}$ deposited on glass served to test the theory developed in this work. The theory was shown to be in good agreement with the data and indicated that the nuclear response of the surface was different than that of the bulk of the layer. Also, the effects of dynamical scattering on the time response were discussed at length, showing, in particular, how dynamical scattering could shift the beat frequencies expected from simple kinematic theory, how it could change the contrast between the beats and also how it might introduce new frequencies into the time response. In addition, investigation of the total (integrated) nuclear scattered radiation showed that the effects of extinction of the wave field due to electronic 
scattering must be included to understand the response in the allowed reflection, with interesting implications for finite order allowed Bragg reflections.

Future work might be expected in several directions. Detector development is never really complete, with new experiments requiring at least small upgrades in detection systems. In this case, the obvious directions for improvements are compensation or removal of the baseline shift in the output at high rates, and, maybe, improvement of the amplification scheme (reducing the noise). As for specular reflection measurements, having completed a proof of principle, one might consider experiments more along the lines of materials science. However, in this context one must determine what information these techniques might provide that is not more readily available using other methods. This is a broad subject and one only notes here that, in general, there are many techniques that may be used to look at surfaces, and $x$-rays sometimes find their niche looking a buried interfaces in multilayer structures, for which there are fewer direct probes. This also alleviates concerns of sample contamination outside of a UHV environment. Finally, more along the lines of interesting basic physics, one might explore allowed Bragg reflections in perfect crystals or multilayer materials, using the electronic scattering to determine the illumination of the nuclei (e.g. the distorted wave Born approximation discussed at the end of chapter 6), much like some $x$-ray standing wave measurements. 


\section{Appendix A: The Forward Scattering Amplitude}

\section{Introduction}

The scattering amplitude mentioned in chapter 4 is, with the Lorentz relation, the interface between the quantum mechanical treatment of $x$-rays interacting with nuclei and the Maxwell equations for macroscopic materials. A derivation of this quantity based on the work of many authors (in particular, [Frauenfelder, et al., 1962, 1], [Blume and Kistner, 1968, 2] and [Hannon and Trammell, 1969, 3])has been presented for the case at hand in the thesis of D.E. Brown [Brown, 1993, 4],. For the purposes of the work here, I simply quote the form for general multipole scattering and then consider specifically the case of magnetic dipole scattering, as is important for the $14.4 \mathrm{keV}$ transition in $57 \mathrm{Fe}$. Several special cases are investigated in detail.

\section{Multipole Scattering Amplitude}

The elastic nuclear scattering amplitude may be written ([Hannon and Trammell, 1969, 3] and [Brown, 1993, 4], p 58) for a well defined multipole transition

$$
\begin{aligned}
& \mathrm{F}_{n}\left(\mathbf{k}_{\mathrm{i}} \rightarrow \mathbf{k}_{\mathrm{f}}\right)=-\frac{2 \pi}{\mathrm{k}_{\mathrm{f}}} \sum_{\mathrm{LIM}} \sum_{\mathrm{L}^{\prime} \mathrm{I}^{\prime} \mathrm{M}^{\prime}}\left[\hat{\mathbf{e}}_{\mathrm{f}}^{*} \cdot \mathrm{Y}_{\mathrm{LIM}}\left(\Omega_{\mathbf{k}_{\mathrm{f}}}\right)\right]\left[\hat{\mathbf{e}}_{\mathrm{i}} \bullet \mathrm{Y}_{\mathrm{L}^{\prime} \mathrm{I}^{\prime} \mathrm{M}^{\prime}}^{*}\left(\Omega_{\mathbf{k}_{\mathrm{i}}}\right)\right] \\
& x \frac{\left\langle j_{f} L m_{f} M \mid j_{f} L j_{n} m_{n}\right\rangle\left\langle j_{i} L^{\prime} m_{i} M^{\prime} \mid j_{i} L^{\prime} j_{n} m_{n}\right\rangle}{E_{i}-E_{n}+\hbar \omega_{k_{i}}+i \Gamma_{n} / 2} \\
& x \quad \sqrt{\Gamma(L, 1) \Gamma\left(L^{\prime}, l^{\prime}\right)} e^{i\left[\eta(L, l)-\eta\left(L^{\prime}, l^{\prime}\right)\right]}
\end{aligned}
$$

The subscript $\mathrm{n}$ indicates the nuclear intermediate state under consideration and will be summed over. The incident photon is assumed to have wave vector $\mathbf{k}_{\mathbf{i}}$ and polarization $\hat{\mathbf{e}}_{\mathbf{i}}$. The scattered photon has wave vector $\mathbf{k}_{\mathbf{f}}$ and polarization $\hat{\mathbf{e}}_{\mathrm{f}}$. The nucleus is assumed to have initial state $\left|j_{i} m_{i}\right\rangle$, intermediate state $\left|j_{n} m_{n}\right\rangle$ and final state $\left|j_{f} m_{f}\right\rangle$, where the form of each is the total spin, $j$, and projection of 
the spin along a quantization axis, $\mathrm{m} . \Gamma_{\mathrm{n}}$ is the total line width of the intermediate nuclear state and $\Gamma(L, 1)$ are the partial line widths for radiative transitions. The multipolarity of the transition is determined by $\mathrm{L}, 1, \mathrm{~L}$ ' and $\mathrm{l}^{\prime}$. The terms in the brackets of the form $\left\langle\mathrm{j}_{1} \mathrm{j}_{2} \mathrm{~m}_{1} \mathrm{~m}_{2} \mid \mathrm{j}_{\mathrm{j}} \mathrm{j}_{2} \mathrm{jm}\right\rangle$ are Clebsch Gordon coefficients and will be zero unless $m_{1}+m_{2}=m$. Thus $M=m_{n}-m_{f}$ and $M^{\prime}=m_{n}-m_{i}$. The $\mathbf{Y}_{\mathrm{LM}}$ are vector spherical harmonics and may be defined by

$$
\mathrm{Y}_{\mathrm{LIM}}=\sum_{\mathrm{mq}}\langle 11 \mathrm{mq} \mid 11 \mathrm{LM}\rangle \mathrm{Y}_{\mathrm{Im}} \hat{\mathbf{e}}_{\mathrm{q}}
$$

where the $Y_{\operatorname{lm}}$ are the standard spherical harmonics and $\hat{\mathbf{e}}_{\mathrm{q}}$ are the spherical basis unit vectors. See [Weissbluth, 1978, 5] chapter 7.

The formula A.1 is appropriate for any form of energy conserving scattering. We shall be concerned only with coherent elastic scattering, which leaves the nuclear system unchanged. Thus $\left|j_{i} m_{i}\right\rangle=\left|j_{f} m_{f}\right\rangle$ and one has $L=L^{\prime}$ and $\mathrm{M}=\mathrm{M}^{\prime}$. Furthermore, we shall only consider transition with a single well defined multipole character, so that $l^{\prime}=1$. The expression for the scattering amplitude simplifies to

$$
\begin{aligned}
F_{n}\left(\mathbf{k}_{i} \rightarrow \mathbf{k}_{\mathrm{f}}\right)= & -\frac{2 \pi}{k_{\mathrm{f}}} \sum_{\substack{\mathrm{Ll} \\
\mathrm{M}=\mathrm{m}_{\mathrm{n}}-\mathrm{m}_{\mathrm{i}}}}\left[\hat{\mathbf{e}}_{\mathrm{f}}^{*} \cdot \mathrm{Y}_{\mathrm{LIM}}\left(\Omega_{\mathbf{k}_{\mathrm{f}}}\right)\right]\left[\hat{\mathbf{e}}_{\mathrm{i}} \cdot \mathrm{Y}_{\mathrm{LIM}}^{*}\left(\Omega_{\mathbf{k}_{\mathrm{i}}}\right)\right] \\
& \times \frac{\Gamma_{\mathrm{r}}(\mathrm{L}, 1)\left(\mathrm{j}_{\mathrm{i}} L \mathrm{~m}_{\mathrm{i}} \mathrm{M} \mid \mathrm{j}_{\mathrm{i}} \mathrm{Lj} \mathrm{j}_{\mathrm{n}} \mathrm{m}_{\mathrm{n}}\right)^{2}}{\mathrm{E}_{\mathrm{i}}-\mathrm{E}_{\mathrm{n}}+\hbar \omega_{\mathbf{k}_{\mathrm{i}}}+\mathrm{i} \Gamma_{\mathrm{n}} / 2}
\end{aligned}
$$

\section{Magnetic Dipole Transition}

Specializing to the magnetic dipole transition in $57 \mathrm{Fe}$, we require $\mathrm{L}=\mathrm{l}=1$. Including the sum over intermediate nuclear states, $n$, the scattering amplitude then becomes 


$$
\begin{aligned}
\mathrm{F}_{\text {nuc }}\left(\mathrm{m}_{\mathrm{i}}, \mathbf{k}_{\mathrm{i}} \rightarrow \mathrm{k}_{\mathrm{f}}\right)= & -\frac{2 \pi}{\mathrm{k}} \frac{\Gamma_{\mathrm{r}}}{\Gamma_{0}} \sum_{\mathrm{M}=0, \pm 1}\left[\hat{\mathbf{e}}_{\mathrm{f}}^{*} \cdot \mathrm{Y}_{11 \mathrm{M}}\left(\Omega_{\mathrm{k}_{\mathrm{f}}}\right)\right]\left[\hat{\mathbf{e}}_{\mathrm{i}} \cdot \mathrm{Y}_{11 \mathrm{M}}^{*}\left(\Omega_{\mathrm{k}_{\mathrm{i}}}\right)\right] \\
& \times \frac{\left\langle\frac{1}{2} 1 \mathrm{~m}_{\mathrm{i}} \mathrm{M} \mid \frac{1}{2} 1 \frac{3}{2} \mathrm{M}+\mathrm{m}_{\mathrm{i}}\right\rangle^{2}}{\left(\mathrm{E}_{\mathrm{i}}-\mathrm{E}_{\mathrm{n}}+\hbar \omega\right) / \Gamma_{0}+\mathrm{i} / 2}
\end{aligned}
$$

where $\Gamma_{0}$ is the natural line width of the $14.4 \mathrm{keV}$ transition in ${ }^{57} \mathrm{Fe}$ and $\Gamma_{\mathrm{r}}$ is the radiative line width. The initial angular momentum is $\mathrm{j}_{\mathrm{i}}=1 / 2$ while the excited state has angular momentum $j_{n}=3 / 2$. The spin projection of the initial nuclear state is $m_{i}= \pm 1 / 2$. Finally, specializing to the case of forward scattering and assuming equal probability for the two ground states (which is a good approximation at room temperature since the energy splittings are much less then $k_{B} T$ ) one has

$$
\begin{aligned}
\mathrm{F}_{\text {nuc }}\left(\hat{\mathbf{k}}, \omega, \hat{\mathbf{e}}_{\mathrm{i}} \rightarrow \hat{\mathbf{e}}_{\mathrm{f}}\right)= & -\frac{2 \pi}{\mathrm{k}} \frac{\Gamma_{\mathrm{r}}}{\Gamma_{0}} \sum_{\mathrm{M=0, \pm 1}}\left[\hat{\mathbf{e}}_{\mathrm{f}}^{*} \bullet \mathbf{Y}_{11 \mathrm{M}}\left(\Omega_{\mathbf{k}}\right)\right]\left[\hat{\mathbf{e}}_{\mathrm{i}} \bullet \mathbf{Y}_{11 \mathrm{M}}^{*}\left(\Omega_{\mathbf{k}}\right)\right] \\
& \times \frac{1}{2} \sum_{\mathrm{m}_{\mathrm{i}}= \pm 1 / 2} \frac{\left\langle\frac{1}{2} 1 \mathrm{~m}_{\mathrm{i}} \mathrm{M} \mid \frac{1}{2} 1 \frac{3}{2} \mathrm{M}+\mathrm{m}_{\mathrm{i}}\right\rangle^{2}}{\left(\mathrm{E}_{\mathrm{i}}-\mathrm{E}_{\mathrm{n}}+\hbar \omega\right) / \Gamma_{0}+\mathrm{i} / 2}
\end{aligned}
$$

The polarization dependence of the above expression may be explicitly evaluated by selecting an orthonormal polarization basis to describe the scattering. In this case the nuclear scattering amplitude may be considered to be a $2 \times 2$ matrix relating the incident and final polarizations. In particular one writes

$$
\overrightarrow{\mathrm{F}}_{\text {nuc }}(\hat{\mathbf{k}}, \omega)=-\frac{2 \pi}{\mathrm{k}} \frac{\Gamma_{\mathrm{r}}}{\Gamma_{0}} \sum_{\mathrm{M}=0, \pm 1} \overrightarrow{\mathrm{P}}_{\mathrm{M}}(\hat{\mathbf{k}}) \frac{1}{2} \sum_{\mathrm{m}_{\mathrm{i}}= \pm 1 / 2} \frac{\left\langle\frac{1}{2} 1 \mathrm{~m}_{\mathrm{i}} \mathrm{M} \mid \frac{1}{2} 1 \frac{3}{2} \mathrm{M}+\mathrm{m}_{\mathrm{i}}\right\rangle^{2}}{\left(\mathrm{E}_{\mathrm{i}}-\mathrm{E}_{\mathrm{n}}+\hbar \omega\right) / \Gamma_{0}+\mathrm{i} / 2}
$$

where

$$
\overrightarrow{\mathbf{P}}_{M}(\hat{\mathbf{k}}) \equiv\left(\begin{array}{ll}
\left(\hat{\mathbf{e}}_{1}^{*} \bullet \mathbf{Y}_{11 M}(\hat{\mathbf{k}})\right)\left(\hat{\mathbf{e}}_{1} \bullet \mathbf{Y}_{11 M}^{*}(\hat{\mathbf{k}})\right) & \left(\hat{\mathbf{e}}_{1}^{*} \bullet \mathbf{Y}_{11 M}(\hat{\mathbf{k}})\right)\left(\hat{\mathbf{e}}_{2} \bullet \mathbf{Y}_{11 M}^{*}(\hat{\mathbf{k}})\right) \\
\left(\hat{\mathbf{e}}_{2}^{*} \cdot \mathbf{Y}_{11 M}(\hat{\mathbf{k}})\right)\left(\hat{\mathbf{e}}_{1} \cdot \mathbf{Y}_{11 M}^{*}(\hat{\mathbf{k}})\right) & \left(\hat{\mathbf{e}}_{2}^{*} \cdot \mathbf{Y}_{11 M}(\hat{\mathbf{k}})\right)\left(\hat{\mathbf{e}}_{2} \bullet \mathbf{Y}_{11 M}^{*}(\hat{\mathbf{k}})\right)
\end{array}\right)
$$


The $\hat{\mathbf{e}}_{\mathrm{i}}$ are some basis in which we have chosen to express the scattering amplitude. Taking the quantization axis to be the $\mathrm{z}$ axis, one may write (see e.g. Weissbluth, table 7.1)

$$
\begin{aligned}
& Y_{111}^{\mathbf{z}}(\theta, \varphi)=\sqrt{\frac{3}{16 \pi}}\left(\cos \theta, \mathrm{i} \cos \theta,-\sin \theta \mathrm{e}^{\mathrm{i} \varphi}\right) \\
& \mathbf{Y}_{110}^{\hat{\mathrm{z}}}(\theta, \varphi)=\sqrt{\frac{3}{8 \pi}}(-\mathrm{i} \sin \theta \sin \varphi, \mathrm{i} \sin \theta \cos \varphi, 0) \\
& \mathrm{Y}_{11-1}^{\hat{\mathrm{z}}}(\theta, \varphi)=\mathrm{Y}_{11-1}(\theta, \varphi)^{*}
\end{aligned}
$$

The angles $\theta$ and $\varphi$ are the polar and azimuthal angles, respectively and the three components are in the $\mathrm{x}, \mathrm{y}$ and $\mathrm{z}$ directions. The superscript indicates the direction of the quantization axis. We will want to consider different orientations of the quantization axis (for fixed photon direction and polarization) so it is useful to generalize this. In particular, taking the rotation operator $\overline{\mathbf{R}}_{\hat{\mathbf{q}}}$ to be defined by

$$
\overrightarrow{\mathbf{R}}_{\hat{\mathbf{q}}} \hat{\mathbf{z}}=\hat{\mathbf{q}}
$$

one has (since the vector spherical harmonics transform as vectors)

$$
\mathbf{Y}_{11 M}^{\hat{\mathrm{q}}}(\hat{\mathbf{k}})=\overrightarrow{\mathbf{R}}_{\hat{\mathbf{q}}} \mathbf{Y}_{11 M}^{\hat{\mathbf{z}}}\left(\overrightarrow{\mathbf{R}}_{\hat{\mathbf{q}}}^{-1} \hat{\mathbf{k}}\right)
$$

The scalar dot product in A.7 may then be evaluated

$$
\hat{\mathbf{e}}_{\mathrm{i}}^{*} \bullet \mathbf{Y}_{11 \mathrm{M}}^{\hat{\mathrm{q}}}(\hat{\mathbf{k}})=\hat{\mathbf{e}}_{\mathrm{i}}^{*} \bullet \overrightarrow{\mathbf{R}}_{\hat{\mathbf{q}}} \mathbf{Y}_{11 \mathrm{M}}^{\hat{2}}\left(\overrightarrow{\mathbf{R}}_{\hat{\mathbf{q}}}^{-1} \hat{\mathbf{k}}\right)=\overrightarrow{\mathbf{R}}_{\hat{\mathbf{q}}}^{-1} \hat{\mathbf{e}}_{i}^{*} \bullet \mathbf{Y}_{11 M}^{\hat{\imath}}\left(\overrightarrow{\mathbf{R}}_{\hat{\mathbf{q}}}^{-1} \hat{\mathbf{k}}\right)
$$

We can explicitly write down an appropriate rotation matrix ${ }^{*}$

$$
\overrightarrow{\mathbf{R}}_{\hat{\mathbf{q}}}^{-1}=\left(\begin{array}{ccc}
\cos \theta_{\hat{\mathbf{q}}} \cos \varphi_{\hat{\mathbf{q}}} & \cos \theta_{\hat{\mathbf{q}}} \sin \varphi_{\hat{\mathbf{q}}} & -\sin \theta_{\hat{\mathbf{q}}} \\
-\sin \varphi_{\hat{\mathbf{q}}} & \cos \varphi_{\hat{\mathbf{q}}} & 0 \\
\sin \theta_{\hat{\mathbf{q}}} \cos \varphi_{\hat{\mathbf{q}}} & \sin \theta_{\hat{\mathrm{q}}} \sin \varphi_{\hat{\mathbf{q}}} & \cos \theta_{\hat{\mathbf{q}}}
\end{array}\right)
$$

\footnotetext{
* Equation (A.15) does not uniquely determine the rotation matrix. However, it is sufficient just to choose a well defined set. I simply choose the Euler rotation matrix where $\alpha=0$ and $\beta$ and $\gamma$ have been set to the polar and azimuthal angles of the quantization axis. See [Weissbluth, 1978, 5] p. 55.
} 
where $\theta_{\hat{\mathbf{q}}}$ and $\varphi_{\hat{\mathbf{q}}}$ are the angles that the quantization axis makes with the $z$ axis. Choosing the photon propagation directions and polarizations to be $\hat{\mathbf{k}}=\hat{\mathbf{z}}, \hat{\mathbf{e}}_{1}=\hat{\mathbf{x}}$ and $\hat{\mathbf{e}}_{2}=\hat{\mathbf{y}}$, one has

$$
\begin{aligned}
& \overrightarrow{\mathbf{p}} 1=\frac{3}{16 \pi}\left(\begin{array}{c}
\cos ^{2} \varphi_{\hat{\mathbf{q}}}+\sin ^{2} \varphi_{\hat{\mathbf{q}}} \cos ^{2} \theta_{\hat{\mathbf{q}}} \\
\sin ^{2} \theta_{\hat{\mathbf{q}}} \cos \varphi_{\hat{\mathbf{q}}} \sin \varphi_{\hat{\mathbf{q}}}+i \cos \theta_{\hat{\mathbf{q}}}
\end{array}\right. \\
& \sin ^{2} \theta_{\hat{\mathbf{q}}} \cos \varphi_{\hat{\mathbf{q}}} \sin \varphi_{\hat{\mathrm{q}}}-\mathrm{i} \cos \theta_{\hat{\mathbf{q}}} \\
& \left.\sin ^{2} \varphi_{\hat{\mathrm{q}}}+\cos ^{2} \varphi_{\hat{\mathrm{q}}} \cos ^{2} \theta_{\hat{\mathbf{q}}}\right) \\
& \overrightarrow{\mathbf{P}}_{0}^{\hat{\mathbf{q}}}=\frac{3}{8 \pi}\left(\begin{array}{cc}
\sin ^{2} \varphi_{\hat{\mathbf{q}}} \sin ^{2} \theta_{\hat{\mathbf{q}}} & \sin ^{2} \theta_{\hat{\mathbf{q}}} \cos \varphi_{\hat{\mathbf{q}}} \sin \varphi_{\hat{\mathbf{q}}} \\
\sin ^{2} \theta_{\hat{\mathbf{q}}} \cos \varphi_{\hat{\mathbf{q}}} \sin \varphi_{\hat{\mathbf{q}}} & \cos ^{2} \varphi_{\hat{\mathbf{q}}} \sin ^{2} \theta_{\hat{\mathbf{q}}}
\end{array}\right) \\
& \overrightarrow{\mathbf{P}_{-1}^{\hat{q}}}=\overrightarrow{\mathbf{P}}_{1}^{\hat{\mathrm{q}}^{*}}
\end{aligned}
$$

where the star superscript in (A.13c) indicates that the complex conjugate should be taken of each element. We have also made use of the fact that $\mathbf{Y}_{11 \mathrm{M}}^{\hat{\mathbf{z}}}\left(\overrightarrow{\mathbf{R}}_{\hat{\mathbf{q}}}^{-1} \hat{\mathbf{z}}\right)=\mathbf{Y}_{11 \mathrm{M}}^{\hat{\mathbf{z}}}\left(\theta=-\theta_{\hat{\mathbf{q}}}, \varphi=0\right)$.

If we wish to consider the interaction of a with a sample with a single, well defined quantization direction (as opposed to a distribution of such directions that might occur in an unmagnetized iron foil), one can choose the polarization basis so that the quantization axis is in the $x z$ plane. In this case $\varphi_{\hat{q}}=0$ and polarization matrices reduce to simpler forms:

$$
\begin{aligned}
& \overrightarrow{\mathbf{P}}_{1}(\hat{\mathbf{k}})=\frac{3}{16 \pi}\left(\begin{array}{cc}
1 & -i \cos \theta \\
i \cos \theta & \cos ^{2} \theta
\end{array}\right) \\
& \overrightarrow{\mathbf{P}}_{0}(\hat{\mathbf{k}})=\frac{3}{8 \pi}\left(\begin{array}{cc}
0 & 0 \\
0 & \sin ^{2} \theta
\end{array}\right) \\
& \overrightarrow{\mathbf{P}}_{-1}(\hat{\mathbf{k}})=\frac{3}{16 \pi}\left(\begin{array}{cc}
1 & i \cos \theta \\
-i \cos \theta & \cos ^{2} \theta
\end{array}\right)
\end{aligned}
$$


Finally, explicitly writing out the sums in (A.6) and evaluating the ClebschGordon coefficients gives the result:

$$
\begin{aligned}
& \overrightarrow{\mathbf{F}}_{\text {nuc }}(\hat{\mathbf{k}}, \omega)=-\frac{2 \pi}{\mathrm{k}} \frac{\Gamma_{\mathrm{r}}}{\Gamma_{0}} \mathrm{x} \\
& \quad\left\{\overrightarrow{\mathbf{P}}_{1}(\theta)\left(\frac{1}{2\left(\hbar \omega-\mathrm{E}_{3 / 2 \rightarrow 1 / 2}\right) / \Gamma_{0}+\mathrm{i}}+\frac{1 / 3}{2\left(\hbar \omega-\mathrm{E}_{1 / 2 \rightarrow-1 / 2}\right) / \Gamma_{0}+\mathrm{i}}\right)\right. \\
& +\overrightarrow{\mathbf{P}}_{0}(\theta)\left(\frac{2 / 3}{2\left(\hbar \omega-\mathrm{E}_{1 / 2 \rightarrow 1 / 2}\right) / \Gamma_{0}+\mathrm{i}}+\frac{2 / 3}{2\left(\hbar \omega-\mathrm{E}_{-1 / 2 \rightarrow-1 / 2}\right) / \Gamma_{0}+\mathrm{i}}\right) \\
& \left.+\overrightarrow{\mathbf{P}}_{-1}(\theta)\left(\frac{1}{2\left(\hbar \omega-\mathrm{E}_{-3 / 2 \rightarrow-1 / 2}\right) / \Gamma_{0}+\mathrm{i}}+\frac{1 / 3}{2\left(\hbar \omega-\mathrm{E}_{-1 / 2 \rightarrow 1 / 2}\right) / \Gamma_{0}+\mathrm{i}}\right)\right\}
\end{aligned}
$$

where the subscripts on the energies in the denominator indicate the projection of the spins in the excited and ground states, respectively and $\theta$ is the angle between the quantization axis and the photon wave vector and the polarization matrices are as define in (A.14). The linear basis vectors, are, respectively, parallel and perpendicular to the $\hat{k} \hat{q}$ plane.

Special cases:

\section{Unsplit Line (Isotropic response)}

If the nuclear environment does not include significant fields (e.g. ${ }^{57} \mathrm{Fe}$ in stainless steel) then the energy denominators in (A.11) are all the same and may be factored out. The polarization matrices just sum and the result is independent of the photon propagation direction. One has

$$
\overrightarrow{\mathrm{F}}_{\text {nuc }}(\omega)=-\frac{1}{\mathrm{k}} \frac{\Gamma_{\mathrm{r}}}{\Gamma_{0}} \frac{1}{2 \hbar\left(\omega-\omega_{0}\right) / \Gamma_{0}+\mathrm{i}} \quad \overrightarrow{\mathrm{I}} \quad\left(\begin{array}{l}
\text { Unsplit Line } \\
\text { Dipole Trans. }
\end{array}\right)
$$

where $\overrightarrow{\mathbf{I}}$ is the identity matrix. This is a special case of the general form

$$
\overrightarrow{\mathrm{F}}_{\text {nuc }}(\omega)=-\frac{1}{2 \mathrm{k}} \frac{2 \mathrm{j}_{\mathrm{e}}+1}{2 \mathrm{j}_{\mathrm{g}}+1} \frac{\Gamma_{\mathrm{r}}}{\Gamma_{0}} \frac{1}{2 \hbar\left(\omega-\omega_{0}\right) / \Gamma_{0}+\mathrm{i}} \overrightarrow{\mathbf{I}} \quad \text { (Unsplit Line) (A.17) }
$$


for an unsplit resonance between states with total angular momentum $j_{e}$ in the excited state and $\mathrm{jg}$ in the ground state, (averaged over the spin projections of the ground state).

2. Quantization axis perpendicular to the wave vector.

This is just the case of $\theta=90$ degrees. The polarization matrices all diagonalize so that incident linearly polarized light with polarization $\hat{\mathbf{e}}_{1}$ or $\hat{\mathbf{e}}_{2}$ will be scattered into light of the same polarization. Note that $M= \pm 1$ transitions will couple only to one linear polarization (that parallel to the quantization axis) while the $M=0$ transition will only couple to light with a polarization perpendicular to the quantization direction.

3. Quantization axis parallel to the wave vector.

This is the case for $\theta=0$ or $\theta=180$ degrees. Here the $M=0$ terms drop out of the sum: these transitions will not be excited or scatter. The $M= \pm 1$ polarization matrices then have eigenvectors corresponding to positive and negative helicity light. In particular, setting $\theta=0$ one takes $\hat{\mathbf{q}}=\hat{\mathbf{z}}, \hat{\mathbf{e}}_{1}=\hat{\mathbf{x}}$ and $\hat{\mathbf{e}}_{2}=\hat{\mathbf{y}}$. The eigenvectors for $\overrightarrow{\mathbf{P}}_{1}$ are degenerate and may be written $\hat{\mathbf{e}}_{+}=-\left(\hat{\mathbf{e}}_{1}+\mathbf{i} \hat{\mathbf{e}}_{2}\right) / 2^{1 / 2}$ which corresponds to positive helicity light or left hand circularly polarized. Similarly, the eigenvectors for $\overrightarrow{\mathbf{P}}_{-1}$ are $\hat{\mathbf{e}}_{-}=\left(\hat{\mathbf{e}}_{1}-\mathbf{i} \hat{\mathbf{e}}_{2}\right) / 2^{1 / 2}$ corresponding to the other polarization.

\section{Distribution of Quantization Directions}

Frequently it is necessary to consider a sample containing many nuclei for which there is a distribution of directions for the quantization axes. One example might be an iron foil for which there is no external alignment field (or one that is not sufficiently strong) so that and the domains are not all aligned in the same direction. In this case, we must average over the distribution of quantization 
directions while holding the incident photon direction and polarization fixed. One can not simply integrate equations (A.14), because the polarization directions used to derive the equations depended upon the direction of the incident photon relative to the quantization axis. One must return to A.13, to perform the average. In particular, this average requires evaluation of terms of the form

$$
\overrightarrow{\mathbf{P}}_{M}(\hat{\mathbf{k}})=\int N(\hat{\mathbf{q}}) \overrightarrow{\mathbf{P}}_{M}^{\hat{\mathbf{q}}}(\hat{\mathbf{k}}) \mathrm{d} \boldsymbol{\Omega}_{\hat{\mathbf{q}}}
$$

where $\hat{\mathbf{q}}$ indicates the direction of the quantization axis and $\mathbf{N}(\hat{\mathbf{q}})$ is the distribution.

\section{Special Cases}

1. Uniform Distribution of Quantization Directions.

If there is a uniform distribution of quantization axis directions over all space, then one simply takes $N(\hat{\mathbf{q}})=1 / 4 \pi$ and evaluates the integrals. All dependence on the polarization and direction of photon propagation vector drop out and the matrices reduce to

$$
\overrightarrow{\mathbf{P}}_{1}=\overrightarrow{\mathbf{P}}_{0}=\overrightarrow{\mathbf{P}}_{-1}=\frac{1}{8 \pi} \overrightarrow{\mathbf{I}}
$$

2. Quantization axes uniformly distributed in a plane.

For thin iron samples, there is preferential magnetization within the plane of the material so we consider the case where there may be some distribution of quantization axes within a plane. In particular, in accordance with the work in the main body of this thesis, we concentrate on the case in which the plane containing the distribution of quantization axes includes the direction of photon propagation. One can take $\varphi=0$ and the matrices (A.13) reduce to those in (A.14). These then must be integrated over the appropriate range in $\theta$. Taking the distribution to be uniform over some range $\Delta \theta$ about $\theta_{0}$, one has 


$$
\mathrm{N}(\hat{\mathbf{q}}) \rightarrow\left\{\begin{array}{cc}
\frac{1}{\Delta \theta} & \theta_{0}-\frac{\Delta \theta}{2}<\theta<\theta_{0}+\frac{\Delta \theta}{2} \\
0 & \text { otherwise }
\end{array}\right\}
$$

and $d \Omega_{\hat{\mathrm{q}}} \rightarrow d \theta_{\hat{\mathrm{q}}}$. The polarization matrices become

$$
\begin{aligned}
& \overrightarrow{\mathbf{P}}_{1}=\frac{3}{16 \pi}\left(\begin{array}{cc}
1 & -i \frac{2}{\Delta \theta} \cos \theta_{0} \sin \frac{\Delta \theta}{2} \\
i \frac{2}{\Delta \theta} \cos \theta_{0} \sin \frac{\Delta \theta}{2} & \frac{1}{2}+\frac{1}{2 \Delta \theta} \cos 2 \theta_{0} \sin \Delta \theta
\end{array}\right) \\
& \overrightarrow{\mathbf{P}}_{0}=\frac{3}{8 \pi}\left(\begin{array}{cc}
0 & 0 \\
0 & \frac{1}{2}-\frac{1}{2 \Delta \theta} \cos 2 \theta_{0} \sin \Delta \theta
\end{array}\right)
\end{aligned}
$$

and $\overrightarrow{\mathbf{P}}_{-1}=\overrightarrow{\mathbf{P}}_{1}{ }^{*}$, as always. In particular, we consider three possibilities: uniform distribution in the plane, $\Delta \theta=2 \pi$; distribution about a direction parallel to the propagation direction, $\theta_{0}=0$; and a distribution about a direction perpendicular to the propagation direction, $\theta_{0}=\pi / 2$. In these cases one has

$$
\begin{aligned}
& \overrightarrow{\mathbf{P}}_{1}=\frac{3}{16 \pi}\left(\begin{array}{ll}
1 & 0 \\
0 & \frac{1}{2}
\end{array}\right) \\
& \overrightarrow{\mathbf{P}}_{0}=\frac{3}{8 \pi}\left(\begin{array}{ll}
0 & 0 \\
0 & \frac{1}{2}
\end{array}\right) \\
& \left(\begin{array}{c}
\text { Isotropic Planar } \\
\text { Distribution }
\end{array}\right) \\
& \overrightarrow{\mathrm{P}}_{1}=\frac{3}{16 \pi}\left(\begin{array}{cc}
1 & -\mathrm{i} \frac{2}{\Delta \theta} \sin \frac{\Delta \theta}{2} \\
\mathrm{i} \frac{2}{\Delta \theta} \sin \frac{\Delta \theta}{2} & \frac{1}{2}+\frac{1}{2 \Delta \theta} \sin \Delta \theta
\end{array}\right) \\
& \overrightarrow{\mathbf{P}}_{0}=\frac{3}{8 \pi}\left(\begin{array}{cc}
0 & 0 \\
0 & \frac{1}{2}-\frac{1}{2 \Delta \theta} \sin \Delta \theta
\end{array}\right) \\
& \left(\begin{array}{c}
\text { Planar Distribution } \\
\text { about } \theta_{0}=0
\end{array}\right) \\
& \overrightarrow{\mathbf{P}}_{1}=\frac{3}{16 \pi}\left(\begin{array}{cc}
1 & 0 \\
0 & \frac{1}{2}-\frac{1}{2 \Delta \theta} \sin \Delta \theta
\end{array}\right) \\
& \overrightarrow{\mathbf{P}}_{0}=\frac{3}{8 \pi}\left(\begin{array}{cc}
0 & 0 \\
0 & \frac{1}{2}+\frac{1}{2 \Delta \theta} \sin \Delta \theta
\end{array}\right)
\end{aligned}
$$


One notes that while the eigenpolarizations for the first and third cases are simple linear polarizations, in the second case (planar distribution of quantization axes about the direction of photon propagation) the eigenpolarizations are, in general, elliptical.

\section{References for Appendix A.}

1 H. Frauenfelder, D.E. Nagle, R.D. Taylor, D.R.F. Cochran, and V.M. Visscher, Elliptical Polarization of 57Fe Gamma Rays. Phys. Rev. 126 (1962) 1065.

2 M. Blume and O.C. Kistner, Resonant Absorption in the Presence of a Faraday Rotation. Phys. Rev. 171 (1968) 171.

3 J.P. Hannon and G.T. Trammell, Mössbauer Diffraction. II. Dynamical Theory of Mössbauer Optics. Phys. Rev. 186 (1969) 306.

4 D.E. Brown, Nuclear Dynamical Diffraction Using Synchrotron Radiation, PhD. Thesis, Stanford University, 1993.

5 M. Weissbluth, Atoms and Molecules. San Diego: Academic Press (1978). 


\section{Appendix B: The Distorted Wave Born Approximation}

(DWBA)

The basic assumption in the DWBA is that the scattering system is nearly one for which a complete solution is known: the deviation from the known system is regarded as a perturbation. In the case of rough interfaces, the known solution is that of a flat (ideal) interface (given in chapter 4) and the roughness is the perturbation. In this appendix, the DWBA is evaluated for a graded interface, since work in chapter 5 showed that the effect of a rough interface could be reduced to those of a graded interface, as far as the specular reflectivity is concerned. The approach is somewhat similar to that of Sinha, et al., [Sinha, et al., 1988, 1].

\section{Terms In the DWBA}

The distorted wave Born approximation may be evaluated formally in a manner similar to that described in [Sinha, et al., 1988, 1], [Schiff, 1968, 2]. However, for the case of a graded interface, the appropriate terms are easily included by considering the diagrams in figures B.1 and B.2. The solid heavy horizontal line represents the location for the ideal boundary and the dashed horizontal line is meant to represent a thin layer perturbing the scattering. The solid lines with arrows indicate the solution with an ideal interface while the dashed lines indicates the scattering by the "perturbation" or the deviation from the ideal interface. The perturbation is assumed small so that it only scatters once. Thus, one sees that there are 5 contributions to the reflected wave from the perturbation. The first four are from above the interface, while the last is from below the interface. Figure B.2 shows the diagrams for the contributions to the transmitted wave. 
(1)

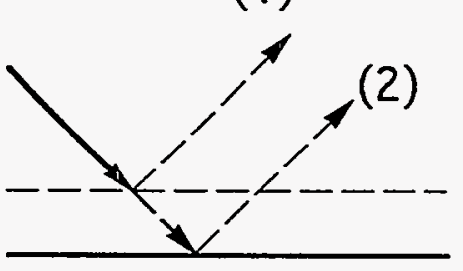

(a)
(3)

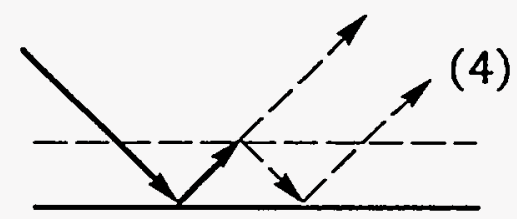

(b)

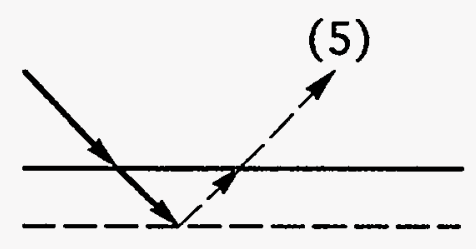

(c)

Figure B.1. Contributions to the reflectivity of a non-ideal interface in the DWBA. The contributions are from scattering by the (a) incident wave, (b) reflected wave and (c) transmitted wave.

(a)

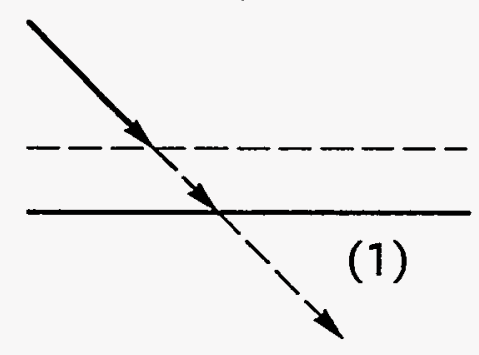

(b)

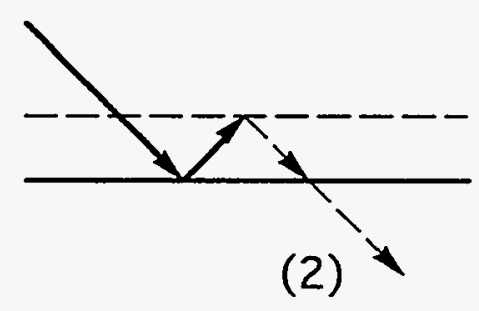

(c)

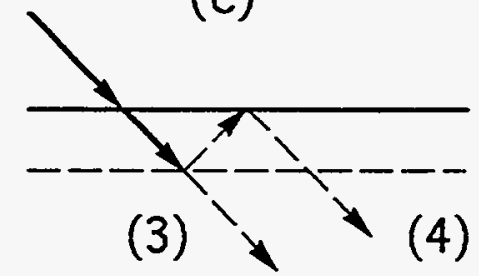

Figure B.2. Contributions to the transmission for a non-ideal interface in the distorted wave Born approximation. The contributions are from scattering by the (a) incident wave, (b) reflected wave and (c) transmitted wave. 
The ideal solution may be taken from the work in chapter 4. In particular, we recall that there were four waves in each materiel, two propagating upward, and two downward (See Fig. 4.1). The form of these waves allows us to write (recall equations (4.29))

$$
\begin{aligned}
& E_{\text {up }}(z)=c_{1} e^{-i k_{1} z} \hat{e}_{1}+c_{2} e^{-i k_{2 p} z} \hat{e}_{2}+c_{3} e^{-i k_{3 p} z} \hat{\mathbf{e}}_{3}+c_{4} e^{-i k_{4} z} \hat{\mathbf{e}}_{4} \\
& E_{d n}(z)=c_{5} e^{-i k_{5} z} \hat{\mathbf{e}}_{5}+c_{6} e^{-i k_{6 p} z} \hat{\mathbf{e}}_{6}+c_{7} e^{-i k_{7 p} z} \hat{e}_{5}+c_{8} e^{-i k_{8} z} \hat{e}_{6}
\end{aligned}
$$

The $z$-dependence has been explicitly included. Using matrices to indicate polarization components, one may write down propagation matrices for each pair of wave fields in the appropriate (homogeneous) layer. Namely,

$$
\begin{aligned}
& E_{\text {inc }}\left(z_{0}+z\right)=\left(\begin{array}{cc}
e^{-i k_{1 p} z} & 0 \\
0 & e^{-i k_{2 p} z}
\end{array}\right) E_{\text {inc }}\left(z_{0}\right) \equiv \overrightarrow{\mathbf{P}}_{1}(z) E_{\text {inc }}\left(z_{0}\right) \\
& E_{\text {ref }}\left(z_{0}+z\right)=\left(\begin{array}{cc}
e^{-i k_{3 p} z} & 0 \\
0 & e^{-i k_{4 p} z}
\end{array}\right) E_{\text {ref }}\left(z_{0}\right) \equiv \overrightarrow{\mathbf{P}}_{1}^{\prime}(z) E_{\text {ref }}\left(z_{0}\right) \\
& E_{\text {trn }}\left(z_{0}+z\right)=\left(\begin{array}{cc}
e^{-i k_{5 p} z} & 0 \\
0 & e^{-i k_{6 p} z}
\end{array}\right) E_{\text {trm }}\left(z_{0}\right) \equiv \overrightarrow{\mathbf{P}}_{2}(z) E_{\text {trn }}\left(z_{0}\right) \\
& E_{\text {inc }}^{\prime}\left(z_{0}+z\right)=\left(\begin{array}{cc}
e^{-i k_{7 p} z} & 0 \\
0 & e^{-i k_{8 p} z}
\end{array}\right) E_{\text {inc }}^{\prime}\left(z_{0}\right) \equiv \overrightarrow{\mathbf{P}}_{2}^{\prime}(z) E_{\text {inc }}^{\prime}\left(z_{0}\right)
\end{aligned}
$$

The second equality in each case defines the propagation matrices.

We take the scattering of a plane perturbation to be represented by a matrix, $\overrightarrow{\mathbf{S}}(z, \phi) d z$, where $\phi$ is the scattering angle and write down the contributions to the reflected and transmitted waves directly from figures (5.3) and (5.4). Care must be taken to properly account for all the phases, which must be referenced to the plane of the ideal interface, $z=0$. In addition, we make use of the fact that the reflection and transmission matrices from the ideal interface problem are known, and we assume (momentarily) that $\mathrm{c}_{7}=\mathrm{c}_{8}=0$. The corrections to the reflectivity and transmission then become 


$$
\begin{aligned}
\Delta \overrightarrow{\mathbf{R}}= & \int_{0}^{+\infty}\left\{\overrightarrow{\mathbf{P}}_{1}^{\prime}(-z) \overrightarrow{\mathbf{S}}(z, 2 \theta) \overrightarrow{\mathbf{P}}_{1}(z)+\overrightarrow{\mathbf{R}} \overrightarrow{\mathbf{P}}_{1}(-z) \overrightarrow{\mathbf{S}}(z, 0) \overrightarrow{\mathbf{P}}_{1}(z)\right\} \mathrm{d} z \\
& +\int_{0}^{+\infty}\left\{\overrightarrow{\mathbf{P}}_{1}^{\prime}(-z) \overrightarrow{\mathbf{S}}(z, 0) \overrightarrow{\mathbf{P}}_{1}^{\prime}(z)+\overrightarrow{\mathbf{R}} \overrightarrow{\mathbf{P}}_{1}(-z) \overrightarrow{\mathbf{S}}(z, 2 \theta) \overrightarrow{\mathbf{P}}_{1}^{\prime}(z)\right\} \mathrm{d} z \mathbf{R} \\
& +\overrightarrow{\mathbf{T}}^{\prime} \int_{-\infty}^{0}\left\{\overrightarrow{\mathbf{P}}_{2}^{\prime}(-z) \overrightarrow{\mathbf{S}}(z, 2 \theta) \overrightarrow{\mathbf{P}}_{2}(z)\right\} \mathrm{d} z \overrightarrow{\mathbf{T}} \\
\Delta \overrightarrow{\mathbf{T}}= & \overrightarrow{\mathbf{T}} \int_{0}^{\infty}\left\{\overrightarrow{\mathbf{P}}_{1}(-z) \overrightarrow{\mathbf{S}}(z, 0) \overrightarrow{\mathbf{P}}_{1}(z)+\overrightarrow{\mathbf{P}}_{1}(-z) \overrightarrow{\mathbf{S}}(z, 2 \theta) \overrightarrow{\mathbf{P}}_{1}^{\prime}(z) \overrightarrow{\mathbf{R}}\right\} \mathrm{d} z \\
& +\int_{-\infty}^{0}\left\{\overrightarrow{\mathbf{P}}_{2}^{\prime}(-z) \overrightarrow{\mathbf{S}}(z, 0) \overrightarrow{\mathbf{P}}_{2}(z)+\overrightarrow{\mathbf{R}}^{\prime} \overrightarrow{\mathbf{P}}_{2}{ }_{2}(-z) \overrightarrow{\mathbf{S}}(z) \overrightarrow{\mathbf{P}}_{2}(z)\right\} \mathrm{d} z \overrightarrow{\mathbf{T}}
\end{aligned}
$$

At this point, it is convenient make several approximations based on the assumption of grazing incidence. In particular, since $\theta$ is small we take

$$
\overrightarrow{\mathbf{S}}(z, 2 \theta) \approx \overrightarrow{\mathbf{S}}(z, 0) \equiv \overrightarrow{\mathbf{S}}(z)
$$

and also assume the solutions to the eigenvalue problem for waves propagating upwards in a material is the same as those for waves propagating downward so that $k_{3 p} \approx-k_{1 p}, k_{4 p} \approx k_{2 p}, k_{7 p} \approx k_{5 p}, k_{8 p} \approx-k_{6 p}$. The propagation matrices become

$$
\begin{aligned}
& \overrightarrow{\mathbf{P}}_{1}^{\prime}(z)=\overrightarrow{\mathbf{P}}_{1}(-z) \\
& \overrightarrow{\mathbf{P}}_{2}^{\prime}(\mathrm{z})=\overrightarrow{\mathbf{P}}_{2}(-z)
\end{aligned}
$$

Re-writing (B.3), one has

$$
\begin{aligned}
\overrightarrow{\mathbf{R}}_{\text {dwba }}=\overrightarrow{\mathbf{R}}+\Delta \overrightarrow{\mathbf{R}=\overrightarrow{\mathbf{R}}+} & \overrightarrow{\mathbf{S}}_{>}(+,+)+\overrightarrow{\mathbf{R}} \overrightarrow{\mathbf{S}}_{>}(-,+)+\overrightarrow{\mathbf{S}}_{>}(+,-) \overrightarrow{\mathbf{R}} \\
& +\overrightarrow{\mathbf{R}} \overrightarrow{\mathbf{S}}_{>}(-,-) \overrightarrow{\mathbf{R}}+\overrightarrow{\mathbf{T}}^{\prime} \overrightarrow{\mathbf{S}}_{<}(+,+) \overrightarrow{\mathbf{T}} \\
\overrightarrow{\mathrm{T}}_{\text {dwba }}=\overrightarrow{\mathrm{T}}+\Delta \overrightarrow{\mathbf{T}}=\overrightarrow{\mathrm{T}}+ & \overrightarrow{\mathrm{T}} \overrightarrow{\mathbf{S}}_{>}(-,+)+\overrightarrow{\mathbf{T}} \overrightarrow{\mathbf{S}}_{>}(-,-) \overrightarrow{\mathbf{R}} \\
& +\overrightarrow{\mathbf{S}}_{<}(-,+) \overrightarrow{\mathbf{T}}+\overrightarrow{\mathbf{R}}^{\prime} \overrightarrow{\mathbf{S}}_{<}(+,+) \overrightarrow{\mathrm{T}}
\end{aligned}
$$

where 


$$
\begin{aligned}
& \overrightarrow{\mathbf{S}}_{>}(\mathrm{a}, \mathrm{b}) \equiv \int_{0}^{\infty} \overrightarrow{\mathrm{P}}_{1}(\mathrm{az}) \overrightarrow{\mathbf{S}}(\mathrm{z}) \overrightarrow{\mathrm{P}}_{1}(\mathrm{bz}) \mathrm{dz} \\
& \overrightarrow{\mathbf{S}}_{<}(\mathrm{a}, \mathrm{b}) \equiv \int_{-\infty}^{0} \overrightarrow{\mathbf{P}}_{2}(\mathrm{az}) \overrightarrow{\mathbf{S}}(\mathrm{z}) \overrightarrow{\mathrm{P}}_{2}(\mathrm{bz}) \mathrm{dz}
\end{aligned}
$$

\section{The Scattering Matrix for a Planar Disturbance}

The form of $\overrightarrow{\mathbf{S}}(z)$ is discussed in chapter 5 . Taking the density of scatterers to be given by (5.23), the scattering amplitude of the perturbation may be given by

$$
\overrightarrow{\tilde{\mathbf{N}}}_{\text {real }}(\mathrm{z})-\overrightarrow{\tilde{\mathbf{N}}}_{\text {ideal }}(\mathrm{z})=\left\{\begin{array}{ll}
\left(\mathrm{N}_{2} \overrightarrow{\mathrm{F}}_{2}-\mathrm{N}_{1} \overrightarrow{\mathrm{F}}_{1}\right) \int_{\mathrm{z}}^{\infty} \mathrm{w}\left(\mathrm{z}^{\prime}\right) \mathrm{d} \mathrm{z}^{\prime} & \mathrm{z}>0 \\
\left(\mathrm{~N}_{1} \overrightarrow{\mathrm{F}}_{1}-\mathrm{N}_{2} \overrightarrow{\mathrm{F}}_{2}\right) \int_{-\infty}^{z} \mathrm{w}\left(\mathrm{z}^{\prime}\right) \mathrm{d} \mathrm{z}^{\prime} & \mathrm{z}<0
\end{array}\right\}
$$

where the polarization effects are preserved in the tensor nature of this quantity. Using (5.16), the form of the scattering matrix is

$$
S_{i j}(z)=\left\{\begin{array}{ll}
\frac{-2 \pi i}{k_{0}} \frac{\hat{\mathbf{e}}_{i}^{*}\left(N_{2} \overrightarrow{\mathbf{F}}_{2}-N_{1} \overrightarrow{\mathbf{F}}_{1}\right) \hat{\mathbf{e}}_{j}}{\sin \theta_{j}} \int_{z}^{\infty} w\left(z^{\prime}\right) d z^{\prime} & z>0 \\
\frac{-2 \pi i}{k_{0}} \frac{\hat{\mathbf{e}}_{i}^{\prime *}\left(N_{1} \overrightarrow{\mathbf{F}}_{1}-N_{2} \overrightarrow{\mathbf{F}}_{2}\right) \hat{\mathbf{e}}_{j}^{\prime}}{\sin \theta_{j}^{\prime}} \int_{-\infty}^{z} w\left(z^{\prime}\right) d z^{\prime} & z<0
\end{array}\right\}
$$

where this assumes $\hat{\mathbf{e}}_{1} \approx \hat{\mathbf{e}}_{3}, \hat{\mathbf{e}}_{2} \approx \hat{\mathbf{e}}_{4}, \hat{\mathbf{e}}_{1}^{\prime} \equiv \hat{\mathbf{e}}_{5} \approx \hat{\mathbf{e}}_{7}, \hat{\mathbf{e}}_{2} \equiv \hat{\mathbf{e}}_{6} \approx \hat{\mathbf{e}}_{8}$ for the purposes of evaluating the product with the scattering amplitude. The angles are those that the respective wave fields make with the planes $\mathrm{z}=$ constant ${ }^{*}$.

\footnotetext{
* This is actually a subtle point. Retuming to (5.16) one sees that it is really the free space wave vector that is being considered. However, the question we are asking here is what is the response of a thin layer imbedded in a dielectric material: one needs to account for both the refraction, the extinction and the absorption of the wave in the dielectric. Thus a better approach is to expand
} 


\section{Simplification for Symmetric Interfaces}

The expressions for the modifications to the reflectivity (B.6, B.7) involve terms of the general forms

$$
\begin{gathered}
F_{>}(q) \equiv i \int_{0}^{\infty} d z e^{i q z} \int_{z}^{\infty} d z^{\prime} w\left(z^{\prime}\right) \\
F_{<}(q) \equiv i \int_{-\infty}^{0} d z e^{i q z} \int_{-\infty}^{z} d z^{\prime} w\left(z^{\prime}\right)
\end{gathered}
$$

In the case that the interface is symmetric about $z=0$, (i.e. $w(z)=w(-z))$ these may be simplified. In particular one has

$$
\mathrm{F}_{>}(\mathrm{q})=\mathrm{F}_{<}(-\mathrm{q})=\frac{1}{2 \mathrm{q}}(w(\mathrm{q})-1)+\mathrm{i} \frac{\mathrm{f}(\mathrm{q})}{\mathrm{q}} \quad\left(\begin{array}{l}
\text { Symmetric } \\
\text { Interface }
\end{array}\right)
$$

The second equality is from noting

$$
\begin{gathered}
\mathrm{i} \int_{0}^{\infty} \mathrm{dz} \mathrm{e}^{\mathrm{i} q \mathrm{q}} \int_{z}^{\infty} \mathrm{d} z^{\prime} \quad w\left(z^{\prime}\right)=\mathrm{i} \int_{0}^{\infty} d z^{\prime} w\left(z^{\prime}\right) \int_{0}^{z^{\prime}} d z e^{\mathrm{i} q z} \\
=\frac{1}{\mathrm{q}} \int_{0}^{\infty} w\left(z^{\prime}\right) \mathrm{e}^{\mathrm{i} q z^{\prime}} d z^{\prime}-\frac{1}{2 \mathrm{q}}
\end{gathered}
$$

and

$$
\begin{aligned}
\int_{0}^{\infty} d z^{\prime} w\left(z^{\prime}\right) e^{i q z^{\prime}} & =\frac{1}{2} \int_{-\infty}^{+\infty} d z^{\prime} w\left(z^{\prime}\right) e^{i q z}+i \int_{0}^{\infty} d z^{\prime} w\left(z^{\prime}\right) \sin \left(q z^{\prime}\right) \\
& \equiv \frac{1}{2} w(q)+i f(q)
\end{aligned}
$$

Equation (B.13) defines $f(q)$. It is useful to note that $w(q)=w(-q)$ for a symmetric interface and $f(q)=-f(-q)$. Finally, expanding $F$ gives

(4.53). One finds that the correct procedure is to replace $k_{0} \sin \theta$ by the appropriate perpendicular component of the wave vector. 


$$
\begin{aligned}
F_{>}(q) & =i \int_{0}^{+\infty} z w(z) d z+q \frac{w^{\prime \prime}(0)}{4}+\Theta\left(q^{2}\right) \\
& =i m_{1}+q \frac{w^{\prime \prime}(0)}{4}+\Theta\left(q^{2}\right)
\end{aligned}
$$

where $\mathrm{m}_{1}$ is the (partial) first moment of $\mathrm{w}(\mathrm{z})$ and $w^{\prime \prime}(0)$ is the second derivative of $w(\mathrm{q})$ evaluated at $\mathrm{q}=0$ (the first derivative is zero for a symmetric interface).

\section{Result of the DWBA}

Using $F_{>}$and $F_{<}(B \cdot 10)$, we can express the components of the matrices $S>$ and $S_{<}$giving

$$
\begin{aligned}
& \mathbf{S}_{>}(\mathrm{a}, \mathrm{b})_{\mathrm{ij}}=-\frac{2 \pi}{\mathrm{k}_{0} \sin \theta_{\mathrm{j}}} \hat{\mathbf{e}}_{\mathrm{i}}^{*}\left(\mathrm{~N}_{2} \overrightarrow{\mathrm{F}}_{2}-\mathrm{N}_{1} \overrightarrow{\mathbf{F}}_{1}\right) \hat{\mathbf{e}}_{\mathrm{j}} \mathrm{F}_{>}\left(-a \mathrm{k}_{\mathrm{pi}}-b \mathrm{k}_{\mathrm{pj}}\right) \\
& \mathbf{S}_{<}(\mathrm{a}, \mathrm{b})_{\mathrm{ij}}=-\frac{2 \pi}{\mathrm{k}_{0} \sin \theta_{\mathrm{j}}^{\prime}} \hat{\mathbf{e}}_{\mathrm{i}}^{\prime^{*}}\left(\mathrm{~N}_{1} \overrightarrow{\mathbf{F}}_{1}-\mathrm{N}_{2} \overrightarrow{\mathbf{F}}_{2}\right) \hat{\mathbf{e}}_{\mathrm{j}}^{\prime} \mathrm{F}_{<}\left(-a \mathrm{k}_{\mathrm{pi}}^{\prime}-b \mathrm{k}_{\mathrm{pj}}^{\prime}\right)
\end{aligned}
$$

where the $1^{\prime}$ is to be associated with the wave field 5 and $2^{\prime}$ with 6 . Thus the corrections to the specular reflection and transmission due to roughness in DWBA have been expressed in terms of known quantities. The equations (B.6), (B.10) and (B.15) express the result for an arbitrary interface profile in a nonisotropic material.

\section{Evaluation for Electronic Scattering}

We evaluate (B.15) for the case of electronic scattering of sigma polarized light. In this case one has

$$
\begin{aligned}
& S_{>}(a, b)=\frac{k_{0}\left(\delta_{2}-\delta_{1}\right)}{\sin \theta} F_{>}\left(-k_{p}(a+b)\right) \\
& S_{<}(a, b)=\frac{k_{0}\left(\delta_{1}-\delta_{2}\right)}{\sin \theta^{\prime}} F_{>}\left(-k_{p}(a+b)\right)
\end{aligned}
$$


so that the corrected reflection and transmission components become

$$
\begin{gathered}
\mathrm{R}_{\mathrm{dwba}}=\mathrm{R}+\frac{\mathrm{k}_{0}\left(\delta_{2}-\delta_{1}\right)}{\sin \theta}\left[\mathrm{F}_{>}\left(-2 \mathrm{k}_{\mathrm{p}}\right)+2 \mathrm{RF}_{>}(0)\right. \\
\left.\quad+\mathrm{R}^{2} \mathrm{~F}_{>}\left(2 \mathrm{k}_{\mathrm{p}}\right)-\frac{\mathrm{k}_{\mathrm{p}}}{\mathrm{k}_{\mathrm{p}}^{\prime}} \mathrm{T}^{\prime} \mathrm{TF} \mathrm{F}_{<}\left(-2 \mathrm{k}_{\mathrm{p}}^{\prime}\right)\right] \\
\mathrm{T}_{\mathrm{dwba}}=\mathrm{T}+\frac{\mathrm{k}_{0}\left(\delta_{2}-\delta_{1}\right)}{\sin \theta}\left[\mathrm{TF}_{>}(0)+\mathrm{RTF}_{>}\left(2 \mathrm{k}_{\mathrm{p}}\right)\right. \\
\left.-\frac{\mathrm{k}_{\mathrm{p}}}{\mathrm{k}_{\mathrm{p}}} \mathrm{TF}_{<}(0)-\frac{\mathrm{k}_{\mathrm{p}}}{\mathrm{k}_{\mathrm{p}}^{\prime}} \mathrm{R}^{\prime} \mathrm{TF}_{<}\left(-2 \mathrm{k}_{\mathrm{p}}^{\prime}\right)\right]
\end{gathered}
$$

Assuming a symmetric interface and putting in the explicit forms for $\mathrm{R}$ and $\mathrm{T}$, these become

$$
\begin{aligned}
& \frac{\mathrm{R}_{\mathrm{dwba}}}{\mathrm{R}}= 1+\frac{\mathrm{k}_{\mathrm{p}}^{\prime}}{2 \mathrm{k}_{\mathrm{p}}}\left(w\left(2 \mathrm{k}_{\mathrm{p}}\right)-1\right)+\frac{\mathrm{k}_{\mathrm{p}}}{2 \mathrm{k}_{\mathrm{p}}^{\prime}}\left(w\left(2 \mathrm{k}_{\mathrm{p}}^{\prime}\right)-1\right) \\
&-\mathrm{i} \frac{\mathrm{k}_{\mathrm{p}}^{2}-\mathrm{k}_{\mathrm{p}}^{\prime 2}}{\mathrm{k}_{\mathrm{p}}} \mathrm{m}_{1}-\mathrm{i} \frac{\mathrm{k}_{\mathrm{p}}^{2}+\mathrm{k}_{\mathrm{p}}^{\prime 2}}{2 \mathrm{k}_{\mathrm{p}}{ }^{2}} \mathrm{f}\left(2 \mathrm{k}_{\mathrm{p}}\right)+\mathrm{i} \frac{\mathrm{k}_{\mathrm{p}}}{\mathrm{k}_{\mathrm{p}}} \mathrm{f}\left(2 \mathrm{k}_{\mathrm{p}}^{\prime}\right) \\
& \frac{\mathrm{T}_{\mathrm{dwba}}}{\mathrm{T}}=1-\left(\mathrm{k}_{\mathrm{p}}-\mathrm{k}_{\mathrm{p}}^{\prime}\right)^{2}\left[\frac{\left(w\left(2 \mathrm{k}_{\mathrm{p}}\right)-1\right)}{8 \mathrm{k}_{\mathrm{p}}{ }^{2}}+\frac{\left(w\left(2 \mathrm{k}_{\mathrm{p}}^{\prime}\right)-1\right)}{8 \mathrm{k}_{\mathrm{p}}^{\prime 2}}\right. \\
&\left.\quad-\mathrm{i} \frac{\mathrm{k}_{\mathrm{p}}+\mathrm{k}_{\mathrm{p}}^{\prime}}{2 \mathrm{k}_{\mathrm{p}} \mathrm{k}_{\mathrm{p}}^{\prime}} \mathrm{m}_{1}+\mathrm{i} \frac{\mathrm{f}\left(2 \mathrm{k}_{\mathrm{p}}\right)}{4 \mathrm{k}_{\mathrm{p}}{ }^{2}}+\mathrm{i} \frac{\mathrm{f}\left(2 \mathrm{k}_{\mathrm{p}}\right)}{4 \mathrm{k}_{\mathrm{p}}^{\prime 2}}\right]
\end{aligned}
$$

Then expanding to lowest order in q one notes that

$$
\begin{aligned}
& w(2 q)-1=2 q^{2} w^{\prime \prime}(0)+\Theta\left(q^{3}\right) \\
& f(2 q)=2 m_{1} q+\Theta\left(q^{3}\right)
\end{aligned}
$$

The corrected reflectivity and transmission then become 


$$
\begin{aligned}
& \frac{\mathrm{R}_{\mathrm{dwba}}}{\mathrm{R}} \approx 1+2 \mathrm{k}_{\mathrm{p}} \mathrm{k}_{\mathrm{p}}^{\prime} w^{\prime \prime}(0) \\
& \frac{\mathrm{T}_{\mathrm{dwba}}}{\mathrm{T}} \approx 1-\frac{1}{2}\left(\mathrm{k}_{\mathrm{p}}-\mathrm{k}_{\mathrm{p}}^{\prime}\right)^{2} w^{\prime \prime}(0)
\end{aligned}
$$

In the event that the distribution is the usual error function, one has $w^{\prime \prime}(0)=-\sigma^{2}$. Equations (B.21) become

$$
\begin{aligned}
\mathrm{R}_{\mathrm{dwba}} & \approx \mathrm{R}\left(1-2 \mathrm{k}_{\mathrm{p}} \mathrm{k}_{\mathrm{p}}^{\prime} \sigma^{2}\right) \\
& \approx \mathrm{R} \mathrm{e}^{-2 \mathrm{k}_{\mathrm{p}} \mathrm{k}_{\mathrm{p}}^{\prime} \sigma^{2}} \equiv \mathrm{R}_{\mathrm{NC}} \\
\mathrm{T}_{\mathrm{dwba}} & \approx \mathrm{T}\left(1+\frac{1}{2}\left(\mathrm{k}_{\mathrm{p}}-\mathrm{k}_{\mathrm{p}}^{\prime}\right)^{2} \sigma^{2}\right) \\
& \approx \mathrm{T} \mathrm{e}^{\left(\mathrm{k}_{\mathrm{p}}-\mathrm{k}_{\mathrm{p}}^{\prime}\right)^{2} \sigma^{2} / 2} \equiv \mathrm{T}_{\mathrm{NC}}
\end{aligned}
$$

where the second approximate equality in each case is only to first order in $\sigma^{2}$.

\section{General Comments}

The expressions (B.22) have been presented previously [Nevot and Croce, 1980, 3], [Sinha, et al., 1988, 1], [Weber and Lengeler, 1992, 4] and Sinha et al gives nice discussion of their applicability. In particular, we note that (B.22a) is really just an approximation to an approximation. However, it has the advantage that it seems to give the correct result in the limit of large angles (the kinematic result, (5.32)), and also agree with the graded result (see calculations in chapter 5) in the limit of small angles, for which it was derived. In fact, the derivation, of the full DWBA (i.e. (B.17)) requires that the incident wave is not greatly perturbed from the Fresnel result for an ideal interface. Thus, as discussed by Sinha, one finds that the full DWBA result is a very poor approximation at larger angles of incidence, while the form given in (B.22) does much better. Thus, on some level, 
the approximation to the DWBA in fact does better than the full DWBA, as a calculational technique to simulate roughness in the case of electronic scattering.

There are no convenient approximations of the form of (B.22) for the more general case of anisotropic media. Here one is left with the more complicated result of the full DWBA. This is probably reasonable at small angles, but should be checked carefully against the result for a graded interface calculation at larger angles. Also, one must be careful when the absorption becomes large (see the discussion in chapter 5.)

\section{References for Appendix B}

1 S.K. Sinha, E.B. Sirota, and S. Garoff, X-ray and neutron scattering from rough surfaces. Phys. Rev. B 38 (1988) 2297.

2 L.I. Schiff, Quantum Mechanics. New York: McGraw-Hill Book Co. (1968).

3 L. Nevot and P. Croce, Caracterisation des surfaces par reflexion rasante de rayons $\mathrm{X}$. Application a l'etude du polissage de quelques verres silicates. Revue Physique. Appliquee 15 (1980) 761.

$4 \mathrm{~W}$. Weber and B. Lengeler, Diffuse Scattering of hard $x$-rays from rough surfaces. Phys. Rev. B 46 (1992) 7953. 